Published in final edited form as:

Birth Defects Res B Dev Reprod Toxicol. 2006 August ; 77(4): 280-397.

\title{
NTP-CERHR Expert Panel Report on the Reproductive and Developmental Toxicity of Soy Formula
}

\author{
Karl K. Rozman ${ }^{1}$, Jatinder Bhatia ${ }^{2}$, Antonia M. Calafat ${ }^{3}$, Christina Chambers ${ }^{4}$, Martine \\ Culty $^{5}$, Ruth A. Etzel ${ }^{6}$, Jodi A. Flaws ${ }^{7}$, Deborah K. Hansen ${ }^{8}$, Patricia B. Hoyer ${ }^{9}$, Elizabeth H. \\ Jeffery ${ }^{10}$, James S. Kesner ${ }^{11, \mp}$, Sue Marty ${ }^{12}$, John A. Thomas ${ }^{13}$, and David Umbach ${ }^{14}$ \\ 1 Department of Pharmacology and Toxicology, University of Kansas Medical Center, Kansas City, KS \\ 2Division of Neonatology, Department of Pediatrics, Medical College of Georgia, Augusta, GA \\ 3National Center for Environmental Health, Centers for Disease Control and Prevention, Atlanta, GA \\ 4Departments of Pediatrics and Family and Preventive Medicine, University of California San Diego Medical \\ Center, San Diego, CA \\ 5Department of Biochemistry and Molecular Biology, Georgetown University Medical Center, Washington, \\ $D C$ \\ 6George Washington University School of Public Health and Health Services, Washington, DC \\ 7Department of Epidemiology and Preventive Medicine, University of Maryland School of Medicine, \\ Baltimore, $M D$ \\ 8Division of Genetic and Reproductive Toxicology, National Center for Toxicological Research, Jefferson, \\ $A R$ \\ 9Department of Physiology, University of Arizona, Tucson, AZ \\ 10Department of Food Science and Human Nutrition, University of Illinois, Urbana, IL \\ 11 National Institute for Occupational Safety and Health, Cincinnati, $\mathrm{OH}$ \\ 12Toxicology Research Laboratory, The Dow Chemical Company, Midland, MI \\ 13Department of Pharmacology and Toxicology, Indiana University School of Medicine, Indianapolis, IN \\ 14National Institute of Environmental Health Sciences, Research Triangle Park, NC
}

\section{PREFACE}

The National Toxicology Program (NTP) and the National Institute of Environmental Health Sciences (NIEHS) established the NTP Center for the Evaluation of Risks to Human Reproduction (CERHR) in June 1998. The purpose of the Center is to provide timely, unbiased, scientifically sound evaluations of human and experimental evidence for adverse effects on reproduction and development caused by agents to which humans may be exposed.

Soy formula was selected for expert panel evaluation because of public concern for the possible health effects. Soy formula contains soy protein isolates and is fed to infants as a supplement

\footnotetext{
Correspondence to: Michael D. Shelby, PhD, NIEHS EC-32, PO Box 12233, Research Triangle Park, NC 27709. E-mail: shelby@niehs.nih.gov.

* Dr. Kesner was unable to participate in the Expert Panel meeting but participated in the drafting and review of the report before and after the meeting.

${ }^{\dagger}$ This article is a U.S. Government work and, as such, is in the public domain in the United States of America.
} 
to or replacement for human milk or cow milk. Soy protein isolates contain phytoestrogens that occur naturally in some legumes, especially soybeans. Phytoestrogens are non-steroidal, estrogenic compounds. In plants, nearly all phytoestrogens are bound to sugar molecules and these phytoestrogen-sugar complexes are not hormonally active. Phytoestrogens are found in many food products in addition to soy formula, especially soy-based foods such as tofu, soy milk, and in some over-the-counter dietary supplements.

To obtain information about soy formula for the CERHR evaluation, the PubMed (Medline) and Toxline databases were searched through February 2006 with genistein and its CAS RN (446-72-0), soy, soya, and relevant keywords. References were also identified from databases such as REPROTOX®, HSDB, IRIS, and DART and from the bibliographies of reports being reviewed.

This evaluation results from the effort of a 14-member panel of government and nongovernment scientists that culminated in a public expert panel meeting held March 15-17, 2006. This report is a product of the expert panel and is intended to (1) interpret the strength of scientific evidence that soy formula is a reproductive or developmental toxicant based on data from in vitro, animal, or human studies, (2) assess the extent of human exposures to include the general public, occupational groups, and other sub-populations, (3) provide objective and scientifically thorough assessments of the scientific evidence that adverse reproductive/ developmental health effects may be associated with such exposures, and (4) identify knowledge gaps to help establish research and testing priorities to reduce uncertainties and increase confidence in future evaluations. This report has been reviewed by members of the expert panel and by CERHR staff scientists. Copies have been provided to the CERHR Core Committee that is made up of representatives of NTP-participating agencies.

This Expert Panel Report will be included in the subsequent NTP-CERHR Monograph on the Potential Human Reproductive and Developmental Effects of Soy Formula. This monograph will include the NTP-CERHR Brief, the Expert Panel Report, and all public comments on the Expert Panel Report. The NTP-CERHR Monograph will be made publicly available and transmitted to appropriate health and regulatory agencies.

The NTP-CERHR is headquartered at NIEHS, Research Triangle Park, NC and is staffed and administered by scientists and support personnel at NIEHS and at Sciences International, Inc., Alexandria, Virginia.

\subsection{CHEMISTRY, USE, AND HUMAN EXPOSURE}

\subsection{Chemistry}

Soy infant formula refers to infant food made using soy protein isolate and other components. The term, "soy formula" is used as a synonym for "soy infant formula" in this report. The terms "soy" and "soybean" are used commonly for the leguminous Asian plant Glycine max. Soybean is also used to designate the edible seed of this plant. In this report, the term "soy" is used as an adjective to denote products derived from the edible seed (e.g., soy milk, soy formula, soy meal) and "soybean" is used to refer to the edible seed itself. In the U.S., the nutrient composition of soy and other infant formulas is regulated by the Food and Drug Administration (FDA, 2000). Table 1 lists the primary ingredients in some common brands of powdered soy infant formulas. Those ingredients include corn syrup, soy protein isolate, vegetable oils, and sugar (Drugstore.com, 2004). In addition, the formulas are fortified with nutrients such as iron, calcium, phosphorous, magnesium, zinc, manganese, copper, iodine, selenium sodium, potassium, chloride, choline, inositol, and vitamins A, C, D, E, K, and B (B1, B2, B6, B12, niacin, folic acid, pantothenic acid, biotin). Before feeding, 8.7-9.3 g powdered formula is 
added to 2 fluid ounces of water (Drugstore.com, 2004). Soy formulas are also available as concentrated liquids and as ready-to-feed formulations (USDA, 2002b).

Contaminants of soy protein include phytates (1.5\%), which bind minerals, and protease inhibitors, which have antitrypsin, antichymotrypsin, and anti elastin properties (AAP, 1998). As discussed further in Section 1.2.1, formulas are fortified with minerals to compensate for phytate binding and heated to inactivate protease inhibitors. Aluminum is found in soy formulas at concentrations of $600-1300 \mathrm{ng} / \mathrm{mL}$, levels that exceed aluminum concentrations in human milk, 4-65 ng/mL (AAP, 1998). Mineral salts added to soy formulas are the source of aluminum.

Soy products contain phytoestrogens of the isoflavone class (MAFF, 1998b; Setchell et al., 1998; UK Committee on Toxicity, 2003). Isoflavones can be conjugated to glucose or other carbohydrate moieties. Carbohydrate conjugates are generically called glycosides, and glucose conjugates are called glucosides. Small amounts of isoflavones are present in soy products in their unconjugated (aglycone) forms. The three main aglycones found in soy formula are genistein, daidzein, and to a smaller extent, glycitein. The genistein content of soy formula has been the subject of commentary because of concern about exposure of infants to estrogenic compounds; however, soy formula and other soy-based foods contain many components of which genistein is only one. In this report, attention will be focused on the effects of feeding soy formulas and other soy-based products to humans or experimental animals. A separate CERHR Expert Panel Report on Genistein (Rozman et al., in press) will focus on the effects of administering genistein itself, isolated from other plant components. When studies have been carried out using administration of isoflavone mixtures, the Expert Panel will judge whether the isoflavone mixture is expected to be informative on possible effects of feeding soy products or the mixture is more appropriately discussed as estimating possible effects of genistein. The Expert Panel recognizes that it is not entirely satisfactory to discuss isoflavone mixtures as either representing soy foods or purified genistein.

Chen and Rogan (2004) reported that only 3.2-5.8\% of total isoflavone in soy formula consists of unconjugated genistein and daidzein, and that amounts can vary by batch. The majority $(<65 \%)$ of isoflavones detected in soy formula are conjugated to sugar molecules to form glycosides (Setchell et al., 1998). Glucose groups on glycoside compounds can be esterified with acetyl or malonyl groups to form acetyl- or malonylglycosides (UK Committee on Toxicity, 2003). Isoflavones detected in soy infant formula by Setchell et al. (Setchell et al., 1998) or the U.K. Ministry of Agriculture, Fisheries, and Food (MAFF, 1998b) are listed in Figure 1, along with their structures.

Conjugation with glucose groups increases water solubility of isoflavones, which are low molecular-weight hydrophobic compounds (UK Committee on Toxicity, 2003). Glucoside compounds can be deconjugated to form the biologically active aglycone compound by gut microflora (MAFF, 1998b), under acidic conditions (UK Committee on Toxicity, 2003), or by metabolic enzymes (Setchell et al., 1998). Therefore, exposure to a particular isoflavone (e.g., genistein) is theoretically the sum of the aglycone and respective glycoside compound concentrations converted on the basis of molecular weight. However, the aglycone is reconjugated in the gut wall leaving approximately 1-2\% free aglycone to enter the portal circulation. Chen and Rogan (2004) report that isoflavones are glucuronidated and circulated primarily in conjugated form. Estimated infant exposures to particular isoflavones through soy formula are discussed in Section 1.2.3.

Setchell et al. (1998) stated that isoflavone levels in soybeans can vary as a result of geographic location, climate, and growing conditions. They noted that high temperatures during processing of formula could affect the profile of conjugated isoflavones but are not expected to affect total 
isoflavone levels. For example, malonyl groups are especially heat labile and decompose to form their respective acetylglycoside compounds.

\subsection{Use and Human Exposure}

1.2.1 Production information-In the manufacture of soy infant formula, the hull of the soybean is removed and the pulp is processed into oil and flake (AAP, 1998). Soy protein isolate is extracted from the flake using a slightly alkaline solution and is precipitated at the isoelectric point of 4.5 . The resulting isolate has a purity of $\geq 90 \%$ soy protein. The soy protein isolate is fortified with L-methionine, L-carnitine, and taurine. L-methionine improves the biological quality of the protein. Carnitine is needed for oxidation of long-chain fatty acids. Taurine is an antioxidant that is a major conjugate of bile acids in infants. Both carnitine and taurine are added at concentrations found in human milk. Vegetable oils such as soy, palm, sunflower, olein, safflower, and coconut are added to provide fats. Corn starch, tapioca starch, and sucrose are used as carbohydrate sources. Phytates, which bind minerals, are present in soy proteins at $1.5 \%$. Therefore, total phosphorus and calcium are added at concentrations that are $20 \%$ higher than in cow-milk formulas, and the formulas are supplemented with iron and zinc. Heat applied during the processing of soy protein removes $80-90 \%$ of protease inhibitor activity.

Manufacturers of soy formula and some brand names of soy formula sold in the U.S. are listed in Table 2.

1.2.2 Use-Soy formulas are used generally to feed infants who are allergic to dairy products or are intolerant of lactose, galactose, or cow-milk protein (Essex, 1996; Tuohy, 2003). Infants are often changed from cow-milk to soy formula when they have symptoms such as colic, crying, diarrhea, and vomiting (Forsyth et al., 1985). Some parents feed their infants soy formula to maintain a vegetarian lifestyle or because of perceived health benefits of soy food consumption (Badger et al., 2002). Soy formula is not recommended currently for preterm infants.

Essex (1996) reported that soy formulas represented 7\% of infant formula sales in the U.K., $13 \%$ in New Zealand, and 10-20\% in the U.S. A 1998 infant-feeding survey conducted by Ross Products Division indicated that $18 \%$ of infants are fed soy formula during the first year of life (Strom et al., 2001). Based on the 2000 U.S. Census estimate of 4 million American infants younger than 1 year of age, Strom et al. (2001) estimated that 750,000 U.S. infants per year are fed soy formula. According to marketing data and hospital discharge records, it has been estimated that $25 \%$ of newborns in the U.S. are fed soy formula (Badger et al., 2002). A study conducted at Yale University examined formula changes in 189 breast-fed infants and 184 formula-fed infants and reported that a total of 87 infants [23\%] received soy formula sometime during the first 4 months of life (Forsyth et al., 1985). A telephone survey in Israel identified soy formula feeding in $31.5 \%$ of 1803 infants at some time during the first year of life (Berger-Achituv et al., 2005). Of the children on soy formula, $65 \%$ used it for 12 months or more. The decision to use soy formula as opposed to cow-milk formula was made by the mother rather than a health care provider in the majority of instances in the Israeli survey. The mother's decision was most often based on her personal preference rather than concerns for cow milk allergies or for symptoms.

1.2.3 Occurrence and infant exposure-Levels of isoflavones and their glycosides in infant soy formula were reported in a survey of soy foods conducted by the U.S. Department of Agriculture (USDA) and Iowa State University (USDA, 2002b). The survey included results published by Murphy et al. (1997), Nguyenle et al. (1995), and Setchell et al. (1997).

Unpublished data and analyses conducted at Iowa State University were also included in the 
survey. Results were presented for the most common isoflavones, genistein, daidzein, and glycitein, and their glycosides, although some studies did not include glycitein values. Glycoside values were converted to free-form (aglycone) values. Total isoflavones were calculated if values were available for daidzein and genistein equivalents, but it was noted that reported total isoflavone values may not equal values obtained by adding individual isoflavone equivalents. The survey included results for formula powders, liquid concentrates, and reconstituted or ready-to-eat formulas. Results of the infant soy formula survey are reported in Table 3. Reconstituted or ready-to-feed soy formulas contained genistein equivalents 1.58 $2.26 \mathrm{mg} / 100 \mathrm{~g}$ formula [15.8-22.6 $\boldsymbol{\mu g} / \mathbf{g}$ formula], daidzein equivalents $0.75-1.91 \mathrm{mg} / 100 \mathrm{~g}$ formula [7.5-19.1 $\boldsymbol{\mu g} / \mathbf{g}$ formula], glycitein equivalents $0.28-0.35 \mathrm{mg} / 100 \mathrm{~g}$ formula [2.8-3.5 $\boldsymbol{\mu g} / \mathbf{g}$ formula], and total isoflavone equivalents $2.63-4.17 \mathrm{mg} / 100 \mathrm{~g}$ formula [26.3-41.7 $\boldsymbol{\mu} / \mathbf{g}$ formula]. As expected, levels of isoflavone equivalents were higher in soy formula powders and liquid concentrates. Percentages for individual isoflavones were genistein equivalents $36.8-70.1 \%$, daidzein equivalents $18.2-45.8 \%$, and glycitein equivalents $7.9-13.0 \%$.

In studies supported by Wyeth Laboratories, Protein Technologies International, and the National Institutes of Health (NIH), Setchell et al. (1997) measured isoflavone levels in infant soy formula and in the blood of infants $(n=7)$ consuming soy formula. Results of the study, as well as additional details about methodology, were published in a later report by Setchell et al. (1998). After extraction with methanol, isoflavone levels in five U.S. infant formulas were measured by high performance liquid chromatography (HPLC). Genistein and daidzein conjugates, mainly glycosides, were the most abundant isoflavone-related compounds identified. Mean percentages of isoflavones and their respective conjugates were reported at $67.1 \%$ genistein equivalents, $28.7 \%$ daidzein equivalents, and $~ 5 \%$ glycitein equivalents in soy infant formula. Levels of individual isoflavones, based on conversion to aglycone concentrations, are included in Table 3, which summarizes the USDA survey of soy formulas. Total average isoflavone equivalents concentrations were reported at $32-47 \mathrm{mg} / \mathrm{L}$ in prepared formulas. Table 4 lists estimated intakes of isoflavones and the assumptions used to estimate exposure. Depending on infant age, isoflavone equivalents intake was estimated at 6-12 mg/ $\mathrm{kg}$ bw/day. A comparison of estimated isoflavone equivalents intake from soy formulas available in different countries is included in Table 5, which also includes estimates of individual isoflavone equivalents levels based on mean levels detected in formula.

Franke et al. (1998) measured isoflavone+conjugate levels in four U.S. brands of soy formula using methanol extraction and HPLC with diode-array ultraviolet detection. Isoflavone conjugation patterns were similar to those found in soy foods and included malonates (32$43 \%$ ), glucosides (37-52\%), acetates (6-7\%), and aglycones (9-13\%). Total isoflavones were measured at $155.1-281.4 \mu \mathrm{g} / \mathrm{g}$. Percentages of each type of isoflavone included $55.3-57.7 \%$ genistein equivalents, 34-36.1\% daidzein equivalents, and 7.4-8.5\% glycitein equivalents. Based on an intake of $1 \mathrm{~L}$ formula, a body weight of $4.5 \mathrm{~kg}$, and instructions for preparing formula, the authors estimated infant isoflavone equivalents exposure at $\sim 7 \mathrm{mg} / \mathrm{kg}$ bw/day. The authors stated that isoflavone+conjugate exposure in infants fed soy formula is 4-6 times higher than in adults eating a soy-rich diet ( $30 \mathrm{~g} /$ day $)$.

Irvine et al. (1998a) used an HPLC technique to measure isoflavone equivalents levels in infant soy formulas and foods such as cereals and pureed meats or vegetables purchased in New Zealand. In both soy formula and infant foods, the majority of genistein and daidzein were present as their respective glucosides. In soy formula, total concentrations of isoflavones (mean \pm SEM) were $87 \pm 3 \mu \mathrm{g} / \mathrm{g}$ genistein and $49 \pm 2 \mu \mathrm{g} / \mathrm{g}$ daidzein. [If it is assumed that genistein and daidzein are the only isoflavones in the formulas, the percentages of total isoflavone represented by each compound are $64 \%$ genistein equivalents and $36 \%$ daidzein equivalents.] Estimated infant exposures resulting from soy formula intake are summarized in Table 6. In three different infant cereals [composition not indicated] and two different 
infant dinners [composition not indicated], genistein+conjugate levels were measured at 3$287 \mathrm{mg}$ aglycone equivalents/kg food and daidzein levels at 2-276 mg aglycone equivalents/ $\mathrm{kg}$ food. The study authors noted that a single serving of one of the cereals could result in isoflavone exposures ranging from $0.01-0.8 \mathrm{mg}$ aglycone equivalents $/ \mathrm{kg}$ bw/day in a 4-monthold infant, thus increasing isoflavone intake by $>25 \%$.

Murphy et al. (1997) analyzed six brands of soy formula sold in the U.S. and reported that total isoflavone levels were similar across brands and were comprised of 59\% genistein equivalents, $29 \%$ daidzein equivalents, and $12 \%$ glycitein equivalents. Levels of isoflavones and their conjugates are reported in the USDA survey (Table 3), and estimates of infant exposure are reported in Table 6.

Hoey et al. (2004) used a liquid chromatography-mass spectrometry (LC-MS) method to measure isoflavone+conjugate levels in three soy-based infant formulas from the U.K. The total isoflavone content of soy formulas consisted of 58-67\% genistein equivalents, $27-29 \%$ daidzein equivalents, and 6-16\% glycitein equivalents. The study authors estimated that 4-6month-old infants consumed between 17.5 and $33.0 \mathrm{mg} /$ day isoflavone equivalents or 1.7-4.4 $\mathrm{mg}$ aglycone equivalents $/ \mathrm{kg} \mathrm{bw} /$ day. Infants fed the cow-milk formula with the highest isoflavone content $(2.1 \mathrm{mg} / \mathrm{kg}$ formula) were estimated to consume $0.16-0.27 \mathrm{mg} /$ day isoflavone equivalents, or $0.02-0.03 \mathrm{mg}$ aglycone equivalents $/ \mathrm{kg}$ bw/day.

The U.K. Ministry of Agriculture, Fisheries, and Food (MAFF, 1998a,b) conducted a survey of isoflavone levels in 6 brands of infant soy formulas. The formulas were analyzed for 14 different isoflavones and their conjugates and one coumestan compound using methanol extraction followed by HPLC and LC-MS. Isoflavone levels were normalized to aglycone concentrations. Isoflavones were detected in all soy formulas at concentrations of $18-41 \mathrm{mg} /$ $\mathrm{L}$ made-up formula. The majority of iso-flavones were present as glycosides, but smaller amounts of acetyl and malonyl forms and aglycones were also present. The most abundant isoflavones, genistein-, daidzein-, and glycitein-related compounds, represented an average of 58,36 , and $6 \%$ of formulations on a molar basis, respectively. The isoflavones/conjugates glycitein, 6'-O-acetylglycitin, formononetin, and biochanin A and the coumestan coumestrol were not detected. Eight batches of one soy formula, SMA Wysoy, purchased at different times and locations were analyzed to determine variation between batches. Isoflavone concentrations were $18-33 \mathrm{mg}$ aglycone equivalents/L formula as made up. The U.K. Ministry of Agriculture, Fisheries, and Food concluded that isoflavone levels did not vary enough to significantly impact exposure. Statistical analyses by analysis of variance (ANOVA) and $F$-test did not find a significant difference at the 5\% level in isoflavone levels between batches of the same brand and between different brands.

The U.K. Ministry of Agriculture, Fisheries, and Food summarized and compared levels of isoflavones and their conjugates measured in soy formula from other countries, and those values are outlined in Table 5. The Ministry of Agriculture, Fisheries, and Food concluded that isoflavone levels in soy formula from the U.K. were similar to concentrations reported in other countries; differences most likely resulted from batch variations in soy isolate isoflavone levels, slight variations in formulas, and minor differences in analytic methodology.

The U.K. Ministry of Agriculture, Fisheries, and Food estimated isoflavone+conjugate intake in 1-2-month-old and 4-6-month-old infants based on survey results, average body weight, and intake data. Isoflavone+conjugate intake was estimated at $5 \mathrm{mg}$ aglycone equivalents $/ \mathrm{kg}$ bw/day in 1-2-month-old infants and $4.5 \mathrm{mg}$ aglycone equivalents/kg bw/day in 4-6-monthold infants. A comparison of the U.K. values with values obtained from other countries is included in Table 6. 
Setchell et al. (1997, 1998) used a gas chromatography (GC)-MS method to compare isoflavone levels in soy formula and human milk and to measure isoflavone levels in human milk after soy food ingestion. In comparison to soy formula, levels of isoflavones (measured by GC/MS) were substantially lower in cow-milk formula (below detection limit, which was not reported) and in milk collected from nine healthy, omnivorous, lactating women (mean $\pm \mathrm{SD}=5.6 \pm 4.4$ $\mu \mathrm{g} / \mathrm{L})$ (Setchell et al., 1998). Equol was detected in seven of nine human-milk samples. A >10fold increase in milk isoflavone levels was observed after ingestion of $30 \mathrm{mg}$ isoflavones + conjugates in the form of $10 \mathrm{~g}$ toasted soy nuts. [Figure 2 in the study report suggests that milk daidzein levels increased $>40$-fold and genistein levels increased $>10$-fold 1 day after the ingestion of soy nuts.] The study authors noted that isoflavones occur predominantly as glucuronide conjugates in human milk. The U.K. Ministry of Agriculture, Fisheries, and Food (MAFF, 1998a) analyzed three cow-milk formulas for isoflavones using HPLC and LC-MS methods, and reported that isoflavone levels were below the detection limit of $0.25-0.5 \mathrm{mg} / \mathrm{L}$. Irvine et al. (1998a) used an HPLC method to measure isoflavone levels in dairy-based formula and in human milk. Levels of genistein and daidzein were below the detection limit $(0.1 \mu \mathrm{g} /$ $\mathrm{g}$ ) in human milk samples from 11 mothers and in the dairy-based formulas. Hoey et al. (2004) used an LC-MS method to measure isoflavone levels in cow milk-based infant formulas from the UK. Isoflavones were not detected in the majority of the cow-milk infant formulas (0.5 mg/kg detection limit). King et al. (1998) conducted HPLC analyses on Australian cow milk and reported mean isoflavone levels of $<5 \mathrm{ng} / \mathrm{mL}$ daidzein, 4-29 ng/mL genistein, and 45-293 ng/mL equol.

After hydrolyzing samples to convert conjugated isoflavones to aglycones, Setchell et al. (1997, 1998) used a GC-MS method to measure total plasma isoflavone levels in seven 4month-old male infants fed Isomil soy formula. Mean \pm SD levels of genistein and daidzein were reported at $683 \pm 442.6$ and $295.3 \pm 59.9 \mu \mathrm{g} / \mathrm{L}$, respectively, and total isoflavones were reported at 552-1775 $\mu \mathrm{g} / \mathrm{L}$ (mean $980 \mu \mathrm{g} / \mathrm{L}$ ). [Plasma glycitein levels were not measured.] The study authors noted that they did not attempt to measure the extent of isoflavone conjugation in infant plasma. These total plasma isoflavone levels were 50-100-fold higher in infants fed soy formula compared to 4-month-old male infants fed human milk (mean \pm SD 4.7 $\pm 1.3 \mu \mathrm{g} / \mathrm{L}, \mathrm{n}=7$ ) and cow-milk formula (mean \pm SD 9.3 $\pm 1.2 \mu \mathrm{g} / \mathrm{L}, \mathrm{n}=7$ ). Plasma isoflavone levels in infants fed soy formula were also higher compared to adults (50-200 $\mu \mathrm{g} / \mathrm{L})$ and Japanese adults (40-240 $\mu \mathrm{g} / \mathrm{L})$ ingesting similar levels of isoflavones+conjugates from soybased foods.

The CERHR Expert Panel Report on Genistein provides estimates of adult exposures to isoflavones + conjugates and allows for a comparison of isoflavone exposures in infants fed soy formula (Rozman et al., in press). Most estimates of adult exposure were based on dietary surveys, whereas infant exposures were based on isoflavone levels measured in soy formula and assumed body weight and formula intake. Based on limited information provided in the Genistein report, mean or median genistein + conjugate exposures are estimated at $\leq 1 \mathrm{mg}$ aglycone equivalents/day (0.01 mg aglycone equivalents/kg bw/day assuming a $70 \mathrm{~kg} \mathrm{bw})$ in U.S. adults with no specified dietary preferences, $10-15 \mathrm{mg}$ aglycone equivalents/day (0.1$0.2 \mathrm{mg}$ aglycone equivalents/kg bw/day) in semi-vegetarian or vegetarians in one U.S. survey, and 5-50 mg aglycone equivalents/day (0.1-1 mg aglycone equivalents/kg bw/day) in Asian adults. Based on mean $\mathrm{mg} / \mathrm{kg}$ bw/day intake estimates, infants fed soy formula can be exposed to isoflavone+conjugate levels that are two to three orders of magnitude higher than U.S. adults with low isoflavone exposure, one to two orders of magnitude higher than vegetarians in Western countries, and within the same order of magnitude to one order of magnitude higher than Asians. Comparisons of isoflavone+conjugate intake in infants and adults based on food or formula intake were fairly consistent with comparisons based on blood isoflavone levels presented in Section 2. 
Differences in soy food exposure patterns throughout life were noted for Americans compared to Asians (Badger et al., 2002). In the U.S., typical diets are low in soy food intake, and the fetus is thus exposed to low levels of genistein. Significant exposure to genistein and its conjugates occurs in the approximately $25 \%$ of infants who are fed soy formula. After those infants are weaned, soy food intake and genistein exposure drops and typically remain low over the lifetime. In Asian cultures consuming soy products, the fetus is exposed to genistein and its conjugates as a result of maternal soy food intake. At birth, most infants are either breast fed or fed cow-milk formula, so exposure to genistein is very low during infancy. On weaning, the infants begin receiving soy foods and exposure to soy products and genistein+conjugates remain high over their lifetime.

\subsection{Utility of Data}

The human exposure data consist of a database reporting isoflavone+conjugate levels in soy formulas in the U.S. and other countries. Exposures of infants to isoflavones and their conjugates through consumption of soy formulas have been estimated based on levels of isoflavones+conjugates measured in formulas, formula intakes, and infant body weights. One study reported blood levels of isoflavones in infants fed soy formulas and compared the values to infants fed cow milk or human milk and to adults who ingested soy products. The available data provide a good foundation for estimating approximate exposure and dose within broad populations or within individuals when the soy formula and the infants' weight and age are known.

\subsection{Summary of Human Exposure Data}

Soy formula refers to infant food made using soy protein isolate and other components such as corn syrup, vegetable oils, and sugar (Drugstore.com, 2004). The soy protein isolate is fortified with L-methionine, L-carnitine, and taurine (AAP, 1998). The formulas are fortified with nutrients (Drugstore.com, 2004). Contaminants of soy protein include phytates (1.5\%), which bind minerals, and protease inhibitors, which have antitrypsin, antichymotrypsin, and anti elastin properties (AAP, 1998). Phosphorus, calcium, iron, and zinc are added to soy formula to compensate for phytate binding of minerals. Heat applied during the processing of soy protein removes $80-90 \%$ of protease inhibitor activity. Aluminum is present in soy formulas because of the addition of mineral salts.

Soy products contain phytoestrogens of the isoflavone class (MAFF, 1998b; Setchell et al., 1998; UK Committee on Toxicity, 2003). The primary isoflavones detected in soybeans are derived from genistein, daidzein, and to a smaller extent, glycitein. The majority $(>65 \%)$ of isoflavones in soy formula are conjugated to sugar molecules to form glycosides (Setchell et al., 1998). Glucose groups in glycoside compounds can be esterified with acetyl or malonyl groups to form acetyl- or malonyl glycosides (UK Committee on Toxicity, 2003). Small amounts of genistein and daidzein $(3.2-5.8 \%)$ are present in soy products in their unconjugated (aglycone) forms (Chen and Rogan, 2004). Isoflavones detected in soy infant formula by Setchell et al. (1998) or the U.K. Ministry of Agriculture, Fisheries, and Food (MAFF, 1998b) are listed in Figure 1 with their structures. Because glycosidic compounds can be deconjugated in the body to form the biologically active aglycone compound, exposure to a particular isoflavone (e.g., genistein) is theoretically the sum of the aglycone and respective glycoside compound concentrations converted on the basis of molecular weight (MAFF, 1998b; Setchell et al., 1998; UK Committee on Toxicity, 2003). Isoflavone levels in soybeans are reported to vary as a result of geographic location, climate, and growing conditions (Setchell et al., 1998).

It is estimated that $10-20 \%$ of infants in the U.S. are fed soy formula some time during the first year of life (Essex, 1996; Strom et al., 2001). Soy formulas are generally used to feed 
infants who are allergic to dairy products or are intolerant of lactose, galactose, or cow-milk protein (Essex, 1996; Tuohy, 2003). Infants are sometimes given soy formula when they have symptoms such as colic, crying, diarrhea, or vomiting (Forsyth et al., 1985) or to maintain a vegetarian or perceived healthy lifestyle (Badger et al., 2002).

A number of studies measured total isoflavone (aglycone + glycoside) levels in infant formulas, determined percentages of individual isoflavones, or estimated infant exposures. In a USDA survey of infant formulas, total isoflavone levels reported in reconstituted formulas or readyto-feed formulas were reported at 15.8-22.6 $\mu \mathrm{g}$ genistein equivalents/g formula, 7.5-19.1 $\mu \mathrm{g}$ daidzein equivalents/g formula, 2.8-3.5 $\mu \mathrm{g}$ glycitein equivalents/g formula, and 26.3-41.7 $\mu \mathrm{g}$ total isoflavone equivalents/g formula (USDA, 2002b). On a mg isoflavone equivalents/L ready-to-feed formula basis, total isoflavone levels were reported at $25-47 \mathrm{mg} / \mathrm{L}$ in U.S. soy formulas (Murphy et al., 1997; Setchell et al., 1998) except for one study reporting a total isoflavone level of $155-281 \mathrm{mg} / \mathrm{L}$ (Franke et al., 1998) and at $18-41 \mathrm{mg} / \mathrm{L}$ in formulas obtained from the U.K. and Australia (Knight et al., 1998; MAFF, 1998a,b). Percentages of individual isoflavones representing total isoflavone levels in U.S. soy formulas were estimated at 36.8$70.1 \%$ genistein equivalents, $18.2-45.8 \%$ daidzein equivalents, and 5-13.0\% glycitein equivalents (Murphy et al., 1997; Setchell et al., 1997, 1998; Franke et al., 1998; USDA, $2002 b$ ). Percentages of individual isoflavones in formulas from the U.K. were reported at 58$67 \%$ genistein equivalents, $27-36 \%$ daidzein equivalents, and 6-16\% glycitein equivalents (MAFF, 1998a,b; Hoey et al., 2004).

Isoflavone exposure through soy formula intake has been estimated in the U.S. and other countries based on total isoflavone levels measured in soy formulas and assumptions of formula intakes and infant body weights. In the U.S., total isoflavone intake by infants was estimated at 1-12 mg/kg bw/day (Murphy et al., 1997; Setchell et al., 1997, 1998; Franke et al., 1998). In the U.K. and New Zealand, isoflavone intake by infants was estimated at $1.7-5 \mathrm{mg} / \mathrm{kg} \mathrm{bw} /$ day (Irvine et al., 1998a; MAFF, 1998a,b; Hoey et al., 2004).

Mean \pm SD plasma levels of total isoflavones in infants fed soy formula were reported at 683 $\pm 442.6 \mu \mathrm{g} / \mathrm{L}$ for genistein equivalents, $295.3 \pm 59.9 \mu \mathrm{g} / \mathrm{L}$ for daidzein equivalents, and $552-$ $1775 \mu \mathrm{g} / \mathrm{L}$ (mean $980 \mu \mathrm{g} / \mathrm{L}$ ) for total isoflavones (Setchell et al., 1997, 1998). Mean plasma total isoflavone levels were $\sim 2$ orders of magnitude higher than in infants fed cow-milk formula or human milk. Mean plasma levels of infants fed soy formulas were 5-20 times higher than Japanese adults or adults ingesting similar levels of total isoflavones from soy-based foods, 50 times higher than vegetarian adults in Western populations, and 500 times higher than omnivorous adults in Western populations (Setchell et al., 1997, 1998) (Table 7). Dietary exposure estimates comparing total isoflavone intake in infants ingesting soy formula versus adults are fairly consistent to comparisons based on plasma total isoflavone levels [see (Rozman et al., in press)].

\subsection{GENERAL TOXICOLOGY AND BIOLOGICAL EFFECTS}

\subsection{Toxicokinetics and Metabolism}

This section focuses on toxicokinetics and metabolism of 2 of the isoflavones, genistein and daidzein, found in soy formula and other soy foods. There are few studies available on toxicokinetics in infants. Therefore, a large part of this section is based on studies conducted in adults. Studies that focus on toxicokinetics after exposure to pure genistein are discussed in the CERHR Expert Panel Report on Genistein (Rozman et al., in press).

\subsubsection{Human}

2.1.1.1 Absorption: Genistein and daidzein exist mainly as glycosides in unfermented soy foods. Due to their high water solubility and molecular weight, isoflavone glycosides are not 
readily absorbed across the gastrointestinal tract, as shown in a study of adults by Setchell et al. (1998) (reviewed in UK Committee on Toxicity, 2003). For absorption to occur, isoflavones must first be hydrolyzed to their aglycones, which have some hydrophobicity and lower molecular weights. Absorption occurs primarily in the small and large intestine. Table 7 reports blood levels in infants and adults resulting from typical dietary exposures, and Table 8 reports blood levels in studies where humans were fed soy-based foods or supplements.

Infants are able to absorb isoflavones, and infants fed soy formula were shown to have plasma total isoflavone blood levels exceeding those of Japanese adults several-fold (Table 7) (Setchell et al., 1998). Higher concentrations in infants are postulated to result from lower body weight, frequent feedings, and reduced metabolism.

Studies examining the bioavailability [appearance of aglycone in plasma] of isoflavones ingested as glycosides versus aglycones have reported conflicting findings (UK Committee on Toxicity, 2003). Although some studies reported that bioavailability is increased when isoflavones are ingested as aglycones compared to glycosides, other studies found no differences in bioavailability. One study reported that genistein levels peaked a mean of $6.6 \mathrm{hr}$ after ingestion of the aglycone compared to $9 \mathrm{hr}$ when ingested as glycosides (Setchell et al., 2001). Bioavailability, as determined by area under the time-concentration curve (AUC), was reported to be greater for the glycosides than the aglycones. In a second study, Xu et al. (2000) observed that bioavailability of isoflavones did not vary when they were administered to women as foods containing a high percentage of aglycones (e.g., tempeh) compared to a high percentage of glycosides (e.g., soybeans) but noted that a study by Hutchins et al. (1995) found increased bioavailability for the aglycones. [The Expert Panel notes that bioavailability in pharmacology and toxicology refers to the ratio of AUC after oral ingestion to the AUC after intravenous (i.v.) injection. The term bioavailability is often used in different ways by different authors.]

A study by Xu et al. (2000) found that increases in dietary fat, protein, and cholesterol had no effect on bioavailability of isoflavones administered three times daily to eight women through powdered soy milk. Nevertheless, it has been postulated that bioavailability may be affected by food matrix, inter-individual variations, and intestinal microflora. Indeed, some studies reported that dietary fiber may lower isoflavone absorption (ILSI, 1999; UK Committee on Toxicity, 2003). A detailed evaluation of genistein and daidzein kinetics after ingestion of different soy foods was published by Cassidy et al. (2006). Men aged 18-55 years $(\mathrm{n}=21)$, premenopausal regularly-cycling women aged $18-53(\mathrm{n}=21)$, and postmenopausal women aged 48-69 $(n=17)$ were evaluated [only the men and premenopausal women will be considered here]. Subjects were given each of three soy foods separated by at least 2 weeks after which blood samples were drawn periodically over $72 \mathrm{hr}$ for measurement of total daidzein and genistein after hydrolysis. The foods were soy milk containing 15.2-19.6 $\mu \mathrm{g}$ daidzein aglycone equivalents and $22.3-28.3 \mu \mathrm{g}$ genistein aglycone equivalents, bread rolls made with textured vegetable protein containing $163.2 \mu \mathrm{g}$ daidzein aglycone equivalents and $312.9 \mu \mathrm{g}$ genistein aglycone equivalents, and tempeh burgers containing 72.4-137.1 $\mu \mathrm{g}$ daidzein aglycone equivalents and 140.3-186.4 $\mu \mathrm{g}$ genistein aglycone equivalents. As noted above, tempeh isoflavones are present largely as aglycones. The kinetic parameters were normalized by the isoflavone content of the food (Table 9). The authors of this study noted that isoflavones were absorbed more rapidly from liquid than from solid matrices and that absorption and AUC are higher when foods contain predominantly aglycones (tempeh burgers) as opposed to glycuronides (textured vegetable protein rolls).

Blood levels of genistein and daidzein (see Table 8) did not suggest saturated absorption in 12 women administered isoflavones at $0.7,1.3$, or $2.0 \mathrm{mg} / \mathrm{kg}$ bw through soy milk powder ( $44 \%$ genistein and 56\% daidzein) (Xu et al., 1994). 
Absorption half-lives for both daidzin and genistein, presumably as glucuronides and sulfates of daidzein and genistein, were reported at $\sim 1-3 \mathrm{hr}$ after intake of foods containing $\leq 210 \mathrm{mg}$ of each isoflavone or providing doses of $\leq 2 \mathrm{mg} / \mathrm{kg}$ bw/day of each isoflavone ( $\mathrm{Lu}$ et al., 1995; Lu and Anderson, 1998; Whitten and Patisaul, 2001). Genistein and daidzein equivalents were reported to peak at $\sim 6-8 \mathrm{hr}$ after ingestion of soy foods (Whitten and Patisaul, 2001; Pumford et al., 2002; UK Committee on Toxicity, 2003).

2.1.1.2 Distribution: One study reported volume of distribution for genistein and daidzein after intake of $50 \mathrm{mg}$ of the compounds as aglycones and glycosides (Setchell et al., 2001). After intake of each compound in its glycosidic form, the mean volume of distribution for the bioavailable fraction was reported at $\sim 161 \mathrm{~L}$ [ $2.7 \mathrm{~L} / \mathrm{kg}$ bw assuming a $60 \mathrm{~kg}$ bw] for genistein and $\sim 236 \mathrm{~L}[3.9 \mathrm{~L} / \mathrm{kg} \mathrm{bw}$ ] for daidzein. Ingestion of the glycosidic compounds resulted in volumes of distribution for the bioavailable fraction of $\sim 112 \mathrm{~L}[1.9 \mathrm{~L} / \mathrm{kg} \mathrm{bw}]$ for genistein and $77 \mathrm{~L}$ [1.3 L/kg bw] for daidzein. Similar mean volumes of distribution were reported for total genistein and daidzein in male and female volunteers who consumed $1-16 \mathrm{mg} / \mathrm{kg}$ bw formulations containing 70-100\% unconjugated isoflavones; the volumes of distribution were $\sim 1-6 \mathrm{~L} / \mathrm{kg}$ bw for genistein and $\sim 1-3 \mathrm{~L} / \mathrm{kg}$ bw for daidzein (Bloedon et al., 2002; Busby et al., 2002). In the same studies, volumes of distribution were higher for free isoflavones, $\sim 16-$ $877 \mathrm{~L} / \mathrm{kg}$ for genistein and $15-245 \mathrm{~L} / \mathrm{kg}$ bw/day daidzein. The study authors noted that the higher volumes of distribution for the free versus total isoflavones suggest that free genistein and daidzein are more likely to enter or be sequestered within tissues. [There are problems in the reporting of volumes of distribution for the aglycones in these studies. The Expert Panel finds ranges of 16-877 and 15-245 L/kg bw/day to be implausible in this variability. In addition, volumes of distribution in the range of $<10 \mathrm{~L} / \mathrm{kg}$ bw in some reports are not consistent with volumes of distribution in the hundreds of $\mathrm{L} / \mathrm{kg}$ bw in other reports. It is possible that the lower values were obtained from a mixture of aglycones and glucuronides with glucuronides predominating. The higher number may not take into consideration the bias toward the conjugated compounds in equilibrium.]

Adlercreutz et al. (1999) used a GC-MS method to measure maternal plasma, cord plasma, and amniotic fluid phytoestrogen levels in seven healthy omnivorous Japanese women (20-30 years old) who had just given birth. Results for isoflavones and two metabolites are listed in Table 10, with total levels of isoflavone levels reported for maternal blood and unconjugated and conjugated levels reported for cord plasma and amniotic fluid. Conjugated and unconjugated values were not reported for maternal plasma because the authors stated that patterns in plasma are well established. Isoflavone and metabolite levels were variable between subjects, and three subjects had very low levels of equol. Levels of isoflavones and metabolites in cord blood and amniotic fluid were similar to maternal cord blood levels. Pearson correlations are shown in Table 11. Levels of $O$-demethylangolensin and equol in maternal plasma correlated significantly with levels in cord plasma and amniotic fluid. Significant correlations between cord plasma and amniotic fluid were found for all the isoflavones and metabolites. Lignans were also detected in maternal and fetal compartments but at much lower levels. The authors concluded that phytoestrogens cross the placenta. Levels in fetuses were similar to maternal levels, and conjugation patterns were reportedly similar to those observed in Japanese individuals.

Foster et al. (2002b) measured phytoestrogens in 57 human amniotic fluid samples collected between 15 and 23 weeks of gestation. Samples were collected in Los Angeles [ethnic composition and dietary factors not discussed]. Measurements were made by GC/MS after glucuronidase treatment to hydrolyze the conjugates. Genistein equivalents were measurable in 42 of the samples with a mean $\pm \mathrm{SD}$ concentration of $1.08 \pm 0.91 \mathrm{ng} / \mathrm{mL}[\mathbf{4 . 0} \pm \mathbf{3 . 4} \mathbf{n M}$ ] (range $0.4-4.86 \mathrm{ng} / \mathrm{mL}$ [1.5-17.9 $\mathbf{~ M M}$ ). In a different study (Foster et al., 2002a), these authors reported genistein concentrations in 59 amniotic fluid samples obtained from 53 pregnant 
women at 15-23 weeks of gestation (four sets of twins and one woman who was sampled three times). There were 42 women with measurable amniotic fluid genistein concentrations. The mean \pm SD genistein equivalents concentration was $1.69 \pm 1.48 \mathrm{ng} / \mathrm{mL}[\mathbf{6 . 2 5} \pm \mathbf{5 . 4 8} \mathbf{~ n M}]$ (maximum $6.54 \mathrm{ng} / \mathrm{mL}$ [24.2 $\mathbf{~ n M ] ) . ~ [ I n ~ a ~ t a b l e , ~ t h e ~ m e a n ~} \pm$ SD is reported as $\mathbf{1 . 3 7 \pm 1 . 0 0 ~} \mathbf{n g}$ / $\mathrm{mL}(5.07 \pm 3.7 \mathrm{nM})$ with a median of $0.99 \mathrm{ng} / \mathrm{mL}(3.7 \mathrm{nM})$. It is not known whether there are any samples represented in both articles.]Engel et al. (2006) measured genistein and daidzein in amniotic fluid samples obtained before 20 weeks. The samples were collected for the sole indication of "advanced maternal age" (>35 years). The median (range) genistein concentration was $1.38(0.20-7.88) \mu \mathrm{g} / \mathrm{L}$ and the median (range) daidzein concentration was 9.52 (3.84-17.4) $\mu \mathrm{g} / \mathrm{L}$.

Franke et al. (1998) and Franke and Custer (1996) measured isoflavone and metabolite levels in the milk and urine of two women who consumed 5, 10, and $20 \mathrm{~g}$ roasted soybeans at 0,24 , and $72 \mathrm{hr}$ of the study, respectively. Plasma levels were also examined in one study (Franke et al., 1998). In the first study (Franke and Custer, 1996), isoflavone levels were measured in soybeans and found to be (in aglycone equivalents): daidzein $830 \mathrm{mg} / \mathrm{kg}$ soybean, genistein $913 \mathrm{mg} / \mathrm{kg}$ soy-bean, and glycitein $174 \mathrm{mg} / \mathrm{kg}$ soybean. Authors estimated the three daidzein doses at $0.08,0.15$, and $0.30 \mathrm{mg} / \mathrm{kg}$ bw and the three genistein doses at $0.08,0.17$, and 0.33 $\mathrm{mg} / \mathrm{kg}$ bw. In the second study (Franke et al., 1998), the soybeans reportedly contained daidzein $0.85 \mathrm{mg} / \mathrm{kg}$ soybean and genistein $1.1 \mathrm{mg} / \mathrm{kg}$ soybean, and the authors stated that consumption of $20 \mathrm{~g}$ soybeans would result in intake of $37 \mathrm{mg}$ isoflavones. [Based on the authors' value for isoflavone intake, it seems that the units of isoflavones in soybeans should have been $\mathrm{mg} / \mathrm{g}$ soybean instead of $\mathrm{mg} / \mathrm{kg}$ soybean, consistent with data reported for mature soybeans in the USDA (2002b) survey. Assuming that the correct unit for the isoflavones is $\mathrm{mg} / \mathrm{g}$ soybean, $20 \mathrm{~g}$ soybeans would contain $\sim 17 \mathrm{mg}$ total daidzein and $22 \mathrm{mg}$ total genistein.] Milk samples were collected each time the infant was nursed, and blood samples were drawn in one study after an overnight fast. Isoflavones and metabolites were hydrolyzed enzymatically, extracted from samples using methanol or ethyl acetate, and analyzed by HPLC.

In both studies, isoflavone levels in milk were below the detection limit (1-3 pM) before hydrolysis, suggesting that isoflavones occur in milk as glucuronide and sulfate conjugates. Soybean intake resulted in a rapid and dose-dependent increase in genistein and daidzein derivatives in milk. Concentrations peaked 10-14 hr after consumption of soybeans and returned to baseline levels 2-4 days later. A biphasic pattern of isoflavone detection was observed in milk after consumption of $20 \mathrm{~g}$ soybeans. Milk samples did not contain glycitein or the metabolites equol and $O$-demethylangolensin at detectable levels. Milk contained higher concentrations of genistein than daidzein conjugates. Levels of genistein conjugates were also higher in plasma [data were not shown]. In urine, the ratio of isoflavones to metabolites suggested preferential excretion of metabolites [data were not shown]. In contrast to results for milk and plasma, urinary conjugates of daidzein were higher than conjugates of genistein. Glycitein and the isoflavone metabolites equol and $O$-demethylangolensin were also detected in urine.

In the Franke and Custer (1996) study, intake of 5, 10, and $20 \mathrm{~g}$ soybeans by a single subject resulted in maximum milk total genistein levels of 32, 46, and $71 \mathrm{nM}$ and total daidzein levels of 16,34 , and $62 \mathrm{nM}$, respectively. The respective maximum urinary excretion rates for total genistein were 60,300 , and $300 \mathrm{nmol} / \mathrm{hr}$ and for total daidzein, 150, 500, and $600 \mathrm{nmol} / \mathrm{hr}$. Milk and urine levels were compared to those of a Chinese woman who ate her usual diet, which included one serving/day of tofu soup. The woman's milk contained total genistein at $30-50 \mathrm{nM}$ and total daidzein at $80-110 \mathrm{nM}$; urinary excretion rates ranged from $8-33 \mathrm{nmol} /$ $\mathrm{hr}$ for total genistein and from $80-150 \mathrm{nmol} / \mathrm{hr}$ for total daidzein. In the Franke et al. (1998) study, mean total isoflavone concentrations after consumption of $20 \mathrm{~g}$ soybeans were $0.2 \mu \mathrm{M}$ 
in milk and $2 \mu \mathrm{M}$ in plasma; urinary excretion rate for isoflavone was $3.0 \mu \mathrm{mol} / \mathrm{hr}$. [The data for "total isoflavones" were presented in the study abstract.]

A mean $\pm \mathrm{SD}$ isoflavone level of $5.6 \pm 4.4 \mu \mathrm{g} / \mathrm{L}$ was reported in milk samples from nine healthy lactating women (Setchell et al., 1998). Equol was detected in seven of nine milk samples. A $\leq 10$-fold increase in milk isoflavone levels was observed in a woman who ate $30 \mathrm{mg}$ isoflavones in the form of $10 \mathrm{~g}$ toasted soy nuts. [Figure 2 in the study report suggests that milk total daidzein levels increased $>15$-fold and total genistein levels increased $<10$-fold 1 day after the ingestion of the soy nuts.] The study authors noted that isoflavones occur predominantly as glucuronide conjugates in human milk. A second study reported that levels of total genistein and total daidzein were below the detection limit $(0.1 \mu \mathrm{g} / \mathrm{g})$ in milk samples from 11 mothers in New Zealand (Irvine et al., 1998a).

Isoflavone levels were measured in breast tissue from four women eating bread rolls supplemented with isoflavones $45 \mathrm{mg}$ for 14 days before undergoing breast biopsy (Pumford et al., 2002). Results were compared to those from four women who did not receive soy supplementation before biopsy. In women receiving soy supplementation, breast tissue contained total genistein 0.655 (range $=0.571-0.723) \mathrm{nmol} / \mathrm{g}$ wet tissue and total daidzein 0.145 (range $=0.083-0.128) \mathrm{nmol} / \mathrm{g}$ wet tissue. Breast tissue from women not undergoing supplementation contained total genistein $0.120($ range $=0.103-0.142) \mathrm{nmol} / \mathrm{g}$ wet tissue and total daidzein 0.021 (range $=0.017-0.028) \mathrm{nmol} / \mathrm{g}$ wet tissue .

Studies in humans reported that daidzein concentrations in prostate fluid were $~ 2-4$ times higher than plasma levels and equol concentrations in prostate fluid were 44 times higher than in plasma (Whitten and Patisaul, 2001).

2.1.1.3 Metabolism: The complete metabolic fates of genistein and daidzein are not known, but some information is available (Fig. 2, Fig. 3). Most of the information was obtained from reviews describing metabolism of iso-flavones after soy intake. However, some information obtained in studies with daidzein aglycone is also briefly discussed.

Metabolism of isoflavone glycosides begins with hydrolysis of the compounds to their respective aglycones, a step that must occur before the compounds can enter the systemic circulation. According to the UK Committee on Toxicity (2003), there is disagreement about whether hydrolysis occurs in the stomach. Intestinal bacteria such as Lactobacilli, Bifidobacteria, and Bacteroides contain $\beta$-glycosidase activity that may facilitate the hydrolysis of isoflavone glycosides, but the specific types of bacteria involved have not been identified. Bacterial flora is scarce in the small intestine but abundant in the large intestine. One study suggested that enzymes in saliva can hydrolyze isoflavone glycosides. $\beta$ Glucosidases in foods can also contribute to hydrolysis of glycosides (Kurzer and Xu, 1997), but their contribution to overall hydrolysis of the glycosides is minor.

Before entering the systemic circulation, the majority of genistein and daidzein are further metabolized to their conjugates, as noted in Figure 2 and Figure 3 (Joannou et al., 1995; Kurzer and $\mathrm{Xu}, 1997$; UK Committee on Toxicity, 2003). It is thought that additional metabolites can be generated both by intestinal and liver enzymes and by intestinal microflora. The metabolic profile has been reported to vary among individuals. Some individuals produce little or no equol or $O$-demethylangolensin.

Genistein and daidzein are conjugated with glucuronic acid by uridine diphosphateglucuronosyltransferase (UDPGT) or to a much lesser extent, with sulfate by sulfotransferases. In a study described in greater detail in the CERHR Expert Panel Report on Genistein (Rozman et al., in press), the study authors concluded that the intestine plays a major role in the glucuronidation of genistein (Doerge et al., 2000). In studies conducted with daidzein, the 
ability to glucuronidate daidzein was the same for microsomes from kidney and liver, whereas microsomes from colon had no measurable activity (Doerge et al., 2000). The 1A10 isoform of UDPGT, which is present in the gut but not in liver, lacked activity toward daidzein, whereas the 1A1 and 1A9 isoforms, found in gut and liver, had the greatest activity. Thus, study authors concluded that most daidzein glucuronidation occurs in liver (Doerge et al., 2000).

The glucuronide and sulfate conjugates enter the systemic circulation, and it has been reported that most isoflavones in the circulation are present in conjugated form. In individuals who ingested a soy supplement consisting of isoflavone glycosides, glucuronides represented 69$98 \%$ of circulating genistein and $40-62 \%$ of circulating daidzein (Doerge et al., 2000). In the same study, sulfate conjugates represented $4 \%$ of circulating daidzein compounds in one male but were not detected in a female subject. Genistein sulfates were not detected in the male or female. A second study reported mean steady-state circulating unconjugated isoflavones at $2.7 \%$ for daidzein and $1.6 \%$ for genistein when the compounds were taken as aglycones or glycosides (Setchell et al., 2001). The conjugated isoflavones undergo enterohepatic circulation, and on return to the intestine, they are deconjugated by bacteria with $\beta$ glucuronidase or aryl-sulfatase activity. The metabolites may be reabsorbed or further metabolized by gut microflora.

Limited ability of infants to produce equol was shown by Setchell et al. $(1997,1998)$, who detected equol in plasma of four of seven infants fed soy milk formulas but with a peak area one to two orders of magnitude lower than in infants fed cow-milk formula. Equol was detected in cow-milk samples from Australia (King et al., 1998). The finding was consistent with previous studies that found no equol in urine of infants fed soy formula and others that reported age-related increases in $\beta$-glucosidase activity (Setchell et al., 1998).

The ability of infants to absorb and metabolize isoflavones was shown by Hoey et al. (2004). The study examined 60 infants and children, aged 4 months to 6 years, who were assigned to either a soy-formula or control group $(\mathrm{N}=30$ in each) depending on whether they had consumed soy or cow-milk formula during infancy. Subjects were also divided into four groups according to age (4-6 months, 7-12 months, 1-3 years, and 3-7 years). Genistein, daidzein, and glycitein were present in the urine of all soy-fed infants in the 4-6-month age group, whereas $O$ demethylangolensin and equol were detected in $75 \%$ and $25 \%$ of soy-fed infants, respectively. In contrast, isoflavonoids were very low or not detected in the 4-6-month control group. In subjects aged 7 months to 7 years who were given a soy challenge, $O$-demethylangolensin was present in the urine of $75 \%$ of soy subjects and $50 \%$ of control subjects, whereas equol was present in the urine of $19 \%$ of soy subjects and $5 \%$ of control subjects. These percentages were similar between groups in the 3-7-year age group. Analysis of fecal samples showed that the total bacterial count was significantly higher in cow milk-fed infants than in the soy formulafed infants. More specifically, the bacterial counts for Bifidobacteria, Bacteroides, and Clostridia were significantly higher in the cow milk-fed group, whereas the combined count for Lactobacillus + Enterococcus was not significantly different between groups. There were no significant differences between the soy-formula and control groups in fecal enzyme activities, $\mathrm{pH}$, or short-chain fatty acid concentrations. The researchers concluded from the 46-month age group data that the isoflavones genistein, daidzin, and glycitein were well absorbed after hydrolysis in the gut because significant concentrations of their glucuronides were found in urine samples. They also deduced that the ability to hydrolyze glycosides to aglycones developed by the age of 4-6 months because such hydrolysis is required for absorption. Although a higher percentage of soy formula-fed infants than cow milk-fed infants of the younger age groups were able to convert daidzein to equol, the percentages were similar among the older children. Thus, the authors concluded that isoflavone exposure early in life has no lasting effect on isoflavone metabolism. They also noted the influence of formula type on the composition of the microflora present in the gut of infants. 
Setchell et al. (2001) reported that no adult females administered daidzein produced equol but that two of four women given daidzin produced equol; the study authors speculated that daidzin may have traveled further down the intestinal tract, where it was metabolized by bacteria. Halflives for equol formation were reported at 2-4 hr in adults eating soy foods (Lu et al., 1995; $\mathrm{Lu}$ and Anderson, 1998). A review by Price and Fenwick (1985) reported that most equol was excreted as glucuronide, with smaller amounts excreted as the sulfate conjugate in some humans.

Setchell (1998) reported that recent studies conducting detailed qualitative analysis of human urine identified numerous diphenolic metabolites generated in intermediates steps of genistein and daidzein biotransformation. At the time the Setchell review was published, the intermediate metabolites had not yet been identified by MS.

Mean recoveries of genistein and daidzein within $24-48 \mathrm{hr}$ are well below $100 \%$, as outlined in Table 12. Incomplete recoveries suggest the formation of additional metabolites such as $p$ ethylphenol or 2,4,6-tri-hydroxybenzoic acid (Steer et al., 2003). In some reviews such as the one conducted by Whitten and Patisaul (2001), recoveries were considered to represent bioavailability. Because recovery of daidzein and its metabolites usually exceeds that of genistein and its metabolites, it has been concluded that daidzein is more bioavailable than genistein; however, this conclusion is not supported by blood data that sometimes indicate higher levels of total genistein than total daidzein (Table 7,Table 8). Two studies by Setchell et al. $(2001,2003)$ reported greater bioavailability of genistein and derivatives than of daidzein and its metabolites based on blood levels measured over time; the authors noted that some studies reporting greater bioavailability of daidzein measured only two time points in the elimination period and could have reached erroneous conclusions. Setchell et al. (2003) also noted that urinary daidzein concentrations are consistently higher than genistein glucuronide concentrations when the two compounds are administered at equimolar concentrations, which is expected because of the greater plasma clearance rate of daidzein. In two trials of a study where $0.4 \mathrm{mg} / \mathrm{kg}$ bw of the ${ }^{13} \mathrm{C}$-labeled compounds were administered as aglycones to two different groups of eight women, the mean \pm SEM plasma clearance rates were reported at 26.73 $\pm 4.29 \mathrm{~L} / \mathrm{hr}$ for daidzein and $20.17 \pm 3.50 \mathrm{~L} / \mathrm{hr}$ for genistein.

There is some evidence that cytochrome P450 (CYP) may be involved in the metabolism of isoflavones. Unidentified metabolites considered to be hydrolysis products have been detected after in vitro incubation of genistein with human recombinant CYP1A1, 1A2, 1B1, 2E1, or 3A4 isoforms (UK Committee on Toxicity, 2003).

The role of gut microflora in the metabolism of isoflavones has been clearly established (UK Committee on Toxicity, 2003). Experiments conducted with cultured human fecal bacteria showed the formation of equol from textured vegetable protein and the conversion of daidzein to dihydrodaidzein, benzopyran-4,7-diol, 3,-(4-hydroxyphenol), and equol. Information on genistein metabolites is provided in the CERHR Expert Panel Report on Genistein (Rozman et al., in press).

In a review, Munro et al. (2003) reported that variations in metabolic pathways of isoflavones can occur as a result of differences in microflora, intestinal transit time, $\mathrm{pH}$, or redox potential, factors that can be affected by diet, drugs, intestinal disease, surgery, and immunity.

2.1.1.4 Excretion: Reviews that primarily addressed isoflavone exposure through soy product intake reported that most ingested genistein is excreted in urine, with very little excreted in feces (reviewed in ILSI, 1999; UK Committee on Toxicity, 2003). In studies in which volunteers ate soy-based foods, isoflavone excretion was reported at 30\% in urine and $1-4 \%$ in feces (Xu et al., 2000). An additional study in which volunteers drank soy milk reported 
peak urinary excretion at 8-10 hr, with $95 \%$ of excretion occurring within $24 \mathrm{hr}$; total urinary excretion consisted of $1 \%$ aglycones and $99 \%$ glucuronidated metabolites (Lu and Anderson, 1998). A second study reported that after ingestion of isoflavone mixtures containing $\geq 70 \%$ aglycones, $<0.3 \%$ of the dose was excreted as free genistein or daidzein (Bloedon et al., 2002).

Elimination half-lives were reported at 4-6 hr for genistein glucuronide, 3-4 hr for daidzein glucuronide, and 5-10 hr for equol after intake of $\leq 100 \mathrm{mg}$ of each isoflavone through soy milk (Lu et al., 1995). Studies in which male and female volunteers consumed formulations containing 70-100\% unconjugated isoflavones at $1-16 \mathrm{mg} / \mathrm{kg}$ bw reported longer mean halflives for total isoflavones ( $\sim 6-13 \mathrm{hr}$ for genistein and 4-16 hr for daidzein) compared to free isoflavones ( 2-7 hr for genistein and daidzein) (Bloedon et al., 2002; Busby et al., 2002). [A value of $21.2 \mathrm{hr}$ for free daidzein seemed to be an outlier.]

Numerous studies showed that urinary excretion of isoflavones increased with increasing soy product intake (UK Committee on Toxicity, 2003). However, one study showed that percent excretion of total genistein and daidzein was not affected at isoflavone levels of $0.7,1.3$, or $2.0 \mathrm{mg} / \mathrm{kg}$ bw/day administered through soy milk powder (Xu et al., 1994). Mean in vitro fecal degradation half-lives for 14 volunteers were reported at $\sim 8.9 \mathrm{hr}$ for genistein and $\sim 15.7 \mathrm{hr}$ for daidzein (Zhang et al., 1999).

It has been reported that urinary levels of daidzein and genistein derivatives were slightly lower in infants compared to adults fed equivalent amounts of isoflavones. This difference could indicate slower renal clearance in early life or slower glucuronidation and as a consequence, decreased renal secretion (Setchell et al., 1998).

Irvine et al. (1998b) measured urinary excretion of genistein and daidzein in four infants fed soy formula. The soy formulas contained genistein equivalents at $81-92 \mathrm{mg} / \mathrm{kg}$ formula and daidzein equivalents at $44-55 \mathrm{mg} / \mathrm{kg}$ formula as determined by HPLC. Based on measured isoflavone levels, recommendations by formula manufacturers, and infant weights, the authors estimated that the infants received isoflavones $2.9-3.8 \mathrm{mg} / \mathrm{kg}$ bw/day from 2-16 weeks of age. Once per week until 8 weeks of age and once every 2 weeks up to 16 weeks of age, 3-5 used diapers not containing feces were collected over a 24-hr period. Urine was diluted with water, squeezed from diapers, and pooled over $24 \mathrm{hr}$. Samples were hydrolyzed, methanol-extracted, and analyzed by HPLC. Recovery of isoflavones from diapers was verified. Data were presented as mean \pm SEM and analyzed by ANOVA. Average concentrations of isoflavones in urine were daidzein equivalents $2.9 \pm 0.3 \mathrm{mg} / \mathrm{L}$ and genistein equivalents $1.5 \pm 0.2 \mathrm{mg} / \mathrm{L}$. Mean percentages of daily isoflavone intake detected in urine and normalized for creatinine levels were daidzein equivalents $38 \pm 4 \%$ and genistein equivalents $13 \pm 3 \%$. Percent excretion was not affected by age. Isoflavone excretion rates varied little between infants and ranged from 0.37 \pm 0.03 to $0.58 \pm 0.06 \mathrm{mg} / \mathrm{kg}$ bw/day for daidzein and from $0.15 \pm 0.03$ to $0.32 \pm 0.04 \mathrm{mg} / \mathrm{kg} \mathrm{bw} /$ day for genistein. Age did not affect excretion rate. Urine from 25 infants fed dairy-based formula was also analyzed, and no isoflavones were detected [detection limit not specified].

2.1.2 Experimental animal-Placental and lactational transfer of dietary isoflavones in rats was examined in a developmental toxicity study that is discussed in detail in Section 3.2 (Weber et al., 2001a). Throughout pregnancy and lactation, 10 Sprague-Dawley rats received a commercially available high-phytoestrogen diet. The diet contained phytoestrogens $603 \mu \mathrm{g} /$ $\mathrm{g}$ feed consisting mainly of daidzin, genistein, and glycitein. The diet was soy-based, according to Lephart et al. (2002). At gestation day (GD) 20.5 [day of plug not specified] and on postnatal day (PND) 3.5 [day of birth not specified], blood was collected and pooled from dams and offspring ( $\mathrm{n}=3-5$ litters at each collection period) for measurement of total plasma genistein, daidzein, and equol levels by GC-MS. Blood levels of phytoestrogens in adult male rats fed 
the same diets were also examined. As noted from the study results listed in Table 13, gestational and lactational transfer of isoflavones was shown. Gestation day 20.5 dams were noted to have lower phytoestrogen plasma levels than male rats. The study authors proposed that changes in phytoestrogen metabolism or increased circulatory volume in late pregnancy were possible reasons for the lower plasma phytoestrogen levels in GD 20.5 dams.

Brown and Setchell (2001) measured serum levels of isoflavones in adult mice and rats and in fetuses and pups of rats fed various rodent diets. In adult FVB mice fed one of three soy-based diets (Purina 5008 or 5010 or NIH-07), serum levels of total genistein and daidzein [ $\leq \mathbf{1 0 0} \mathbf{~ n g / ~}$ $\mathrm{mL}$ each; $\leq \mathbf{3 7 0} \mathbf{~ n M}$ genistein, $\leq \mathbf{3 9 3} \mathbf{n M}$ daidzein] were about an order of magnitude lower

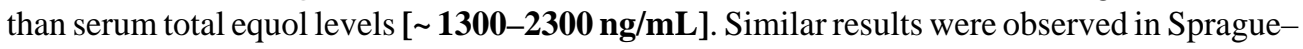
Dawley rats fed a soy-based diet (Purina 5001), with serum levels of total equol [ $\sim 2100 \mathbf{~ n g}$ / $\mathbf{m L}]$ greatly exceeding those of total genistein and daidzein $[\sim 100 \mathrm{ng} / \mathbf{m L}$ each; $\leq 370 \mathrm{nM}$ genistein, $\leq \mathbf{3 9 3} \mathbf{n M}$ daidzein]. Urine levels of genistein and daidzein glucuronide were about $25 \%$ of equol glucuronide levels in mice and $50 \%$ of equol glucuronide levels in rats. Results of serum analysis in rat pups of dams fed soy-based diets are presented in Table 14. According to the study authors, serum isoflavones in newborn pups before nursing represent maternalfetal transfer during gestation. The authors stated that pups do not start eating feed until 1516 days of age, and therefore serum levels of isoflavones in pups before that time point were also of maternal origin. Equol levels were very high at birth and rapidly declined during the postnatal period. Stomach contents of newborn rats, presumed to be swallowed amniotic fluid, also contained high levels of isoflavones consisting of $44 \%$ genistein derivatives, $37 \%$ equol derivatives, and $19 \%$ daidzein derivatives.

Lund et al. (2001a) also reported serum total equol levels [ 3000-4000 ng/mL; 12,389-16,519 $\mathbf{n M}]$ that were about an order of magnitude higher than levels of total genistein $[\sim \mathbf{3 0 0}-\mathbf{5 0 0}$ $\mathbf{n g} / \mathbf{m L} ; 1110-1850 \mathrm{nM}]$ or daidzein $[\sim 300-400 \mathrm{ng} / \mathrm{mL} ; \sim 1180-1573 \mathrm{nM}]$ in adult rats fed soy-based diets containing phytoestrogens at $600 \mu \mathrm{g} / \mathrm{g}$ diet.

In rats fed a soy-based diet containing $\sim 30 \mathrm{ppm}$ each of genistein and daidzin, serum total genistein levels were measured at $\sim 620 \mathrm{nM}$ in females and $350 \mathrm{nM}$ in males (Chang and Doerge, 2000). Serum total daidzein concentrations were measured at $250 \mathrm{nM}$ in females and at $200 \mathrm{nM}$ in males.

Isoflavones were detected in brains of adult male rats fed a soy-based diet containing $600 \mathrm{ppm}$ phyto-estrogens (Lephart et al., 2001; Lund et al., 2001b). As noted in Table 15, total isoflavones were greatest in frontal cortex > cerebellum > amygdala > hippocampus. In hippocampus, isoflavone levels were similar to those of rats fed a phytoestrogen-free diet. In the frontal cortex and amygdala, the concentration of equol was $>$ genistein $>$ daidzein. In the cerebellum, genistein > daidzein > equol. The study authors stated that cerebellum and frontal cortex contained an abundance of estrogen receptor (ER) $\beta$. Levels of phytoestrogens in the medial basal hypothalamic and preoptic areas were reported at $4.4 \mathrm{ng} / \mathrm{g}[\mathbf{0 . 0 1 7} \mathbf{~ n m o l} / \mathbf{g}]$ daidzein, $3.5 \mathrm{ng} / \mathrm{g}[\mathbf{0 . 0 1 3} \mathbf{~ n m o l} / \mathbf{g}]$ genistein, and $126 \mathrm{ng} / \mathrm{g}[\mathbf{0 . 5 2} \mathbf{~ n m o l} / \mathrm{g}]$ equol (Lephart et al., 2001). Levels of genistein and equol were significantly higher than in rats fed a phytoestrogenfree diet.

Whitten and Patisaul (2001) reviewed animal toxico-kinetic data on genistein and daidzein derivatives (Table 16). In a review, Setchell (1998) reported that high levels of equol equivalents have been detected in portal venous blood and bile of rats, thus indicating that isoflavones undergo enterohepatic circulation. Because equol was primarily found as a conjugate of glucuronic acid in venous portal blood, it was suggested that conjugation within the intestinal wall may occur during absorption. Intestinal conjugation of genistein was confirmed in studies using everted rat intestinal preparations (Sfakianos et al., 1997); the study 
is described in detail in the CERHR Expert Panel Report on Genistein (Rozman et al., in press).

\subsection{General Toxicology}

2.2.1 Thyroid-Concerns about soy-induced thyroid toxicity arose in the 1930s when goiters were observed in rats fed soybeans (Fitzpatrick, 2000; UK Committee on Toxicity, 2003). In the 1950s and 1960s, cases of altered thyroid function, mostly goiter, were reported in infants fed soy formula. The problem was eliminated by adding more iodine to the formulas and replacing soy flour with soy protein isolate. Studies addressing possible thyroid toxicity resulting from soybean or isoflavone intake in developing humans or animals are discussed more fully in Section 3, whereas the remainder of this section focuses on effects in adults.

A study in Japanese men fed $30 \mathrm{~g}$ soybeans/day for 1 or 3 months reported signs of thyroid toxicity including increased thyroid-stimulating hormone levels, decreased thyroxine, and diffuse goiter (Fitzpatrick, 2000; UK Committee on Toxicity, 2003). Symptoms of constipation, fatigue, and lethargy were observed in about half the subjects treated for 3 months. Recovery was observed after cessation of the soybean diet. Based on levels of isoflavones in Japanese soybeans, Fitzpatrick (2000) estimated intakes of $23 \mathrm{mg} /$ day genistein and $10 \mathrm{mg} /$ day daidzein. Other studies in humans ingesting up to $132 \mathrm{mg} /$ day isoflavones, through soy consumption in most cases, reported small and variable effects on thyroid hormone levels that the study authors did not consider clinically significant (UK Committee on Toxicity, 2003). Both Fitzpatrick (2000) and the UK Committee on Toxicity (2003) expressed concerns that individuals with hypothyroidism may be more susceptible to thyroid effects after soy intake or that soy may interfere with medications used to treat the condition. Though limited, epidemiologic studies suggest no association between phytoestrogen exposure through soy consumption and thyroid cancer risk in humans.

Evidence of thyroid toxicity (e.g., increases in thyroid weight and thyroid-releasing hormone level, decreased thyroxine level, and histological changes) was observed in iodine-deficient rats fed soybeans, but there was no effect when rats were fed soy providing genistein concentrations of $60 \mathrm{mg} / \mathrm{kg}$ diet or isoflavones at $\leq 2000 \mathrm{mg} / \mathrm{kg}$ diet (UK Committee on Toxicity, 2003). One study of iodine-deficient rats fed soybeans reported an increase in thyroid carcinoma, but no evidence of carcinogenicity was observed in a second study examining effects of genistein intake ( $\leq 250 \mathrm{mg} / \mathrm{kg}$ diet) in rodents (UK Committee on Toxicity, 2003).

2.2.2 Cardiovascular-The FDA (1999) and the UK Committee on Toxicity (2003) reviewed numerous human studies examining the effects of soy foods on cholesterol levels. Based on well conducted human studies, both the FDA and UK Committee on Toxicity concluded that consumption of soy foods as part of a diet low in saturated fat and cholesterol can lower total blood cholesterol and low density lipoprotein levels. As noted in the Expert Panel Report on Genistein, there is no conclusive evidence that the hypocholesteremic properties of soy products are due to isoflavones. The UK Committee on Toxicity examined the effects of soy product intake on other risk factors associated with cardiovascular disease (e.g., high blood pressure, thrombosis, and atherosclerosis), and concluded that there are insufficient data to reach a conclusion. A study using a transgenic mouse model of hypertrophic cardiomyopathy (Stauffer et al., 2006) showed that feeding a soybean-based diet resulted in more dilated ventricles and poorer cardiac function than did feeding a casein based diet. This effect was seen only in males.

2.2.3 Menopausal symptoms-Some perimenopausal and menopausal women experience hot flashes and vaginal dryness; these symptoms can vary by culture (Kurzer and $\mathrm{Xu}, 1997)$. One study noted that fewer menopausal Japanese compared to Canadian women 
experienced hot flashes. It was postulated that weak estrogenic effects associated with a phytoestrogen-rich diet could be the cause of reduced menopausal symptoms in Japanese women. Therefore, the effects of soy diets and isoflavone supplements on hot flashes were investigated. Of 12 studies reviewed by the UK Committee on Toxicity (2003), half reported that soy diets or isoflavone supplementation reduced the frequency of hot flashes, whereas the other half reported no effect on hot flashes.

The effects of soy-product or genistein intake on vaginal cytology in postmenopausal women are reported in the discussion of in vivo estrogenicity effects.

Animal studies reviewed by the UK Committee on Toxicity (2003) showed consistently that soy products prevented bone loss in ovariectomized rodents. Epidemiologic studies reviewed by the UK Committee on Toxicity (2003) reported higher bone mass in populations consuming more soy products. A small number $(\sim 6)$ of short-term intervention studies in humans showed consistently small but statistically significant soy-associated increases in bone mineral density in the lumbar spine. Other studies examining effects at other sites produced equivocal findings, with some reporting beneficial effects on bone mineral density and others reporting no effect. The UK Committee on Toxicity stated that long-term studies are needed before conclusions can be made about the effectiveness of phytoestrogens in improving bone health.

\subsubsection{Estrogenicity}

2.2.4.1 Human: Estrogenic effects were examined in women receiving soy diets or supplements, summarized in Table 17. Three of the studies did not demonstrate changes in vaginal cytology related to soy product intake. In the more detailed of these studies (Baird et al., 1995;Duncan et al., 1999b), women were exposed to up to $1 \mathrm{mg} / \mathrm{kg}$ bw/day genistein for 30-93 days. A fourth study that provided very limited detail and included alternating exposure to non-soy-based phytoestrogens reported increased vaginal cell maturation after women received soy flour supplements for 2 weeks (Wilcox et al., 1990). A fifth study with a longer exposure period (5 years) showed estrogenic effects on the endometrium (Unfer et al., 2004). However, a limitation of the Unfer et al. (2004) study was noted in a letter to the editor by Foth and Nawroth (2005). They noted that at baseline and at the 30-month evaluation period $25 \%$ of endometrium samples were inaccessible in the treated and placebo groups. [The Expert Panel notes that 20-25\% of endometrium samples were also inaccessible at the 5-year evaluation period.] It does not seem that women with inaccessible endometrium samples at baseline were excluded for evaluation at future time points. Therefore, it is not known if endometrial hyperplasia was present at baseline in women with inaccessible endometrium samples. It was also noted that no information was provided about endometrial thickness or bleeding patterns. A sixth study reported increased proliferation of breast lobular epithelium and progesterone receptor expression in women who ate bread rolls containing $60 \mathrm{~g}$ soy supplement as textured vegetable protein (McMichael-Phillips et al., 1998). The final report (Hargreaves et al., 1999) with the full cohort of 84 individuals (including 33 added from a tissue bank) showed no differences between the control group and the group eating soy rolls other than plasma isoflavone levels.

2.2.4.2 Experimental animal: Ashby et al. (2000b) examined uterotropic effects of infant formulas. From PND 21/22 through 24/25, Alpk rats were given access to infant formula in drinking water bottles and RM1, a standard diet consisting of $6 \%$ soy protein. Three soy-based and one cow-milk formula were prepared as recommended by manufacturers. Rats fed the RM1 diet and not given infant formula served as negative controls, and rats given diethylstilbestrol or $17 \beta$-estradiol served as positive controls. Data were analyzed by analysis of covariance (ANCOVA). [It is not clear how many animals were examined in each group, but if numbers above the bars in a graph indicated the numbers of animals, then each 
group contained 5-29 animals.] Results are summarized in Table 18. Both cow-milk and soy-based formulas prepared at full strength increased uterine weight, with greater responses generally noted with recommended concentrations of soy compared to cow-milk formula. Testing of three different concentrations of one of the soy formulas (Infasoy®) showed doserelated responses. It was noted that rats consumed Infasoy at a level three times the recommended intake for an infant on a $\mathrm{mg} / \mathrm{kg}$ bw basis. The cow-milk formula, SMA Gold, and the 33\%-strength Infasoy formula were consumed at levels similar to those recommended for infants on a $\mathrm{mg} / \mathrm{kg}$ bw basis. Faslodex, an ER $\alpha$ and ER $\beta$ antagonist, inhibited the uterotropic effects of soy and cow-milk formulas and of diethylstilbestrol [data not shown for cow-milk formula]. The aromatase inhibitor anastrozole attenuated the uterotropic response of Infasoy but not of $17 \beta$-estradiol [data not shown]. The gonadotropin-releasing hormone (GnRH) antagonist antarelix inhibited the uterotropic effect of cow-milk and soy formulas but not of diethylstilbestrol [data not shown for cow-milk formula]. Neither the Infasoy nor cow-milk formula induced an uterotropic response in ovariectomized adult rats [data not shown]. Dietary effects on puberty were also examined and are discussed in Section 3. The study authors concluded that both cow-milk and soy formulas induced estrogenic effects in rodents independent of formula phytoestrogen content. The authors further stated that infants fed recommended quantities of formulas would consume similar quantities of formula (on a mg/ $\mathrm{kg}$ bw basis) as rats given the 33\% soy formula or cow-milk formula, resulting in exposures at the threshold of estrogenic activity in rats.

Results of estrogenicity testing in laboratory animals fed soy-based diets are summarized in Table 19. No evidence of estrogenicity was observed in studies in which rats or monkeys were fed soy-based diets (Cline et al., 1996;Tansey et al., 1998;Ashby et al., 2000a;Wood et al., 2004); however, estrogenic effects occurred when soy isoflavones were added to a soy-free diet (Wade et al., 2003). In the monkey studies (Cline et al., 1996; Wood et al., 2004), the genistein dose in the soybean extract was stated to be equivalent to that of a woman receiving genistein $99.7 \mathrm{mg} /$ day [ $\sim 2 \mathbf{~ m g} / \mathbf{k g ~ b w} / \mathbf{d a y}$ assuming a $50 \mathbf{~ k g ~ b w ]}$. A study in immature female mice found a soy-based diet to increase relative uterine weight and to antagonize the weight increase associated with feeding diethylstilbestrol, demonstrating weak estrogen agonist and antagonist properties (Mäkelä et al., 1995b). In a study in which rats were fed soy protein with and without the addition of an estrogen, there were no additive effects between soy diets and estrogen (Tansey et al., 1998). Soy diets that were not alcohol extracted to remove isoflavones antagonized the effects of estrogens on increased lactoferrin staining and epithelial luminal cell height. In some studies, increases in rodent uterine weight were greater in rats fed soy-free versus soy-containing diets (Thigpen et al., 1987,1999;Ashby et al., 2000a,b).

\subsection{Genetic Toxicity}

No soy formula genotoxicity studies were identified. Genotoxicity of genistein is discussed in the CERHR Expert Panel Report on Genistein (Rozman et al., in press).

\subsection{Cancer}

Studies examining cancer in humans or rodents exposed to soy during prenatal or postnatal development are discussed in Section 3. This section is limited to studies examining soy intake in adulthood and is based on reviews. Possible mechanisms by which genistein could modulate breast cancer effects were examined and are discussed in the CERHR Expert Panel Report on Genistein (Rozman et al., in press). Other components of soy that were suggested as having possible chemo-preventive properties include protease inhibitors, phytic acid, and $\beta$-sitosterol (Adlercreutz, 2002).

It has been observed that breast cancer rates are lower in Asian than Western populations (Bouker and Hilakivi-Clarke, 2000). Breast cancer rates are higher in Asian women who 
immigrate to the U.S. before age 35 and in Asian women born in the U.S. than in women born in Asian countries who do not immigrate to the U.S. Higher soy product intake in Asian women has been investigated as a possible reason for lower breast cancer rates in Asian populations. Several epidemiologic studies have been conducted to determine if soy product intake in adulthood is related to breast cancer incidence, and these studies were addressed in reviews (Adlercreutz, 2002; UK Committee on Toxicity, 2003). The reviews reported conflicting results for epidemiologic studies, with some studies demonstrating protective effects of soy product intake and others demonstrating no effect. A meta-analysis conducted by Bouker and Hilakivi-Clarke (2000) indicated that soy products may reduce breast cancer risk in premenopausal women but may have no effect in postmenopausal women. The UK Committee on Toxicity (2003) noted that most rodent studies examining links between soy product intake and chemically induced mammary cancer reported protective effects of soy product intake, but one study showed no effect. The final conclusion in each of the reviews was that there was no convincing evidence that soy products or isoflavones have beneficial or adverse effects on development of breast cancer (Adlercreutz, 2002; UK Committee on Toxicity, 2003). Bouker and Hilakivi-Clarke (2000) noted that evaluations of associations between soy product intake and breast cancer risks need to consider confounding factors such as timing and duration of exposure, interactions between different components of soybeans, and other environmental or lifestyle factors that may contribute to lower breast cancer rates in Asian women.

It was observed that Asian men have lower rates of invasive prostate cancer and mortality, although rates of latent, small, and non-infiltrative prostate cancer are similar in Asian and Western men (UK Committee on Toxicity, 2003). Studies examining the association between soy intake and prostate cancer in adults were reviewed by the UK Committee on Toxicity (2003) and Adlercreutz (2002). Studies of fermented soy intake by Asian men most often showed no associations, whereas studies of non-fermented soy consumption by Western men most often showed protective effects. Most of the rodent studies involved tumor implants or chemically induced cancers (UK Committee on Toxicity, 2003). In the majority of experimental animal studies, tumor growth was inhibited in animals fed soy (Adlercreutz, 2002; UK Committee on Toxicity, 2003). The UK Committee on Toxicity concluded that evidence of protective effects against prostate cancer is greater in experimental animals than in humans and noted that interpretation of human studies is complicated by confounding factors such as differing lifestyle and diet. In contrast Adlercreutz concluded that there was fairly strong evidence that a diet high in soy and isoflavones protects against prostate cancer.

According to the UK Committee on Toxicity (2003), human studies generally have indicated an increased risk of colorectal cancer with consumption of fermented soy products and a decreased risk of colorectal cancer with consumption of non-fermented soy products. Studies in experimental animals have been reviewed (Adlercreutz, 2002; UK Committee on Toxicity, 2003). Adlercreutz (2002) concluded that soy products did not seem to offer protection against colon cancer.

Genistein was postulated to have chemopreventive properties, and a discussion is included in the CERHR Expert Panel Report on Genistein (Rozman et al., in press). Other compounds with possible chemopreventive properties were identified in soy, and they include Bowman-Birk protease inhibitors (compounds that affect cell transformation), inositol phosphates, phytosterols, and saponins (Lu et al., 2000a). Ingestion of soy in the diet alters the urinary excretion pattern of estrone and $17 \beta$-estradiol metabolites, shifting the ratio of 2- and 16hydroxylated forms toward the more favorable 2-hydroxyestrone and 2-hydroxyestradiol (Xu et al., 1998; Lu et al., 2000b).

It has been postulated that soy protein isolate can reduce carcinogenicity of procarcinogens such as dimethyl benzanthracene by inhibiting CYP enzymes involved in bioactivation 
(Rowlands et al., 2001; Badger et al., 2002). Rats fed diets containing soy protein isolates compared to casein had reduced levels of CYP1A1 protein in liver and CYP1A1, CYP1A2, and CYP1B1 proteins in mammary gland. Similar reductions in CYP protein expression were observed in rats fed whey-based diets. Feeding of soy protein isolate to rats exposed to dimethyl benzanthracene lowered levels of dimethyl benzanthracene-deoxyribonucleic acid (DNA) adducts in tissues (Badger et al., 2002). Thus, it has been suggested that downregulation of CYP1A1 and CYP1B1 is a possible mechanism of cancer protection in dimethyl benzanthracene-treated rats. Soy protein diets treated to remove isoflavones also provided protection against dimethyl benzanthracene-induced cancer in rats, suggesting that the protective effects of soy are not due to isoflavones.

Reactive oxygen species can damage DNA, cellular proteins, and lipids and may be involved in carcinogenesis (UK Committee on Toxicity, 2003). Soy isoflavones have antioxidant properties. Studies in humans ingesting soy products with $\geq 12 \mathrm{mg}$ genistein or $\geq 56 \mathrm{mg}$ isoflavones showed reductions in oxidized low-density lipoprotein, lipoprotein peroxidation, or oxidative DNA base damage (UK Committee on Toxicity, 2003).

\subsection{Potentially Sensitive Subpopulations}

It has been reported that bacterial $\beta$-glucosidase is lower in infants compared to adults and increases with age (Setchell et al., 1998).

Production of equol was found to vary greatly among individuals, and it is estimated that only $30-40 \%$ of the human population is capable of producing equol, an estrogenic metabolite of daidzein (Setchell et al., 1984, 2003; Kurzer and Xu, 1997; Xu et al., 1998; Rowland et al., 2000; UK Committee on Toxicity, 2003). A study by Lu et al. (1995) reported that only one of six men produced equol after ingestion of soy milk. Higher levels of parent compound and precursor metabolites were detected in urine of low-equol metabolizers. By contrast, a study by Kelly et al. (1995) found equol to increase markedly in the urine of 11 of 12 women given soy flour, and a study by Cassidy et al. (1994) reported that equol was detected in five of six women fed soy protein, with high concentrations detected in two women. Excretion of equol and its precursor daidzein seemed to be inversely related but were not statistically evaluated. Setchell et al. (2002) reviewed the role of metabolism of daidzein or for-mononetin (a clover isoflavone) to equol in experimental animal and human intestine and argued that much of the estrogenic activity of dietary soy products was due to the equol. They indicated, for example, that the menstrual cycle study of Cassidy et al. (1994), reviewed in Section 3.1, showed the greatest effect of soy foods on menstrual cycle lengthening in the two women whose urinary equol excretion was high. The variability in human equol excretion after soy product intake has been attributed to variations in intestinal microflora, although the relevant microorganisms have not been identified (Rowland et al., 2000). As discussed above, Setchell et al. (1997; 1998) reported that infants fed soy formula excreted little, if any, equol, presumably due to immaturity of their bowel flora. The rat, by contrast, was characterized as an "equol-producing machine" because of its large cecum and abundant microflora (Setchell et al., 2002). Thus, susceptibility to the estrogenic effects of dietary soy products may vary from one individual to the next and may be low in infants fed soy formula. [These data suggest that the rat pup may not be a good model for the human infant.]

Some studies suggested that diet can influence equol production because increased levels of production were noted with a high-carbohydrate and low-fat diet. Variations in excretion of $O$-demethylangolensin, a metabolite of daidzein, and 6'-hydroxy- $O$-demethylangolensin, a metabolite of genistein, have also been reported.

A study by Lu and Anderson (1998) reported sex-specific differences in metabolism of isoflavones in soy milk. On study days $3,16,17,30$, and 31 , six men and six women living in 
a metabolic unit ingested $1.065 \mathrm{~L}$ soy milk after an overnight fast. With each dose, subjects received $80-210 \mathrm{mg}$ of each isoflavone ( $80 \%$ in glycosidic form). [Isoflavone levels were also reported as $34 \pm 15$ (SD) total daidzein and $38 \pm 15 \mathrm{mg}$ total genistein.] Urine was collected before and up to $48 \mathrm{hr}$ after each exposure. Recovery of the ingested dose was reported at $24 \%$ for genistein and its glycoside, $66 \%$ for daidzein and its glycoside, and $28 \%$ for equol and its glycoside in women and $15 \%$ for genistein and its glycoside, $47 \%$ for daidzein and its glycoside, and $15 \%$ for equol and its glycoside in men. Authors noted the higher excretion of daidzein in women compared to men. Over time, there was a progressive decrease in percent urinary recovery and shortened half-life in women but not men. Increased metabolism or cyclic variations in hormones were discussed as possible reasons for changes in urinary excretion in women. Elimination half-lives in women $(\sim 4.4 \mathrm{hr}$ for daidzein and its glycoside and $6.7 \mathrm{hr}$ for genistein and its glycoside) were longer than elimination half-lives in men $(\sim 2.9 \mathrm{hr}$ for daidzein and its glycoside and $3.8 \mathrm{hr}$ for genistein and its glycoside). One of six men and one of six women were capable of producing equol (16-28\% of daidzein intake) at the start of the study. Two weeks into the study, three additional women, but no additional men, developed the ability to produce equol.

A review by Setchell (1998) reported that some studies showed no sex-related differences in biotransformation and excretion of soy isoflavones.

As noted in the toxicokinetics section (Section 2.1), most genistein and daidzein is present in the circulation as glucuronide conjugates. Studies in humans suggest that infants may have decreased ability to glucuronidate isoflavones because UDPGT activity is low in the fetus and neonate but gradually increases to adult levels in the time period of months (Doerge et al., 2002). Coughtrie et al. (1988) examined the ontogeny of UDPGT in humans. Activity was measured in postmortem liver microsome samples obtained from premature or full-term infants and adults. Results of this analysis are listed in Table 20. Activities for isoenzymes catalyzing glucuronidation of bilirubin, testosterone, and 1-napthol were very low at birth in premature and full-term infants. Activities increased with age for the isoenzymes catalyzing glucuronidation of bilirubin ( 80\% of adult levels by $8-15$ weeks of age) and 1-naphthol ( $30 \%$ of adult levels at $8-15$ weeks of age). During the first 55 weeks of life, no consistent increase in activity was noted for the isoenzyme catalyzing glucuronidation of testosterone. Using an immunoblot technique with antibodies developed toward liver testosterone/4nitrophenol and kidney naphthol/bilirubin, one immunoreactive protein was observed in microsomes of 18- and 27-week-old fetuses, three immunoreactive proteins were observed in microsomes of term infants, and most isoenzymes present in adults were observed within 3 months of age at levels $\sim 25 \%$ those of adults.

Despite the possibility of lowered UDPGT activity in infants, a letter to the editor providing few details but a reference for the analytical method used reported no detectable levels of unconjugated isoflavones in plasma from four infants (2.5-5.5 months old) exclusively fed soy formula for at least 2 weeks (Huggett et al., 1997). Blood samples had been measured before and after hydrolysis with $\beta$-glucuronidase and sulfatase, but the percentages of each conjugate were not specified. [The Panel was not able to verify this information due to lack of experimental details and data. This reference is presented for completeness and will not be considered further.]

Coughtrie et al. (1988) also measured activity and expression of UDPGT in hepatic microsomes of WAG rats from GD 17 to PND 75. Consistent results were obtained using methods to measure enzyme activity and protein levels via immunoreactive probes. Activity of the isoenzyme catalyzing the glucuronidation of testosterone was barely detectable in fetuses, increased to $\sim 20 \%$ of adult levels at birth, and continued to increase until reaching adult levels between 26-30 days of age (with the exception of a decrease on PND 40). Activity of the 
isoenzyme catalyzing glucuronidation of bilirubin was barely detectable in fetuses, increased at birth to reach 75\% of adult levels on PND 2-16 (with the exception of a decrease on PND 5 ), and attained or exceeded adult levels by PND 20 (with the exception of a decrease on PND 40 ). The isoenzyme catalyzing glucuronidation of 2-aminophenol had $~ 30-60 \%$ of adult activity in fetuses, reached or exceeded adult activity on PND $2-5$, had $\sim 30 \%$ of adult activity on PND 10-20, and reached or exceeded adult activity by PND 26.

A study in rats examined the effects of intestinal microflora on interindividual variations in isoflavone metabolism (UK Committee on Toxicity, 2003). Feeding of soy protein to germfree rats lacking intestinal microflora resulted in urinary excretion of daidzein and genistein but not equol or $O$-demethylangolensin. When the rats were colonized with bacterial flora from a human able to convert daidzein to equol, the rats excreted equol but not $O$ -

demethylangolensin. No equol was excreted by the rats when they were colonized by bacteria from a human unable to produce equol.

\subsection{Summary of General Toxicology and Biological Effects}

2.6.1 Toxicokinetics and metabolism-This section focuses on toxicokinetics and metabolism of two isoflavones, genistein and daidzein, found mostly as glycosides in soy formula and other soy foods. Detailed toxicokinetic data on other soy isoflavones were not located. Studies that focus on toxicokinetics after exposure to pure genistein are discussed in the CERHR Expert Panel Report on Genistein (Rozman et al., in press), although some highly relevant information for genistein will be repeated in this report.

2.6.1.1 Humans: Genistein and daidzein exist mainly in their glycosidic forms in unfermented soy foods. Before isoflavone glycosides can be absorbed into the systemic circulation, they must first be hydrolyzed to their aglycones, which have greater hydrophobicity and lower molecular weights (UK Committee on Toxicity, 2003). The majority of isoflavones are glucuronidated before entering the systemic circulation (Doerge et al., 2000). Absorption half lives in adults for both daidzein and genistein (free + metabolites) were reported at $\sim 1-3 \mathrm{hr}$ after intake of foods containing $\leq 210 \mathrm{mg}$ of each isoflavone type or providing doses of $\leq 2 \mathrm{mg}$ / $\mathrm{kg}$ bw/day of each isoflavone type (Lu et al., 1995; Lu and Anderson, 1998; Whitten and Patisaul, 2001). Genistein and daidzein conjugates were reported to peak in adults at $\sim 6-8 \mathrm{hr}$ after ingestion of soy foods (Whitten and Patisaul, 2001; Pumford et al., 2002; UK Committee on Toxicity, 2003).

Genistein and daidzein (free + metabolites) were detected in blood of individuals who consume diets containing soy products (Table 7,Table 9) and volunteers consuming soy-based foods or supplements (Table 8). Infants are able to absorb isoflavones, and infants fed soy formula were shown to have plasma total isoflavone blood levels exceeding those of Japanese adults (Table 7) (Setchell et al., 1998). The highest total genistein/daidzein blood levels were reported for infants fed soy formula ( 2530/1160 $\mathrm{nM}[\mathbf{6 8 3} / \mathbf{2 9 5} \boldsymbol{\mu \mathrm { g }} / \mathrm{L}$ aglycone equivalents]), and those values exceeded blood levels reported for Asian populations ( 90-1200 / 60-900 nM [24324/15-229 $\mu \mathrm{g} / \mathrm{L}$ aglycone equivalents]). Total genistein / daidzein blood levels in infants fed human milk or cow-milk formula were reported at 10-12/6-8 nM [2.7- 3.2/1.5-2.0 $\mathbf{\mu g} /$ L aglycone equivalents]. In Finland and Canada, total genistein/daidzein blood concentrations were reported at $0.5-8 / 0.6-6 \mathrm{nM}[\mathbf{0 . 1 4 - 2 . 1 6 / 0 . 1 5 - 1 . 5} \boldsymbol{\mu g} / \mathrm{L}$ aglycone equivalents] in omnivores and 17-45/19- $50 \mathrm{nM}$ [4.6-12/4.8-13 $\mu \mathrm{g} / \mathrm{L}$ aglycone equivalents] in vegetarians. Higher concentrations in infants are postulated to result from lower body weight, frequent feedings, and reduced metabolism.

In a study by Hoey et al. (2004), the presence of total genistein, daidzein, and glycitein in urine samples of 4-6-month-old infants fed soy formula led study authors to conclude that isoflavones were well absorbed by infants. It was also deduced that the 4-6-month-old infants 
had the ability to hydrolyze glycosides to aglycones because such hydrolysis is required for absorption of isoflavones.

Blood levels of total genistein and daidzein did not suggest saturated absorption in 12 women administered soy milk (Table 8) (Xu et al., 1994).

Three studies detected genistein in human amniotic fluid at up to $212 \mathrm{nM}[\mathbf{0 . 2 0}-\mathbf{5 7} \boldsymbol{\mu g} / \mathbf{L}$ aglycone equivalents] (Adlercreutz et al., 1999; Foster et al., 2002b; Engel et al., 2006). One of the studies showed that $84 \%$ of total genistein in amniotic fluid and $91 \%$ in cord blood was present as a glucuronide conjugate (Adlercreutz et al., 1999). Studies by Franke and Custer (1996) and Franke et al. (1998) reported distribution of genistein and daidzein to human milk. Total isoflavone concentrations peaked $10-14 \mathrm{hr}$ after consumption of soybeans and returned to baseline levels $2-4$ days later. Total isoflavone levels in milk were below the detection limit (1-3 pM) before hydrolysis, suggesting that isoflavones occur in milk as glucuronide and sulfate conjugates. In the Franke and Custer (1996) study, intake of 5, 10, and $20 \mathrm{~g}$ soybeans by a single subject resulted in maximum milk total genistein levels of 32, 46, and $71 \mathrm{nM}[\mathbf{8 . 6}$, 12 , and $19 \mu \mathrm{g} / \mathrm{L}$ ] and total daidzein levels of 16,34 , and $62 \mathrm{nM}[4.1,8.6$, and $16 \mu \mathrm{g} / \mathrm{L}]$. Milk total isoflavone levels in a Chinese woman who ate one serving of tofu soup a day were reported at 30-50 $\mathrm{nM}$ genistein equivalents [8.1-14 $\boldsymbol{\mu g} / \mathbf{L}$ ] and 80-110 $\mathrm{nM}$ daidzein equivalents [20$28 \mu \mathrm{g} / \mathrm{L}]$. A third study reported a mean $\pm \mathrm{SD}$ total isoflavone level of $5.6 \pm 4.4 \mathrm{mg} / \mathrm{L}$ in milk samples from nine healthy lactating women (Setchell et al., 1998). Total equol was detected in seven of nine milk samples.

Metabolism of genistein is outlined in Figure 2, and metabolism of daidzin is outlined in Figure 3. Before absorption, most genistein and daidzein are conjugated with glucuronic acid by UDPGT in the gut wall; a smaller amount is conjugated to sulfate by sulfotransferases (Joannou et al., 1995; Kurzer and Xu, 1997;UK Committee on Toxicity, 2003). Conjugation of isoflavones can also occur in liver. The glucuronide and sulfate conjugates enter the systemic circulation, and the majority of isoflavone compounds in the circulation are present in conjugated form.

Conjugated isoflavones undergo enterohepatic circulation, and on return to the intestine, they are deconjugated by bacteria with $\beta$-glucuronidase or arylsulfatase activity. The metabolites may be reabsorbed or further metabolized by gut microflora. Isoflavones can undergo further biotransformation that ultimately leads to the formation of $6^{\prime}$-hydroxy- $O$-demethylangolensin from genistein and $O$-demethylangolensin from daidzein (Joannou et al., 1995; Kurzer and Xu, 1997; UK Committee on Toxicity, 2003). The metabolic profile varies among individuals, with some individuals producing little or no $O$-demethylangolensin or equol, an intermediate metabolite of daidzein that is biologically active.

Limited ability of infants to produce equol was shown by Setchell et al. (1997, 1998), who detected equol in plasma of four of seven infants fed soy formulas. Equol was detected in cowmilk samples from Australia (King et al., 1998). In a study of children who had consumed soy or cow-milk formulas, Hoey et al. (2004) reported that genistein, daidzein, and glycitein and their metabolites were present in the urine of all seven soy formulafed infants in the 4-6-month age group, whereas $O$-demethylangolensin and equol were detected in $75 \%$ and $25 \%$ of soy formula-fed infants, respectively. In subjects aged 7 months to 7 years who were given a soy food challenge, $O$-demethylangolensin was present in the urine of $75 \%$ of soy food-fed subjects and $50 \%$ of control subjects, whereas total equol was present in the urine of $19 \%$ of soy foodfed subjects and $5 \%$ of control subjects.

Elimination half-lives in adults were reported at $\sim 4-9 \mathrm{hr}$ for genistein and its metabolites and 3-9 hr for daidzein and its metabolites after intake of $\leq 100 \mathrm{mg}$ or $\leq 2 \mathrm{mg} / \mathrm{kg}$ bw/day of each isoflavone as aglycones or glycosides (Lu et al., 1995; Setchell et al., 2001; Whitten and 
Patisaul, 2001; UK Committee on Toxicity, 2003). In volunteers who consumed formulations containing $70-100 \%$ unconjugated isoflavones at $1-16 \mathrm{mg} / \mathrm{kg}$ bw, mean half-lives were reportedly longer for total isoflavones $(\sim 6-13 \mathrm{hr}$ for genistein and metabolites and $4-16 \mathrm{hr}$ for daidzein and metabolites) compared to isoflavone aglycones $(\sim 2-7 \mathrm{hr}$ for genistein and daidzein) (Bloedon et al., 2002; Busby et al., 2002). [A value of $21.2 \mathrm{hr}$ for daidzein aglycone is an outlier and may be due to differences in experimental design and dose levels.] In subjects ingesting soy milk, urinary excretion peaked at $8-10 \mathrm{hr}$ and $95 \%$ of excretion occurred within $24 \mathrm{hr}$; total urinary excretion consisted of $1 \%$ aglycones and $99 \%$ glucuronidated metabolites (Lu and Anderson, 1998). One study showed that percent excretion of genistein and daidzein and their metabolites was not affected at total isoflavone levels of $0.7,1.3$, or 2.0 $\mathrm{mg} / \mathrm{kg}$ bw/day administered through soy milk powder (Xu et al., 1994). [The low recovery noted in this study reduces the Expert Panel's confidence in these results.]

It has been reported that urinary levels of daidzein and genistein and their metabolites are slightly lower in infants than in adults fed equivalent amounts of total isoflavones. This difference could possibly indicate slower renal clearance in early life (Setchell et al., 1998). In a study measuring urinary excretion of genistein and daidzein and their metabolites in four infants fed soy formula, average concentrations of total isoflavones in urine were total daidzein $2.9 \pm 0.3 \mathrm{mg} / \mathrm{L}$ and total genistein $1.5 \pm 0.2 \mathrm{mg} / \mathrm{L}$ (Irvine et al., 1998b). Mean percentages of daily total isoflavone intake detected in urine and normalized for creatinine levels were total daidzein $38 \pm 4 \%$ and total genistein $13 \pm 3 \%$. Total isoflavone excretion rates varied little between infants and ranged from $0.37 \pm 0.03$ to $0.58 \pm 0.06 \mathrm{mg} / \mathrm{kg}$ bw/day for daidzein and its metabolites and from $0.15 \pm 0.03$ to $0.32 \pm 0.04 \mathrm{mg} / \mathrm{kg}$ bw/day for genistein and its metabolites. Percent isoflavone excretion and excretion rate were not affected by age.

2.6.1.2. Experimental animals: As noted by the detection of isoflavones in blood of experimental animals fed soy-containing feed, isoflavones are absorbed after oral exposure (Chang and Doerge, 2000; Brown and Setchell, 2001; Lund et al., 2001a; Weber et al., 2001a).

Studies in rats showed the distribution of isoflavones and metabolites to fetuses during pregnancy or pups during lactation after ingestion of soy-containing feed by the dam (Brown and Setchell, 2001; Weber et al., 2001a). In one study, GD 20.5 fetuses and PND 3.5 pups contained about half the total genistein concentration and about $10 \%$ the total equol concentration measured in dams; total daidzein concentrations were about one-third lower in GD 20.5 fetuses and about the same in PND 3.5 pups compared to dams (Weber et al., 2001a). A second study reported that in pups born to dams fed a soy-containing diet, total genistein levels remained steady between birth and PND 12, whereas total daidzein levels were reduced by 50\% during the same time period (Brown and Setchell, 2001). On PND 16, the time when pups likely began eating feed, there was a 5- to 10-fold drop in total genistein and daidzein concentrations compared to earlier time periods. In contrast, total equol levels in pups decreased steadily after birth.

Studies in adult rats and mice fed soy-containing feed reported that blood total equol levels were 2-10 times higher than blood total genistein and daidzein levels (Brown and Setchell, 2001; Lund et al., 2001a; Weber et al., 2001a). In most cases, total equol levels were lower than or within the same order of magnitude as genistein and daidzein levels in fetuses or pups of rat dams fed soy-based diets (Brown and Setchell, 2001; Weber et al., 2001a).

The half-life for genistein and its metabolites after ingestion of soy products by rats was reported at $8.8 \mathrm{hr}$ (Table 16). No data were found for half-life of daidzein and its metabolites after ingestion through soy foods. Urinary levels of total genistein and daidzein were reported to be $\sim 25 \%$ of total equol levels in mice and $\sim 50 \%$ of total equol levels in rats. 
2.6.2 General toxicology-The FDA (1999) and the UK Committee on Toxicity (2003) reviewed numerous human studies examining the effects of soy foods on cholesterol levels. Based on well-conducted human studies, both the FDA and UK Committee on Toxicity concluded that consumption of soy as part of a diet low in saturated fat and cholesterol can lower total blood cholesterol and LDL levels.

In women receiving soy diets or supplements, there were no estrogenic effects on vaginal cytology in three studies (Baird et al., 1995; Murkies et al., 1995; Duncan et al., 1999b). A fourth study that provided very limited details and alternating exposure to non-soy based phytoestrogens reported increased vaginal cell maturation after women received soy flour supplements for 2 weeks (Wilcox et al., 1990). A fifth study with a longer exposure period (5 years) showed estrogenic effects on the endometrium (Unfer et al., 2004), but the study was limited by the inability to obtain endometrial samples in $20-25 \%$ of subjects during each of three examination periods (Foth and Nawroth, 2005). A sixth study reported in a preliminary study that there was increased proliferation of breast lobular epithelium and progesterone receptor expression in women who ate bread rolls containing $60 \mathrm{~g}$ soy product as textured vegetable protein (McMichael-Phillips et al., 1998). The final report (Hargreaves et al., 1999) with the full cohort of 84 individuals (including 33 subjects added from a tissue bank) showed no differences between the control group and the group eating soy rolls other than plasma isoflavone levels.

A study in immature rats examining uterotropic effects of infant formulas reported estrogenic effects for both soy and cow-milk formulas (Ashby et al., 2000b). Both cow-milk and soy formulas increased uterine weight independent of phytoestrogen content, with greater responses generally noted with soy compared to cow-milk formula when both were used at the concentration recommended for infant feeding. No evidence of estrogenic effects was observed in studies in which rats or monkeys were fed soy-based diets (Cline et al., 1996; Tansey et al., 1998; Ashby et al., 2000a; Wood et al., 2004); however, estrogenic effects occurred when soy isoflavones were added to a soy-free diet (Wade et al., 2003). A study in immature female mice found a soy-based diet to increase relative uterine weight and to antagonize the weight increase associated with feeding diethylstilbestrol, thus demonstrating weak estrogen agonist and antagonist properties (Mäkelä et al., 1995b). In a study in which rats were fed soy protein with and without the addition of conjugated equine estrogens, there were no additive effects between soy diets and equine estrogens (Tansey et al., 1998). Soy diets that were not alcohol-extracted to remove isoflavones antagonized the effects of estrogens on increased lactoferrin staining and epithelial luminal cell height. In some studies, increases in rodent uterine weight were greater in rats fed soy-free versus soy-containing diets (Thigpen et al., 1987, 1999; Ashby et al., 2000a,b).

2.6.3 Genetic toxicity—No genotoxicity studies were identified for soy formula or other soy foods.

2.6.4 Carcinogenicity-Because Asian populations have lower rates of some types of cancer, a possible role of soy products in protecting against carcinogenicity has been examined in reviews. Components of soybeans that were suggested as having possible chemopreventive properties included genistein, protease inhibitors, phytic acid, and $\beta$-sitosterol (Adlercreutz, 2002).

The following conclusions regarding soy intake and cancer were made by the UK Committee on Toxicity (2003):

- There was no convincing evidence that soy products have beneficial or adverse effects on development of breast cancer, 
- Evidence of protective effects against prostate cancer is greater in experimental animal than in humans, and

- Soy products do not seem to offer protection against colon cancer.

Possible mechanism suggested for protection against cancer have included changes in ratios of estrogen compounds (Xu et al., 1998; Lu et al., 2000b), inhibited bioactivation of procarcinogens (Rowlands et al., 2001), and antioxidant activity (UK Committee on Toxicity, 2003).

2.6.5 Potentially sensitive sub-populations-As noted in the Section 2.6.1, most genistein is present in the circulation as glucuronide conjugates. Human infants may have decreased ability to glucuronidate isoflavones because UDPGT activity is low in the fetus and neonate but gradually increases to adult levels in the first months to years of life (Doerge et al., 2002).

Sex-specific differences in metabolism of isoflavones in soy milk were reported (Lu and Anderson, 1998). Compared to men, women excreted higher percentages of daidzein, experienced a progressive decrease in percent urinary recovery and shortened half-life of isoflavones, and had longer elimination half-lives ( $\sim .4 \mathrm{hr}$ for daidzein and $6.7 \mathrm{hr}$ for genistein in women versus $\sim 2.9 \mathrm{hr}$ for daidzein and $3.8 \mathrm{hr}$ for genistein in men). One of six men and one of six women were capable of producing equol (16-28\% of daidzein intake) at the start of the study. Two weeks into the study, three additional women, but no additional men, developed the ability to produce equol.

Production of equol was found to vary greatly among individuals, and it is estimated that only $30-40 \%$ of the human population is capable of producing equol, an estrogenic metabolite of daidzein (Setchell et al., 1984; Kurzer and Xu, 1997; Xu et al., 1998; Rowland et al., 2000; UK Committee on Toxicity, 2003). Setchell et al. (2002) reviewed the role of metabolism of daidzein or for-mononetin (a clover isoflavone) to equol in experimental animal and human intestine and argued that much of the estrogenic activity of dietary soy products was due to the equol. They indicated, for example, that the menstrual cycle study of Cassidy et al. (1994), reviewed in Section 4.1, showed the greatest effect of a soy diet on menstrual cycle lengthening in the two women whose urinary equol excretion was high. The variability in human equol excretion after soy product intake has been attributed to variations in intestinal microflora, although the relevant microorganisms have not been identified (Rowland et al., 2000). As discussed above, Setchell et al. $(1997,1998)$ reported that infants fed soy formula excreted little, if any, equol, presumably due to immaturity of their bowel flora. The rat, by contrast, was characterized as an "equol-producing machine" because of its large cecum and abundant microflora (Setchell et al., 2002). Thus, susceptibility to the estrogenic effects of dietary soy products may vary from one individual to the next, may be low in infants fed soy formula, and may not be accurately modeled by rats.

\subsection{DEVELOPMENTAL TOXICITY DATA}

\subsection{Human Data}

3.1.1 Pregnancy exposure-North and Golding (2000), supported by the U.K. Medical Research Council, the U.K. Department of Health, the U.K. Department of the Environment, the U.K. Ministry of Agriculture, Fisheries, and Food, the BBC, Nutricia, Nestlé, "and other companies," carried out a case-control study of hypospadias as part of the Avon Longitudinal Study of Pregnancy and Childhood. This population-based project involved women with estimated delivery dates in 1991 and 1992. About $85 \%$ of the eligible population enrolled. Women completed questionnaires during pregnancy at 8,18 , and 32 weeks and at various times after birth. The 18- and 32-week pregnancy questionnaires included items identifying illness 
and drug exposures, and the 32-week questionnaire included dietary items. Cases of hypospadias $(n=51)$ were identified based on questionnaire responses in the first 3 years after birth, as well as "birth notifications," neonatologist records, and postmortem records. There were 7877 boys without hypospadias in the Avon database. Between approximately 5700 and 6600 of the pregnancies giving rise to these boys without hypospadias were used for various comparisons. Initial comparisons were made using the $\chi^{2}$ test followed by unadjusted logistic regression and then multivariate logistic regression analysis.

The univariate analyses showed a significant association between hypospadias and the use of iron supplementation during the first 18 weeks of pregnancy (odds ratio $[\mathrm{OR}]=1.87,95 \%$ confidence interval $[\mathrm{CI}]=1.02-3.46)$, vegetarianism during pregnancy $(\mathrm{OR}=3.53,95 \% \mathrm{CI}=$ 51.56-7.98), and influenza during the first trimester $(\mathrm{OR}=3.08,95 \% \mathrm{CI}=1.46-6.48)$. Specific questions about use of soy milk or imitation meat did not show significant associations with hypospadias in the offspring, but the number of exposed cases was small (two case children whose mothers used soy milk and five case children whose mothers used imitation meat). The use of "pulses" (peas, beans, lentils, chickpeas) four or more times/week was associated with hypospadias ( $\mathrm{OR}=7.56,95 \% \mathrm{CI}=2.25-25.42)$, based on three affected children. In the multivariate model considering iron supplementation and vegetarianism separately, the association of hypospadias with maternal vegetarianism persisted $(\mathrm{OR}=3.88,95 \% \mathrm{CI}=1.69$ 8.92); the association between hypospadias and iron supplementation was no longer statistically significant $(\mathrm{OR}=1.81,95 \% \mathrm{CI}=0.92-3.55)$. Influenza exposure remained associated significantly with hypospadias $(\mathrm{OR}=3.24,95 \% \mathrm{CI}=1.52-6.88)$. In a multivariate model combining iron supplementation and diet, using omnivorous diet without iron supplementation as the reference group, the association between vegetarianism and hypospadias was statistically significant $(\mathrm{OR}=4.99,95 \% \mathrm{CI}=2.10-11.88)$. The authors concluded that the association between maternal vegetarianism and hypospadias may be due to estrogenic constituents of soy and other plant products. As an alternative explanation, they proposed greater exposure of vegetarian women to pesticides used on food plants.

Strengths/Weaknesses: This population-based, relatively large study had a high participation rate. The prospective study design with information on potential confounders, the multiple sources of information for complete ascertainment of outcome, and the multivariate analysis were strengths. Dietary information was collected prospectively but only by interview and only at 32 weeks gestation. The rarity of the defect created sample size problems, and the information used for classifying outcome varied in consistency and reliability. There were no biological measures of exposure and the prevalence of exposure to soy foods was low in this sample. Possible confounding by pesticide exposure is an additional weakness.

Utility (Adequacy) for CERHR Evaluation Process: This study is not useful for the evaluation process.

\subsubsection{Exposure of infants}

3.1.2.1 Growth and nutrition: Reports on the ability of soy formula to support normal growth and to provide adequate nutrition are presented. Case series are presented first followed by controlled studies. Although several of these reports involved premature infants, soy formula is not recommended currently for premature infants.

Kulkarni et al. (1980), funding not indicated, reported 22 cases of rickets in very low birthweight $(<1500 \mathrm{~g})$ infants fed a soy-isolate formula. The soy formula contained $700 \mathrm{mg} / \mathrm{L}$ calcium, $500 \mathrm{mg} / \mathrm{L}$ phosphorus, and $400 \mathrm{IU} / \mathrm{L}$ vitamin $\mathrm{D}$. When feeding was tolerated, the formula was supplemented by a multivitamin; additional calcium lactate or vitamin $\mathrm{D}$ were also provided depending on neonatologist preference. The mean daily intake of each of these 
supplements was measured during infant hospitalization periods. The lowest mean serum phosphorus concentration occurred at 7-10 weeks, which preceded the active phase of rickets by about 2 weeks. The highest serum levels of alkaline phosphatase occurred at 11-14 weeks, 2 weeks before the healing phase. The lack of a control group prevented the authors from drawing conclusions from the data, and no statistical analyses were carried out. However, the authors suggested further studies investigating calcium, phosphorus, and vitamin D in very low birth-weight infants with rickets, in particular those fed soy formula. This report led to a controlled prospective study (Kulkarni et al., 1984), which is discussed below.

Strengths/Weaknesses: This case series involved a special population of very low birth-weight infants.

Utility (Adequacy) for CERHR Evaluation Process: Although this report may be useful for hypothesis-generation, it is not useful in the CERHR evaluation process.

Fomon (1959), supported by Mead Johnson and Ross Laboratories, evaluated growth and nitrogen retention in four infants aged 4-6.5 months receiving a soy formula with $6.8 \%$ caloric protein. The author reported that weight gain was normal in these infants, and nitrogen retention was at least as great as that estimated in three of the infants during a previous month during which they consumed human milk.

Strengths/Weaknesses: This study involved a small sample $(n=4)$ with no comparison group.

Utility (Adequacy) for CERHR Evaluation Process: This study is not useful in the CERHR evaluation process.

Sellars et al. (1971), supported by Borden, Inc., reported length and weight measurements for 1583 Caucasian infants during the first year of life. Mothers were encouraged to breast feed as long as possible. Infants not being breast-fed, or being weaned from breast feeding, were assigned to cow-milk or soy formula based on family history of allergy [although the assignment strategy was not discussed]. The comparison groups for evaluation of height and weight included 401 breast-fed infants, 239 infants on soy formula, and 839 infants on cowmilk formula. Infants were evaluated monthly until the age of 6 months and bi-monthly thereafter. Height and weight were plotted on growth curves, and statistical comparisons were made by an unspecified method. No differences in growth were detected between the three feeding groups. The authors concluded that any of the three feeding methods would support normal growth.

Strengths/Weaknesses: The large sample, repeated measurements of weight and lengths, and 1-year follow up are strengths. The study was limited to one ethnic group, and there may have been selection bias in mothers who did not breast feed or who discontinued breast feeding, as well as in assignment to soy formula group on the basis of family history of allergy. Head circumference was not evaluated. Growth curves should have differed between breast-fed and formula-fed infants at least before introduction of solid foods; so, it is unclear how statistical comparisons (whatever they were) could have shown no differences. There were apparently no adjustments for potential confounders and baseline differences between groups. The introduction of solid food by pediatricians at 1 month is another weakness.

Utility (Adequacy) for CERHR Evaluation Process: This study is of minimal utility in the evaluation process.

Kay et al. (1960), supported by the Borden Company, followed normal, healthy infants fed soy formula (Mull-Soy ${ }^{\circledR}$ ) or evaporated cow-milk formula (Silver Cow $\left.{ }^{\circledR}\right)$ to evaluate 
differences in weight, length, hemoglobin, hematocrit, and plasma biochemical values. [An additional 32 infants on unspecified feeding methods were evaluated at birth or at 3 months of age and are not considered here.] At $24 \mathrm{hr}$ of age, 14 infants were started on soy formula and 14 were started on evaporated cow-milk formula and followed for 3 months. Efforts were made to match patients for sex, ethnic origin, and birth weight. Infant weight and length were measured at clinical visits every 2 weeks. Hemoglobin, hematocrit, and plasma biochemical values were measured from blood drawn shortly after birth and at 3 months of age. Plasma protein electrophoresis was carried out to quantify albumin, $\alpha 1$-globulin, $\alpha 2-$ globulin, $\beta$-globulin, and $\gamma$-globulin.

Growth increases in the soy formula and evaporated milk formula were similar and paralleled normal growth curves (based on Iowa Growth Charts). No statistically significant feeding group difference were identified in hemoglobin, hematocrit, or plasma biochemistry values except as shown in Table 21. The study authors concluded that nutritive value and infant growth measurements were similar in infants fed soy formula and evaporated cow-milk formula from birth to 3 months of age.

Strengths/Weaknesses: Some effort was made to match groups at the outset on important potential confounders. The use of blood measures at two time points is a strength. Although there was a control group, diet was unrestricted and there was no comparison to breast-fed infants. It was unclear if infants were exclusively formula-fed for the duration of study and how they were selected for feeding groups. The small sample size provided limited power for the conclusion of no differences in growth curves, and there was no measurement of head circumference. Only about half of the already small sample size were available for the full set of biochemical measures at 3 months of age. Intake was not reported, and it is like that the evaporated milk group would have had down-regulation of intake. Without knowing whether feedings were isocaloric, the groups cannot be meaningfully compared. The differences in total protein $\alpha 2$-globulin levels are small and not clinically significant.

Utility (Adequacy) for CERHR Evaluation Process: This study is useful in the evaluation process.

Cherry et al. (1968), supported by Gerber Products Co., tested the acceptance, tolerability, and nutritional value of a new (in 1968) soy formula provided to healthy newborn infants. When discharged from the hospital, mothers were given a 3-month supply of the soy formula or a marketed cow-milk formula (Modilac ${ }^{\circledR}$ ). Formulas were packaged in identical containers. The soy formula contained isolated soy protein $(15.1 \%)$, soy oil (37.5\%), and carbohydrate ( $47.4 \%$ as sucrose and corn syrup solids). [The Expert Panel notes that this soy formula contains more fat and less carbohydrate than soy formulas in current use. As noted in Section 1.1, current soy formulas include soy protein isolate (14.6-18\%), vegetable oils (21-28.6\%), and carbohydrate as corn syrup (40.8-55\%) and sugar (10.2-11\%).]

Formulas were approximately isocaloric. Mothers recorded the amount of formula consumed at each feeding, stool characteristics (color, consistency, and frequency), vomiting, acceptability of the feeding, and satiety after the feeding. Clinic visits included standard wellbaby physical examinations, immunizations, measurements (weight, length, and head circumference), and blood work. Other procedures included muscle reflex patterns (twice in 6 months), $x$-rays of the tibia (at 6-8 weeks and again at 4-6 months), and more extensive blood work (at 2, 4, and 6 months). A reference population for growth measurements consisted of 200 children of similar socioeconomic and racial background chosen from the Collaborative Child Development Project at Charity Hospital in New Orleans. Fifty-eight of the 73 infants initially enrolled completed 6 months of follow-up. Proportions of infants with symptoms were compared by $\chi^{2}$ testing, and growth parameters were compared using $t$-tests. Multiple 
regression was used to evaluate the relationship between nutritional components of the formulas and weight gain and ANCOVA was used to evaluate growth data and food intake.

Growth data are illustrated in Figure 4. Soy formulafed infants, especially girls, lagged behind cow milk-fed infants with significant differences primarily between 2 and 4 months of age. The reference population growth parameters (not shown) were generally between those of the cow-milk and soy groups. Formula intake level averaged 2 oz/day more in the cow-milk group than in the soy group, resulting in a 10\% difference over the initial 56 days. The cow-milk group gained significantly more weight per oz of formula than the soy group. Cow milk-fed infants had greater intakes of methionine [which today is added as a supplement to soy formulas], calcium, and folic acid, and soy formula-fed infants had greater intakes of iodine, thiamine, vitamin $\mathrm{C}$, and vitamin $\mathrm{B}_{12}$. Maternal observations showed more vomiting in the milk-fed group (21\% slight, $5 \%$ frank) compared to the soy formula-fed group (13\% slight, $1 \%$ frank, $P=0.001$ ). Eighteen percent of the infants in the milk-fed group were categorized as hungry after feeding compared to $7 \%$ in the soy formula-fed group $(P=0.001)$. Loose stools were more frequently reported in the soy formula-fed group (27\%) than the milk-fed group $(19 \%, P=0.001)$. There were no instances of anemia, and hematologic parameters did not differ consistently by feeding group.

The study authors concluded that the slower growth in soy formula-fed girls might be of concern.

Strengths/Weaknesses: Strengths including the blinding of participants to feeding-group assignment, measurements of head circumference and blood parameters, use of x-rays, an additional outside reference population, and assessment of differences in growth by infant sex. It is a strength that there was specific recording of the amounts of formula consumed at each feeding, allowing for recognition that the cow-milk group consumed more formula; however, there is no way to know if the accuracy of recording in the two groups differed. There could have been selection bias in mothers who elected not to breast feed and therefore could participate. The drop-out rate was high, with 15 of 73 subjects not completing the 6 months' evaluation. The sample was very small, providing limited power, especially for stratified analysis on infant sex. There was no comparison to exclusively breast-fed infants, and the introduction of solids was not specified. A repeated-measures type of analysis would have been appropriate for growth over time.

Utility (Adequacy) for CERHR Evaluation Process: This study is marginally useful for the evaluation process, limited by concerns about accuracy of intake recording and differences in modern soy formula from the product used at the time of the study.

Dean (1973), supported by Ross Laboratories, evaluated growth and development over the first 6 months of life in children fed a commercial soy formula or a commercial cow-milk formula, or who were breast-fed. Babies whose mothers chose to breast feed were enrolled into the breast-fed group. There were 26 such babies at the beginning of the study, but only six remained on breast feeding by 6 months of age. Among babies whose mothers chose to bottlefeed, 26 were assigned to soy formula (of whom 24 completed the 6-month trial) and 29 were assigned to cow-milk formula. There were 35 children in the cow-milk group at the end of the study because some breast-fed children who "failed" breast feeding within the first month of life were reassigned to the cow-milk formula group. [The method of assignment to soy or cow-milk formula was not discussed.] All babies were at least $2800 \mathrm{~g}$ at birth and were normal on examination by the investigator. Solid foods were added at 6-12 weeks of age. Weight, length, and head circumference were measured monthly. The Denver Developmental Screening test was administered [how often and by whom is not indicated]. Blood was obtained at different visits in different children for chemistry and hematology evaluation. 
The only abnormality noted in the children on soy formula was perianal redness that responded to zinc and cod-liver oil cream. The 24 children in this group who finished the study followed their centile growth curves during the study period. There was more disparity in growth in the cow-milk formula group, with 5 of 39 children having weight percentiles that exceeded their length percentiles. [The number of children (39) in the study Results section is different from the number (35) in the study Methods section and from the number (40) in one of the study data tables.] All infants were said to be "neurologically normal or advanced" except for one child in each of the three feeding groups. The laboratory values were normal in all children, but the blood urea nitrogen was highest in the cow-milk formula group, intermediate in the soy formula group, and lowest in the breast-fed group, proportional to the nitrogen content of the formulas/milk (Fig. 5). The author concluded that soy formula was an adequate nutrition source.

Strengths/Weaknesses: Strengths include the use of two formula-fed groups and a breast-fed comparison group, repeated measurements of growth including head circumference, and developmental assessments. Despite these strengths, the sample size was very small, and dropouts could have influenced the results. It seems inappropriate for infants who "failed" breast feeding to have been included in the cow-milk group. There could have been selection bias in mothers' assignment to feeding groups. The introduction of solid foods was not taken into account, and there was no report of how many calories were given to the groups. A repeated-measures analysis would have been useful.

Utility (Adequacy) for CERHR Evaluation Process: This study is of limited utility.

Jung and Carr (1977), support not indicated, studied the nutritional and growth differences between 20 infants on soy formula (Isomil $®$ ) and 20 infants on cow-milk formula (Similac ${ }^{\circledR}$ with Iron). Healthy infants were assigned by gestational age, weight, race, and sex to receive one of the formulas for 16 weeks. Cereal was introduced at 4 weeks, and vegetables were introduced at 8 weeks. Infants were assessed for formula tolerance, weight, length, and head circumference. The researchers reported that general acceptance of both formulas was good, and no infants needed formula changes. All growth measurements were normal, and no significant differences in body weight, length, or head circumference were observed between the groups. Hemoglobin, hematocrit, protein, albumin, calcium, phosphorous, glucose, urea nitrogen, and alkaline phosphorous were also within the normal range and did not significantly differ between groups. The authors conclude that the soy formula used in this study imposed no significant nutritional differences compared to cow-milk formula.

Strengths/Weaknesses: There was some attention to prospective matching of infants on important confounders. The introduction of specified solids at specified times in both groups is a strength, as is the use of blood measures and measurement of head circumference. The sample size was small, however, with limited power to detect differences between groups. There was inadequate adjustment for potential confounders, and there was no breast-fed comparison group.

Utility (Adequacy) for CERHR Evaluation Process: This study is of limited utility.

Köhler et al. (1984), support not indicated, reported the growth and food intake of 59 infants aged 6-25 months on human milk, cow-milk formula, or soy formula. Participants were selected by 6 weeks of age from two well-baby clinics, one of which provided infants with cow milk-based formula (Similac ${ }^{\circledR} ; \mathrm{n}=20$ ), and the other of which provided infants on soy formula (Isomil@; $n=13)$. The remaining children $(n=26)$ were fully breast-fed. Mothers recorded 48 -hr intake at $6,14,22$, and 26 weeks. Cow-milk and soy formula consumption was measured by administering a standard formula volume and weighing the amount remaining 
after feeding. Breast-fed infants were weighed by their mothers before and after feedings. There were no significant differences in consumption between formula groups, but breast-fed infants consumed significantly less per $24 \mathrm{hr}$ than the formula-fed infants. There were no statistically significant differences in energy intake between infants on soy and cow-milk formulas. The difference in intake between breast-fed and all artificially fed infants was statistically significant at 6 and 14 weeks [statistical methods not indicated].

Growth was measured by weight, length, and skinfold thickness and followed for 52 weeks. Infants on soy formula gained less weight in the first 6 weeks than breast-fed infants and those on cow-milk formula. The authors suggested that this difference might be explained by infants in the soy group being significantly heavier by an average of $200 \mathrm{~g}$ at birth. After 6 weeks of age, no significant differences in mean weight were detected between the three groups. No significant differences were detected between the three groups in length or sum of skin-fold thicknesses.

Radiographs of the left hand were taken at 3 and 6 months of age to measure bone mineralization and maturation. The internal $(d)$ and external $(D)$ diameters of the narrowest part of the diaphysis of the second metacarpal bone were used to "represent" the cortical crosssectional area as $D^{2}-d^{2}$. [The Expert Panel notes that the cross-sectional areas would be $\pi$ $\left(D^{2}-d^{2}\right) / 4$.] This measurement, which the authors cite as representative of bone ash content per unit length, was used to indicate bone mineralization. Ossification centers were measured to characterize skeletal maturation. At 3 months of age, the soy formula group showed slower mineralization and bone maturation than both the breast-fed group and the cow-milk group; however, by 6 months these differences in skeletal development were no longer statistically significant.

The authors concluded that although they believed birth weight differences could explain differences in weight gain during the first 6 weeks of life, they could not exclude the possibility that nutrients were less well absorbed from soy formula than from human or cow milk.

Strengths/Weaknesses: It is a strength that this study compared three feeding groups with recording of infant intake and measures of bone mineralization. Multivariate analysis should have been used to adjust for differences such as birth weight between groups. There could have been selection bias among mothers who elect to breast feed or not. Assignment to formula feeding groups was by clinic location and not within clinics, and there could have been differences between women associated with a site that were not taken into account. There was 1-year growth follow-up, but no head circumference measures were taken, and there was no mention of adjusting for supplementary foods, including milk in the breast-fed group. The sample size was small with inadequate adjustment for other potential confounders.

Utility (Adequacy) for CERHR Evaluation Process: This study is of marginal utility.

Steichen and Tsang (1987), supported by the National Institute of Child Health and Human Development (NICHD), the Children's Hospital Research Foundation, the Veteran's

Administration of Cincinnati, the Jewish Hospital of Cincinnati, Ross Laboratories, and Gerber Laboratories, carried out a prospective, randomized, double-blind, longitudinal study over 1 year to determine differences in growth and skeletal mineralization between infants fed soy formula and those fed cow-milk formula. Subjects were healthy term infants appropriate for gestational age and born to healthy, middle-class white mothers. Infants were given a soy formula (Isomil@ with Iron, $\mathrm{n}=18$ ) or a cow-milk formula (Similac ${ }^{\circledR} 20$ with Iron, $\mathrm{n}=17$ ). Infants were fed exclusively with the assigned formula from birth until 6 months of age, when baby food was provided by investigators and introduced to the diet. At 6 weeks and 3, 6, 9, and 12 months, measurements were made of weight, length, head circumference, and bone 
mineral content by dual photon absorptiometry of the midshaft and wrist of the left radius and ulna. Student $t$-test and ANOVA with Duncan multiple range test were used in the comparisons.

There were no significant differences at the start of the study in weight, length, or head circumference. Energy intake during the study was the same in both groups, and no significant differences were detected between the groups in weight, length, head circumference, or the rates at which these measurements increased over the study period. All infants were within the normal growth range based on the National Center for Health Statistics growth curves. The ratio of bone mineral content to bone width was significantly lower in the soy formula group at $3,6,9$, and 12 months of age. Both groups showed a decrease in bone mineral content per bone width until 26 weeks of age and then a subsequent increase until 52 weeks. In the infants on cow-milk formula, bone mineral content rebounded to initial levels, but in the soy formulafed infants, bone mineral content remained significantly lower than initial values.

The authors noted that the lower bone mineral content of the soy formula-fed infants may not have been clinically significant and was similar to that of breastfed infants measured at 3,6, and 12 months of age in a previous study at the same center. They further suggested that cowmilk formula may cause bone to be hypermineralized, rather than soy formula causing belownormal bone mineralization. Although the authors did not analyze whether there was benefit or harm due to increased bone mineral content, they noted that in rats, bone hypermineralization early in life was found to decrease long-term mineral retention, and they questioned a relationship to osteoporosis. The authors also speculated that the lower bone mineral content in soy formula-fed infants could have been due to decreased availability of calcium and protein.

Strengths/Weaknesses: The prospective, randomized, double-blind design is a strength. There was 1-year follow-up with multiple measures, including head circumference and bone mineral content. Use of a homogeneous racial and socioeconomic group is a strength, although it limits generalizability of the study. Solid food was introduced at the same time and food was provided by investigators, and there were similar proportions of subjects in the formula groups receiving solid food. Weaknesses include the lack of a concurrent breast-fed comparison group and the small sample size. There could have been selection bias in women who elected to formula feed, and there was no adjustment for potential confounders.

Utility (Adequacy) for CERHR Evaluation Process: This study is of limited utility, except for the bone mineral density portion of the study, which may be of particular utility, despite very small sample size.

Hillman (1988) and Hillman et al. (1988), supported by Mead Johnson Co., studied 31 term infants receiving either human milk $(\mathrm{n}=9)$, the cow-milk formula Enfamil ${ }^{\circledR}(\mathrm{n}=11)$, or the soy formula ProSobee ${ }^{\circledR}(\mathrm{n}=11)$. The decision to breast- or formula-feed was made by each infant's mother. Within the formula group, babies were placed on soy formula by their private pediatricians for "colic or spitting up." Cereal was allowed at 4 months of age, and other solid foods at 6 months. Breast-fed infants were given vitamin D 400 IU/day as a supplement (both formulas also included this level of vitamin D). The infants were examined at entry into the study (age $=<3$ weeks) and then at 2, 4, 6, 9, and 12 months. Samples were taken for measurement of serum vitamin $\mathrm{D}, 25$-hydroxyvitamin $\mathrm{D}, 1,25$-dihydroxyvitamin $\mathrm{D}$, parathyroid hormone, alkaline phosphatase, calcium, phosphorus, magnesium, and albumin. One urine sample was also taken randomly from each subject for measurement of calcium, phosphorus, magnesium, and creatine. Weight, body length, head circumference, bone width, and bone density (single-photon absorptiometry, mid-humerus) were measured at each visit. Trends in data over time were analyzed by linear regression, and mean slopes were analyzed using the 2-tailed Dunnett's $t$-test. ANOVA was carried out for 6-month values, and differences were identified by post-hoc Duncan test. 
No significant differences by feeding type were detected in the rate of increase of bone width, bone mineral content, or bone mineral content as a function of body length. Serum and urine measurements are shown in Table 22. The differences noted in vitamin and mineral levels were considered to reflect homeostatic adjustments to differing mineral levels in the food sources. The authors concluded that infant bone mineralization was not affected by food type when adequate vitamin $\mathrm{D}$ and mineral intake were ensured.

Strengths/Weaknesses: The use of three feeding groups is a strength, as are the multiple measurements of growth, including measurements of head circumference and bone density and the blood and urine measures. The specification of times when solid foods were allowed is a strength, but it is a weakness that solid food was started early and there was no documentation of the dietary intakes of the three groups. Other weaknesses include the small sample size and the lack of adjustment for potential confounders. In addition, the soy group was selected for a specific indication, and these children could have been on cow-milk formula before enrollment (up to 3 weeks). Using "colic" as an indication for recommending soy infant formula introduces a potential bias in group assignment.

Utility (Adequacy) for CERHR Evaluation Process: This study is of limited utility, except for the bone mineral density portion of the study, which may be of particular utility, despite very small sample size.

Lasekan et al. (1999), supported by Ross Products Division, Abbott Laboratories, carried out a randomized, masked, parallel 1-year clinical feeding study to determine the effects of soy protein-based formulas and supplemental free nucleotides on the growth of infants. Healthy term infants were recruited from nine private pediatric practices. Infants were given a soy formula Isomil ${ }^{\circledR}, \mathrm{n}=73$ ), the soy formula with $72 \mathrm{mg} / \mathrm{L}$ supplemental free monomeric nucleotides $(n=73)$, or mixed feeding $(n=67)$ consisting of 2 months exclusive breast feeding followed by a cow-milk formula (Similac ${ }^{\circledR}$ with Iron supplementation), without nucleotide fortification. Assignment was randomized by sex in the two soy groups. Infants were enrolled in the mixed-feeding group if their mothers chose to breast feed. Children were allowed to eat solid foods after 4 months of age. Formula tolerance and growth measurements (weight, length, and head circumference) were measured at enrollment and at 0.5, 1, 2, 4, 6, 7, and 12 months of age. Growth data were analyzed by 2-way ANOVA with feeding and sex as interaction terms.

At birth, infants on soy formula were heavier and longer than infants on nucleotidesupplemented soy formula (mean \pm SEM weight: soy $3616 \pm 66$ compared to soy + nucleotides $3254 \pm 78 \mathrm{~g}$; length: soy $52 \pm 0.4$ compared to soy + nucleotides $50 \pm 0.4 \mathrm{~cm}$ ). There was a significantly larger weight gain between 2 weeks and 1 month of age in the mixed-feeding group (mean \pm SEM: $42.6 \pm 1.8 \mathrm{~g}$ /day) compared to infants fed unsupplemented soy formula $(34.1 \pm 1.3 \mathrm{~g} /$ day) or soy formula with supplemental nucleotides $(36.7 \pm 1.3 \mathrm{~g} /$ day). Between 2 and 4 months of age however, infants fed unsupplemented $(24.2 \pm 0.7 \mathrm{~g} /$ day $)$ and supplemented soy formula $(24.2 \pm 0.8 \mathrm{~g} /$ day) gained significantly more weight than mixed-feeding infants $(20.3 \pm 0.3 \mathrm{~g} /$ day). After 4 months of age, no significant weight gain differences were detected between the groups. Children in the unsupplemented soy group were significantly longer than mixed feeding infants at 7 and 12 months of age. Mean head circumference for infants on soy formula with supplemental nucleotides was larger than the mixed-feeding group at 6 months of age. Over the course of the entire study, no differences in length and head circumference measurements between groups were detected. All growth measurements were consistent with National Center for Health Statistics normal values.

Mean hemoglobin concentration, plasma albumin, and blood urea nitrogen were within the normal ranges in all groups. There was a significantly higher level of blood urea nitrogen in 
both soy groups than in the mixed-feeding group $(P<0.0001$, mean \pm SEM not reported). The authors noted that this finding was expected due to the typically higher levels of protein in soy formula than in human milk.

The study authors concluded that over the first year of life, growth of healthy term infants fed soy formula or soy formula with supplemental nucleotides was similar to infants on a mixedfeeding regimen of human milk followed by cow milk-based formula. They also noted that they could detect no significant effects of supplemental nucleotides on growth.

Strengths/Weaknesses: Strengths include the use of three defined feeding groups, the relatively large sample size, random assignment by sex to soy-feeding groups, the specified time for introduction of solid foods, and the multiple measures of growth including head circumference. The mixed-feeding was group defined by length of exclusive breast feeding followed by cow-milk formula. It is a weakness that solid food was started early. There may have been selection bias in mothers who elect to breast feed. Random assignment by sex to the two formula groups did not result in equality between the groups; with 73 children in each group, there was still a mean difference of almost $400 \mathrm{~g}$ in birth weight between the groups, suggesting that there was some other fundamental difference between the groups at baseline. There was inadequate adjustment for potential confounders, and a repeated-measures analysis would have been preferable.

Utility (Adequacy) for CERHR Evaluation Process: This study is of limited utility.

Callenbach et al. (1981), support not indicated, reviewed the records of 65 surviving infants who were born weighing $<1500 \mathrm{~g}$ and for whom radiologic and biochemical data were available. There were 37 infants with radiologic evidence of rickets and 28 infants without such evidence. Soy-isolate formulas had been given to 32 of the children with rickets and 26 of the infants without rickets $[\boldsymbol{P}=\mathbf{0 . 6 9}$, Fisher exact test $]$. The authors observed that these infants were very ill, and that long periods of parenteral feeding had been required during the initial weeks of life.

Strengths/Weaknesses: Strengths include the focus on rickets and the evaluation of a homogenous special population of very low birth-weight infants with radiologic findings. Weaknesses include the retrospective nature of the record review, the lack of adjustment for confounders, and the small amount of variability in exposure: it seems that 58 of 65 infants in the sample had been given soy formula.

Utility (Adequacy) for CERHR Evaluation Process: This study is not useful in the evaluation process.

Kulkarni et al. (1984), supported by Ross Laboratories, carried out a clinical trial in 46 very low birth-weight $(\leq 1500 \mathrm{~g})$ infants given one of three formulas to ascertain the relationship between formula type and rickets. Infants were randomly assigned to soy formula (Isomil@), cow-milk formula (Similac ${ }^{\circledR}$ with Iron), or a hypercaloric cow milk-based formula designed for low birth-weight infants (Similac® $24 \mathrm{LBW}$ ), and followed until 3-4 months of age. Formula compositions are given in Table 23.

Formula was introduced by continuous nasogastric drip until a weight of $1600 \mathrm{~g}$ was reached and infants could tolerate bolus feeding. Roentgenograms of one knee and one wrist were taken in all infants when they were between 8 and 17 weeks old. Infants who did not reach a daily alimentary intake of $80 \mathrm{kcal} / \mathrm{kg}$ by 5 weeks old were not included in the study. Initial numbers of infants in the soy formula, cow milk-based formula, and hypercaloric cow milk-based 
formula groups were 15,18 , and 13 , respectively; however, these numbers dropped to nine, eight, and eight.

Nutrient intake was analyzed by repeated measures ANOVA and the Scheffé method of multiple comparisons. No significant formula-group differences were detected in intake of calories, protein, phosphorus, or vitamin D. Calcium intake was significantly higher in the soy formula-fed infants than those fed cow milk-based formula. Blood chemistry data collected during hospitalization were analyzed using the Bonferroni multiple comparison test. Data collected after discharge were averaged for each infant, and the values were analyzed using ANOVA followed by the Duncan multiple range test.

No significant differences were found before 3 weeks of age. Infants on soy formula had significantly lower serum levels of phosphorus at Weeks 3, 5, and 8-14 and higher serum levels of alkaline phosphatase at Week 5 and Weeks 8-14 than infants in one or both of the cow-milk formula groups. Active rickets was diagnosed by x-ray based on the concurrence of two radiologists. Of 15 infants in the soy formula group, nine developed rickets. In contrast, only one case of rickets was diagnosed in the two cow-milk groups combined. The study authors speculated that the higher incidence of rickets in very low birth-weight infants fed soy formula may have been due to decreased absorption or solubility and bioavailability of minerals and discouraged the use of soy isolate formula for this population.

Strengths/Weaknesses: Strengths include the study of a homogeneous special population of very low birth-weight infants, random assignment to one of three formula groups, similarity of caloric, protein, phosphorus, and vitamin D intake between groups, follow-up to 3-4 months, specification of the mode of delivering formula, specification of exclusionary criteria, use of appropriate repeated-measures method of analysis, and the use of two radiologists to confirm the diagnosis of rickets. Weaknesses include the small sample size and high drop-out rate.

Utility (Adequacy) for CERHR Evaluation Process: This study is relevant to the risk for rickets in very low birth-weight infants, but the drop-outs and excluded infants could be important limitations on its utility.

Naude et al. (1979), support not indicated, conducted a 35-day trial in 40 preterm infants to compare the effects of feeding a cow-milk formula compared to a soy formula. Infants who weighed between 1500 and $1800 \mathrm{~g}$ at birth, seemed healthy, were appropriate for gestational age, and whose mothers had decided not to breast feed were assigned randomly to either a cowmilk or a soy formula feeding group. The children received formula amounts up to $180 \mathrm{~mL} /$ $\mathrm{kg}$ bw/day. All infants were given supplemental vitamins. Weights were taken on study Days 1 and 2 and then on alternate days for the duration of the study. Head circumference and body length were measured on Day 1 and then weekly. Blood samples were taken once between Days 7 and 10 and again between Days 32 and 35. Samples were analyzed for serum concentration of albumin, calcium, phosphorus, alkaline phosphatase, and sodium, and for blood urea nitrogen and hemoglobin. Student $t$-test was used for statistical analysis.

The researchers report that it became immediately evident that the infants in the soy formula group were not progressing well. Of the first 10 infants allocated to this group, seven were withdrawn because of weight loss or poor weight gain, and one died with necrotizing enterocolitis. Only two infants completed the 35-day trial. Only two of the first 10 infants in the cow-milk group were withdrawn, one due to jaundice and the other due to vomiting and a diaphragmatic hernia. The remaining eight were said to progress "satisfactorily." Due to the low success rate, the researchers changed the feeding regimen for the 20 subsequent subjects, increasing the daily intake from 180 to $200 \mathrm{~mL} / \mathrm{kg}$ bw/day. The remaining infants allocated to the soy-formula group progressed more satisfactorily with the increased intake, and only two 
were withdrawn due to jaundice. On the higher-volume feeds, infants on soy formula gained an average of $305 \mathrm{~g}$ less weight and acquired $1.6 \mathrm{~cm}$ less height than infants on cow-milk formula. [The data table shows a height difference of $16 \mathrm{~cm}$, which the Expert Panel assumes should be $16 \mathrm{~mm}$.] Serum albumin and phosphatase levels were lower in soy formulafed infants. The study authors recommended that caution be exercised in the feeding of soy formulas to preterm babies.

Strengths/Weaknesses: Strengths include the random assignment to soy or cow-milk formula, the multiple growth measurements including head circumference, the use of blood measures, and the use of a homogeneous special population sample of preterm, low birth-weight infants. Weaknesses include the small sample size and inability to adjust for potential confounders. In addition, the change in strategy mid-stream made it unclear whether reduced growth in subsequently enrolled soy formula-fed infants was due to a still-inadequate supply of formula.

Utility (Adequacy) for CERHR Evaluation Process: This study is somewhat useful in the evaluation process.

Shenai et al. (1981), supported by Ross Laboratories, prospectively studied nutritional retention over 28 days in 19 very low birth-weight $(<1530 \mathrm{~g})$ infants on either soy formula ( similar to Isomil@; $\mathrm{n}=10$ ) or cow milk-based formula (Similac ${ }^{\circledR}$ with Iron; $\mathrm{n}=9$ ). Infants with a gestational age of 31 weeks or less were recruited from the neonatal intensive care center of the University of Oregon Health Science Center. The very low birth-weight infants were of normal size for gestational age and did not have metabolic or congenital anomalies. Within 7 days of birth, infants were fed at 3-hr intervals with the assigned formula in addition to a multivitamin and vitamin E 25 IU/day. When the infant had achieved full oral intake of the formula for 8 days, a 96-hr metabolic balance study was carried out. Infants were weighed daily and occipitofrontal circumference and crown-heel length were measured weekly. Stool and urine samples were frozen until analysis for calcium, phosphorus, and nitrogen; stools were also analyzed for fat. Blood samples were taken at approximately 4, 14, 21, and 28 days postnatally. The two groups were studied successively [not randomized]; however, stool and urine analyses were concurrent. Statistical analysis was carried out by $t$-test and ANCOVA.

No formula-group differences in fat intake or absorption were detected. The soy formula-group had a significantly higher intake of nitrogen and calcium and lower intake of phosphorus than the cow-milk group. Because there were no detected significant differences in calcium excretion, absorption, or retention rates, the authors proposed that the absence of lactose in soy formula did not interfere with calcium retention or homeostasis. Although nitrogen intake was higher in the soy formula- than cow-milk formula-group, there was significantly lower nitrogen retention in the soy-formula group. Soy formula-fed infants had significantly lower phosphorus intake, absorption, and urinary excretion and a higher fecal excretion than cow-milk formulafed infants. However, no significant difference in overall phosphorus retention between groups was detected.

No significant differences were detected between groups at any time in serum calcium or alkaline phosphate concentrations. Mean serum phosphorus levels were lower in soy formulafed than cow milk-fed infants on PND 14, 21, and 28. No significant differences were found in mean serum total protein and albumin values at any time, and measurements were within the normal range for very low birth-weight infants. Mean blood urea nitrogen values were significantly higher in the soy-formula group on PND 14, 21, and 28 compared to the cowmilk group. Weight gain, crown-heel length, and occipitofrontal circumference did not significantly differ between the two feeding groups. 
The authors stated that the study period was too short for these measurements to be valuable in determining growth effects. The authors also noted that the study may not have shown effects of sustained soy-formula feeding due to the small sample size, short duration, and lack of longterm follow-up. They specifically questioned whether long-term use of soy formula in very low birth-weight infants would have led to phosphorous-deficiency rickets.

Strengths/Weaknesses: The use of a homogeneous, special population sample of very low birth-weight infants and the repeated measurements for growth including head circumference and blood samples are strengths; however, the follow-up period of $<1$ month was too short, and the small sample size gave very limited power to detect differences.

Utility (Adequacy) for CERHR Evaluation Process: This study is not useful in the evaluation process.

Hall et al. (1984), supported by Mead-Johnson Laboratories, conducted a randomized, controlled study to compare a calcium- and phosphorus-supplemented soy formula with a whey-predominant premature formula with regard to nutrition and growth in very low birthweight infants. Forty infants from 3-8 weeks of age who had birth weights $<1500 \mathrm{~g}$ and were not breastfed were recruited for the study. Formula assignment was made at the time of feeding initiation and all infants were given supplemental vitamins. Seventeen infants fed soy formula and 15 infants fed whey formula completed the study. Infants were put on cow-milk formula close to the time of hospital discharge. Body weight measurements were taken daily, and measurements of length and head circumference were taken weekly. Data were analyzed using Student $t$-test.

No significant differences were detected in calorie or protein intake or in formula tolerance between groups. At 8 weeks of age, the mean body weight of infants receiving whey formula was significantly higher than that of infants receiving soy formula. There was a significantly higher mean weight gain per day in the whey formula group during the last 2 weeks of the study, in addition to greater caloric efficiency at 7 weeks in this group. Similarly, mean weight gain over the duration of the study was significantly greater in the whey formula group. Mean serum protein and albumin concentrations were within the normal range for both groups, although values were significantly higher in the whey group at the end of the study. Mean serum calcium and phosphorus were significantly higher at 8 weeks in the whey formula group, although the values for both groups were within normal ranges. Concentrations of alkaline phosphatase, parathyroid hormone, and 25-hydroxyvitamin D were within normal ranges and no differences were detected between groups. No cases of rickets were identified in either group by wrist radiography.

The study authors concluded that premature whey formula was preferable to supplemented soy formula for very low birth-weight infants.

Strengths/Weaknesses: The prospective design, use of a homogeneous, special population sample of very low birth-weight infants, and the repeated measurements for growth including head circumference are strengths. Weaknesses include the small sample size and the initiation of subjects into the study at 3 weeks of age or older without adjustment for baseline differences.

Utility (Adequacy) for CERHR Evaluation Process: This study is not useful in the evaluation process.

Churella et al. (1994), support not indicated, conducted a controlled, randomized, blinded, parallel clinical trial of two soy formulas in 64 healthy term infants. Infants were randomly assigned to Isomil ${ }^{\circledR}($ protein $=2.91 \mathrm{~g} / 100 \mathrm{kcal})$ or a lower-protein $(2.45 \mathrm{~g} / 100 \mathrm{kcal})$ soy 
formula. Parents agreed to exclusively feed infants the assigned formula from approximately 2-112 days of age. Infants were evaluated at 8, 28, 56, and 112 days of age. Weight, length, and head circumference were measured at each evaluation. Blood samples were taken at 56 and 112 days and analyzed for plasma urea nitrogen, total protein, and albumin. Data were analyzed using ANOVA. No differences were detected between the groups in weight, length, or head circumference or in changes in these parameters. No differences in mean energy intakes calculated from 3-day formula intake records were detected. Mean protein intake was lower in the group fed the lower protein formula. Despite the significant differences in protein intake, no significant differences were detected between the two groups in total plasma protein concentrations, plasma albumin concentrations, or median plasma transthyretin concentrations. Plasma urea nitrogen concentration was lower in the low-protein formula group. The authors noted that the growth of the infants in their study was similar to that of infants in other studies who were fed human milk or cow-milk formula. They concluded that because transthyretin, a sensitive indicator of protein status, was present at similar concentrations in both groups of infants, the content and quality of the protein in both formulas was adequate for growing infants.

Strengths/Weaknesses: Strengths include the controlled randomized design, the exclusive feeding for duration of trial, the multiple measures of growth including head circumference, use of blood measures, follow-up to about 3 months of age, and, relative to other studies, the moderate sample size ( 32 in each group). The power to show a group difference was limited by sample size, however, and the comparison was only between soy formulas without a formal comparison to other kinds of formulas or to breast feeding.

Utility (Adequacy) for CERHR Evaluation Process: This study is not useful in the evaluation process.

Chan et al. (1987), supported by Ross Laboratories, randomized 10 male and 10 female term newborns per group to receive one of two soy formulas that differed only in carbohydrate source ( $100 \%$ glucose polymers compared to $50 \%$ glucose polymers $+150 \%$ sucrose). A comparison group of five male and five female newborns who were exclusively breast-fed was used for comparisons up to 4 months of age, and a historical control group was used for comparisons at 6 and 12 months. Breast-fed infants were given supplemental vitamins and fluoride. Weight, length, and head circumference were determined at 2 weeks, 2 months, and 4 months of age, and serum was obtained for total calcium, phosphorus, magnesium, 25hydroxycholecalciferol (a D-vitamin precursor), and alkaline phosphatase. Plasma and erythrocyte copper and zinc were measured, and bone mineral content (bone mineral density/ bone width) of the distal radius was measured [technique not specified, but probably singlephoton absorptiometry, which was in widespread use during the time period]. No differences were detected between groups in any of the measures of growth or serum chemistries. Plasma but not erythrocyte zinc was higher in breast-fed than formula-fed infants at 4 months (mean \pm SD $74 \pm 11 \mu \mathrm{g} / \mathrm{dL}$ in the breast-fed group compared to $63 \pm 15$ and $59 \pm 6 \mu \mathrm{g} /$ $\mathrm{dL}$ in the formula-fed groups). Bone mineral content was higher in the breast-fed group than in the formula-fed groups at 2 and 4 months. [Mean bone mineral content was estimated from a graph: 2 months $0.1 \mathrm{~g} / \mathrm{cm}^{2}$ in the breast-fed group compared to 0.08 and $0.06 \mathrm{~g} /$ $\mathrm{cm}^{2}$ in the formula-fed groups, 4 months $0.11 \mathrm{~g} / \mathrm{cm}^{2}$ in the breast-fed group compared to 0.08 and $0.06 \mathrm{~g} / \mathrm{cm}^{2}$ in the formula-fed groups. SEM seems to be about 0.02 for all data estimates.] At 6 and 12 months, bone mineral content was the same in the formula-fed infants as in the historical control group of breastfed infants. The authors posited that phytic acid (inositol hexaphosphoric acid) in soy formulas may interfere with calcium and zinc absorption, but that adaptation occurs and mineral absorption returns to normal over time. [No comment was made concerning the effects of supplemental fluoride on measured bone mineral density.] 
Strengths/Weaknesses: Strengths include random assignment by infant sex, use of two soy formulas and a breast-fed comparison group, multiple measures of growth including head circumference, and use of blood measures and bone mineral content. Weaknesses include the small sample size (10 in each group) and the lack of adjustment for confounders. The separate historical control group used for breast-fed comparisons at 6 and 12 months was not optimal, and there could have been selection bias for breast-fed infants.

Utility (Adequacy) for CERHR Evaluation Process: This study is of marginal utility in the evaluation process.

Venkataraman et al. (1992), supported by Ross Laboratories, evaluated growth and bone mineral content in 56 healthy term infants exclusively fed human milk or one of two study formulas until 4 months of age, when baby foods were added to their diets. Infants receiving human milk were also given vitamin D 400 IU daily. Seventeen infants received human milk, whereas the remaining infants were randomly assigned to cowmilk formula (Similac ${ }^{\circ}, n=19$ ) or soy formula (Isomil®, $\mathrm{n}=20$ ) using a block for equal sex distribution. The soy formula included modifications from previous soy formulas in calcium source and suspension characteristics. The feeding groups did not differ at the beginning of the study in weight, length, or head circumference. Infants were evaluated at 8, 16, and 24-26 weeks of age for length, weight, head circumference, bone mineral content (single photon absorptiometry of the distal radius), and serum concentrations of calcium, magnesium, phosphorus, alkaline phosphatase, and parathyroid hormone. Data were analyzed using $\chi^{2}$ for discrete variables and ANOVA or non-parametric methods for continuous variables. Tukey Student range test was used to evaluate differences between pairs.

No significant differences were detected between the three groups in growth measurements or in serum concentrations of calcium, magnesium, alkaline phosphatase, or parathyroid hormone at any time point. Bone mineral content and bone width were significantly greater in the soy group than in the human-milk group at 16 weeks, and the difference in bone mineral content remained significant at 24-26 weeks. Although serum phosphorus levels were lower in the human-milk group and in the cow-milk formula group at 8 weeks, differences did not remain significant at the 16- or 24-26-week evaluation times.

The authors suggested that decreased bone mineral content seen in previous studies in infants receiving soy formula could be attributed to decreased delivery of calcium salts to the infants due to sedimentation. They also suggested that the increased bone width and bone mineral content in soy formula-fed infants compared to breast-fed infants could be a result of lower nutrient density of human milk. The researchers concluded that the decreased bone mineralization associated with soy-formula feeding in infants could be prevented through improved suspension characteristics of the minerals used and also noted the importance of these characteristics in the interpretation of studies involving bone mineral status.

Strengths/Weaknesses: Strengths include the use of three feeding groups including a breastfed group, the random assignment of formula-fed infants, with procedures for assuring approximate equal distribution by infant sex, the multiple measurements of growth including head circumference measured and bone mineral content, and the restriction of solid foods during most of the study period. There were, however, apparently no adjustments for confounders. Selection bias may have occurred for breast-fed infants, and the sample was small with 17-20 in each group. There was a difference in fluid consumption between the groups.

Utility (Adequacy) for CERHR Evaluation Process: This study is of limited utility in the evaluation process. 
Mimouni et al. (1993), supported by the NIH, Children's Hospital Research Foundation, and Ross Laboratories, studied 72 healthy term infants receiving human milk $(\mathrm{n}=10)$, cow-milk formula (Similac $₫, n=20$ ), or one of two soy formulas (Prosobee ${ }^{\circledR}, n=21$ or Isomil ${ }^{\circledR} n=$ 21). Breast-fed infants were recruited from infants whose mothers had decided to breast feed and were given vitamin D 400 IU. Supplementation of feeds with cow-milk formula was permitted. Infants whose mothers had decided not to breast feed were randomly assigned to a formula group. Solid food was withheld until 3 months for breast-fed infants and until 4 months for formula-fed infants. The infants were examined at entry into the study (2-7 days of age) and then at $8,16,26$, and 52 weeks for measurement of weight, length, head circumference, bone mineral content (single-photon absorptiometry, distal radius and ulna), and serum calcium, phosphorus, magnesium, alkaline phosphatase, and 1,25-dihydroxyvitamin D. Data were analyzed using $\chi^{2}$ for discrete variables and ANOVA or non-parametric methods for continuous variables. Tukey Student range test was used to identify pair-wise differences.

No differences in weight and head circumference by feeding group were detected, but length gain was significantly lower in the breast-fed group than in the three formula-fed groups from 1-26 weeks. Bone mineral content and bone width were similar between groups throughout the study. No significant feeding group effects on levels of serum total or ionized calcium, magnesium, or alkaline phosphatase were detected. Serum phosphorus was significantly lower in the breast-fed group than in the combined formula groups at 8 weeks and lower than in the cow-milk formula group at 26 weeks. Serum levels of 1,25-dihydroxyvitamin D were higher in the Prosobee ${ }^{\circledR}$ group than in the other groups at 8,16 , and 26 weeks.

The authors suggested that elevated concentrations of 1,25-dihydroxyvitamin D in Prosobee $®$ fed infants could indicate inadequate mineral intake or high mineral need. They indicated, however, that the increase in 1,25- dihydroxyvitamin $\mathrm{D}$ was apparently sufficient to increase calcium absorption and resulted in adequate bone mineral content. The authors concluded that the concentration and suspension characteristics of minerals in available soy-based formulas permitted adequate growth and bone mineralization.

Strengths/Weaknesses: Strengths include the use of four feeding groups, including two types of soy formula, and random assignment to formula-fed groups. The 1-year follow-up and multiple measurements of growth including head circumference and bone mineral content are additional strengths. There could have been selection bias for breast-fed infants, however, and supplementation with cow-milk formula was allowed for breast-fed infants. Solid foods were restricted for differing lengths of time in breast-fed and formula-fed infants and were introduced early. The sample size was relatively small.

Utility (Adequacy) for CERHR Evaluation Process: This study is of limited utility in the evaluation process.

Stettler et al. (2005), supported by the NIH and the International Formula Council, evaluated the relationship between rate of weight gain in early life and overweight in adults. Subjects included adults (20-32 years old, median age $=26$ years $)$ who had participated in infant feeding studies between 1965 and 1978 in Iowa. The feeding studies had been restricted to infants who were not breast-fed at all. In these studies, assignment was made to whichever cow-milk or soy formula was being studied in the research unit at the time of the child's birth. Infants were enrolled at 8 days of age and were followed to 112 days of age. The adults who were subsequently recruited for follow-up had eaten their assigned formula for the duration of the study period. Additional foods were permitted after 28 days of age. From the 952 children in the original infant studies, 653 adults were located, met inclusion criteria, and agreed to participate. The subjects were contacted by telephone. Information was obtained from the subjects on current height and weight and on whether the parents of the subjects were 
overweight. The adult subjects were considered overweight if their body-mass index was $\geq 25$ $\mathrm{kg} / \mathrm{m}^{2}$ and obese if their body-mass index was $\geq 30 \mathrm{~kg} / \mathrm{m}^{2}$. [Because there were not enough obese subjects for planned analyses, the analyses involved overweight subjects, who represented 32\% of the cohort.] Infant weight had been obtained at 7 intervals through 112 days of age and was expressed corrected for age as a $z$-score. The period between birth and 8 days of age was identified as the most sensitive for predicting adult overweight, and subsequent analyses focused on this time period. The relationship between adult overweight and weight gain during this period was evaluated by multiple logistic regression with respect to potential confounders.

The unadjusted analysis showed absolute weight gain during the first week of life and from birth to 112 days to be significantly associated with adult overweight. Use of soy formula was not significantly associated with adult overweight in this analysis. With adjustment for birth weight, overweight parents, age in adulthood at the time of the survey, subject income, and first-week infant weight gain, there was a significant association between use of soy formula and adult overweight $(\mathrm{OR}=1.47,95 \% \mathrm{CI}=1.01-2.13, P=0.046)$. When infant weight gain from birth to 112 days of age was included in the model, statistical significance was lost (OR $=1.37,95 \% \mathrm{CI}=0.95-1.98, P=0.1)$. The authors called the association between soy formula and overweight an incidental finding and suggested that it be treated with caution due to the inconsistency in results between the analytic models and due to the borderline level of statistical significance. They concluded that "soy-based formulas should be further investigated as a possible risk factor for overweight."

Strengths/Weaknesses: Strengths are that infants were exclusively formula-fed for at least 28 days and were quasi-assigned to soy or cow milk by site rather than by mother or pediatrician preference. The multivariate analyses addressed a variety of other factors in infancy that could have been important predictors of adult overweight. If soy formula really is associated with adult overweight relative to cow-milk formula, adjusting for the higher infant weight gain up to 112 days of age (the intervening variable) in the multivariate model would have removed some of the effect of soy formula. Weaknesses are the relatively small proportion of subjects available for follow-up, the lack of information on other potential confounders that could have been important in the 26 years to follow-up but were not measured, the lack of specific information on solid food that was given between 28 and 112 days, and the lack of information on the reasons for mothers selecting exclusive formula feeding. Infants usually lose weight in the first week and regain their birth weight by 2 weeks of age, so weight gain during the first week is not a usual observation in clinical practice.

Utility (Adequacy) for CERHR Evaluation Process: This study is useful for the evaluation process.

3.1.2.2 Gastrointestinal effects: Reports on gastrointestinal effects of soy formula are presented. Case series are followed by controlled studies. Some reports include what may be gastrointestinal manifestations of allergic disease, and these studies might just as reasonably have been discussed in Section 3.1.2.3.

Ament and Rubin (1972), supported by the NIH, the Public Health Service, the Children's Orthopedic Hospital and Medical Center, and the University of Washington, presented a case of a 6-week-old infant with an immediate response to soy feeding consisting of fever, leukocytosis, cyanosis, vomiting, blood-tinged mucoid diarrhea, dehydration, and metabolic acidosis, all of which disappeared after 3 days of i.v. feeding. Subsequent challenges with soy protein were associated with violent systemic symptoms and jejunal abnormalities on endoscopy consisting of loss of villi and surface ulcerations. There was no reaction to gluten, soy lecithin, lactose, or sucrose. A moderate reaction to cow milk disappeared by 10 months 
of age, whereas the soy reaction continued to occur. The authors suggested that this response was unlikely to be allergic. They noted a similarity of the intestinal lesion to that of celiac disease and wondered if the underlying mechanism of injury might similarly be based on a genetic abnormality.

Strengths/Weaknesses: This study represents a single case report of what may be a unique reaction.

Utility (Adequacy)for CERHR Evaluation Process: This report is not useful in the evaluation process.

Iyngkaran et al. (1988), funding not indicated, investigated the effects of soy protein on the small bowel mucosa of 18 infants who suffered from acute gastro-enteritis. Infants were hospitalized for correction of fluid and electrolyte status, after which they received a protein hydrolysate formula (such as Pregestimil ${ }^{\circledR}$, Nutramigen ${ }^{\circledR}$, Alfare ${ }^{\circledR}$, or prehydrolysed human milk) for 6-8 weeks. Infants were readmitted again for soy-protein challenge (Prosobee ${ }^{\circledR}$ ) and evaluated by biopsy for histologic appearance and enzyme levels in the jejunal mucosa before and $20-24 \mathrm{hr}$ after the challenge. Ten (55.5\%) of 18 infants developed soy-protein enteropathy. The infants were divided into three groups based on their histologic and clinical results. Group $1(\mathrm{n}=3)$ showed histologic and clinical reactions between the third and ninth hour of soyprotein challenge. Histologic reactions included depletion of the mucosal enzymes lactase, sucrase, maltase, and alkaline phosphatase and depressed blood xylose levels; clinical reactions were diarrhea, weight loss, vomiting, lethargy, fever, rhinitis, and skin rash. In Group 2 ( $\mathrm{n}=$ 7), infants experienced only histologic reactions to the initial challenge. As a group, they had less depression of mucosal enzyme activity and blood xylose absorption compared to Group 1 , but individual reactions varied considerably. Group 3 (eight infants) did not have any histologic, enzymatic, or clinical reactions to the soy-protein challenge. Significant differences in mucosal enzymes and xylose absorption were not found on comparison of Groups 1 and 2 to Group 3.

Strengths/Weaknesses: The clinical evaluation after soy challenge and the inclusion of biopsies are strengths, but this sample is highly select group of children with acute gastrointestinal episodes apparently due to a variety of causes. It was not possible to determine exactly which of the 18 infants had been exposed previously to soy before the acute episode and soy challenge. There was no comparison group.

Utility (Adequacy) for CERHR Evaluation Process: This report is not useful in the evaluation process.

Halpin et al. (1977), funding not indicated, reported four infants, aged 2-4 months, with suspected intolerance to soy protein. Infants were admitted to the hospital, and proctosigmoidoscopy with rectal biopsy was carried out within $24 \mathrm{hr}$. Gross and microscopic evidence of acute colitis was present in all four infants. After 1 month, all infants had recovered and sucrose and lactose tolerance tests were normal. Proctosigmoidoscopy with biopsy was repeated to confirm mucosal normalcy. Soy-protein challenge was given by feeding increasing amounts of soy formula (Isomil®) over $8 \mathrm{hr}$. If the feeds were tolerated, the soy formula was continued every $4 \mathrm{hr}$ for up to 5 days. Stools were weighed and tested for occult blood and for the presence of reducing sugars. If the stool results were abnormal, a proctosigmoidoscopy with rectal biopsy was carried out. Soy-protein challenge produced symptoms in the infants within $3 \mathrm{hr}$ to 5 days. Proctosigmoidoscopy showed friable mucosa, loss of vascular pattern, acute colitis (polymorphonuclear leukocytes present in the lamina propria or in the walls of the rectal glands), and an increase in mitotic cells in the crypts. Other findings included frank mucosal hemorrhage (two infants), crypt abscess (one infant), and depleted rectal-gland mucus 
(two infants). Abnormal stool tests (low $\mathrm{pH}$ and increased reducing sugars) occurred in two infants, but these infants had no symptoms and their biopsies were normal. The authors suggested that the normal biopsies could be a result of rapid recovery, mild infection, or patchy mucosal damage. When biopsies from symptomatic children were evaluated blindly against biopsies from children with other colitides, the authors were unable to distinguish infectious colitis or mild ulcerative colitis from soy protein-induced colitis. They were able to distinguish severe ulcerative colitis and granulomatous colitis, nodular lymphoid hyperplasia, and normal bowel from the specimens taken in this study. The authors concluded that soy protein-induced intestinal mucosal damage is under-reported, and that soy protein may have the same potential for mucosal injury as does cow-milk protein.

Strengths/Weaknesses: In this case series, all four infants were known to have been fed soy formula and were suspected to be intolerant. The withdrawal and soy protein challenge under controlled conditions is a strength, but there was no comparison group. This population may represent a uniquely susceptible subgroup.

Utility (Adequacy) for CERHR Evaluation Process: This report is not useful in the evaluation process.

Powell (1978), support not indicated, reported nine infants, aged 4-27 days at onset of symptoms, with protracted diarrhea before and after milk and soy-formula challenges. A control group consisted of 11 infants (age $=2-9$ months) who were hospitalized for chronic diarrhea not caused by cow-milk or soy formula. [Not all of these control infants were tested in the same manner as the formula-intolerant infants, and results for the control infants are not clearly presented.] Infants in both groups were managed with i.v. therapy if needed and with a casein hydrolysate formula (Nutramigen $\left.{ }^{\circledR}\right)$. Once normal stools were observed and appropriate weight was gained, the children were challenged with cow-milk formula and, at least 1 week later, with soy formula. Stools were tested $12 \mathrm{hr}$ before the challenge and $48 \mathrm{hr}$ after the challenge. Complete blood counts, serum $\beta 1 \mathrm{C}$ globulin, and serum protein electrophoresis were determined before and after the challenge. A positive response to the challenge was defined as liquid stools or stools that contained blood or leukocytes within 24 hr of the challenge. In the group with formula intolerance, eight of nine infants had positive responses to the cow-milk challenge and six of nine had positive responses to the soy challenge. Serum protein electrophoresis remained unchanged in all but one infant. There was a statistically significant increase in serum $\beta 1 \mathrm{C}$ globulin levels after the positive responses 90 min after the challenge but not thereafter. Leukocytosis occurred 6-10 $\mathrm{hr}$ after the positive challenges. No changes in eosinophils ( $<6 \%$ of white blood cell count), hemoglobin, or hematocrit values were observed.

The authors concluded that the severity of responses may have been unique to this age group. They also concluded that soy formula can be just as damaging as cow-milk formula if fed during this stage in the infant's life or after a reaction to cow milk.

Strengths/Weaknesses: This study included infants with known cow-milk or soy protein gastrointestinal sensitivity. A comparison group with similar symptoms that were known not to be due to formula was used, and challenge with both cow-milk and soy formula occurred in each child. Weaknesses include the apparent nonrandom order of challenge (cow milk and then soy) and it was not clear if the control group received the same challenges and what the results were.

Utility (Adequacy) for CERHR Evaluation Process: This study is not useful in the evaluation process. 
Lothe et al. (1982), funding not indicated (but formula provided by Mead Johnson), carried out a double-blind crossover study to determine the effect of cow milk on infantile colic. Sixtyfive cow-milk formula-fed infants with symptoms of infantile colic were identified at the Children's Hospital in Malmö, Sweden and enrolled. Sixty infants between the ages of 2 weeks and 3 months completed the study. Identical-appearing cans of formula were provided to the parents for use over a 2-week period. The cans contained 1 week of cow-milk formula (Enfamil $\left.{ }^{\circledR}\right)$ or 1 week of soy formula (ProSobee $\left.{ }^{\circledR}\right)$. [Randomization of order was not discussed.] Five infants also received some human milk, and their mothers were instructed not to drink cow milk during the study period. Parents completed a daily questionnaire noting symptoms of colic (including paroxysmal abdominal pain, sustained severe crying, distended abdomen due to gas, and frequent wish to suck). Parents were also asked to note "whether the infant seemed to thrive" on the test formula. Infants who seemed to have improved with soy the first week and then worsened the second week on cow milk were put back on soy formula at the end of the study period. Infants who received soy formula the second week and seemed to improve were given a cow-milk formula challenge 1,3 , and 9 months later to exclude spontaneous recovery. Infants whose symptoms did not improve with either the soy or cowmilk formulas were then given a hydrolyzed casein formula (Nutramigen ${ }^{\circledR}$ ) containing neither cow milk nor soy protein. Infants who improved on the casein hydrolysate formula were also rechallenged with cow-milk formula [results not discussed here].

Seventeen infants (29\%) had what seemed to be a spontaneous recovery and tolerated the cowmilk formula. In 11 infants (18\%) who did not tolerate cow-milk formula, colic disappeared within $48 \mathrm{hr}$ of being put on soy formula. On rechallenge with cow milk 1 month later, 8 of 11 infants had recurrent symptoms. Of the remaining 32 infants (53\%) whose symptoms did not diminish on either cow-milk or soy formula, all were symptom-free within $48 \mathrm{hr}$ of being placed on casein hydrolysate formula, and 14 of 32 infants had recurrent symptoms when rechallenged with cow milk.

The authors concluded that cow milk is a significant factor in the development of infantile colic in formula-fed infants. They hypothesized an allergic cause and suggested dietary treatment with alternative formulas, such as those based on soy or casein hydrolysate.

Strengths/Weaknesses: All infants were said to have severe colic and to have been fed with cow milk-based formula. Infants were treated with both cow-milk and soy formula successively, with the mother blinded to formula content. To rule out spontaneous recovery, infants who recovered were challenged again later with cow milk formula. It was unclear, however, how formula was assigned and if it made a difference which formula was given first. Later challenges to rule out spontaneous recovery were only carried out with cow milk even if the infant had not improved on soy formula. In addition, there was no inclusion of a group of infants without colic.

Utility (Adequacy) for CERHR Evaluation Process: This report is not useful in the evaluation process.

Poley and Klein, 1983, supported by NIH, reported observations from scanning electron microscopy and light microscopy of small intestine biopsies from two infants with soy proteininduced villous atrophy. The first biopsy was carried out on admission, and a follow-up biopsy was carried out 6 weeks later. Mucosa obtained from a 7-month-old infant who had been on a sucrose-restricted diet with no diarrhea for 4 weeks preceding biopsy was used as a control. The soy-sensitive patients showed flattening of the villi, indistinct cell borders, lack of glycocalyx, and exposed microvilli. The degree of villous atrophy was similar to that seen in gluten-sensitive enteropathy. The authors estimated that 50-75\% reconstruction of villous height had occurred at the 6-week follow-up biopsies. Tissue disaccharidase activity 
[measured as the production of glucose after incubation of disaccharide with a tissue homogenate] was depressed during soy feeding but showed recovery after 6 weeks. The authors described the recovery after cessation of soy exposure as "remarkable."

Strengths/Weaknesses: The detailed evaluation of the clinical and histologic picture of the disease and course of recovery is a strength, as is the inclusion of a control patient. It is a weakness that only two infants were included.

Utility (Adequacy)for CERHR Evaluation Process: This report is useful and shows that some infants are sensitive to soy protein.

Burks et al. (1994), supported by NIH and Ross Laboratories, studied 43 infants under the age of 18 months to evaluate whether formula protein source was associated with enterocolitis. All subjects were suspected to have milk- or soy-protein enterocolitis due to vomiting or other gastrointestinal symptoms. Patients with suspected IgE-mediated adverse food reactions were excluded. Formula challenges were carried out in the hospital when the children were symptomatic. The challenge groups were:

- Group $1(\mathrm{n}=23)$ : cow-milk formula, powdered soy formula, liquid soy formula,

- Group $2(\mathrm{n}=12)$ : cow-milk formula, liquid soy formula, powdered soy formula, and

- Group $3(\mathrm{n}=8)$ : cow-milk formula, then soy formula challenges in randomized order.

[The grouping was not part of the original study design; study investigators modified the order of formula administration after results from the first group failed to support their hypothesis that the last formula would be most likely to produce a reaction. Thus, testing of the three groups was successive, and only the last group had a randomization component.]

The subjects fasted for 4-6 hr before challenge, and there was $48 \mathrm{hr}$ between challenges. Patients were observed and stools were collected during the $24 \mathrm{hr}$ after challenge. Reactions were considered positive if two or more of the following criteria were met: (1) vomiting or diarrhea developed; (2) absolute neutrophil count increased 6-8 hr after the challenge by at least $3500 / \mathrm{mm}^{3}$ compared to blood drawn immediately before challenge; (3) blood was identified in stool; (4) leukocytes were present in Wright-stained fecal mucus; and (5) CharcotLeyden crystals or eosinophilic debris were noted in stool after Hansel staining. Laboratory personnel were blinded to formula type. Statistical analysis methods were not given in detail. [Subjects seem to have been combined without regard to group.]

Twenty-one infants (48.8\%) had a negative challenge to milk and soy formulas, 12 (27.9\%) reacted only to soy, four (9.3\%) reacted only to cow milk, and six (14.0\%) reacted to both cow milk and soy. [Statistical comparisons were not reported; chi squared test by CERHR gives $\boldsymbol{P}=0.1$ for any soy reaction compared to any cow-milk reaction.] Patients who reacted to only one type of soy formula tended to react to whichever formula was administered first. Because the order of administration rather than the type of protein isolate was associated with enterocolitis, the authors concluded that processing soy into a powdered form did not render it less likely to cause a reaction. The authors interpreted the ordering effect as evidence that milk- and soy-protein enterocolitis may be caused by an unknown local immune mechanism with depletion of gastrointestinal immune mediators by the first challenge, regardless of protein source.

Strengths/Weaknesses: Strengths include the evaluation of all infants with enterocolitis thought to be due to formula intolerance and sequential administration of three types of protein. The non-random order of the challenge sequence and the lack of accounting for formula type at baseline are weaknesses. 
Utility (Adequacy) for CERHR Evaluation Process: This report is somewhat useful in the evaluation process.

Ostrom et al. (2006), supported by Abbott laboratories, evaluated the effect of a high-fiber soy formula on regurgitation in healthy infants whose parents reported that more than $25 \%$ of feedings were associated with regurgitation. Formula-fed infants were enrolled at 13-32 days of age and randomized to receive a cow-milk formula (Similac ${ }^{\circledR}$ With Iron, $n=90$ ) or a soy formula with $6 \mathrm{~g}$ added soy fiber (Isomil ${ }^{\circ} \mathrm{DF}, \mathrm{n}=89$. Parents recorded the incidence and estimated volume of regurgitation during the first 7 days and the last 3 days of a 28-day feeding period. Study completion rates were $74-76 \%$ with formula intolerance as the most common reason for discontinuation. The number of regurgitation episodes decreased in both groups; however, the soy formula-fed group had fewer daily regurgitations at 7 days and at 28 days than the cow-milk formula group. The mean \pm SEM number of daily regurgitations went from $3.6 \pm 0.2$ at baseline to $2.4 \pm 0.3$ on study day 28 in the cow milk-formula group and from 3.9 \pm 0.2 at baseline to $2.0 \pm 0.2$ in the soy-formula group.

Strengths/Weaknesses: The study design and sample size were adequate; however, the inclusion and exclusion criteria based on history were vague. Infants were excluded for a history of pyloric stenosis, but pyloric stenosis may not become evident until 4-6 weeks of age. The volume of regurgitation was not well quantified, but may have been inconsequential if all infants thrived. Length and head circumference measurements would have been helpful in this regard. It was unclear if the $10 \%$ of women who refused baseline measurement and the $25 \%$ of remaining women who did not complete this relatively short trial influenced the results. It is curious that in study Figure 2, there seems to be a significant difference in regurgitation frequency between treatment groups on Day 1 of the intervention.

Utility (Adequacy) for CERHR Evaluation Process: This study is somewhat useful in the evaluation process.

3.1.2.3 Allergy and immunology: Mortimer (1961), funding not indicated, reported a case study of soybean anaphylaxis in a male child with asthma and eczema. The anaphylactic reaction occurred at 20 months of age. The child had previously been exposed to soy formula, and the family lived near a soy-processing facility. At age 3 years, the child was slowly reintroduced to soy milk to test the hypothesis that allergenicity would decrease with avoidance of antigen. No reaction was seen. The author concluded that soybean allergenicity should be re-examined to determine the potential for soy to approach cow milk as an allergen in light of increasing exposure in the general population.

Strengths/Weaknesses: This case report is described in detail, but it is still just a case report.

Utility (Adequacy) for CERHR Evaluation Process: This study is not useful in the evaluation process.

Whitington and Gibson (1977), support not indicated, presented case reports on four infants with soy-protein intolerance. The four infants developed diarrhea during the first month of life while on cow-milk formula. Switching to soy-based formula resulted in clinical deterioration. Responses to soy-challenge tests included diarrhea, vomiting, hypotension, lethargy, and fever. Switching to a diet free of soy or cow-milk protein was followed by recovery and weight gain.

Strengths/Weaknesses: This case series involves severe clinical courses in infants sensitive to soy formula. It is a weakness that all infants first showed sensitivity to cow milk formula before responding poorly to soy formula. 
Utility (Adequacy) for CERHR Evaluation Process: This study is not useful in the evaluation process.

Perkkio et al. (1981), supported by the Finnish Foundation for Pediatric Research and the Paolo Foundation, conducted a morphometric and immunohisto-chemical study of jejunal biopsy specimens from five infants with soy allergy, all of whom also had cow-milk allergy. The biopsies were taken when soy was the only food source for the infants, and they were compared to biopsies taken before soy feeding and after soy had been eliminated from the diet. Biopsies were either processed for routine histology or examined by direct immunofluorescence or by the direct immunoperoxidase technique. In four infants, serial blood samples were taken for measurement of immunoglobulins, complement fractions, and soy antibodies. During soy feeding, biopsies of the five study patients showed partial or total villous atrophy associated with crypt hyperplasia. Inflammation of the lamina propria and the epithelium was seen and was similar both to that seen in intestinal cow-milk allergy with malabsorption and to that seen in celiac disease. There were also increased numbers of plasma cells and IgA- and IgM-containing cells in the lamina propria, as well as increased numbers of intraepithelial lymphocytes. Soy antibodies appeared and IgA also increased in the serum at the time of the reaction to soy. When soy feeding was stopped, the morphology of the jejunum improved and cell numbers were reduced to normal. The authors concluded that intestinal soy allergy was similar to intestinal cow-milk allergy and was readily reversible.

Strengths/Weaknesses: This study included careful clinical and histologic descriptions of infants with gastrointestinal sensitivity to soy formula, including comparison of biopsies before and after soy feeding and demonstration of reversal of changes. It is a weakness that all infants had known previous cow-milk formula sensitivity as well.

Utility (Adequacy) for CERHR Evaluation Process: This study is not useful in the evaluation process.

Halpern et al. (1973), supported by Borden, Inc., followed 1753 Caucasian infants (893 male) who were fed either human milk, soy formula, or cow-milk formula from birth to 6 months of age to observe the influence of diet, family history, and sex on the development of childhood allergy. Assignment to the three dietary groups was not randomized, but was as "unprejudiced as possible if the mother did not insist on a particular regimen." Breast feeding occurred in 352 infants, soy formula was given to 317 infants, and cow-milk formula was given to 1081 infants. The soy formula was Mull-Soy®, and the cow-milk formula was a proprietary liquid formula (Bremil ${ }^{\circledR}$, Similac $\AA$, Enfamil $®$, or SMA $\left.®\right)$. The mothers of the breast-fed infants were advised to limit their own cow-milk intake to 1 pint per day. The infants were followed for varying periods of time up to age 7 years. Diagnosis of food allergy was made solely on clinical grounds, with at least three episodes of characteristic symptoms being required to verify each diagnosis. Of 1753 children, 401 were diagnosed as either definitely or possibly allergic. Children with a family history of allergy were significantly more likely to develop allergy, but diet had no significant effect on the development of childhood allergy. The authors noted that only $0.5 \%$ of infants fed soy milk developed allergy in the first 6 months of life, whereas $1.8 \%$ of infants fed cow milk became allergic to it $\left(P<0.05, \chi^{2}\right)$. The authors concluded that this finding agreed with the conclusions of other investigators that "soy milk is a less potent sensitizer."

Strengths/Weaknesses: Strengths include a large sample size from nine different pediatric offices with three feeding groups and prospective ascertainment and follow-up for as long as 7 years. The authors evaluated a broad spectrum of allergic endpoints and included family history. There was, however, a lack of randomization to feeding group; women who had a preference could choose method of feeding and more women with immediate family history of allergy chose soy formula. Pediatrician preference also played a role. Many of the groups 
were mixed-feeding groups and mothers could have changed methods of feeding during the study period. Infants were followed for varying lengths of time, and there was substantial attrition at each follow-up point. No time variable was included in the analyses, and the denominator for the incidence of allergy was the entire sample regardless of the length of follow-up. Not all children were evaluated for allergies by a specialist, only those referred by the pediatrician. Multivariate analysis should have been used to control for multiple potential confounders simultaneously.

Utility (Adequacy) for CERHR Evaluation Process: This study is not useful in the evaluation process.

Kjellman and Johansson (1979), supported by Förenade Liv, Semper Fund for Nutritional Research, Linköping University, and the Swedish Medical Research Council, carried out a randomized clinical trial of soy formula $(n=23)$ or cow-milk formula $(n=25)$ in children with biparental atopic disease. Mothers were encouraged to breast feed, and infants were placed on a randomly assigned formula from weaning until age 9 months. At age 9 months, soy formulafed infants were slowly introduced to cow milk. Children were followed to age 4 years. They were evaluated periodically, and atopic disease was rated as obvious, probable, possible, or none based on the number of episodes of atopic dermatitis, bronchial asthma, allergic rhinitis, allergic urticaria, or gastrointestinal allergy. Cord blood and venous blood drawn at 3, 9, 12, 18 , and 36 months of age were analyzed for $\operatorname{IgE}$ and IgG antibodies to cow-milk and soybean protein and for levels of IgE, IgG, IgA, IgM, hemoglobin, cholesterol, and albumin. Statistical methods were not discussed.

Obvious or probable atopic disease occurred in two-thirds of children during the 4-year follow up period. There was no statistically significant difference in development of obvious or probable atopic disease by sex or formula type. Four children in each formula group were found to have IgE antibodies to cow milk. IgG antibodies to $\beta$-lactoglobulin, a protein, were present in the cord blood of all but 3 infants. The cow-milk formula group had significantly higher levels of IgG antibody to $\beta$-lactoglobulin from age 3-18 months, but the difference was no longer statistically significant at 3 years (Table 24). [The Expert Panel cannot evaluate these data because the graph in the original article did not indicate variance, and the statistical methods were not given.]

IgE antibodies to soybean protein were not shown to differ significantly between groups. Serum $\operatorname{IgG}$ antibodies to soybean protein were significantly higher at 9 and 12 months of age in the soybean formula group: mean \pm SD $65.7 \pm 164.1$ (9 months) and $63.3 \pm 104.5 \%$ ( 12 months) of reference serum compared to the cow-milk formula group: $9.5 \pm 2.0$ (9 months) and $24.5 \pm 52.5 \%$ (12 months) of reference serum. No differences in IgE levels were detected between healthy and atopic children, and no significant differences were detected at birth or at 3, 18, or 36 months.

Overall, there were very few side effects of soy-formula intake, and all children in the soy group tolerated the formula, except for one child with a malabsorption syndrome who was put on a soy-free diet until age 3 years. The authors concluded that delaying introduction of cow milk by feeding infants soy formula until 9 months of age did not diminish the incidence of cow-milk intolerance.

Strengths/Weaknesses: The children in this study had a strong family history of allergy. Strengths include the random assignment to formula-feeding group and specified time that children were fed in that manner. There was long-term follow-up with repeated blood measures of immune status. The small sample size is a weakness. There was no breast-fed comparison group, and all infants were breast-fed for varying lengths of time before being randomized to 
formula group. Mothers could mix breast feeding and formula feeding, making effects of one food source impossible to determine. Statistical methods were not shown.

Utility (Adequacy) for CERHR Evaluation Process: This study is not useful in the evaluation process.

Gruskay (1982), support not indicated, conducted a prospective evaluation of 908 children, 328 of whom had a family history of major allergy, and a control group of 580 children with no such family history, with the objective of determining if diet during the early months of life was related to the development of atopy later in childhood. The study population consisted of consecutive, healthy full-term newborns under the care of the author or participating pediatricians. In the families with a history of allergy, breast feeding was chosen by the mothers of 48 subjects. The remaining infants were assigned non-randomly to soy formula (every fourth child, $\mathrm{n}=79)$ or cow-milk formula $(\mathrm{n}=201)$ [formula composition not given]. Children were evaluated every month from 1 to 6 months of age, then every 2 or 3 months until age 2 , and every year for up to 17 years thereafter. Children without a family history of allergy all received cow-milk formula if bottle-feeding was elected by the mother. Comparisons were made in the proportion of children at different ages with allergy. Statistical procedures were not discussed.

After 15 years, $50 \%$ of infants with a family history of allergy had developed allergic disease, whereas only $15 \%$ of control infants had developed allergy [level of significance not given]. In infants with a positive family history, allergy developed over 15 years with the same frequency in those fed cow-milk formula as in those fed soy formula (53\%). Allergy was present significantly less often in children with a family history of allergy if they had been breast-fed, but differences in allergy rates between bottle- and breast-fed infants did not become statistically significant until after 3 years of age. The author concluded that breast feeding may play a role in decreasing the incidence of allergic disease. He also concluded that soy formula has no apparent advantage over cow-milk formula in the prevention of allergy.

Strengths/Weaknesses: Strengths include the large sample size with both positive and negative family history groups. There was some attempt to randomly assign mothers in the positive family history group to soy or cow-milk formula groups. The long-term follow-up is a strength, although the statistical methods did not include time in follow-up. The authors seemed to use appropriate denominators at each follow-up time point. Weaknesses include the possible selection bias inasmuch as mothers could elect to breast feed. Mixed feeding was allowed, and there was no adjustment for other foods when introduced. In the end, it seems authors only compared soy formula-fed to breast-fed infants in the positive family history group. Soy formula was not formally compared to cow-milk formula, although rates of allergy incidence were similar overall in both soy and cow-milk formula groups. There was no adjustment for potential confounders.

Utility (Adequacy) for CERHR Evaluation Process: This study's utility is limited to a comparison of soy formula feeding and breast feeding in infants with a positive family history.

Moore et al. (1985), supported by the U.K. Medical Research Council, conducted a study of the breast-fed infants of atopic parents. The study objective was to determine the effectiveness of breast feeding in the prevention of atopy. Women in an experimental group $(n=250)$ were asked to breast feed their babies for the first 3 months, avoid giving them solids, and to use a soy formula if any extra food was necessary. Women in the control group $(n=275)$ used a cow-milk formula. The infants were seen daily until their discharge from the hospital and then at 3,6 , and 12 months. The mothers kept feeding diaries during the first 3 months. At 3 months, detailed feeding histories were obtained from the mothers, and the infants were clinically examined. The proportion of infants having eczema at 3 months of age was related to the time 
they were introduced to cow milk, with more than twice as many of those who were given cow milk in the first 4 weeks developing eczema as those introduced to cow milk during weeks 58 [level of significance not indicated]. Significant differences were found in the prevalence of eczema at 3 and 6 months between infants who had received cow milk in Weeks $1-4$ and those who had not. No significant differences were detected between groups at 12 months. Multivariate analysis identified breast feeding as a protective factor. There was no detectable advantage of using soy as opposed to cow-milk formula as an artificial food source.

Strengths/Weaknesses: Strengths include use of a homogeneous group with positive history of eczema or asthma. Women who agreed to participate could be randomly assigned to breast feeding for at least 3 months or to standard advice. The multivariate analysis and the initially large sample size are additional strengths. The study was, however, primarily focused on comparing breast feeding to cow-milk formula. It seems that few women actually fed their infants soy formula, and almost none used this method exclusively. Consequently, the sample was not adequate for a comparison of soy formula-fed infants to cow milk-formula or breastfed infants.

Utility (Adequacy) for CERHR Evaluation Process: This study is not useful in the evaluation process.

Miskelly et al. (1988), supported by Wyeth Laboratories and the Welsh Scheme for the Development of Health and Social Research, compared the effects of feeding cow-milk or soy formula on the development of allergic symptoms in 487 infants at risk of allergic disease because of family history. Women randomized to the intervention group were advised not to give their infants cow milk and to restrict their own cow-milk intakes to a half-pint per day. They were supplied with a soy formula to supplement or replace breast feeding. Mothers in the control group were not given instructions on any diet restrictions, and most infants in this group received cow milk. Babies were examined for allergic symptoms at 3, 6, and 12 months by a physician who was unaware of the babies' formula types. Skin tests for milk, cod, egg, wheat flour, and soy antigens were carried out at 6 and 12 months. Feeding diaries were kept by the mothers. The proportions of infants with symptoms were evaluated using a $\chi^{2}$ test with Yates correction.

Mothers in the intervention group were less likely to breast feed than those in the control group. The authors suggested that the decreased breast feeding in the intervention group was due to the free supply of soy formula given to women in this group. The only significant symptomatic difference detected in the first year of life between the two groups was in the incidence of oral thrush (51\% soy formula compared to $40 \%$ breast feeding, $P<0.05)$. When the effects of breast feeding were evaluated, infants who were never breast-fed had a significantly higher incidence of wheezing and of diarrhea than infants who were breast-fed. There was also a numerically higher incidence of nasal discharge (not statistically significant). Skin testing showed that the only food antigen associated with eczema was egg, with positive tests occurring in $15 \%$ of babies with a history of eczema compared to $2 \%$ of babies without eczema $(P<0.001)$. The authors concluded that breast feeding was highly advantageous and that their findings gave no support to the hypothesis that withholding cow milk reduces the risk of allergic disease.

Strengths/Weaknesses: Strengths include the large sample size and the homogeneous group of infants at risk of allergy due to family history. The intervention was a combination of breastand soy formula-fed infants, which is an important weakness. There does not seem to have been a separate analysis for soy formula-fed infants, so lack of differences between groups could have been due to failure to compare subgroups of the intervention group to the control group. 
Utility (Adequacy) for CERHR Evaluation Process: This study is of marginal utility in the evaluation process.

Chandra et al. (1989a), supported by the National Health Research Development Programme of Health and Welfare Canada, and the Mead Johnson Canada division of Bristol Myers, examined the effects of maternal diet during breast feeding and the effects of three different types of formula on the development of atopic eczema in infants with family histories of atopic disease. A mother was recruited if either she or the baby's father had a family history of atopy. Mothers planning to breast feed exclusively were randomly assigned to an unrestricted-diet group or an experimental group asked to exclude dairy products, eggs, fish, peanuts, and soybeans from their diet and to take a $1 \mathrm{~g}$ calcium supplement daily for 6 months or the duration of lactation. Compliance with the requested restrictions was assessed by questioning, analysis of food diaries, and testing for $\beta$-lactoglobulin and ovalbumin in random samples of human milk. Mothers who did not plan to breast feed were randomly given either cow-milk formula (Enfalac ${ }^{\circledR}, \mathrm{n}=40$ ), soy formula (Prosobee ${ }^{\circledR}, \mathrm{n}=41$ ), or casein-hydrolysate formula (Nutramigen $\AA, n=43$ ), which they were told to feed the infant for at least 6 months. The mothers and examiners were unaware of the formula type. Infants were examined by a physician at 2, 4,6,12, and 18 months, and an eczema score was constructed based on affected parts of the body, type of eruption, and severity. Student $t$-test was used to compare squareroot transformed eczema scores, and proportions were compared using the $\chi^{2}$ test with Yates correction. The three formula groups were compared using the Tukey W-test for transformed scores and $\chi^{2}$ test for proportions.

Among breast-fed infants, maternal dietary restriction was associated with a lower mean eczema score and a lower proportion of affected infants [not further discussed for the purposes of this study]. A comparison of the three formula-fed groups shows a lower proportion of affected infants and lower eczema scores in the group given the casein hydrolysate than in the other two groups. Only nine infants in the casein-hydrolysate group developed eczema, whereas 36 infants in the soy group and 38 infants in the cow-milk group developed eczema. No difference was detected in the incidence of eczema between the soy formula and cow-milk formula groups. The authors recommended that formula-fed infants with family history of atopy be given a casein hydrolysate formula.

Strengths/Weaknesses: Strengths include use of a homogenous group with positive family history of atopic disease, random assignment to one of three formula groups, one of which was restricted to soy formula for at least 6 months, randomization after maternal selection of breast or bottle, blinding of mothers and observers to formula type, and separate analysis of the formula group due to self-selection. Weaknesses include the relatively small sample size of soy formula-fed infants $(\mathrm{n}=41)$ and the lack of mention of introduction of other foods. There were no multivariate analyses, and although baseline characteristics were said not to differ, no statistical comparisons were made.

Utility (Adequacy) for CERHR Evaluation Process: This study is useful and shows no apparent benefit of soy formula over cow-milk formula in a high risk population.

Chandra and Hamed (1991) and Chandra et al. (1989b), supported by the National Health Research Development Program of Health and Welfare Canada, Carnation Nutritional Products, and the Nutrition Research Education Foundation, conducted a prospective randomized controlled study to examine the effects of exclusive breast feeding and the feeding of one of three different types of formula on the incidence of atopic disease in predisposed infants. Mothers were recruited if either they or the baby's father had a family history of atopy. Mothers planning to breast feed $(n=60)$ were instructed to breast feed exclusively but were not given any other instructions regarding their own diets. Mothers who did not plan to breast 
feed were randomly given either cow-milk formula (Similac $®, n=24$ ), soy formula (Isomil ${ }^{\circledR}$, $n=25$ ), or whey-hydrolysate formula (Good Start H.A. $\left.{ }^{\circledR}, n=68\right)$, which they were told to feed their infants exclusively for 6 months. The mothers and examiners were unaware of formula assignment. After 6 months, families were given usual advice for the feeding of infants with family history of allergy. Infants were examined at 6, 12, and 18 months of age for clinical manifestations of possible allergy. Skin tests were conducted on symptomatic infants using commercial antigens, and IgE antibodies to milk and soy were measured using commercial kits.

Infants in the whey-hydrolysate group had significantly lower incidence of atopic symptoms than those in the cow-milk and soy-formula groups. No significant differences were apparent between the cow-milk and soy-formula groups or between the whey-hydrolysate and breastfed groups. Serum IgE antibodies to cow-milk or soy proteins were described as more common in infants fed the respective protein source [statistical comparisons not reported]. Skin prick tests in symptomatic infants did not identify significant differences between cow milk- and soy formula-fed infants. The authors concluded that there was a lower incidence of atopy in predisposed infants who were breast-fed or fed hydrolysate compared to predisposed infants fed cow-milk or soy formula.

Strengths/Weaknesses: Strengths include use of a homogeneous group with positive family history and randomization to formula groups after the maternal decision not to breast feed. The use of three formula groups with exclusive feeding for 6 months and the blinding of mothers and observers to formula type are additional strengths. The sample sizes were moderate with only 25 subjects in the soy-formula group. Breast feeding mothers were self-selected. There was no mention of solid foods, and although baseline characteristics were said to not differ, there were no multivariate analyses.

Utility (Adequacy) for CERHR Evaluation Process: This study is useful and shows no apparent benefit of soy formula over cow-milk formula in a high-risk population.

Chandra $(1997,1998)$ presented 5-year results from the above study. The prevalence of eczema by 60 months was $6 / 60$ [10\%] in the breast-fed group, 10/68 [15\%] in the wheyhydrolysate group, 19/68 [28\%] in the soy-formula group, and 20/67 [30\%] in the cow-milk formula group. The prevalence of asthma by 60 months was 4/60 [7\%] in the breast-fed group, $7 / 68$ [10\%] in the whey-hydrolysate group, 14/68 [21\%] in the soy formula group, and 16/67 [24\%] in the cow-milk formula group. Skin prick testing and double-blind placebo-controlled food challenges showed a lower incidence of food allergy in the whey-hydrolysate and breastfed groups. The authors concluded that breast feeding is preferred in children with a high risk of atropy, and that among formulas, the whey-hydrolysate formula was preferred to cow-milk or soy formula. [The Expert Panel notes that the whey-hydrolysate formula was marketed by Carnation, a sponsor of this study.]

Strengths/Weaknesses: This 5-year follow-up has the same strengths and weaknesses as the earlier reports (Chandra et al., 1989b; Chandra and Hamed, 1991).

Utility (Adequacy) for CERHR Evaluation Process: This study is useful and shows no apparent benefit of soy formula over cow-milk formula in a high-risk population.

Klemola et al. (2002), supported by Turku University Hospital, the Social Insurance Institution, and Valio Ltd., evaluated 170 children with cow milk allergy for the development of allergic symptoms on soy formula or extensively hydrolyzed formula. The children were diagnosed with cow milk allergy at age 2-11 months using a double-blind placebo controlled food challenge. Children were randomly assigned by center and age to extensively hydrolyzed 
formula $(n=90)$ or soy formula $(n=80)$ until age 2 years. [Both formulas were manufactured by Valio Ltd.] Other sources of soy protein, egg, fish, citrus, peanuts, peas, tomatoes, strawberries, and chocolate were avoided for the first year. When the study formula was suspected of causing symptoms, a double-blind food challenge was carried out using both formulas, and the child was switched to the opposite study formula. If both formulas were believed to cause symptoms, the child was put on an amino acid formula. At the time of diagnosis of cow milk allergy and at ages 12 and 24 months, children underwent skin testing and specific IgE antibody measurement. Skin testing was carried out with dried cow-milk formula, soy formula, the extensively hydrolyzed formula, a negative control, and a positive control (histamine). Statistical comparison of the incidence of adverse reactions to each study formula with $95 \%$ CI was carried out using an exact binomial distribution. For categorical variables, $\chi^{2}$ testing was used.

Parents suspected adverse reaction in $28 \%$ of subjects on soy formula and $11 \%$ of subjects on extensively hydrolyzed formula (relative risk $[\mathrm{RR}]=2.48[\mathbf{9 5 \%} \mathbf{C I}=\mathbf{1 . 2 8 - 5 . 1 2}] ; P=0.006$ ). Of the adverse reactions that were followed by a controlled food challenge, $10 \%$ were positive in the soy formula group and $2.2 \%$ were positive in the extensively hydrolyzed formula group $(\mathrm{RR}=4.50[\mathbf{9 5 \%} \mathbf{C I}=\mathbf{1 . 1 2 - 2 8 . 0 0}], P=0.031)$. Some of the reactions during the challenge were characterized as doubtful and most reactions were not supported by IgE and skin test data. During the 2-year follow-up period, a similar number of children assigned to each formula had $\mathrm{IgE}$ antibodies to soy protein. Among children younger than 6 months who were assigned to soy formula, $40 \%$ were suspected by parents of adverse reactions. [Data in 6-month-old children were not reported for the comparison formula.] The authors concluded that more than $70 \%$ of infants with cow milk allergy tolerated soy formula and that IgE-mediated reactions to soy formula were unusual. They indicated that soy formula may be considered a first choice for cow milk intolerant children with the possible exception of children younger than 6 months.

Strengths/Weaknesses: Randomization to two different formulas and the adequate sample size are strengths of this study. Weaknesses are the lack of clear criteria for what constituted an adverse response and the failure of laboratory testing to confirm many of the presumed hypersensitivity reactions. In addition, because the study extended to age 2 years, there would have been different lengths of follow-up for the children from as little as 13 months to as much as 22 months.

Utility (Adequacy) for CERHR Evaluation Process: This study is of marginal utility in the evaluation process.

Odze et al. (1993), funding not indicated, reported biopsy findings from 20 infants with allergic proctocolitis. The authors evaluated a possible association between numbers of eosinophils in colonic mucosal biopsy specimens and infant food source. The diagnosis of allergic proctocolitis was based on the presence of rectal bleeding in infants younger than 1 year who responded to a change of diet and in whom no other cause of rectal bleeding was found. Of the 20 infants, seven had been fed human milk, five cow-milk formula, five soy formula, and three a combination. Cow milk-fed infants presented at a mean age of 21 days, soy formula-fed infants at 36 days, breast-fed infants at 85 days, and combination infants at 144 days. These differences were not significant. The signs and symptoms of proctocolitis were similar in the different feeding groups. No significant relationship was found between the number of eosinophils in the mucosa and infant diet.

Strengths/Weaknesses: A strength of this case series is the use of biopsies with quantification of eosinophils. 
Utility (Adequacy) for CERHR Evaluation Process: This study is not useful in the evaluation process.

Lack et al. (2003), supported by the Medical Research Council, the Wellcome Trust, and several U.K. government agencies, used data from the Avon Longitudinal Study of Parents and Children to determine what factors, including soy intake, affected development of peanut allergy. From a large prospective cohort study of 13,971 preschool-aged children, 49 children with peanut allergy were identified and compared to controls using data retrospectively collected by masked telephone interview. One control group of 70 atopic children was randomly selected from those who were reported to have eczema or whose mothers had a history of eczema. A second control group consisted of 140 randomly selected children who did not have peanut allergy. The telephone survey of case and control parents was designed to collect additional information on maternal peanut consumption during pregnancy and lactation, family history of peanut allergy, and use of peanut oil-containing skin lotions and creams. Of 49 children identified, 36 underwent skin testing and 29 tested positive for peanut allergy. There was a statistically significant association between soy product consumption and both peanut allergy and positive peanut challenge. Soy-milk or soy-formula consumption in the first 2 years occurred in $8.3 \%$ of the children in the total cohort, compared to $24.5 \%$ with peanut allergy and $34.8 \%$ with positive peanut challenge. In addition, 9 of 10 children for whom consumption history was available were exposed to soy before peanut reaction. Step-wise logistic regression analysis, controlling for significant risk factors (rash over joints and in skin creases and oozing, crusted rash), confirmed that early soy consumption was an independent risk factor for peanut allergy $(\mathrm{OR}=2.61,95 \% \mathrm{CI}=1.31-5.20)$ and positive peanut challenge $(\mathrm{OR}=3.15,95 \% \mathrm{CI}=1.27-7.80)$.

The possibility that peanut allergy was associated with underlying cow-milk allergy rather than with soy product intake was examined, but cow-milk allergy was determined not to be a confounder. Only four of 289 children in the cohort who were allergic to milk also had peanut allergy. The authors speculated that although no child in the study reacted to both peanuts and soy products, an immunologic co-reactivity between peanuts and soybeans could have caused cross-sensitization without clinical soy product allergy. Alternatively, the authors noted that an unidentified confounding factor could have been responsible for the observed relationship.

Strengths/Weaknesses: This nested case control study within a large prospective cohort study included two comparison groups, one "diseased" (atopic children) and one normal. It seems that soy-product feeding data were collected prospectively with respect to outcome. There were appropriate multivariate analyses. The reasons for soy product consumption in the first place could have confounded the association between soybean and peanut allergy. This possibility was likely addressed by the atopic control group, but the reported comparisons seem to have been conducted with both control groups combined.

Utility (Adequacy) for CERHR Evaluation Process: This study is useful for the evaluation process.

Klemola et al. (2005), funded by the Finnish Social Insurance Institution, Turku Hospital, and Valio, Ltd., carried out a prospective, randomized study of an extensively hydrolyzed whey formula (PeptidiTutteli ${ }^{\circledR} ; \mathrm{n}=90$ at study onset, $\mathrm{n}=76$ after drop-outs) or soy formula (Soija Tutteli ${ }^{\circledR} ; \mathrm{n}=80$ at study onset; $\mathrm{n}=72$ after drop-outs) in infants with cow milk allergy. [Both formulas were made by the corporate sponsor of the study.] Infants were diagnosed with cow milk allergy based on double-blind food challenge at a mean age of 7 months (range $=2$ 11 months). Infants were given no other source of soy protein during the first year, and other specific foods were avoided if skin testing suggested sensitivity. Infants were examined at age 2,3 , and 4 years. Serum IgE levels were measured in infants at randomization, and at age 1, 
2 , or at 4 years [not all children were evaluated at all time points]. Parents were questioned at age 18 months, and 2, 3, and 4 years about food avoidance and adverse reactions to soy or peanuts. Pearson correlations, $\chi^{2}$, and multivariate logistic regression was used to analyze the data.

Detectable soy IgE levels were numerically more prevalent in the soy formula-fed infants at all ages, but the difference from the hydrolysate-fed group was never statistically significant. At 4 years, the OR $(95 \% \mathrm{CI})$ for soy IgE positivity among soy formula-fed infants was 2.28 $(0.90-5.76 ; P=0.082)$. No difference was detected between the groups in prevalence of peanut $\mathrm{IgE}$ positivity $(\mathrm{OR}=1.27,95 \% \mathrm{CI}=0.35-4.71 ; P=0.717)$. None of the 4-year-old children were reported to have had an immediate reaction to soy products, although one child in the soy formula-fed group had a delayed reaction (eczema). No difference was detected between the groups in the proportion of children with reported reactions to peanuts. The authors concluded that feeding soy formula to children with cow milk allergy may slightly increase the risk of sensitization at age 4, but that clinically important reactions to soy products were uncommon. They also concluded that feeding soy formula did not increase the risk of sensitization to peanuts.

Strengths/Weaknesses: Strengths include the randomized design with long-term follow-up to 4 years of age and the biological measures of sensitization. However, the reasons for the 22 dropouts were not given. With respect to adverse reactions to peanuts, it unclear how many children in the sample were really exposed to peanut products; it seems that approximately one-third did not think they ever had been exposed. If cross-reactivity is the reason for the high correlation between IgE antibodies to soy and to peanuts, it is difficult to understand why it would be expected that use of soy formula would increase the risk of peanut-specific IgE.

Utility (Adequacy) for CERHR Evaluation Process: This study is somewhat useful in the evaluation process.

Zoppi et al. (1979), funding not indicated, evaluated full-term infants given soy formula for the first 4 months of life. Three different protein levels were used $(2.5,4.0$, and $5.5 \mathrm{~g} / \mathrm{kg} / \mathrm{day}$ [kg bw or kg formula was not specified; assignment method was unclear]). There were 39 infants in the study, three of whom were hospitalized for illness and were not included in the results. Diet was adjusted every 2 weeks based on infant body weight. Blood samples were taken early in the fifth study month for determination of hemoglobin, red blood cell count, protein electrophoresis, immunoglobulins, urea nitrogen, creatinine, glucose, electrolytes, acid-base status, and amino acids. Data were compared using Student $t$-test.

No significant differences in gains in weight and length over the 4-month observation period were detected between groups. Hematologic parameters were within the normal range and no differences between groups were detected. Concentrations of urea nitrogen reflected the different nitrogen intakes. Serum protein concentration was described as slightly higher in the high-protein group, although not significantly different from the other 2 groups. Serum albumin and $\alpha$ - and $\beta$-globulins were similar between groups. $\gamma$-Globulins increased with increasing protein intake. An increase in immunoglobulin concentration with increasing protein level was also described; however, due to the large SD, only differences in IgG were significant. The authors noted that the serum levels of $\gamma$-globulin and immunoglobulins in the infants on the high-protein diet were similar to those reported in a different study in infants fed $<50 \%$ this amount of cow-milk protein. The authors also reported that morbidity was higher in the lowprotein group than in the other two groups, with the low-, mid-, and high-protein groups having $3.18,2.13$, and 2.00 infections per infant, respectively. 
Strengths/Weaknesses: It is a strength that blood measures after 4 months of feeding were associated with the level of soy protein. Infections were not well described, and no significance testing was used. The sample size was small.

Utility (Adequacy) for CERHR Evaluation Process: This study is somewhat useful in the evaluation process.

Zoppi et al. (1982), supported by "CNR and MPI grants," compared immunocompetence in infants fed formulas containing either cow-milk protein or soy protein. Infants were assigned to one of four feeding groups, as shown in Table 25 [method of assignment unclear; previous study cited]. Two different protein concentrations were obtained for each formula by diluting with water and adding dextro maltose to maintain caloric content. The infants received their assigned formulas from birth to 4.5 months and were examined every 2 weeks for weight, length, and illness (chiefly infection). After 4.5 months, blood samples were evaluated for hemoglobin, hematocrit, white blood cell count, protein electrophoresis, urea nitrogen, creatinine, glucose, electrolytes, iron, transferrin, cholesterol, and triglycerides.

Immunoglobulin, complement, and amino acid determinations were carried out. Tests of immune function were carried out on isolated monocytes and included rosette formation to sheep erythrocytes, estimation of surface immunoglobulins, and mitogen response to phytohemagglutinin, pokeweed mitogen, concanavalin A, and Staphylococcus protein A. Data were analyzed using the 2-sample $t$-test or the Behrens-Fisher test and multivariate linear discriminant analysis.

No differences in head circumference and mean gains in weight and length were detected between the groups. There were more episodes of infection in infants fed the low-protein soy formula (3.4 episodes per infant) than in infants in the other groups (1.8-2.6 episodes per infant [significance levels not given]). Hematologic parameters fell within normal ranges for all groups; although in comparison to other groups, the authors noted a significantly lower hematocrit in the low-protein soy group, a higher white blood cell count in the high-protein soy group, and higher iron concentration (with lower transferrin values) in infants fed either soy formula. Serum cholesterol and triglycerides were lower in the two soy groups. [Levels of significance were not given for these comparisons.]

Total serum protein, albumin, and $\beta$-globulin, and $\gamma$-globulin levels were described as higher in the cow-milk than soy groups, although the differences were not significant. Serum gammaglobulins, IgA, IgG, and IgM were significantly lower in the low-protein soy group than in the other groups. Complement $\mathrm{C} 3$ levels were lower in soy formula-fed infants than in those receiving cow milk. Complement $\mathrm{C} 1$ and $\mathrm{C} 3$ levels were lower in the two low-protein groups compared to the two high-protein groups. No statistically significant differences were detected between groups in monocyte function, although the authors reported a "tendency" for T cells from infants in high-protein groups to show a greater response to mitogens. Discriminant analysis incorporating $\gamma$-globulins, transferrin, complement $(\mathrm{C} 4$ and $\mathrm{C} 1)$, activated rosette formation, and mitogenic response showed clear separation of groups by protein amount and source (cow milk or soy). Using only T-cell function parameters, a separation between groups was evident with a comparison of low-protein soy and high-protein cow-milk groups and, to a lesser extent, between low-and high-protein cow-milk groups. The study authors concluded that soy protein was of lower nutritional value than cow-milk protein, and that low-protein formulas were suboptimal with respect to immune function.

Strengths/Weaknesses: The strengths and weaknesses of this study are similar to those of the previous study (Zoppi et al., 1982). 
Utility (Adequacy) for CERHR Evaluation Process: This study is somewhat useful in the evaluation process.

Zoppi et al. (1983), funding not indicated, studied the relationship between formula type and antibody response to vaccination in 62 healthy infants born at term. The study included 27 infants whose mothers decided to breast feed. Other study infants were randomly assigned to a high-protein cow-milk formula $(4.4 \mathrm{~g} / \mathrm{kg} / \mathrm{day} ; \mathrm{n}=7)$, a low-protein cow-milk formula (1.6 $\mathrm{g} / \mathrm{kg} / \mathrm{day} ; \mathrm{n}=9$ ), an adapted cow-milk formula with a casein/albumin ratio of 40:60 (protein $1.8 \mathrm{~g} / \mathrm{kg} / \mathrm{day} ; \mathrm{n}=10$ ), or a soy formula (protein $4.6 \mathrm{~g} / \mathrm{kg} / \mathrm{day} ; \mathrm{n}=9$ ). Infants received their assigned formula exclusively until 5 months of age when they were all switched to the same diet (with $\sim 3.5 \mathrm{~g} / \mathrm{kg} / \mathrm{day}$ of protein) and followed to 1 year of age. [The Expert Panel assumes the per kg designations are per kg bw.]

Weight and length were measured every 2 weeks. Infants were vaccinated against poliovirus, diphtheria, tetanus, and pertussis at 2 and 4 months of age. Blood was sampled at 5 and 8 months of age and analyzed for antibodies, immunoglobulins, hemoglobin, hematocrit, white blood cell count, protein electrophoresis, urea nitrogen, creatinine, glucose, electrolytes, iron, transferrin, cholesterol, and triglycerides. Data were evaluated using ANOVA [post-hoc tests not designated except as orthogonal].

All groups had normal hematologic variables, blood urea nitrogen, amino acid levels, serum protein, immunoglobulin, zinc, transferrin, and mean gains in weight, height, and head circumference. Groups differed in antibody response to vaccination. Breast-fed infants and infants fed the high-protein cow-milk formula had protective antibody levels at age 5 and 8 months. Antibody levels in infants fed the adapted formula were significantly higher at 5 months but lower at 8 months than those of breast-fed infants. In infants fed the low-protein cow-milk or soy formulas, antibody levels were significantly lower than in breast-fed infants. Infants fed soy formula had significantly lower levels of antibodies to tetanus, diphtheria, and pertussis than did the breast-fed group or the high-protein cow-milk group. However, infants fed soy, low-protein cow-milk, and adapted cow-milk formulas had significantly higher levels of antibodies to poliovirus Type 2 than did breast-fed infants. The mean number of episodes of infection per infant was highest in the soy formula-fed group (2.9), compared to breast-fed (0.5), high-protein cow milk (1.6), low-protein cow milk (2.7), and adjusted formula (2.4) [statistical significance not reported]. The authors concluded that soy formula-fed infants had an impairment of antibody response to common viral and bacterial vaccinations, with the exception of poliovirus Type 2 . They suggested that recommended protein intakes should be re-assessed to take into account both the quality and quantity of dietary protein. They recommended an animal protein intake of $3 \mathrm{~g} / \mathrm{kg}$ bw/day and concluded that vegetable protein should not be given to infants during the first months of life.

Strengths/Weaknesses: Strengths included a concrete measure of immune function in infants, vaccination response, and the use of four feeding groups with random assignment after breast feeding-preference selection. Weaknesses included the small numbers, although significant differences were still identified on some measures, the possible selection bias for breast-fed mothers, and the lack of demonstrated clinical significance of the observed differences in immune response.

Utility (Adequacy) for CERHR Evaluation Process: This study is useful for the evaluation process.

May et al. (1982), supported by the Public Health Service and the National Dairy Council, measured serum antibodies to five major milk proteins and a soy-protein isolate in 67 infants fed one of two cow-milk preparations, a soy product (EdiPro A), or a mixture of $73 \%$ cow 
milk/27\% soy isolate from birth to 112 days of age. The infants were then switched to another product. Serum samples were obtained at 28, 112, 140, 168, and 196 days of age. Samples were also obtained from seven infants who were breast-fed from birth to 112 days. Additional data were obtained from 60 older children up to 16 years of age from a separate food-sensitivity study in which subjects received unrestricted diets including cow milk. The serum antigen binding capacity (total antibody content) was determined using radiolabeled antigens. Data were analyzed using the Student $t$-test. After 112 days, binding of soy protein in the serum of soy formula-fed infants was significantly less than binding of milk proteins by infants fed only cow milk. Feeding a soy product from birth resulted in a comparable or greater antibody response to subsequent cow-milk introduction than was seen in infants fed cow milk from birth. In light of this finding and a decreased antibody response to heat-treated milk, the authors suggest that heat-treated cow-milk formula rather than soy formula may be a preferred substitute for human milk.

Strengths/Weaknesses: There were four feeding groups with defined exposures, and there was some information about solid food intake. It is a weakness that the data were not originally collected for this study but rather were from different sites with different initial protocols. The group sizes were small.

Utility (Adequacy) for CERHR Evaluation Process: This study is somewhat useful for the evaluation process.

McDonald et al. (1984), funding not indicated, conducted a study to evaluate immunologic mechanisms involved in food protein-induced enterocolitis. Eighteen infants (14 male) presented with vomiting and diarrhea that continued after switching to soy formula and had stools containing mucus, blood, and leukocytes. Symptoms disappeared within 2-3 days of removing whole protein from the diet. Class-specific antibodies to ovalbumin, soy, and cow milk were measured before food challenges to obtain baseline levels. Infants were then challenged with sequential oral $0.6 \mathrm{~g}$ protein $/ \mathrm{kg}$ bw doses of egg white, soy formula, and skim milk. Forty-eight hours or 5 days were allowed for re-attainment of baseline values after either a negative or positive challenge, respectively.

Infants with positive challenges to soy formula and egg had significantly higher IgG antisoybean antibody concentrations than those with negative responses. Similarly, children with positive challenges to soy formula and egg also had higher IgA food-antibody titers. IgM levels did not differ between positive and negative challenge responders. However, in 12 infants who were followed after a single soy formula challenge, IgM anti-soybean antibody was found to increase with a negative response and decrease with a positive response. The authors stated that these findings suggested altered immunologic responses to ingested antigens in infants having food protein-induced enterocolitis.

Strengths/Weaknesses: The study included a well defined group with confirmed disease and looked at a range of antibodies. It is a strength that baseline, challenge, and post-challenge values were included. Weaknesses include the small sample size, the unknown or poorly described feeding histories, and the broad subject age range.

Utility (Adequacy) for CERHR Evaluation Process: This study is somewhat useful for the evaluation process.

Jenkins et al. (1984), funding not indicated, studied 46 children with colitis diagnosed within a 4-year period. They reported that the age distribution of the children showed peaks at $<2$ years and 6-13 years, with all 38 children in the older group suffering from either ulcerative colitis or Crohn's disease. The researchers compared the remaining eight children with selected 
controls from the older group. Of these eight subjects, six presented before 4 months and were switched from a cow-milk to a soy-milk diet. The remaining two subjects were receiving a mixed diet and were switched to an egg-free cow-milk diet. For each of these eight patients and selected controls, dietary history, family history of allergy, blood count, serum immunoglobulins, IgE, and IgE antibodies to whole cow milk, $\beta$-lactoglobulin, and ovalbumin were studied. Colonoscopies were also carried out in seven of the patients and in the controls. Biopsies were taken before and after the implementation of the exclusion diets and examined for eosinophils and IgE-containing cells.

Symptoms disappeared in all eight patients when switched to soy formula. Symptoms recurred within 2-6 weeks of the switch in three of eight patients but resolved after a chicken-based formula was substituted. Two patients who were given cow milk again 9-12 months after diagnosis showed symptoms again within $24 \mathrm{hr}$. Another patient who was given beef broth at 1 year also showed symptoms. All symptoms resolved after return to the exclusion diet. The researchers found that positive family history was significantly more common in the eight patients with food-allergy colitis than in the selected controls. Blood eosinophil counts, serum IgE concentrations, and antibodies were also higher in the study group. Colonoscopies of the study group were distinctly different from those of controls, with an increase in eosinophils and IgE-containing cells. The authors concluded that colitis arising during infancy was most likely a result of food allergy and was best treated with an exclusion diet.

Strengths/Weaknesses: It is a strength that biopsies were taken before and after exclusion diets, but a weakness that infants were compared with much older "selected" controls.

Utility (Adequacy)for CERHR Evaluation Process: This study is not useful for the evaluation process.

Fort et al. (1990), funding not indicated, conducted a retrospective study of 59 children with autoimmune thyroid disease, their 76 healthy siblings, and 54 healthy, unrelated control children to determine whether feeding soy formula or breast feeding was associated with the development of thyroid disorders. The children with autoimmune thyroid disease included 52 with Hashimoto's thyroiditis and seven with Graves' disease. A nutritionist evaluated the history of infant feeding early in life, including the type of formula given and the age at which solid food was introduced. Proportions were evaluated using $\chi^{2}$ test. No significant differences were seen in the prevalence or duration of breast feeding or in the age at which solid foods were introduced. Among children with thyroid disease, there were no significant differences in the initial serum values of thyroxine or thyroid-stimulating hormone or in anti-thyroid antibody titers between breast-fed and bottle-fed infants [data not shown]. Of children with thyroid disease, $31 \%$ had received soy formula as infants compared to $12 \%$ of healthy siblings $(P<0.01)$ and $13 \%$ of healthy unrelated controls $(P<0.02)$. The authors concluded that they were unable to document an advantage of breast feeding with regard to the subsequent development of autoimmune thyroid disease, The greater prevalence of soy-feeding among children with thyroid disease compared to children without thyroid disease was proposed as being due to a possible decrease in cow-milk tolerance among children predisposed to developing thyroid disease or to possible adverse effects of soy on the developing thyroid.

Strengths/Weaknesses: The use of sibling controls plus a healthy unrelated control group is a strength. Weaknesses include the retrospective data collection and the small sample. As the authors suggested, the reasons for selecting soy formula may have confounded the association between this product and subsequent thyroid disease. This study cannot make that distinction.

Utility (Adequacy) for CERHR Evaluation Process: This study is useful for the evaluation process. 
Ostrom et al. (2002), supported by the Ross Products Division, Abbott Laboratories, conducted a feeding trial to evaluate the immune status and morbidity of infants fed soy proteinisolate formulas with and without added nucleotides. Infants of mothers who decided not to breast feed before enrollment were randomly assigned to receive soy formula $(n=92)$ or soy formula with monomeric nucleotides added at $74 \mathrm{mg} / \mathrm{L}(\mathrm{n}=94)$ exclusively, from enrollment to 4 months. Both of these formulas, based on Isomil ${ }^{\circledR}$, contained nucleotides $300 \mathrm{mg} / \mathrm{L}$ before supplementation. A third non-randomized group of 81 infants received human milk exclusively for up to at least 2 months and then received cow-milk formula supplements (Similac ${ }^{\circledR}$ with Iron; total nucleotides, $7 \mathrm{mg} / \mathrm{L}$ ). Recommended immunizations were administered at 2, 4, and 6 months according to the 1994 immunization schedule of the American Academy of Pediatrics. Immune status of the infants was assessed by comparing morbidity and antibody responses to specific immunizations. Blood samples were taken at 6,7, and 12 months of age. Antigen-specific immune responses, immune cell populations, and serum $\operatorname{IgG}$ and $\operatorname{Ig} A$ were measured. ANOVA and ANCOVA models with the Tukey-Kramer method were used to compare continuous data from the three groups, controlling for site. Proportions of children with diarrhea, otitis media, and antibiotic usage were analyzed using $\chi^{2}$ test.

No significant differences were detected between the three groups at 6, 7, or 12 months of age in total serum IgG or IgA concentrations or in serum concentrations of antibodies against poliovirus $(\operatorname{Ig} A)$, diphtheria $(\operatorname{IgG})$, or tetanus $(\operatorname{IgG})$. Although no significant differences were detected in response to Hemophilus influenza $\mathrm{b}$ between infants fed soy formula with or without added nucleotides, infants fed nucleotide-supplemented soy formula had significantly higher geometric mean levels of antibody to Hemophilus influenza $\mathrm{b}$ at 7 and 12 months than did infants fed human milk. Although diarrhea reported by parents did not differ by feeding group, physician-reported diarrhea was significantly less frequent in the human milk-fed group compared to both soy-formula groups. No feeding-group differences for otitis media or antibiotic usage were detected.

The authors concluded that infants fed soy-based formulas have normal immunoglobulin levels, normal responses to vaccine antigens, similar morbidity associated with otitis media, and thus normal immune development compared with breast-fed infants. They also concluded that nucleotide supplementation of soy formula provided no benefit other than a marginal benefit in response to Hemophilus influenza $\mathrm{b}$ immunization.

Strengths/Weaknesses: Strengths include random assignment of infants whose mothers did not choose breast feeding, randomization within site, blinding of assignment, and the block design accounting for infant sex. The sample size was relatively large and an appropriate repeated-measures analysis was used for vaccine response data. Weaknesses include the varying lengths of time for which breast-fed infants were fed exclusively by that method and the lack of baseline immune function data before vaccination. There could have been selection bias in women who elected to breast feed.

Utility (Adequacy) for CERHR Evaluation Process: This study is useful for the evaluation process.

Cordle et al. (2002), supported by the Ross Products Division, Abbott Laboratories, reported additional results from the trial reported by Ostrom et al. (2002). No feeding-group differences were detected in total white blood cell count or in numbers or percentages of the five major leukocyte populations; however, at 6 months, the breast-fed group had significantly higher numbers and percentages of eosinophils than the group given nucleotide-supplemented soy formula. The breast-fed group also had a higher percentage of lymphocytes at 12 months than the group given nucleotide-supplemented soy formula. No feeding-group differences were detected in numbers or percentages of B, pre-B, or mature B lymphocytes. No significant 
differences were identified in the numbers or percentages of total T, helper T, or cytotoxic/ suppressor $\mathrm{T}$ cells between the breast-fed group and the group given unsupplemented-soy formula. At 7 months, the group given supplemented-soy formula had a higher percentage of total T lymphocytes, higher numbers and percentages of helper $\mathrm{T}$ lymphocytes, and higher percentages of non-natural killer-like T cells. Despite the differences, all values were reportedly within normal ranges.

The researchers were unable to detect feeding-group differences in expression of the human leukocyte antigen or in the maturation state of helper T lymphocytes, with the exception that the supplemented-soy group had higher percentages of naive helper T cells than the breast-fed group at 6 and 7 months and a higher number of memory/effector helper $T$ cells than the breastfed group at 7 months. The study authors reported that the concomitant increase in CD45RA and CD45RO cells suggested a balanced, antigen-driven conversion of the helper $\mathrm{T}$ cell population. No differences were detected in numbers or percentages of natural killer lymphocytes or natural killer $\mathrm{T}$ lymphocytes between the three feeding groups, with the exception of the supplemented soy group having lower numbers and percentages of natural killer cells compared with the breast-fed group and the unsupplemented soy group having lower percentages of natural killer $\mathrm{T}$ cells than the breast-fed group at 12 months.

The authors concluded that there were no consistent significant differences in immune status, maturation, or level of immunocompetence between infants fed a soy-based formula and those fed human or cow milk. They also reported that their study failed to demonstrate a strong effect of nucleotide supplementation of soy formula on immune status of infants.

Strengths/Weaknesses: Strengths and weaknesses are similar to those for Ostrom et al. (2002).

Utility (Adequacy) for CERHR Evaluation Process: This study is useful for the evaluation process.

3.1.2.4 Thyroid function: Shepard (1960), supported by the Platex Park Research Institution, reported three children with goiter related to the feeding of soy formula. The infants were clinically euthyroid but had low-normal levels of protein-bound iodine and high ${ }^{131}$ I uptakes. When soy formula was discontinued, ${ }^{131}$ I uptake returned to normal. In two of the cases, the goiter decreased in size or disappeared with the discontinuation of soy formula. Reduction in goiter size occurred in the third case when iodine was added to the diet and the brand of soy formula was changed. The authors concluded that lack of iodine was the cause of the soy formula-associated goiter.

Strengths/Weaknesses: These cases involve a special population of infants with congenital hypothyroidism. The case series format is a weakness.

Utility (Adequacy) for CERHR Evaluation Process: This study is useful for the evaluation process.

Chorazy et al. (1995), support not indicated, presented a case report of a child with congenital hypothyroidism who was placed on soy formula at birth due to parental concern about a family history of milk allergy. The child's thyroid-stimulating hormone could not be suppressed into the normal range with oral thyroxine supplementation until cow-milk formula was substituted for soy formula. Stool frequency decreased from 5-7/day on soy formula to 2-3/day on cow milk formula. The authors believed this study and a similar study from 1965 provided evidence that soy formula interferes with thyroxine absorption from the intestine through fecal wastage. They noted that although iodine supplementation of formulas and a reduction in the fiber 
content of soy formulas decreased the risk of thyroxine malabsorption, formula intolerance might have led to an increase in stool frequency and a risk of fecal wasting of thyroxine in their patient.

Strengths/Weaknesses: This case involves a special population of infants with congenital hypothyroidism. The case report format is a weakness.

Utility (Adequacy) for CERHR Evaluation Process: This study is useful for the evaluation process.

Jabbar et al. (1997), funding not indicated, reported three infants with congenital hypothyroidism who experienced apparent malabsorption of thyroxine while on soy formula. Two of the infants were found on laboratory monitoring to have elevated thyroxine levels after they had been switched from soy to cow-milk formula. The thyroxine levels normalized with the thyroid replacement dose was decreased. The third infant did not suppress thyroidstimulating hormone levels on thyroxine replacement doses as high as $19 \mu \mathrm{g} / \mathrm{kg}$ bw/day. Switching from soy formula to cow-milk formula resulted in normalization of thyroidstimulating hormone with an administered thyroxine dose of $14 \mu \mathrm{g} / \mathrm{kg}$ bw/day. The authors concluded that soy formula could result in decreased absorption of administered thyroxine and decreased enterohepatic reabsorption of thyroxine with consequent fecal wastage.

Strengths/Weaknesses: These cases involve a special population of infants with congenital hypothyroidism. The case series format is a weakness.

Utility (Adequacy) for CERHR Evaluation Process: This study is useful for the evaluation process.

Mousavi et al. (2005), support not indicated, reported a case-control study of goiter in school girls. There were 284 girls aged 7-12 years who were found on routine examination to have a goiter. Among girls with a normal thyroid examination, 288 aged 6-12 were randomly selected as controls. Mothers were given a questionnaire that included information on diet within the previous month, among other potential risk factors for goiter. Pearson's $\chi^{2}$ test was used for univariate analysis followed by multiple logistic regression. A similar proportion of each group reported soy product exposure ( $43.7 \%$ of cases and $45.5 \%$ of controls). The authors identified the method of home storage of iodized salt to be the only significant difference between the cases and controls.

Strengths/Weaknesses: The authors used a good method for subject selection of cases and controls. There was, however, only limited information about soy exposure consisting of a maternal study of "any" use in the previous month. This exposure information may be particularly problematic in this community, where it seems that nearly half the population eats soy products.

Utility (Adequacy) for CERHR Evaluation Process: This study is somewhat useful in the evaluation process.

3.1.2.5 Reproductive endpoints: Freni-Titulaer et al. (1986), funding not indicated, conducted a case-control study to evaluate factors associated with a three-fold increase in the number of reported patients with premature thelarche between 1978-1981 in Puerto Rico. Potential cases included girls between the ages of 6 months and 8 years with palpable breast tissue at least $1.5 \mathrm{~cm}$ in diameter at the time of diagnosis of premature thelarche in the absence of other evidence of premature sexual development. Of 552 potentially eligible subjects diagnosed between 1978-1982 with premature thelarche, 397 parents returned consent forms, 
and 130 representative case subjects were systematically selected for the study. An agematched control subject was selected for each case subject, including where possible, a friend of the case. Parents were interviewed to determine family history and possible exposures. Data were analyzed by the computation of OR with $95 \% \mathrm{CI}$ using a matched-pairs analysis followed by step-wise logistic regression on all the variables. Multivariate analysis was used to control for confounding factors.

Consumption of soy formula was found to be associated with premature thelarche in the univariate analysis $(\mathrm{OR}=2.2,95 \% \mathrm{CI}=1.0-5.2, P=0.05$ based on 22 exposed cases and 10 exposed controls). A maternal history of ovarian cysts was also significantly associated with premature thelarche on univariate analysis $(\mathrm{OR}=3.8,95 \% \mathrm{CI}=1.5-11.5, P=0.002)$. Multivariate analysis showed no significant associations overall. When the analysis was restricted to girls with onset of thelarche before age 2 years, consumption of soy formula remained significantly associated in multivariate analysis (Table 26). The study authors noted that in $>50 \%$ of the subjects, there was no exposure to any of the risk factors for which statistical associations were found. They suggested that premature thelarche resulted from a variety of environmental and familial factors.

Strengths/Weaknesses: The case-control study design is a strength. Other strengths are the use of age-matched controls, the appropriate matched and multivariate analyses, and the reasonable sample size. Weaknesses include the broad age range of the girls with varying lengths of delay in collecting retrospective history and the use of friend controls in some cases but not in others. There could have been recall bias; for example, factors associated with thelarche before age 2 (in multivariate analyses) should have controlled for the age at which the maternal interview was conducted. Other potential confounders were not addressed, and the reasons why soy formula was used initially could have been a confounding association.

Utility (Adequacy) for CERHR Evaluation Process: This study is useful for the evaluation process.

Strom et al. (2001), supported by Ross Products, Nestlé, and Mead Johnson, compared endocrine and reproductive outcomes in adults who had been fed soy or cow-milk formula during infancy. Subjects were identified from a group of 952 individuals ( 248 fed soy formula and 563 fed cow-milk formula) who participated in controlled non-randomized infant feeding studies conducted at the University of Iowa between 1965-1978. In the feeding study, infants were enrolled before 9 days of age and fed either a cow-milk or soy formula through 16 weeks of age. The current study was conducted from March through August of 1999. Subjects included 248 individuals ( 120 male, 128 female) fed soy formula and 563 individuals (295 males, 268 females) fed cow-milk formula. Most subjects were Caucasian and were between 20-34 years of age. Information was obtained by structured and standardized telephone interviews with study participants. Men and women were asked about adult heights and weights, sexual maturation, and education level. Women were also questioned about menstrual cycles (cycle length, menstrual duration, flow, and pain) and reproductive outcomes (number of pregnancies, deliveries, and complications). Men were also asked about pregnancy outcomes in partners. Additional questions included congenital malformations in offspring, hormonal disorders, and homosexual orientation in both sexes, and testicular cancer in men. These outcomes were considered secondary and were expected to occur too infrequently to provide definitive data. Ordinal variables were analyzed by $\chi^{2}$ or Fisher exact tests, and unadjusted and adjusted relative risks were calculated. Continuous variables were analyzed by $t$-test or Wilcoxon rank sum test followed by linear regression. Confounding factors considered in the analysis included birth weight, age, usual body mass, parental weight and height, hormone disorders, smoking, alcohol intake, soy-food consumption, vegetarian diet, herbal supplement 
use, recreational drug use, physical activity, sexually transmitted diseases, and use of hormonal birth-control methods.

No significant differences were detected in adult height and weight, body mass index, or sexual maturation in men and women. Duration of menstrual bleeding was slightly longer in women from the soy-formula group (adjusted mean difference $=0.37$ days, $95 \% \mathrm{CI}=0.06-0.68$ ), but bleeding was not heavier. Borderline significance was obtained for the number of subjects experiencing discomfort during menstruation in the soy-formula group (unadjusted $\mathrm{RR}=1.77$, 95\% CI $=1.04-3.00$ for extreme vs. no or mild discomfort; adjusted RR was not calculable). No significant differences were identified for pregnancy outcomes in women. [No reproductive parameters were reported for male subjects with the exception of sexual maturation. Although men were questioned about pregnancy outcomes in partners, the results were not reported.] No significant effects were observed for secondary outcomes (i.e., cancer, reproductive organ disorders, hormonal disorders, sexual orientation, or defects in offspring [data were not shown]).With adjustment for multiple comparisons, the differences that had been identified were no longer statistically significant ( $>30$ different endpoints were evaluated).

The study authors concluded that "Exposure to soy formula does not appear to lead to different general health or reproductive outcomes than exposure to cow-milk formula. Although the few positive findings should be explored in future studies, our findings are reassuring about the safety of infant soy formula."

In a letter to the editor, Goldman et al. (2001) discussed several perceived limitations of the Strom et al. (2001) study:

- Failure to mention in study abstract a higher use of asthma and allergymedications in subjects fed soy-based formula ( $P=0.08$ in males and $P=0.047$ in females),

- Disregarding the slightly increased duration of menstrual cycle and greater menstrual discomfort findings in soy formula-fed women, when those findings could indicate endometriosis or uterine fibroids, theoretical outcomes of early-life estrogen exposure,

- Inadequate assessment of female fertility by not considering time to pregnancy and use of fertility technologies and only asking subjects about subjective outcomes,

- Lack of reporting of male fertility outcomes or semen quality,

- Lack of assessment of cancer risk, and

- Lack of consideration of exposure to estrogenic compounds in various soy-based formulas.

In response to this letter, Strom et al. provided incidences for thyroid disease (a possible autoimmune disease), endometriosis, uterine fibroids, low sperm count, and cancer in subjects fed soy versus cow-milk formula (Table 27). They noted the low number of subjects affected.

Strengths/Weaknesses: Strengths of the study include the large sample, the prospective collection of feeding information, and the appropriate statistical analyses. Feeding assignment was, however, not randomized, soy-formula exposure was short-term (0-16 weeks of age), and information on subsequent feeding other than current vegetarian diet was not collected. Sample sizes for some analyses (cancer, reproductive organ disorders, hormonal disorders, libido dysfunction, sexual orientation, and birth defects in the offspring) were too small to be definitive. It is possible that there was a bias in subjects who were able to be located compared to those not located 30 years later, and there may have been inaccurate recall for some measures 
collected at follow-up. The measures of infertility were weak, and the clinical relevance of the subjective measure of menstrual bleeding is questionable.

Utility (Adequacy) for CERHR Evaluation Process: This study is useful for the evaluation process.

Giampietro et al. (2004), funding not indicated, conducted a retrospective study to determine the hormonal and metabolic effects of long-term feeding of soy formula in children. The study population consisted of 48 children age 7-96 months who had been fed soy formula exclusively for at least 6 months. Of these children, 30 were given soy formula for documented cow-milk allergy and 18 for family history of allergy. The 48 children were divided into three age groups, 7-24 months $(\mathrm{n}=20), 25-72$ months $(\mathrm{n}=20)$, and 73-96 months $(\mathrm{n}=8)$. An additional 18 healthy children aged 12-96 months who had not received soy formula during the first months of life were enrolled as a control group. The children were evaluated for height, weight, presence of breast budding, testicular size, presence of pubic/axillary hair, signs of precocious puberty in girls, and the appearance of gynecomastia in boys. Also studied were radiologic and biochemical markers of increased bone metabolism, including bone age, urinary deoxypyridoline, calcium, creatinine, and phosphate and serum levels of bone alkaline phosphatase, osteocalcin, 17 $\beta$-estradiol, and parathyroid hormone. Univariate analyses were carried out on the data, using the Fisher exact test and $\chi^{2}$ test for discrete variables and the Mann-Whitney test for continuous nonparametric variables.

Height and weight were in the normal range for all children, and no differences were detected between soy formula-fed and control groups. No signs of precocious puberty in girls or of gynecomastia in boys were found. All $17 \beta$-estradiol concentrations were below the method detection limit of $20 \mathrm{pg} / \mathrm{mL}$. No significant differences were detected in serum or urinary measurements, except that soy formula-fed infants had significantly lower urinary calcium and significant higher urinary phosphate in children 7-24 months old compared to control children.

The authors concluded that long-term feeding of soy formula in infants did not produce estrogen-like hormonal effects. They suggested that their findings confirm the theory that phytoestrogens have a low affinity for ERs and therefore produce only weak biologic effects. The authors suggested that the isolated differences in urinary calcium and phosphate should be confirmed in larger studies before conclusions are drawn.

Strengths/Weaknesses: It is a strength that exclusive feeding of soy formula was apparently at least 6 months in duration. Use of a comparison group of unexposed infants in the first months of life and multiple measures of possible hormonal effects are other strengths.

Weaknesses include the retrospective enrollment, in some cases many years after infancy, and the lack of clarity on how feeding patterns in early life were validated. The feeding practices in the comparison group were not well described. There were no multivariate analyses or controls for potential confounders. Sample sizes in each age group were small, raising the question of the power to identify group differences.

Utility (Adequacy) for CERHR Evaluation Process: This study is of limited utility for the evaluation process.

3.1.2.6 Cholesterol: Cruz et al. (1994), supported by NIH, the Perinatal Research Institute, USDA, and Ross Laboratories, conducted a prospective, partially randomized, controlled study to evaluate the effects of cholesterol and phytoestrogen intake on cholesterol-synthesis rates in infants. Thirty-nine normal term Caucasian male infants were recruited from area hospitals. Of 33 infants who completed the study, 12 received human milk (cholesterol $2.59-3.88 \mathrm{mM}$ ) for the first 4 months of life, with occasional supplemental cow-milk formula (Similac ${ }^{\circledR}$ with 
Iron; cholesterol $0.28-0.85 \mathrm{mM}$ ). Twenty of 26 remaining infants were randomized to receive either cow-milk formula or soy formula (Isomil ${ }^{\circledR}$ with Iron; cholesterol $0 \mathrm{mM}$ ). The remaining six infants were non-randomly assigned to receive a modified-soy formula with the same cholesterol concentration $(0.28 \mathrm{mM})$ as the cow-milk formula. Infants received their assigned formulas beginning at age 3-7 days and continuing until age 4 months, with no additional nutrition received other than multivitamins. At 4 months, cholesterol-synthesis rates and urinary isoflavone excretion were determined, with measurements taken over a 4-day period. Serum cholesterol and lipid profiles were determined using validated enzymatic techniques. Serum LDL cholesterol concentrations were calculated from serum total cholesterol. Cholesterol fractional synthesis rate [the percent cholesterol synthesized per day] was determined from the rate of incorporation of deuterium into red blood cell-membrane cholesterol. Urinary isoflavones were determined by GC-MS. Data were analyzed using 1-way ANOVA with post-hoc Tukey-Kramer test. Correlations between cholesterol intake and cholesterol fractional synthesis rates were determined using a nonparametric test for ordered alternatives.

Data for serum lipid profiles and fractional synthesis-rate determinations are shown in Table 28. The highest values for serum total and LDL cholesterol were found in breast-fed infants. These infants had the lowest fraction of synthesized cholesterol. There was a significant negative association between cholesterol intake and the fractional cholesterol-synthesis rate $(P<0.0001)$. Isoflavone excretion was associated with soy-formula consumption. Multiple regression analysis controlling for group effect found total urinary isoflavone excretion to be negatively associated with fractional cholesterol-synthesis rate but not with serum cholesterol concentration.

The study authors concluded that infants responded to different dietary cholesterol intakes through altered cholesterol-synthesis rates and that infant diet can affect serum lipid profiles, cholesterol-synthesis rates, and urinary isoflavone excretion.

Strengths/Weaknesses: After selection for breast feeding, random assignment to primary soy or cow-milk formula groups and documentation of no additional nutrition to 4 months are strengths. The inclusion of only males is a weakness. Selection bias was possible for breast feeding mothers. The modified soy-formula group was not included in randomization, and the multivariate analysis results were not described very clearly.

Utility (Adequacy) for CERHR Evaluation Process: This study is useful for the evaluation process.

3.1.2.7 Diabetes mellitus: Fort et al. (1986), funding not indicated, examined feeding histories of 95 diabetic children and controls to study the effect of breast feeding on the development of insulin-dependent diabetes mellitus. The feeding histories of the diabetic children were compared to those of a control group consisting of 194 non-diabetic siblings and an additional 95 non-diabetic friends living in the same geographic area. Data were analyzed by a sign test and $\chi^{2}$ test. Eleven of 95 diabetic children were excluded from statistical analysis because they changed addresses or could not be matched. No significant differences were detected in the incidence or duration of breast feeding among the groups, or in the introduction of solid foods. Almost twice as many diabetic children had been fed soy formulas compared to controls.

[Data on soy formulas were not shown. The authors noted that most children on formula received cow-milk formula and that the group differences in soy-formula intake were not statistically significant.] The authors postulated that diabetic infants might have a higher incidence of gastrointestinal alterations giving rise to a switch to soy formula. 
Strengths/Weaknesses: The case control design is a strength as is the use of sibling controls and unrelated controls matched by geographic area. Weaknesses include the retrospective collection of infant feeding information an average of 14 years later. The soy-formula exposure was not specified and could have been with breast feeding or not. Specific numbers were not presented for soy formula; an association was identified but there were no statistically significant differences, making conclusions difficult.

Utility (Adequacy) for CERHR Evaluation Process: This study is somewhat useful for the evaluation process.

3.1.2.8 Cognitive function: Malloy and Berendes (1998), funding not indicated, studied the relationship between breast feeding during infancy and cognitive performance in 9- and 10year-old children. Cognitive testing and health results were obtained for children in a 19781979 birth cohort. All 518 infants in the study had received soy formula. Of these infants, 342 had been breast-fed and 176 had never been breast-fed. Dietary information was obtained through questionnaires completed by the parents. The Wechsler Intelligence Scale for Children-Revised (WISCR) verbal, performance, and full-scale intelligence quotients (IQ) were administered to children in their homes. Data were analyzed using $\chi^{2}$ and Student $t$-test. A linear regression model was used to adjust least-square means for potential confounding variables. Differences were considered significant at $P \leq 0.05$.

The researchers initially found higher WISC-R verbal and full-scale mean scores among the breast-fed children. After adjustment for maternal education, paternal education, and annual family income, the differences were no longer significant. The authors concluded that in this relatively socio-economically advantaged population, additional advantages of breast feeding might be more difficult to demonstrate.

Strengths/Weaknesses: The use of a birth cohort. standardized testing in school-age children within a narrow age range, and the large sample size are strengths. Weaknesses include the retrospective collection of infant feeding information only by questionnaire 9-10 years later and the comparison only between children who were breast-fed for any length of time and children who were exclusively soy formula-fed. If any real differences existed, they could have been diluted by soy exposure in the breast-fed group.

Utility (Adequacy) for CERHR Evaluation Process: This study is somewhat useful in the evaluation process.

3.1.3 Exposure during adolescence-Shu et al. (2001), supported by the National Cancer Institute, used a case-control design to evaluate the association between breast cancer in adulthood and soy-food intake during adolescence. Women between the ages of 25-64 years with newly diagnosed breast cancer were recruited. The women, who were permanent residents of Shanghai, were selected from a cancer registry. Controls were selected from a registry of residents of Shanghai and were matched to cases on age (within 5-year intervals). Of the women identified, $91.1 \%$ of cases $(n=1459)$ and $90.3 \%$ of controls $(n=1556)$ agreed to participate. The height and weight of each participant was recorded, and participants were interviewed regarding demographic factors, menstrual history, reproductive history, hormone use, past illnesses, physical activity, tobacco and ethanol use, weight, and family history of cancer. A food frequency questionnaire was used to determine adult and adolescent (age $=13-15$ years) dietary intake. In a subgroup of 569 cases and 659 controls who were $<45$ years old at interview, attempts were made to contact the participants' mothers for assessment of their daughters' adolescent dietary intake as well as pregnancy and lactational exposures. Interviews were completed for $79.6 \%$ of case mothers and $81.8 \%$ of control mothers. Soy intake was estimated by combining the soy-protein content of specific foods and was divided into quintiles. 
Unconditional logistic regression was used to obtain OR and 95\% CI with control for potential confounders (age, education, family history of breast cancer, history of fibroadenoma, body mass index, waist:hip ratio, and exercise level.) [The data table also indicates adjustments for rice and wheat intake, pregnancy experience, age at menarche, and age at menopause.]

Results are summarized in Table 29. There was a significant inverse association between adolescent soy intake and risk of breast cancer. This significant inverse association was found when total soy intake was considered and when individual categories of soy food (tofu, soy milk, other soy food) were considered. There was an inverse association between rice/wheat and milk intake and risk of breast cancer. Controlling for soy intake did not eliminate the rice/ wheat or milk associations. Intake of fresh legumes and other categories of foods was not associated with breast cancer risk. The inverse association between adolescent soy intake and breast cancer persisted when adjusted for adult soy intake, suggesting to the authors that adolescent soy intake was not just a proxy for lifetime or adult soy intake. The authors recognized the potential inaccuracy of diet recall, but they believed that any recall errors would be non-differential because the hypothesized protective effect of soy against breast cancer was not known in China during the study period.

Strengths/Weaknesses: The case-control design using age-matched controls is a strength. The use of an Asian population likely to have high exposures, the large sample, and the high participation rate are additional strengths. The dose-response relation shown in the recall of the women and the women's mothers reinforces the identification of the association.

Weaknesses include the lumping of pre- and post-menopausal breast cancer cases, the potential for recall bias, and the possibility that the food frequency questionnaire may not have represented true intake. In addition, for the purposes of this evaluation, the evaluation of soy food rather than soy formula is a weakness.

Utility (Adequacy) for CERHR Evaluation Process: This study is useful in the evaluation process.

Wu et al. (2002), in a study supported by the California Breast Cancer Research Program, examined the relationship between soy intake in adolescence and breast cancer in adulthood. Volunteers selected for the case-control study were of Asian-American descent, specifically Chinese, Japanese, or Filipino, and lived in Los Angeles county. From 1995, 501 cancer cases were selected from the Los Angeles County Cancer Surveillance program. The patients were 25-74 years old at the time of breast cancer diagnosis. Controls $(n=594)$ were selected from neighborhoods where cancer patients lived at the time of diagnosis and were matched according to ethnicity and age. Volunteers were interviewed about demographic characteristics, migration history, menstrual and reproductive history, body size, physical activity, and diet history. Soy intake in adulthood was estimated by asking volunteers about the frequency and volume of various soy foods ingested (e.g., miso, tofu, vegetarian meats, soybeans, and natto). Crude estimates of soy product intake in adolescence (12-18 years of age) were based on reported frequency of tofu ingestion. Isoflavone intake in adulthood was estimated using data from the Hawaii Food Composition Database. Odds ratios were calculated by conditional logistic regression analysis with matching for age and specific Asian ethnicity. Odds ratios were adjusted for education, birthplace, age at menarche, parity, current body mass, menopausal status, and use of menopausal hormones. A second set of adjustments was made for intake of dark leafy vegetables during adolescence (considered an indication of a traditional Asian diet), smoking history, alcohol intake, physical activity, and family history of breast cancer. Odds ratios were calculated for quartiles of soy intake during adolescence ( $<1$ times/ month, 1-3 times/month, 1-3 times/week, $\geq 4$ times/week) and isoflavone intake during adulthood $(\leq 1.79,>1.79-6.24,>6.24-12.68$, or $>12.68 \mathrm{mg} / 1000 \mathrm{kcal})$. 
Soy product intake was highest in Chinese (26.8mg/day), intermediate in Japanese (18.4 mg/ day), and lowest in Filipino ( $9.3 \mathrm{mg} /$ day) volunteers. Statistically significant ORs (i.e., one not included in 95\% CI) are summarized in Table 30. There were some significant and inverse associations between soy intake in adolescence or adulthood and risk of breast cancer in adulthood. When exposures during both adolescence and adulthood were considered, only high exposures during both adolescence and adulthood suggested a significant protective effect against breast cancer. There was no significant effect of high-soy exposure in adolescence and low-soy exposure in adulthood (OR, $95 \% \mathrm{CI}=0.77,0.51-1.10$ ). There was also no significant association for low-soy exposure in childhood and high-soy exposure in adulthood and decreased risk of breast cancer ( $\mathrm{OR}, 95 \% \mathrm{CI}=0.93,0.58-1.48)$. The study authors concluded that the study showed an association between high intake of soy in childhood and reduced breast cancer risk in Asian-Americans. The study authors also noted that breast cancer risk may be reduced further by soy intake in adulthood.

Strengths/Weaknesses: This well constructed casecontrol study had a large sample size with appropriate matching of controls using matched analytical methods. Multivariate analyses controlled for important potential confounders. Weaknesses include the very wide age range of breast cancer cases and controls, so time-to-recall of adolescent tofu intake apparently ranged from about 6 years to about 55 years. There is also potential for recall bias in a case-control study design with normal controls. Measure of adolescent intake was based solely on recall of tofu intake, which could have misrepresented total intake.

Utility (Adequacy) for CERHR Evaluation Process: This study is useful for the evaluation process.

Maskarinec et al. (2004b), supported by the National Cancer Institute, evaluated recall of early life soy food intake in relation to mammographic densities. Subjects included 220 women [the subjects of a study (Maskarinec et al., 2004a) discussed in Section 4.1.1] with a mean age of $\sim 43$ years who were given a one-page questionnaire on soy product exposure during infancy, childhood (age $=1-9$ years), adolescence (10-19 years), early adulthood (20-29 years), and late adulthood ( $\geq 30$ years). Based on the amount of each soy food indicated, number of servings was calculated for each life stage and soy intake was dichotomized as low or high based on the relationship of each woman's intake to the median number of servings. [In the presentation of data, soy product intake was dichotomized as none or $\geq 1$ serving/year for a combined 0-19 year age group.] The baseline percentage of dense breast tissue was higher for women who consumed greater than one soy product serving/year during ages 0-19 years compared to women who had consumed no soy products during this time (49.8\% [n $=108]$ compared to $44.0 \%$ [ $=110] ; P=0.08$, adjusted for body-mass index, Asian ethnicity, number of children, and age at first birth). In the 2-year intervention, women were randomized to maintain their baseline diet or to eat two servings of soy foods/day. Breast density decreased over the 2 years in both groups, but the rate of decrease was lower in women who reported any soy food intake in early life than women who reported no soy food intake in early life. By contrast, soy food intake after age 20 was associated with a more rapid decrease in breast density. The authors found the difference between effects of early life and young adulthood soy food exposure to be puzzling and suggested that unmeasured confounding factors may have been present.

Strengths/Weaknesses: This study presents recalled early soy intake from a study (Maskarinec et al., 2004a) that is described in Section 4.1.1 and is also discussed there. This study is one of only a few examining the association between soy food exposure and breast endpoints. The methods used to measure hormones were good and there was good compliance with the study protocol. A weakness is that there was no control for the scheduling of mammograms. Breast density is not breast cancer, and the authors' assumptions about soy food intake and breast cancer risk are not supported by data in this study. 
Utility (Adequacy) for CERHR Evaluation Process: This study is useful in the evaluation process.

\subsection{Experimental Animal Data}

3.2.1 Development of the reproductive system-The effects of developmental soy exposure on reproductive endpoints were examined in numerous rat studies, one marmoset study, one rhesus monkey study, and one mouse study. Studies in rats are presented first, in order of female effects followed by male effects. Within each sex, studies with exposures commencing during the prenatal period are presented before studies with exposures beginning in the postnatal period. Studies in mice and monkeys are presented at the end of this section.

Hughes et al. (2004), supported by EPA, examined the offspring of dams treated with soy milk during the lactation period. A similar study was conducted in rats dosed with genistein and is discussed in the CERHR Expert Panel Report on Genistein (Rozman et al., in press). This study was conducted in Long-Evans hooded rats that were fed a phytoestrogen-free AIN-93G diet in which the soy oil was replaced with corn oil. Dams were randomly assigned to groups given $2 \%$-fat rice milk (controls, $\mathrm{n}=2$ ) or $2 \%$-fat soy milk $(\mathrm{n}=3$ ) instead of water from PND 1 (day of delivery) to PND 21. The study authors estimated that intake of isoflavones through soy formula was $3.5-8.5 \mathrm{mg} /$ day or $0-30 \mathrm{mg} / \mathrm{kg}$ bw/day. Pups had some direct exposure to the soy or rice milk once they were old enough to drink from the dam's bottle. Milks were replaced with water from PND 21 to the end of the study. Litter size was evaluated at birth, and pups were sexed, weighed, and evaluated for anogenital distance. Body weights and anogenital distance were again measured at weaning. Pups were monitored for onset of puberty. Estrous cycles were monitored for 3 weeks in females. On PND 60, offspring were killed and male reproductive organs were weighed. A total of 14 male and 22 female pups exposed to soy milk and 8 male and 18 female pups exposed to rice milk were evaluated. Uteri from six offspring/ group were fixed in $4 \%$ paraformaldehyde for histomorphometry examination and immunohistochemical analyses for proliferating cell nuclear antigen (PCNA), ER $\alpha$, and progesterone receptor. Statistical analyses included ANOVA and Kruskal-Wallis test. The individual pups rather than the litter were considered the statistical unit. The pup-based analysis was said to have been used because intrauterine position of pups, which was not considered, was said to have a greater impact on variances of outcomes than differences between dams.

No effects of soy milk were detected on anogenital distance, age of preputial separation, age of vaginal opening, or estrous cycle length. Body weight was significantly higher and anogenital distance adjusted for body weight was significantly lower in female offspring of the soy group at weaning. Soy milk had no effect on relative (to body weight) weights of testis, seminal vesicles, or prostate in adult offspring. Relative epididymis weight was significantly reduced [by 14\%] in the soy group compared to the rice-milk control. [Absolute organ weights were not reported.] The only significant effect noted in the uterus was a [12\%] increase in progesterone receptor expression in glandular epithelial cells in females of the soymilk group. Soy milk had no effect on uterine gland numbers, luminal epithelial cell height, uterine proliferation, ER $\alpha$ expression in luminal and glandular epithelial cells, or progesterone receptor expression in luminal epithelial cells. As discussed in the CERHR Expert Panel Report on Genistein (Rozman et al., in press), gestational and lactational exposure to genistein also increased expression of the progesterone receptor in uterine glandular epithelial cells. The study authors concluded that exposure of developing rats to isoflavones approximating human exposure levels induced an effect in an estrogen-responsive uterine marker long after cessation of exposure. Concerns were expressed because the progesterone receptor is involved in several reproductive processes. 
Strengths/Weakness: Strengths of this study were use of a phytoestrogen-free chow, exposure during an appropriate period (lactation), exposure of rats to soy milk, and estimation of daily isoflavone intake. Weaknesses were the small numbers of litters used/group (2-3) and that the litter was not considered the experimental unit.

Utility (Adequacy) for CERHR Evaluative Process: This study is of limited utility due to the small numbers of animals used.

Ashby et al. (2000b), examined the effects of Infasoy®, an infant soy formula, and animal feeds on uterotropic responses and initiation of puberty in rats. The uterotropic effects are summarized in Section 2.2.4.2. Two separate experiments were conducted. In the first experiment, a control group of 34 rats from five litters was fed RM1 [Rat and Mouse No. 1, a soy-based diet] and a comparison group of 29 rats from six litters was fed AIN-76 (a phytoestrogen-free diet). In the second experiment, a control group of 49 rats from nine litters was fed the RM1 diet and a comparison group of 61 rats from 10 litters was given Infasoy ${ }^{\circledR}$ in drinking water. In both experiments, rats were exposed to each diet or treatment on PND 21-55. Body weights and age of vaginal opening and first estrus were monitored. Data were analyzed by ANOVA and ANCOVA, with the litter as the unit of analysis. Results are summarized in Table 31. Vaginal opening and first estrus occurred at a younger age in rats fed the AIN-76A compared to the RM1 diet. Body weights of rats fed the AIN-76-A diet were higher on PND 35 but lower on day of vaginal opening. Age of vaginal opening and first estrus were accelerated in the rats given Infasoy ${ }^{\circledR}$ compared to control rats fed the RM1 diets. Body weights of rats given Infasoy ${ }^{\circledR}$ were higher than control rats on PND 46 but were lower than control rats on the day of vaginal opening and first estrus. As explained in greater detail in Section 2.2.4.2, uterotropic responses were attenuated by the ER $\alpha$ and $\operatorname{ER} \beta$ antagonist Faslodex, the aromatase inhibitor anastrozole, and the GnRH antagonist antarelix. The study authors noted that sexual development in rodents can be accelerated by exogenous synthetic or dietary estrogens interacting with tissue ERs or through a centrally mediated increase in endogenous estrogens. The study authors concluded that dietary components other than contaminant synthetic estrogens or phytoestrogens can accelerate sexual development in rodents and may therefore be a factor in precocious human development.

Strengths/Weakness: Strengths of the study were use of soy milk, exposures only postnatally (PND 21-55), adequate sample size ( $\mathrm{n}=9-10)$, and use of the litter as the experimental unit. Weaknesses include the lack of information on phytoestrogen content of the chow, the failure to monitor liquid consumption, resulting in unknown exposure to soy, the use of a soy-based chow (RM1) with experimental exposure to Infasoy, resulting in two sources of soy exposure, the use of only one soy formula dose level, and the lack of clarity on how animals were chosen for analysis (presumably all females from all litters evaluated).

Utility (Adequacy) for CERHR Evaluative Process: This study is of low utility based on the single dose level of soy formula and the complication of exposure to soy in both the diet and the liquid.

Odum et al. (2001), supported by the Alkyl Phenol Ethoxylate Research Council, the Japanese Chemical Industries Association, and the Food Standards Agency [a U.K. government agency], investigated the effects of different rodent diets on sexual development in the rat. Animals were Wistar-derived Alpk:APfSD rats. The diets included Rat and Mouse No. 3 (RM3), a standard diet in the U.K. for pregnancy and lactation, RM1, a standard diet for maintenance of rodents, Teklad Global 2016 (from Harlan Teklad), a natural diet made without soy or alfalfa, AIN-76A (from Harlan Teklad), a diet with sucrose, casein, and maize instead of soy and alfalfa, and Purina 5001 (from Purina Mills), a common U.S. diet based on soy and alfalfa. Features of these diets are shown in Table 32. Diet combinations included a specified 
diet during pregnancy and lactation followed by the same or a different diet for weaned offspring. The pregnancy/weaning treatment diets were RM3/RM1, AIN-76A/AIN-76A, RM3/AIN-76A, Global 2016/Global 2016, and 5001/5001. Pregnant females were begun on their assigned diets on the day sperm were detected in the vaginal smear (GD 0). There were 12 females assigned to each of the five diet combinations. Genistein and daidzein content were measured in each diet by GCMS. Females were maintained on the assigned diets through pregnancy and lactation. Offspring were maintained on their assigned diets until PND 68. From PND 70, offspring were all given the RM1 diet [the diet between PND 68 and 70 was not specified]. All females from six litters per treatment group were killed on PND 26, and sex organs were weighed. The remaining females were housed together by litter. Males were culled at weaning to four per litter (housed together). Developmental landmarks were monitored, including eye opening, testis descent, vaginal opening, and preputial separation. After vaginal opening, daily vaginal smears were taken until first estrus was identified. Daily vaginal smears were also taken on PND 52-69 to evaluate time spent in estrus. On PND 68, males from six litters per treatment group were killed and liver, kidney, and sex organs were weighed. On PND 68, the remaining females were culled to four per litter (housed together). The remaining males were killed on PND 128, and the remaining females were killed in estrus on PND 140144 (after being on the RM1 diet since PND 70). Liver, kidneys, and sex organs were weighed. The RM3/RM1 diet combination was considered the control condition because this combination (for pregnancy/weaning) was used in most of the authors' previous studies. The $5001 / 5001$ diet combination was also evaluated as a control diet, because it is used in many regulatory studies in the U.S. [Analysis using the 5001 diet as a control was not shown in the study.] Statistical analysis was carried out by ANOVA or ANCOVA (taking weaning weight as a covariate) with post-hoc Student $t$-test. The litter was the statistical unit. The proportion of animals recorded with a developmental landmark each day was evaluated by Fisher exact test.

Pregnancy outcome and offspring weight results are summarized in Table 33. [Post-weaning food consumption and weight varied over the course of the experiment. The data graphs are difficult to interpret, but it seems that AIN-76A and Global 2016 may be suboptimal feeds for the post-weaning period in terms of body weight gain, feed consumption, or both. The authors note that the high-energy density of these diets may have influenced feed consumption. The authors imply that during the "recovery phase" after PND 70, when all animals were given RM1, feed consumption and weight tended to become similar among groups, although it did not reach parity in all groups.] There were no effects of diet on eye opening. The effects of the diets on sex-related developmental landmarks are summarized in Table 34. Post-weaning exposure to AIN-76A appeared to consistently advance male and female pubertal landmarks compared to RM1, whereas the other two diets had less consistent effects. Effects on body weight and reproductive organ weight are summarized in Table 34. Absolute body weight and absolute organ weights were not evaluated for statistical differences; Table 34 shows the effects of diet only with regard to body weight corrected for weaning body weight and with regard to relative organ weights. The authors noted that AIN-76A was associated with body weight gain that persisted during the recovery period, i.e., during the weeks after PND 70 when all animals were on the RM1 diet.

In a second experiment, weanling female rats were obtained at 20-21 days of age. Animals were weaned on RM3 and then switched to RM1, AIN-76A, Global 2016, or 5001 ( $\mathrm{n}=24$ / group) for 4 days. Half of the animals in each group received, respectively, 0 or $300 \mu \mathrm{g} / \mathrm{kg} \mathrm{bw} /$ day subcutaneously (s.c.) of the GnRH antagonist antarelix for 3 days starting on PND 20 or 21. Animals were killed $24 \mathrm{hr}$ after the last dose of antarelix. Body and uterine weights were compared with ANCOVA, considering initial body weight (for final body weight) and final body weight (for uterine weight) as covariates. The individual female was the statistical unit. Results are summarized in Table 35 for final body weight (adjusted for initial body weight) 
and relative uterine weight. Absolute body and uterine weights were not analyzed statistically. Antarelix increased feed consumption by $24-40 \%$ [data not shown].

The authors concluded that diet could affect sexual development, but they observed that the effects on sexual development were not consistent with presumed estrogenicity of diets like 5001 that are high in phytoestrogens. They noted, for example, that the greatest effect on sexual development appeared with post-weaning exposure to AIN-76A, a soy- and alfalfa-free diet with negligible phytoestrogen content. They further noted that in the uterotropic assay, AIN-76A and 5001 produced an increase in relative uterine weight that was prevented with a GnRH antagonist, suggesting that the effects of diet involved alterations in hypothalamic function rather than simple estrogen exposure.

Strengths/Weakness: Strengths of the study were analysis of diet for genistein and daidzein content and analysis of appropriate endpoints. Weaknesses were differences in dietary protein and metabolizable energy content. Exposure through chow is a strength in its relevance to human exposure and a weakness in permitting only imprecise estimates of dose levels.

Utility (Adequacy) for CERHR Evaluative Process: This study is of low-to-moderate utility based on the fairly large number of animals examined and the appropriateness of endpoints.

Lund et al. (2001a), supported by the National Science Foundation and Brigham Young University, conducted a series of experiments to examine reproductive and developmental effects in Long-Evans rats given phytoestrogen-containing feed. The $\mathrm{F}_{1}$ rats used in these studies were born to five $\mathrm{F}_{0}$ dams/group that were fed throughout the gestation and lactation periods with a soy-based diet containing phytoestrogens $600 \mu \mathrm{g} / \mathrm{g}$ diet (phyto-600) or a phytoestrogen-free fish meal-based diet. The offspring were weaned on PND 30 to the same diets as their dams. Diets were similar in protein, fat, amino acid, mineral, and vitamin content. Assessment of estrous cycles in $\mathrm{F}_{0}$ rats is discussed in Section 4.2. Data were analyzed by ANOVA followed by Bonferroni post-hoc comparison.

In the first part of the experiment, female offspring $(n=16 /$ group $)$ were monitored for day of vaginal opening and estrous cyclicity from 60-73 days of age. Body weights were measured in male and female offspring at 50 days of age $(n=20 /$ sex/group). In the phyto- 600 compared to phytoestrogen-free group, day of vaginal opening was significantly delayed (mean $\pm \mathrm{SEM}=$ $39.3 \pm 0.5$ days compared to $35.0 \pm 0.3$ days in the control). Diet had no effect on length or regularity of estrous cycles [data were not shown]. Body weights at 50 days of age were significantly lower in males and females fed the phyto-600 compared to the phyto-estrogenfree diet [by $\sim 10 \%$ in females and $\sim 6 \%$ in males].

In the second part of the study, five to seven rats/sex/group given the phyto-600 diet continued to receive that diet, and six rats/sex/group were switched to the phytoestrogen-free diet at 80 days of age. Rats remained on each diet for 40 more days (until 120 days of age), at which time they were killed. Blood from two rats/group was pooled for measurement of serum phytoestrogen levels by GC-MS and measurement of $17 \beta$-estradiol and testosterone levels by radioimmunoassay (RIA). Body and prostate weights and volume of the sexually dimorphic nucleus of the pre-optic area (SDN-POA) were determined ( $n=5-7 /$ sex/group). Body weights of male and female rats switched to the phytoestrogen-free diet were significantly higher than body weights of rats that remained on the phyto-600 diet [7\% higher in males and $13 \%$

higher in females]. Males switched to the phytoestrogen-free diet also had higher absolute $[\sim 21 \%]$ and relative [ $\sim \mathbf{1 5 \%}$ ] prostate weights. Diet had no effect on serum testosterone or $17 \beta$-estradiol level in males. Males that remained on the phyto-600 diet had a significantly higher SDN-POA volume than males switched to the phytoestrogen-free diet. An increase in SDN-POA volume in females switched to the phytoestrogen-free diet compared to females 
remaining on the phyto-600 diet approached statistical significance. Total brain weight did not differ between dietary groups.

The study authors concluded that, "...phytoestrogens have considerable effects on hormonally sensitive somatic, reproductive organ, and neuroendocrine parameters...."

Strengths/Weakness: Strengths of this study were detailed descriptions of diets, measurement of serum phytoestrogens, and examination of appropriate endpoints. Weaknesses were comparison of only one phytoestrogen-containing chow to phytoestrogen-free chow, and the small number of animals used $(n=5)$. Exposure through chow is a strength in its relevance to human exposure and a weakness in permitting only imprecise estimates of dose levels. In addition, the litter was not considered the experimental unit, and reproductive capability of animals was not determined.

Utility (Adequacy) for CERHR Evaluative Process: This study is of limited utility. The effect of switching the diet at 80 days of age is not relevant to developmental events.

Masutomi et al. (2004), in a study supported in part by grants from the Japanese Ministry of Health, Labor, and Welfare, examined the effects of maternal diet in rats perinatally exposed to ethinyl estradiol. Pregnant Sprague-Dawley rats $(n=15$ /group) were divided into groups fed CRF-1, a regular rodent diet containing soy proteins, or a soy-free diet. The CRF-1 diet contained genistein at $10.2 \mathrm{mg} / 100 \mathrm{~g}$ diet and daidzin at $8.7 \mathrm{mg} / 100 \mathrm{~g}$ diet. Soy-free diet was prepared according to the NIH-07 formulation, except that soy meal and oil were replaced with ground corn, wheat, and corn oil. [It also seems that soy-free diets may have used fish or gluten meal as the source of protein.] On GD 15 (day of vaginal plug = GD 0), 7 dams from each dietary group were given $0.5 \mathrm{ppm}$ ethinyl estradiol through diet. Dosing was continued through PND 10 (day of delivery = PND 1). Pup anogenital distance was measured on PND 2. Five offspring/sex/group were necropsied on PND 21 for measurement of organ weight. Eight offspring/sex/group were weaned to the CRF-1 diet and evaluated for onset of puberty. Estrous cyclicity was monitored from 8-11 weeks of age. Offspring were killed on PND 7779 , with females killed during diestrus. Organs were weighed and examined histologically. The litter was considered the statistical unit in evaluations conducted during the lactation period. For offspring data collected after weaning, individual animals were considered the statistical unit. Statistical analyses included ANOVA, Fisher exact test, and Mann-Whitney $U$-test.

Because the purpose of this Expert Panel evaluation is to evaluate the effects of soy formula, discussion of results will focus on effects in groups fed the soy protein-containing versus soyfree diets and dietary effects on ethinyl estradiol responses. No effect of diet on maternal body weight gain during gestation or lactation was detected. No effect of diet on litter size was detected. On PND 2, male pups from the soy-free diet group weighed significantly less than males in the soy diet group. Offspring body weight gains on PND 10-12 in both sexes were significantly lower in the soy-free than the soy-diet group. Diet alone had no detected effect on absolute anogenital distance, but anogenital distance adjusted for body weight was slightly higher in females of the soy-free versus soy diet group. [Diet was said to interact with ethinyl estradiol effects on anogenital distance in females, but the type of interaction was not clear.] At the PND 21 necropsy, body weights of females in the soy-free groups were lower than in the soy diet group. Ovary weight relative to body weight was higher in the soy-free than the soy-diet group.

In offspring evaluated in adulthood, body weight gain on PND 21-42 was significantly reduced only in males from the ethinyl estradiol/soy-free diet group. Day of vaginal opening was significantly accelerated and body weight at vaginal opening was significantly lower in females 
from the ethinyl estradiol/soy-diet group. Body weight at preputial separation was significantly lower in the soy-free diet compared to the soy-diet group. Necropsies conducted in adult offspring showed that the soy-free compared to the soy-protein diet significantly increased relative pituitary weights in males, decreased absolute and relative pituitary weights in females, and increased absolute and relative adrenal weights in males. Ethinyl estradiol induced a significant reduction in absolute and relative ovary weight only in offspring of rats fed the soy diet. The most prominent effects in female offspring exposed to ethinyl estradiol were irregular estrous cycles and histopathologic changes in reproductive tissues as outlined in Table 36 . The number of rats with estrous cycle irregularities was statistically altered only in the soy-diet group, but the numbers were increased in both dietary groups exposed to ethinyl estradiol in utero. Incidence and severity of lesions in the ovary, uterus, vagina, mammary gland, and pituitary were greater in offspring of rats exposed to ethinyl estradiol and fed soy compared to soy-free diets.

The study authors concluded that typical estrogenic responses to ethinyl estradiol were enhanced by soy-bean-derived factors.

Strengths/Weakness: Strengths of the study were use of soy-free chow with similar nutritional contents as soy-containing chow, oral exposure route, determination of feed consumption, standardization of litter size (PND 10), and killing of adult females at the same stage of the estrous cycle. Phytoestrogens were measured in chow, but no methods and few data were presented. Weaknesses were the use of only seven animals/treatment group, and lack of determination of reproductive capability.

Utility (Adequacy) for CERHR Evaluative Process: The study is of limited utility based on the small number of animals used.

Mäkelä et al. (1990), support not indicated, examined prostate effects in offspring of rats fed soy-containing or soy-free diets. From 1 week before mating through gestation and lactation, Sprague-Dawley rats were fed a commercial rat diet containing 13\% soy (control group, $\mathrm{n}=$ 2 dams) or a soy-free, casein-based diet ( $n=3$ dams). Pups were weaned at 21 days of age to the commercial (soy-containing) diet. Offspring were killed at 10 weeks of age, and prostates were removed and weighed in 13 or 14 animals/group. $5 \alpha$-Reductase production was measured by determining levels of metabolites after incubation of prostates in media for $30 \mathrm{~min}$. Prostates were then fixed in Bouin fluid for sectioning and morphometric analysis. Data were analyzed by ANOVA followed by $t$-test with Bonferroni correction. Prostate weights were significantly higher [by $\sim \mathbf{4 0 \%}$ ] in offspring of rats fed the soy-free diet. Diet had no detected effect on prostate $5 \alpha$-reductase activity or volume density of epithelium, lumina, or intra-acinar stromal tissues.

Strengths/Weakness: Weaknesses of the study were the lack of comparison of chows, the small number of animals/group $(\mathrm{n}=2-3)$, and evaluation of only males.

Utility (Adequacy) for CERHR Evaluative Process: The study is not useful because of the small numbers of animals used and the very small number of endpoints examined.

Atanassova et al. (2000), supported by the European Center for the Ecotoxicology of Chemicals and Astra-Zeneca, examined the effects of neonatal exposure to weak and strong estrogens on pubertal spermatogenesis and long-term changes in the reproductive system of male rats. As part of this study, adult female Wistar rats were fed standard diets (15.5\% soy meal) or soy-free diets (soy substituted by fishmeal and cereal content increased from $64 \%$ to $78 \%$ ) for 3 weeks before mating and through mating, pregnancy, and lactation. Male offspring of rats fed soy-free diets were maintained on soy-free diets from weaning until termination. 
An unspecified number of males born to mothers on the soy-free diets received s.c. injections of $4 \mathrm{mg} / \mathrm{kg}$ bw/day genistein [purity not specified] in phosphate-buffered saline vehicle on PND 2-18, and that part of the study is discussed in the CERHR Expert Panel Report on Genistein (Rozman et al., in press). Males from the soy-free control group were compared to males in the standard diet control group. [The total number of rats treated was not stated, but 7-14 rats/group were evaluated.] On PND 18 and 25, rats were killed and testes were fixed in Bouin fluid. Testicular cell numbers and seminiferous tubule lumen formation were determined by standard point counting of cell nuclei. Apoptosis was assessed by DNA fragmentation detected by in situ DNA 3'-end labeling. Plasma FSH and inhibin B were measured by RIA and enzyme-linked immunosorbent assay (ELISA), respectively. In addition, mating and fertility were examined in adult rats (80-90 days old) by placing them in a cage with an unexposed female for 7 days. Statistical significance was determined by ANOVA.

Results and statistical significance for endpoints characterizing pubertal spermatogenesis in 18- and 25- day-old rats are listed in Table 37. The study authors noted that the increase in spermatocyte nuclear volume per Sertoli cell nuclear volume in rats fed soy-free compared to standard diets on PND 18 suggested that dietary soy retarded an aspect of pubertal spermatogenesis. For parameters also assessed on PND 25, the only significant effect that remained was the increase in spermatocyte nuclear volume per Sertoli cell nuclear volume in soy-free compared to standard-diet controls. Testis weights in adult rats (90-100 days old) from the soy-free group were significantly $(8 \%)$ higher compared to rats in the standard-diet group, and testis weights of rats in the genistein group were similar to those in the soy-free group. Animals in the soy-free control group were not mated.

In a larger study reported in this study, body weight, testis weight, and plasma FSH levels were compared in 24 litters from soy-free groups and 29 litters from standard-diet groups. Male rats were evaluated at 90-95 days of age. Rats in the soy-free group had significantly higher body $(5.7 \%)$ and testis weights (3.6\%) and significantly reduced plasma FSH levels (11.1\%).

The study authors concluded that "the presence or absence of soy or genistein in the diet has significant short-term (pubertal spermatogenesis) and long-term (body weight, testis size, FSH levels, and possibly mating) effects on males."

Strengths/Weakness: A strength of this study was the large numbers of litters examined $(\mathrm{n}=$ 24-29). Weaknesses included lack of comparison of dietary nutrients, examination of only males, and lack of determination of reproductive capability. Exposure through chow is a strength in its relevance to human exposure and a weakness in permitting only imprecise estimates of dose levels.

Utility (Adequacy) for the CERHR Evaluative Process: The endpoints examined in this study are of limited utility alone in determining developmental effects.

McVey et al. (2004b), of Health Canada, reported serum and testicular androgen levels in $\mathrm{F}_{1}$ rats exposed to a soy supplement in a multigenerational study in Sprague-Dawley rats. [The full report of the multi-generational study is not yet available (G. Cooke, personal communication, December 2, 2004).] Six diets were used in the study. The control diet was a soy-free casein-based diet (AIN93G) in which there were no detectable isoflavones by HPLC after $\beta$-glucuronidase digestion. A second diet was similar to the control diet but included a commercial soy-protein concentrate (Pro Fam 930) in place of casein. This diet was supplemented with a commercial soy-isoflavone concentrate (Nova-soy®) at four different levels of supplementation. The total isoflavone levels in the soy-containing diets were 31.7, $36.1,74.5,235.8$, and $1046.6 \mathrm{mg} / \mathrm{kg}$ feed [ppm]. Of the total isoflavones that were measured by HPLC after $\beta$-glucuronidase, 33-39\% was daidzein, 8-10\% was glycitein, and $52-59 \%$ was 
genistein. The authors noted that the range of isoflavone content in the diets represented a range of human consumption of soy isoflavones from the negligible amounts in North American diets to the high levels consumed by infants on soy formula. Diets were provided ad lib from 50 days of age to male and female rats. After 70 days on the diets, animals were mated to produce an $F_{1}$ generation, which was weaned on PND 21 to the same diet assignment as the parents. $F_{1}$ males were killed on PND 28, 70, 120, 240, or 360 for measurement of testosterone and dihydrotestosterone in serum and in homogenized testes using a commercial ELISA kit. ANOVA with post-hoc Tukey test was used for comparisons.

Based on feed consumption and body weight data, estimated $\mathrm{F}_{1}$ total isoflavone intakes in the six diet groups at PND 28 were $0,0.9,1.2,2.0,6.3$, and $29.5 \mathrm{mg} / \mathrm{kg}$ bw/day. On PND 120, estimated $F_{1}$ total isoflavone intakes were $0,0.9,1.0,2.1,7.3$, and $28.7 \mathrm{mg} / \mathrm{kg}$ bw/day. There were no detected treatment-related alterations in mean body weight or feed consumption at any time during the study. Testis weights were increased by about $30 \%$ in the PND 28 males on the Novasoy ${ }^{\circledR}$-containing diets (total isoflavone intakes $\geq 2.0 \mathrm{mg} / \mathrm{kg}$ bw/day, $\mathrm{n}=5$ or $6 /$ treatment group). Serum testosterone peaked in all groups at the PND 70 measurement, consistent with puberty in rats, and remained elevated at PND 120 in the groups exposed to the diets with the two highest isoflavone levels. No differences between groups were detected at any time point other than PND $120(n=12$ /group/time point except $n=6$ /group on PND 360). Serum dihydrotestosterone followed the same pattern, with PND 120 levels statistically higher in the two highest isoflavone groups than in the other groups. No significant alteration in the ratio between testosterone and dihydrotestosterone was detected at any time point. Intratesticular testosterone reached a peak on PND 120, at which time values in all soy-supplement groups seemed graphically higher than those in the control group but without evidence of dosedependency and with a statistical difference from control only for the second-highest isoflavone-diet group. Intra-testicular dihydrotestosterone (ng/g testis) seemed to show a numerical decrease on PND 28 [the only time point for which data were given] as dietary isoflavone concentration increased, but no statistically significant changes were identified by the study authors. [Trend test carried out by CERHR was significant at $\boldsymbol{P}=\mathbf{0 . 0 1 5}$.] The authors concluded that developmental exposure to isoflavones could alter testicular weight and androgen levels, although the mechanism for the apparent modulation of Leydig cell androgen production was not known.

Strengths/Weaknesses: Strengths of the study were determination of dietary isoflavone content, use of five different soy doses in chow, and monitoring of food consumption and soy intake. Weaknesses included examination of only males, lack of dose-response relationship for altered endpoints (e.g., plasma or testicular testosterone and dihydrotestosterone), lack of determination of reproductive capability, and lack of indication of the number of litters represented.

Utility (Adequacy) for CERHR Evaluation Process: This study is of low-to-moderate utility based on the limited usefulness of the endpoints.

McVey et al. (2004a), support not indicated, examined the effects of lifetime exposure to isoflavones on steroidogenic enzyme activities in rat testes. Throughout gestation and lactation, female Sprague-Dawley rats [number not reported] were fed one of six diets containing casein or soy as the protein source. The casein diet served as the control. The soy diets were alcohol-extracted to remove naturally occurring isoflavones and then fortified with Novasoy, an isoflavone supplement, at the isoflavone concentrations listed in Table 38. Male offspring were weaned on PND 21 and fed the same diets as their mothers. Male offspring (5-9/group) were killed on PND 28, 70, 120, 240, or 360. Enzyme activities were determined for 3 $\beta$ hydroxysteroid dehydrogenase, CYP17, 17 -hydroxysteroid dehydrogenase, and $5 \alpha$ reductase. Expression of messenger ribonucleic acid (mRNA) and protein was determined for 
3ß-hydroxysteroid dehydrogenase, CYP17, and P450 SCC, the mitochondrial cholesterol side chain cleavage enzyme involved in the first step of androgen production. Data were analyzed by linear and non-linear regression, ANOVA, and Tukey test. Enzymes activities declined with age in all dietary groups. As noted in Table 38, the only effects on enzyme activities occurred on PND 28 and included increased 3 $\beta$-hydroxysteroid dehydrogenase activity in rats fed diets containing isoflavones 235.6 and $1046.6 \mathrm{mg} / \mathrm{kg}$ diet and increased CYP17 activity in rats fed diets containing isoflavones $235.6 \mathrm{mg} / \mathrm{kg}$ diet. No differences were detected compared to controls at later time periods or in other enzyme activities. There were also no detected differences in mRNA or protein expression. According to the study authors, these results suggest that isoflavones at levels consistent with infant exposures alter testicular enzyme activities in rats during development.

Strengths/Weaknesses: Strengths of this study include the use of five different dose levels of soy, including doses with relevance to human exposure, and the measurement of isoflavone content of the diet. Weaknesses for this evaluation include the lifetime exposure to soy, the examination of only male offspring, the fairly small numbers of animals per group $(n=5-9)$, and the lack of consideration of litter effects. The endpoints evaluated (steroid metabolizing enzyme activities and mRNA and protein expression) are of little utility alone, but may provide mechanistic information useful in interpreting results from other studies.

Utility (Adequacy) for CERHR Evaluation Process: This study is of low utility based on inappropriate exposure time. It is also difficult to reconcile the results of this study with those reported earlier by this group using the same dietary conditions (McVey et al., 2004b). In their previous study, the authors showed that serum testosterone and dihydrotestosterone were slightly (but not significantly) decreased at PND 28 compared to casein-exposed controls and were increased at PND 120. However, in this study, enzyme activities were altered at PND 28 but not at later time points, including PND 120. Additionally, the enzyme activity changes reported in this study were not dose-related.

Robertson et al. (2002), supported by the National Health and Medical Research Council of Australia, examined the effects of a soy-based diet on aromatase-knockout mice. The knockout mice, which are unable to synthesize endogenous estrogens, are initially fertile, but at around 18 weeks of age, spermiogenesis is disrupted as a result of a post-meiotic defect. Groups of wild-type and aromatase-knockout mice were fed a diet that contained $10 \%$ soy meal or a soyfree diet. Total isoflavone levels were reported at $146 \mathrm{mg} / \mathrm{g}$ soy-based diet. The study authors stated that each type of diet was fed to mice beginning at birth. [It is not clear if the diets were fed to offspring and parents. Most likely, the parental mice were exposed because the authors stated that all knockout mice were fed the soy-free diet for 2 years before the study, with the exception of 10 mating pairs fed the soy-containing diet.] One set of wildtype and knockout mice ( $n=6-12 /$ group) fed either the soyfree or soy-containing diet and exposed during the same time period were killed at 14 weeks of age. A second set of wild-type and knockout mice fed the soy-free diet ( $\mathrm{n}=11-12$ /group) were killed at 1 year of age, and data obtained from these mice were compared with data obtained from mice fed the soycontaining diet in a previous study ( $n=6-8 /$ group). [The Expert Panel notes that 1-year-old animals from the two different diet groups were not exposed concurrently.] Parameters examined included terminal body weight, testis weight, testicular morphology, spermatogenic germ cells in the testis, and serum LH and FSH levels. Statistical analyses included ANOVA and least-squares significant difference test.

Terminal body weights were significantly higher in 14-week-old wild-type [24\% higher] and knockout mice [17\%] and in 1-year-old knockout [25\%] mice fed the soy-free versus soycontaining diet. Testis weight was not affected by diet or genotype. Qualitative evaluations of testes showed more pronounced disruption of spermatogenesis in 1-year-old mice fed the soy- 
free versus soy-containing diet [data not shown]. Quantitative testicular observations that were statistically significant are summarized in Table 39. Few and minimal effects were observed in 14-week-old mice. The study authors noted that at 1 year of age, knockout mice fed the soy-containing diet had increased spermatogenic cell numbers compared to knockout mice fed the soy-free diets. Compared to knockout mice fed soy-free diet, the knockout mice fed the soy-containing diet had significantly lower [40\%] serum FSH levels, but no significant effects of dietary exposure on LH levels in knockout mice were detected. The study authors concluded that low levels of dietary phytoestrogens exert biological effects on the testis that are independent of effects on the pituitary-gonadal axis.

Strengths/Weaknesses: Strengths are that chow was analyzed for isoflavone content and that the study used reasonably sized groups $(n=6-12)$. The lifetime exposure to soy and the use of only one dose level are weaknesses.

Utility (Adequacy) for CERHR Evaluation Process: This study is of low utility based on the inappropriate exposure period and use of only a single dose level of soy. However, this study did demonstrate that estrogen is required for normal spermatogenesis and that the phytoestrogens in soy can partially compensate for the inability to synthesize estrogen in aromatase-knockout mice.

Mäkelä et al. (1995b), supported by Turku University Foundation, Yrjö Jahnsson Foundation, and Emil Aaltonen Foundation, evaluated the anti-estrogenic effects of dietary soy in developing male Han-NMRI mice. Animals were given either a standard laboratory chow containing $7 \%$ roasted soybean meal or a commercial soy-free feed. Female mice were fed either the soy diet or the soy-free diet during pregnancy and lactation [n not given]. At weaning, half the males in each litter were continued on their dams' diet and half were switched to the opposite diet ( $n=27-29 /$ group). At 2 months of age, urethroprostatic blocks were harvested for dissection of seminal vesicles, coagulating gland, ventral prostate, and dorsolateral prostate. Relative weights of these organs and testes were recorded, and histopathology was evaluated on the reproductive organs of five animals/group. An additional experiment was carried out using males exposed during pregnancy, lactation, and thereafter to one of two diets (15-19/ group) with harvesting and examination of reproductive organs at 12 months of age. Other experiments described in this study used the same design (exposure during pregnancy, lactation, and thereafter), but also treated male pups with s.c. diethylstilbestrol on the first 3 days of life. Evaluations of the reproductive organs of these males were carried out at 2, 9, or 12 months of age ( $n=13-19 /$ group). Statistical analysis used the Student-Newman-Keuls multiple-range test, Fisher exact test, or ANOVA with post-hoc $t$-test. [The number of animals in the data tables were lower than the number of animals identified in the Methods section for some experiments.]

Coagulating gland and combined prostate lobe relative weights were increased in 2-month-old males fed a soy diet after weaning, after a soy-free diet given to the dam during pregnancy and lactation, when compared to animals exposed to soy during pregnancy and lactation and after weaning. The relative weight of the prostate lobes was also significantly higher than the comparable organ weights of males placed on a soy-free diet after weaning, regardless of which diet their dams had been fed. The increase in prostatic lobe relative weight in the group fed soy after being weaned from soy-free dams was 11-19\%. There were no detected body weight changes or accessory gland histologic alterations associated with soy feeding status. When males were evaluated at 12 months after exposure to the same soy-free or soy-containing diets as their dams received during pregnancy and lactation, relative reproductive organ weights were increased by soy feeding. The magnitude of the increase was $40 \%$ for ventral prostate, $60 \%$ for coagulating gland, $63 \%$ for dorsal prostate, $35 \%$ for seminal vesicles, and $20 \%$ for testes. No body weight changes or histological differences associated with soy feeding status 
were detected. In neonatally estrogenized males evaluated at 2 months of age, soy feeding of dams and offspring was associated with a $46 \%$ increase in relative weight of the coagulating gland and an $81 \%$ increase in relative weight of dorsolateral prostate, without a change in body weight. There was a soy-associated decrease in the proportion of animals with severe dysplasia when the accessory reproductive organs were histologically evaluated at 9 months of age (3/10 compared to 8/10). The authors also stated that the incidence of severe dysplasia was decreased by soy feeding at 12 months, although the difference between groups was not statistically significant (7/11 compared to $12 / 14[\boldsymbol{P}=\mathbf{0 . 7 6}$, Fisher exact test $])$.

The authors concluded that there was an anti-estrogenic effect of feeding a soy diet during male development because of amelioration of diethylstilbestrol-associated prostatic growth inhibition and because of a delay in diethylstilbestrol-associated prostatic dysplasia. There was no conclusion concerning the differential effects of soy exposure of dams during pregnancy and lactation or soy exposure of offspring after weaning.

Strengths/Weaknesses: A strength was that the two diets were analyzed and found to be similar in protein, vitamin, and mineral content. In addition, urinary isoflavones and lignans were measured. Weaknesses were lack of indication of the number of animals/group and apparent lack of consideration of the litter as experimental unit. Exposure through chow is a strength in its relevance to humans and a weakness in permitting only imprecise estimates of dose levels.

Utility (Adequacy) for CERHR Evaluation Process: The study is of low utility based on lack of experimental details.

Sharpe et al. (2002), support not indicated, fed male marmoset offspring a soy infant formula (Wysoy®) or a cow-milk formula (SMA Gold®). The animals included 13 pairs of co-twins assigned to different formulas to permit paired statistical tests and four additional animals. At 4-5 days of age, the marmoset offspring were intermittently separated from their mothers by a wire mesh divider, permitting mothers and young to be within sight of one another. The periods of separation included the 8-hr work-day on weekdays and about $2 \mathrm{hr}$ on weekends. During this time, the infants remained with their fathers and siblings in the family cage. Because marmoset young typically receive care (other than feeding) from their fathers and older siblings, these periods of separation did not seem to distress either the infants or the mothers. During the periods of separation, infants were hand-fed their assigned formulas using a soft tube connected to a syringe. The formulas were prepared according to the instructions for human infant feeding and were administered in quantities limited only by the marmoset infant's appetite. Animals were formula-fed three or four times on weekdays and once or twice on weekend days. Except for periods of separation, the marmoset infants were caged with their mothers and permitted to nurse. Based on the composition of the formulas and the amount consumed, the investigators estimated that the marmoset infant intake of isoflavones during the 5-6 weeks of the study was $1.6-3.5 \mathrm{mg} / \mathrm{kg}$ bw/day. On PND 18-20 and 35-45, infant blood was taken for plasma testosterone determination by ELISA. In seven pairs of twins, the second blood sample was obtained by cardiac puncture after the animals were killed for evaluation. The earlier blood sample in these animals and both blood samples in the animals that remained alive were obtained from the femoral vein. Testes and pituitaries were removed from the animals killed on PND 35-45. Testes were weighed after fixation in Bouin fluid. Testes were sectioned and evaluated for Sertoli and germ cell number, Leydig cell number per testis using immunostaining for $3 \beta$-hydroxysteroid dehydrogenase, and intensity of staining for $17 \alpha-$ hydroxylase/ $\mathrm{C}_{17-20}$ lyase. Pituitaries were fixed in Bouin fluid, sectioned, and immunostained for the $\beta$-subunits of LH and FSH. Statistical comparisons were made using paired $t$-tests. 
No differences were detected between treatment groups in body weight at the beginning or the end of the treatment period. No difference in formula intake were detected between the treatment groups. Plasma testosterone was similar between the treatment groups on PND 1820, but was lower in the soy formula-fed group on PND 35-45 when analysis was restricted to the 13 pairs of twins (mean \pm SD $1.3 \pm 2.1 \mathrm{ng} / \mathrm{mL}$ compared to $2.8 \pm 3.9 \mathrm{ng} / \mathrm{mL}$ in the cow-milk formula-fed group, $P=0.004$ by paired $t$-test on log-transformed data). The proportion of 35 45-day-old animals with plasma testosterone $<0.5 \mathrm{ng} / \mathrm{mL}$ was $1 / 15$ in the group given cowmilk formula and $12 / 15$ in the group given soy formula $(P<0.001$, Fisher exact test). Testis weights were comparable between the two groups, as were Sertoli and germ cell numbers/ testis. Leydig cells/testis were increased $74 \%$ in the soy formula-fed group at 35-45 days of age. No differences by treatment group were detected in intensity of staining for $17 \alpha-$ hydroxylase $/ \mathrm{C}_{17-20}$ lyase in testis sections and numbers of cells immunopositive for $\beta$-subunit LH or FSH in pituitary sections. Because blood concentrations of LH and FSH cannot be reliably measured in the marmoset, the authors could not determine whether the decrease in plasma testosterone was due to an effect of soy constituents on the pituitary or on the Leydig cell, but they believed the decrease in plasma testosterone to be potentially important, particularly in light of the normal increase in testosterone that occurs in neonatal primates, including humans.

Strengths/Weaknesses: Strengths of this study included use of a non-human primate species, appropriate exposure period, exposure to soy formula, and adequate numbers of animals/group. A weakness is that because animals were allowed to nurse, total soy exposure was unknown. Specifically, maternal chow was not described and possible exposure occurring through mother's milk was unknown. In addition, only one soy formula dose was used.

Utility (Adequacy) for CERHR Evaluation Process: The study is of low-to-moderate utility based on appropriateness of exposure period and species used. The endpoints are of limited utility alone in determining reproductive effects, but may be helpful in interpreting results from other studies.

Tan et al. (2006), funded by the U.K. Medical Research Council, extended the study of Sharpe et al. (2002) by describing outcomes in the seven marmoset twin pairs that were continued to 120-138 weeks of age. The treatment regimen was as described above. At the end of the formula-feeding period (35-45 days of age), these animals were maintained in their family groups until 80-140 weeks. Thereafter, each male was caged singly with an adult female and any resulting offspring until termination. Between 40 and 120 weeks of age, plasma was sampled every 10 weeks for measurement of testosterone by RIA. After death, testis, epididymis, prostate, seminal vesicles, thymus, spleen, and pituitary were dissected, fixed in Bouin fluid, and weighed. The right testis was sectioned, infiltrated with resin, and stained with Harris hematoxylin for enumeration of Sertoli cells. The remaining tissues were embedded in paraffin and sectioned for light microscopy. Immunostaining for $3 \beta$-hydroxysteroid dehydrogenase was used to quantify Leydig cell number, nuclear diameter, and volume. Cytoplasm staining for 3 $\beta$-hydroxysteroid dehydrogenase was expressed as relative volume of the testis and multiplied by testis weight for an estimate of Leydig cell volume. Stereologic methods were used to estimate seminiferous epithelium and luminal volumes. Statistical analysis was carried out using paired $t$-tests.

No effects of formula type were detected on body weight or terminal weights of thymus, spleen, prostate, seminal vesicles, or pituitary, and penile length was comparable between the feeding groups. Testis weight was $14 \%$ higher in the soy formula-fed than the cow-milk formula-fed group, and in six of seven twin pairs, testis weight was higher in the soy formula-fed twin than in its co-twin. A slightly lower testis weight in one of the cow-milk formula-fed twins compared to its co-twin was attributed to Sertoli-only tubules, considered most likely to be a result of 
blockage of the excurrent duct system. The timing of puberty onset, determined by an increase in plasma testosterone, was not identified as altered by infant feeding method, and no significant group differences in plasma testosterone were detected at any time point. Two of the soy formula-fed marmosets had plasma testosterone levels that were consistently at the lower end of the normal range. All but one of the marmosets produced young; the animal that did not produce young was one of the soy formula-fed animals with consistently low-normal testosterone plasma levels. No determination was made of the female partner's fertility status, precluding the conclusion that this male was infertile. There was no formal assessment of mating behavior.

Except for the one cow-milk formula-fed marmoset with Sertoli-only tubules, testicular histology was normal. Sertoli and Leydig cell numbers/testis were higher after soy-formula feeding in each of the 7 individual twin comparisons, and group means were significantly higher for both cell types in the soy formula-fed animals. Leydig cell numbers were increased to the greatest degree (55\% and $214 \%$ compared to the control co-twin) in the two soy formulafed animals with the consistently low-normal plasma testosterone levels. The volumes of the seminiferous epithelium and of the tubular lumina were described as higher in the soy formulafed group; however, there was substantial variability among twin pairs, and the group means were not statistically different.

The authors attributed the higher testis weights in the soy formula-fed group to a presumed larger number of germ cells secondary to the large number of Sertoli cells. They concluded that these results were generally reassuring in showing normal reproductive outcome after soy formula feeding during infancy; however, they postulated that the soy-associated increase in testis weight and Leydig cell numbers may have represented a state of compensated Leydig cell failure. This possible Leydig cell failure was most evident in the two animals with lownormal plasma testosterone levels that had the largest increase in Leydig cell numbers compared to their co-twins. The authors stated that because their design did not permit $100 \%$ formula-feeding, soy-formula intake of their marmosets would have been only $40-87 \%$ of the intake of a human infant exclusively fed soy formula. The exclusive feeding of soy infant formula in humans, they suggested, might be more likely to produce adverse reproductive effects.

Strengths/Weaknesses: The use of a co-twin design to minimize the number of animals used and to control for litter effect and large inter-animal variability is a potential strength of the design; however, no data were presented to verify the assumption that male twins are more comparable for reproductive parameters than unrelated males of the same age or to demonstrate that the rate of maturation is the same in co-twins. The variance of untreated co-twins compared to other untreated marmosets of similar age and body weight was not presented. Maintaining family groups during treatment to minimize stress is a strength. Marmosets were exposed orally to soy formula during the neonatal period with feeding on demand, which is relevant to human infants. The authors estimated that isoflavone intake for soy formula-fed males was 40-87\% of 4-month-old human infants maintained on soy formula; thus, exposure levels were realistic. When determining Leydig cell numbers, the authors based the number of fields counted per animal on obtaining a percentage standard error value of $<5 \%$, which is a strength, as is reporting the detection level and intra- and inter-assay coefficients of variation for testosterone assays. When determining testosterone levels, sample from co-twins were processed at the same time. Offspring in both treatment groups reportedly consumed similar amounts of formula. The decrease in plasma/serum testosterone levels and increase in Leydig cell numbers with soy formula treatment agreed with results seen at 35-40 days of age in soy formula-fed marmosets in the previous Sharpe et al. (2002) study. 
The study compared only one concentration of soy formula, so it is not possible to evaluate dose-response relationships. As is common in primate studies, the sample sizes were small, but the use of seven co-twins disagrees with the original study (Sharpe et al., 2002) in which only six sets of co-twins were maintained to adulthood (plus four male non-twins). It would have been useful if the authors had presented some historical control data on untreated control laboratory values for marmosets. As the data are presented, it is not possible to determine how the testicular parameters in cow-milk formula-fed controls relate to typical marmoset values, and the variances for measured parameters were not identified. Marmosets were returned to their biological mother for $16 \mathrm{hr} /$ day on weekdays and $22 \mathrm{hr} /$ day on weekends; thus, breast feeding during this interval may have masked some effects of an entirely soy-based diet. There were no data on blood levels of genistein or daidzein, so it is difficult to determine how marmoset kinetics might differ from those of human infants. The authors used values reported previously of aglycone/L formula to estimate marmoset exposures, which is a weakness. It is not clear why the authors fixed the reproductive organs before collecting organ weights. It is not possible to determine whether the authors controlled for diurnal variation in their measurements of hormones by collecting all samples at the same time each day. Fertility in co-habited females was not proven. Co-twin 7 had a pronounced incidence of seminiferous tubules that contained only Sertoli cells. The authors hypothesized that this abnormality was due to partial blockage of the efferent ducts or rete and was unlikely to be related to treatment; however, they did not exclude this animal (or its co-twin) from other testicular measures. The authors reported that soy formula attenuated the neonatal rise in testosterone (Sharpe et al., 2002); however, marmosets seem to be more resilient to disturbances in neonatal testosterone levels than humans ( $\mathrm{Li}$ et al., 2005). The authors hypothesized that the soy formula-treated males may exhibit a degree of "compensated Leydig cell failure" based on increased Leydig cell numbers with normal testosterone levels. The authors were unable to measure marmoset serum LH levels, but hypothesized that elevations of gonadotropin levels (both LH and FSH) could provide an explanation. According to Li et al. (2005), it is difficult to extrapolate marmoset data to humans because marmosets differ markedly in some areas of testicular development, including the lack of a regular $\mathrm{LH} / \mathrm{LH}$ receptor system, the presence of a positive feedback loop for $17 \beta$-estradiol in normal male marmosets, and a generalized steroid resistance due to high circulating levels of testosterone, estrogen, and other steroids.

Utility (Adequacy) for CERHR Evaluation Process: This study of limited utility in the evaluation process.

Anthony et al. (1996), funding not indicated, presented a limited examination of hormonal levels and reproductive organ weights in rhesus monkeys (Macaca mulatta) fed soy protein diets as part of a study examining the effects of soy isoflavones on cardiovascular risk. Peripubertal male (1.3-2.1 years old) and female (1.3-4.1 years old) monkeys were fed diets containing casein and lactalbumin as the source of protein for 3 weeks. The monkeys were then stratified randomly according to age and cholesterol levels. For a 24-week period, one group of seven females and five males was fed a soy-based diet containing $1.27 \mathrm{mg}$ genistein and $0.42 \mathrm{mg}$ daidzein per g soy protein. The diet resulted in isoflavone intake of $9.41 \mathrm{mg} / \mathrm{kg}$ bw. [Assuming that genistein and daidzein were the only isoflavones present, intake of each respective isoflavone would be $\sim 7$ and $2 \mathbf{~ m g} / \mathbf{k g ~ b w}$.] A second group of seven females and six males was fed a diet in which the soy protein was alcohol-extracted to remove most of the isoflavones. Levels of isoflavones in the isoflavone-reduced diet were $0.121 \mathrm{mg}$ genistein and $0.052 \mathrm{mg}$ daidzein per $\mathrm{g}$ soy protein. The reduced-isoflavone diet provided an isoflavone intake of $0.97 \mathrm{mg} / \mathrm{kg}$ bw [genistein $\sim 0.7 \mathrm{mg} / \mathrm{kg} \mathrm{bw}$ and daidzein $\sim 0.3 \mathrm{mg} / \mathbf{k g ~ b w}$ ]. As part of the cross-over study design, each group of monkeys received one of the diets for 24 weeks and was then switched to the opposite diet for 24 weeks. Blood levels of 17 $\beta$-estradiol, testosterone, dehydroepiandrosterone sulfate, and thyroxine were measured by RIA, and sex hormone-binding globulin was measured by a saturation assay. It seems that results were 
pooled for animals of the same treatment groups regardless of exposure period. After the second exposure period, monkeys were killed and prostate, testis, and uterus weights were obtained. Data were analyzed by ANCOVA and paired $t$-tests. The presence of dietary isoflavones in the soy diet had no detected effect on hormone levels or reproductive organ weights. The authors reported the intact isoflavone protein had favorable effects on plasma lipid and lipoprotein concentrations. [As noted by study authors, all male monkeys were prepubertal at the start of the study and some reached sexual maturity during the study. Results could have been affected by imbalances in male sexual maturity.]

Strengths/Weaknesses: A strength of this study was use of rhesus monkeys, which is presumably one of the most relevant species for humans. The cross-over design allowed for measurements from the same animal on both diets (soy-based and alcohol-extracted), which is valuable given the small sample sizes. Monkeys were randomized based on age and the ratio of total plasma cholesterol to HDL cholesterol, one of the primary confounders in examining the soy effects on plasma lipid and lipoprotein concentrations. Diets were kept frozen until needed, which may have improved test material stability. Levels of genistein and daidzein were determined in both the soy-based and alcohol-extracted soy protein. Proteins were also analyzed for the presence of $\beta$-sitosterol. Samples were analyzed in duplicate. Statistical analyses seemed appropriate with analyses for main effects of treatment and period as well as any interaction between those terms. Some weaknesses were also noted. As is often the case in studies using monkeys, this study contained small sample sizes $(n=14$ females and 13 males at study start). Results were pooled for all animals receiving the same diet, regardless of differences in age or other factors. After the second 24-week exposure, animals were killed and reproductive organ weights were measured. It was assumed that the endpoints measured reflected the last diet to which the animals were exposed (i.e., that there were no long-term effects of soy-based diet because both groups had received this diet before necropsy). It was not possible to control for differences in the state of sexual maturity of the animals at the beginning versus the end of the study, making it unclear whether differences in hormone measurements or reproductive organ weights were masked. Data on these parameters and their variances were not provided. Furthermore, there was no evidence that the authors controlled for diurnal variation in their measurements of hormone levels. Despite attempts to control dietary components, the authors noted that "differences in the concentration of the protein in the products somewhat affected the percent of energy as protein, fat and carbohydrate," among other factors that could influence study outcome.

Utility (Adequacy) for CERHR Evaluative Process: This study is not useful in the CERHR evaluation process.

3.2.2 Mammary development/carcinogenesis-Hakkak et al. (2000), funded by the USDA, examined the effects of diet containing soy or whey protein on the development of dimethyl benzanthracene-induced mammary tumors in rats. Breeder Sprague-Dawley rats were fed AIN-93G diets in which soybean oil was replaced by corn oil and the protein source was either casein, whey, or soy-protein isolate. The soy diet contained isoflavones $430 \mathrm{mg} / \mathrm{kg}$ diet, specifically, genistein $276 \mathrm{mg} / \mathrm{kg}$ diet and daidzein $132 \mathrm{mg} / \mathrm{kg}$ diet. The study authors estimated that a $333 \mathrm{~g}$ rat eating $25 \mathrm{~g}$ diet/day would be exposed to genistein at $20.4 \mathrm{mg} / \mathrm{kg}$ bw/day. Rats from the same dietary groups were bred to produce $F_{1}$ offspring, and on maturity, the $F_{1}$ offspring from the same dietary groups were bred to rats from different litters to produce $\mathrm{F}_{2}$ offspring. [The number of rats bred was not stated for any generation, and it is not known how many litters were represented in $\mathbf{F}_{1}$ rats selected for breeding.] At weaning, the $F_{1}$ and $F_{2}$ offspring were fed the same diets as their dams and continued to receive the diets throughout their lifetimes. [Based on a body weight figure presented in the study, it was estimated that a weanling (21-day-old) rat weighs $50 \mathrm{~g}$. The USEPA (1988) assumption 
for food intake in a weanling female Sprague-Dawley rat is $0.150 \mathrm{~kg} / \mathrm{kg} \mathrm{bw} / \mathrm{day}$. Therefore total genistein intake in weanling rats was estimated at $\sim 830 \mathrm{mg} / \mathrm{kg}$ bw/day.] At 50 days of age, $\geq 19 \mathrm{~F}_{1}$ rats/group [inconsistent values reported for number of whey-fed rats in various tables] and $\geq 31 \mathrm{~F}_{2}$ rats/group were gavaged with dimethyl benzanthracene 80 $\mathrm{mg} / \mathrm{kg}$ bw and the development of palpable mammary tumors was assessed. Rats were killed when all rats from the casein diet had at least one palpable mammary tumor. Tumors were examined, fixed in buffered formalin, and sectioned. Statistical analyses included ANOVA, Kaplan-Meier analysis, Wilcoxon test, Fisher exact test, and Kruskal-Wallis test.

Beginning at 8 weeks of age, body weight gain in both generations of casein-fed rats was slightly but significantly higher than body weight gain in the soy-or whey-fed rats. Vaginal opening was significantly accelerated by 1 day in the soy-fed compared to casein-or whey-fed rats (PND 37 vs. PND 38). No differences were observed for relative organ weight, estrous cycle, successful breeding, sex ratio, or litter size [data not shown]. Results for mammary carcinogenesis are out-lined in Table 40. Latency, in terms of the number of post-treatment days for $50 \%$ of rats to develop at least one tumor, was significantly increased in both generations of the soy- and whey-fed rats compared to casein-fed rats. Time course for tumor development was accelerated in the casein-fed rats, as noted by a significantly lower percentage of soy- and whey-fed rats with tumors at the time when $100 \%$ of casein-fed rats had developed a tumor. Tumor multiplicity (numbers of tumors/tumor-bearing rat) was significantly lower in both generations of whey-fed rats compared to casein-fed rats. There were no other significant differences in tumor parameters. The study authors concluded that soy-rich diets reduce dimethyl benzanthracene-mammary tumor incidence by about $20 \%$ in rats and that whey seems to be twice as effective as soy products in reducing tumor incidence and multiplicity.

Strengths/Weakness: A strength of the study is that rats were fed AIN-93 diets with casein, soy, or whey as the sole protein source for two generations. Dietary isoflavone, genistein, and daidzein concentrations were analytically determined. Animals were exposed through diet, a relevant route of exposure. Mammary tissue was examined by a pathologist who was blind to treatment group. In the statistical analyses, day of measurement was included to account for multiple measures per animal and interactions between diet and day were examined. Offspring were exposed to a soy-containing diet across critical windows of development. Dose levels were reportedly similar to those ingested by a 4-month-old infant consuming soy-based infant formula. A weakness is inclusion of only one dietary level of soy, which does not allow for evaluation of dose-response relationships. There were no analytical data presented for the stability of genistein in the diets, and no information as to how often diets were replenished. The authors did not state how many animals were bred in the parental or $\mathrm{F}_{1}$ generation or how many litters were produced. It is not known whether litter effect was controlled (i.e., selection of one male and one female/litter for mating of the next generation and use of litter-based analyses, such that animals from the same litter represented an $n$ of 1 ). Data for many endpoints (relative organ weights, estrous cycle, fertility, sex ratio, litter sizes) were not presented. The soy effect on lowering tumor incidence was only statistically identified in one of two experiments described in this study. Details were not provided for the assignment of animals into treatment groups (i.e., approximately equal mean body weights and variances across groups). It would have been useful if the authors had reported body weight at the time of puberty onset because a 1-day acceleration in vaginal opening in the soy-fed females likely occurred in animals at lower body weights than in casein controls. Furthermore, the mean age at vaginal opening in the female Sprague-Dawley rats (38 days in casein-fed controls) is later than the value reported in many other publications (e.g., 33.4 days, range $=31.6-35.1$ days) (Clark, 1999).

Utility (Adequacy) for CERHR Evaluative Process: This study is not useful in the CERHR evaluation process. 
Rowlands et al. (2002), funded by the USDA, examined the effects of a soy-protein isolate diet on mammary development in rats. Beginning on GD 4 [day of plug not specified], 10 pregnant Sprague-Dawley rats/group were randomly assigned to receive one of three AIN-93G diets containing soy protein isolate, whey, or casein as the protein source. The diets were equivalent in calories, protein, and amino acid content. [A statement was made that the animals were exposed throughout their lifetimes, and it was therefore assumed that the animals were fed the assigned diets through the remainder of gestation and the entire lactation period.] Offspring were weaned to the same diets as their dams. On PND 21, 33, and 50, one female/litter/group ( $\mathrm{n}=10 /$ time period) was killed for examination of mammary gland morphology. The same diets and exposure protocol were used in a second experiment in which offspring were selected from five to seven litters and were weaned to the same diets as their mothers. Around PND 48-51, one female/litter/group ( $n=5-7 /$ group) was gavaged with sesame oil and killed $24 \mathrm{hr}$ later in metestrus. Mammary glands were removed for examination of cell proliferation and expression of $\mathrm{ER} \alpha, \mathrm{ER} \beta$, and progesterone receptor. The litter was considered the statistical unit in analyses that included ANOVA and StudentNewman-Keuls multiple comparison test.

Mammary gland area in rats of the soy diet group was 36-38\% larger than in rats of the casein and whey protein groups on PND 50. This effect was not observed on PND 21 or 33. A fourfold $(75 \%)$ decrease in terminal end bud density was identified in rats of the soy and whey protein diet groups compared to the casein diet group on PND 50, but the effect did not attain statistical significance. No significant differences in alveolar or lobuloalveoli bud density were detected. Terminal end bud cells expressing progesterone receptor were increased by $34 \%$ in the soy protein compared to the casein diet group at 48-51 days of age, but this effect was not seen in lobular cells. No significant effects of the soy protein isolate diet on proliferation or expression of ER $\alpha$ or ER $\beta$ in terminal end buds or lobular cells were detected. The study authors concluded that the soy protein isolate diet stimulated mammary gland differentiation.

Strengths/Weakness: A strength was that rats were fed AIN-93 diets with casein, soy protein isolate or whey protein hydrolysate as the sole protein source. The authors controlled for litter effect, using either one female pup/litter for an endpoint or using the litter as the unit of analysis. The methods for scoring mammary whole mounts and immunohistochemistry samples were clearly described. The authors controlled for stage of estrous cycle when proliferation index was determined in mammary glands. A negative control (omitting the primary antibody) was included in immunohistochemistry experiments to verify receptor populations. Animals were exposed in the diet, a relevant route of exposure. The statistical analyses were appropriate. Offspring were exposed to a soy-containing diet across several critical windows of development and evaluated at $\sim 50$ days of age. A weakness is that the study included only one dietary level of soy, which does not allow for evaluation of dose-response relationships. Blood levels of phytoestrogens (e.g., genistein) were not measured. The authors did not specify whether the sperm-positive day was designated as GD 0 or 1 . There were no analytical data presented for the stability of genistein in the diets and no information as to how often diets were replenished. Dietary levels of genistein and daidzein were not specified [perhaps these levels were the same as Hakkak et al. (2000)]. No data on body weight were presented. Sample sizes were likely insufficient for endpoints such as mean number of terminal end buds $/ \mathrm{mm}^{2}$, given that a 75\% decrease was not statistically different. Details were not provided for the assignment of animals into treatment groups (i.e., approximately equal mean body weights and variances across groups).

Utility (Adequacy) for CERHR Evaluative Process: This study is not useful in the evaluation process. 
Simmen et al. (2005), supported by USDA, examined the effects of soy diet on chemically induced mammary tumorigenesis. On GD 4 (day of plug not stated), Sprague-Dawley rats were assigned randomly to groups fed AIN-93G diets containing either casein or soy-protein isolate as the sole protein source. [Based on numbers of pups reported later in the study, it seems that 10 dams/group received each diet.] Dams were allowed to litter, and pups from the same treatment groups were pooled. Five pups/sex/group were randomly placed with dams from the same treatment groups. The 50 female pups/group were weaned on PND 21 (day of birth not defined) and fed the same diets as their dams. On PND 50, female offspring were i.v. injected with $50 \mathrm{mg} / \mathrm{kg}$ bw $N$-methyl- $N$-nitrosourea. Rats were weighed weekly, palpated twice/week for mammary tumors, and killed 115 days after $N$-methyl- $N$-nitrosourea treatment. Mammary tissues were subjected to immunohistochemical analyses to determine proliferative cell nuclear antigen and apoptosis. RNA was isolated from mammary tissues and reverse transcription-polymerase chain reaction was used to determine expression for a number of genes involved in cell proliferation, cell differentiation, and apoptosis. Serum estrogen [not otherwise identified] and progesterone levels were measured. MCF-7 breast cancer cells were incubated in sera obtained from each diet group to measure apoptosis. Data were analyzed by ANOVA or Student $t$-test.

Compared to rats fed the casein diet, rats fed the soy-protein isolate diet had increased mean \pm SEM tumor latency $(93.0 \pm 4.3$ vs. $78.0 \pm 3.9$ [unit assumed to be day]; $P<0.05)$ and decreased incidence of rats with at least one tumor $(57.4$ vs. $83.3 \%$; $P<0.01)$. Rats fed the soy-protein isolate diet had a lower incidence of ductal carcinoma in situ ( 42 vs. $64 \% P=0.08$ ) but a higher incidence of infiltrating ductal carcinoma (55 vs. 33\%; $P=0.086$ ). Diet had no detected effect on cell proliferation or apoptosis. Progesterone receptor and HER-2/neu (involved in cell proliferation) gene expression were increased in rats fed the soy-protein isolate diet. Serum progesterone levels were $\sim 7$ times lower in rats fed the soy-protein isolate diet, but there was no detected effect on serum estrogen level. Apoptotic cells in an MCF-7 culture were modestly but significantly increased after incubation with sera from rats fed the soy-protein isolate compared to the casein diet [data not shown]. The study authors concluded that lifetime exposure to soy-protein isolate protected rats from $N$-methyl- $N$-nitrosourea-induced mammary carcinogenesis and altered signaling pathways, including progesterone receptor and HER-2/ neu.

Strengths/Weakness: It is a strength that the amino acid content of the diets was equalized; however, only one dose level of soy was used and only female rats were evaluated. Soy was given over a lifetime, making the study difficult to interpret for the current evaluation.

Utility (Adequacy) for CERHR Evaluative Process: This study is not useful for the evaluation process due to the lifetime exposure to soy and the focus on tumor detection as a primary endpoint.

3.2.3 Brain structure/behavior-A number of studies examined the effects of soy diet on nervous system development or behavior of rats. The studies are presented in order of those focusing on brain structure or chemistry followed by those focusing on behavior. There is also a study in rhesus monkeys.

Taylor et al. (1999), supported by the National Science Foundation and Brigham Young University, examined the effects of a phytoestrogen-containing diet on fetal calbindin levels in the medial basal hypothalamus and preoptic area of the brain. Pregnant Sprague-Dawley rats were fed a phytoestrogen-containing diet $(n=4)$ or phytoestrogen-free diet $(n=2)$ on GD $0-20$ (day of plug = GD 0). The phytoestrogen diet (phyto-200) contained phytoestrogens 200 $\mu \mathrm{g} / \mathrm{g}$ diet (genistein $95 \mu \mathrm{g} / \mathrm{g}$, glycitein $17 \mu \mathrm{g} / \mathrm{g}$, and daidzein $82 \mu \mathrm{g} / \mathrm{g}$ ). [The protein source of the phyto-200 diet was assumed to be soy; the protein source for the phytoestrogen- 
free diet was not given.] Diets contained the same percentages of protein, fat, and carbohydrates. Rats were killed on GD 20. Fetuses were sexed, weighed, and evaluated for anogenital distance ( $n=27-30$ /sex in the phyto-200 group and 15-16/sex in phytoestrogenfree group). The medial basal hypothalamus and preoptic area brain regions were collected from greater than or equal to five fetuses/sex/litter/dietary group and pooled according to sex and dietary group. Medial basal hypothalamus and preoptic area calbindin levels were measured by Western blot. Data were analyzed with ANOVA, followed by pair-wise Tukey comparison. No significant effects of diet were detected on feed or water intake during gestation or on litter size [data not shown]. No effects of diet on anogenital distance and fetal body weight were detected. Calbindin protein expression in the medial basal hypothalamus and preoptic area was significantly higher [by $\sim \mathbf{4 0 \%}$ ] and more similar to male values in females fed the phytoestrogen-free compared to the phyto-200 diet. Diet had no effect on calbindin protein expression in males. The study authors concluded that the data have far-reaching implications regarding possible influence of dietary phytoestrogens on fetal medial-basal hypothalamus and preoptic-area calbindin levels.

Strengths/Weakness: A strength was that animals were exposed in the diet, which is a relevant route of exposure. Diets were analyzed by HPLC to determine concentrations of the phytoestrogens genistein and daidzein. Western blot data were obtained from six immunoblots. Specificity of the calbindin antibody was verified by demonstrating a lack of binding to other calcium-binding proteins. After the chemiluminescence assay, blots were stained with India ink to ensure equal protein loading and efficient transfer. For immunohistochemistry, a negative control was included using adjacent tissue sections with pre-immune serum or a buffer-only control (without the primary antibody). A weakness was that the study included only one dietary level of soy, which did not allow for a comparison of dose-response relationships. The stability of the phytoestrogen-containing diets was not reported. Sample sizes were too small to be meaningful. Fetuses within the same litter exposed to phytoestrogencontaining or phytoestrogen-free diet in utero were not independent samples. Thus, sample sizes were $\mathrm{n}=4$ for pregnant dams given the phytoestrogen-containing diet and $\mathrm{n}=2$ for dams given the phytoestrogen-free diet. These sample sizes were too small to make any conclusions. Furthermore, the authors did not control for litter effects as they reported body weight and anogenital distance measurements in $\geq 15$ animals/group. Medial basal hypothalamus and preoptic area of the brain were collected from $\geq 5$ males and $\geq 5$ females/litter and were pooled by sex and dietary group, thus suggesting that not all litters were equally represented in the samples analyzed. Volumes of the medial basal hypothalamus and preoptic area of the brain were not measured. Blood levels of genistein/daidzein were not measured. The authors did not discuss how these data relate to human hypothalamic development.

Utility (Adequacy) for CERHR Evaluative Process: This study is not useful in the CERHR evaluation process.

Lephart et al. (2001), supported by the National Science Foundation and Brigham Young University, examined the effects of lifetime phytoestrogen exposure on anteroventral periventricular nucleus structure, aromatase level, and prostate weight in rats. Long Evans rat offspring used in this study were born to dams (at least 8/group) fed a diet containing phytoestrogen glycosides $600 \mu \mathrm{g} / \mathrm{g}$ diet (phyto-600) or a phytoestrogen-free diet throughout the gestation and lactation period. [Distribution of offspring by litter was not discussed.] According to a reference provided in this study (Lund et al., 2001a), protein sources were soy for the phyto-600 diet and fish meal for the phytoestrogen-free diet. Diets were reported to have equivalent amounts of protein, amino acids, carbohydrates, fats, vitamins, and minerals. $F_{0}$ breeders and $F_{1}$ offspring were examined in a series of experiments. Data were analyzed by ANOVA followed by Bonferroni post-hoc comparison. 
Experiment 1 examined effects in male and female breeders that had been exposed only during adulthood. Levels of phytoestrogens in brains of adult male breeders are reported in Section 2.1.2. In Experiment 2, offspring were weaned at 30 days of age to the same diets as their dams. Body and prostate weights were measured at 75 days of age in 7-8 rats/group. Compared to rats fed the phytoestrogen-free diets, rats in the phyto-600 group had significantly lower body weight [17\% lower], absolute prostate weight [31\% lower], and relative (to body weight) prostate weight [17\% lower]. Brain aromatase activity in the anteroventral periventricular nucleus area was measured at 110 days of age $(n=6 / \mathrm{sex} /$ group $)$ and reported to be unaffected by diet.

In Experiment 3, half of the offspring fed the phyto-600 diets continued to receive that diet and the other half were given the phytoestrogen-free diet for 40 days, beginning at 80 days of age. Both groups were killed at 120 days of age for measurement of the volume of the anteroventral periventricular nucleus ( $\mathrm{n}=5-7 / \mathrm{sex} /$ group). Males and females fed phyto-600 diets throughout their lifetimes displayed the typical pattern of $\sim 2$-fold higher anteroventral periventricular nucleus volume in females compared to males. Males switched to the phytoestrogen-free diet displayed a significant increase (nearly 2-fold) in anteroventral periventricular nucleus volume compared to males who remained on the phyto-600 diet. No effect of the change in diet on anteroventral periventricular nucleus volume in females was detected.

The study authors concluded that phytoestrogens decrease body and prostate weight and affect anteroventral periventricular nucleus volumes in adult males but have no effect on brain aromatase levels.

Strengths/Weakness: A strength of the study was that brain concentrations of daidzein, genistein, and equol were measured in the medial basal hypothalamus and preoptic area, thus verifying that these phytoestrogens reached the hypothalamus. Animals were exposed to phytoestrogens in the diet, which is a relevant route of exposure. Diets were analyzed by HPLC to determine concentrations of phytoestrogens, genistein, and daidzein (although this determination seems to have been made in previous studies). Validation of the time-resolved fluoroimmunoassay method used to measure brain concentrations of phytoestrogens has been published previously. Ovarian tissues were used as a positive control in the aromatase assay. The anteroventral periventricular nucleus sections were traced by two researchers blind to treatment group, and these traced areas were averaged. A weakness is that this study included only one dietary level of soy, which does not allow for evaluation of dose-response relationships. The stability of phytoestrogen-containing diets was not reported. The authors do not provide any litter parameters (e.g., fertility, litter sizes, sex ratio, pup body weights). They also did not provide any body weights at the time of randomization, during gestation and lactation, or for pups at weaning. It is difficult to discern whether the $17 \%$ decrease in body weight in the 75-day-old males maintained on the phytoestrogen-containing diet may have contributed to the alterations in prostate weight. Chapin et al. (1993) reported a significant (19\%) decrease in absolute prostate weights in feed-restricted male rats with a 12\% change in body weight. Thus, prostate weight can be influenced by body weight. It is not clear whether the authors controlled for litter effects (i.e., consideration of the litter as the unit of analysis for animals with lifelong phytoestrogen exposures; fetuses within the same litter exposed to phytoestrogen-containing or phytoestrogen-free diet in utero are not independent samples). Equol antiserum showed some cross-reactivity with dihydrogenistein and dihydrodaidzein. There was no phytoestrogen-free control included in the experiment measuring anteroventral periventricular nucleus volume. The experiment compared rats maintained on a phytoestrogencontaining diet with those switched to a phytoestrogen-free diet at 80 days of age. It would have been useful to determine whether the early, in utero/prenatal/prepubertal exposures to phytoestrogens contributed to long-term differences in anteroventral periventricular nucleus 
area. Protein sources for these diets (Lund et al., 2001a) were soy for the phyto-600 diet and fish meal for the phytoestrogen-free diet. The protein sources likely contributed other differences aside from phytoestrogen levels. Odum et al. (2001) reported that different rodent diets with different amounts of phytoestrogens had centrally mediated effects on rodent sexual development due to nutritional differences between the diets rather than ER-mediated effects. The authors do not discuss how these data relate to human hypothalamic development.

Utility (Adequacy) for CERHR Evaluative Process: This study is not useful in the CERHR evaluation process.

Weber et al. (2001a), supported by the National Science Foundation, examined the effects of low- and high-phytoestrogen diets on aromatase activity on medial basal hypothalamic and preoptic areas of maternal, fetal, and neonatal rats. Sprague-Dawley rats were placed on a high-phytoestrogen $(n=10)$ or a low-phytoestrogen $(n=13)$ diet. The commercially available high-phytoestrogen diet contained phytoestrogens $603 \mu \mathrm{g} / \mathrm{g}$ consisting mainly of daidzin, genistein, and glycitein. According to Lephart et al. (2002), the diet was soy-based. The isoflavone levels were below the detection limits in the low-phytoestrogen diet, which had the same levels of protein, carbohydrates, and fats as the high-phytoestrogen diet. Rats were mated at 80 days of age and remained on their respective diets throughout pregnancy and lactation. At GD 16.5 and 20.5 [day of plug not specified] and on PND 3.5 [day of birth not specified], brains were collected from dams and offspring. At those same time points, offspring ( $\mathrm{n}=16-34 / \mathrm{sex} / \mathrm{group} /$ time period) were sexed, weighed, and evaluated for anogenital distance. The medial basal hypothalamic-preoptic brain area of dams $(n=3-5 /$ time period $)$ and offspring ( $\mathrm{n}=9-15 \mathrm{sex} / \mathrm{group} /$ time period) was incubated in medium with testosterone for determination of aromatase activity by a "tritiated water" method. Isoflavone levels were measured in blood of dams and offspring at each time point, and the results of the analyses are discussed in Section 2.1.2. Data were analyzed by ANOVA, followed by pair-wise comparison with the NewmanKeuls test.

Fetal body weights were significantly higher in the low-phytoestrogen group on PND 20.5; no offspring body weight effects were detected at the other time periods. Anogenital distance was significantly higher in low-phytoestrogen males on GD 20.5, but when normalized for body weight, anogenital distance was significantly greater in high-phytoestrogen males and females. On PND 3.5, anogenital distance was significantly longer in high-phytoestrogen females, and no statistically significant effects were observed when normalized for body weight.

Phytoestrogens crossed the placenta and were lactationally transferred to pups; however, no effects of the phytoestrogen diets on brain aromatase levels in pregnant dams, fetuses, or neonates were detected. The study authors concluded that dietary estrogens did not alter aromatase activity in the medial basal hypothalamic-preoptic brain area during perinatal development.

Strengths/Weakness: A strength of this study was that plasma phytoestrogen levels were measured in pregnant dams (GD 16.5 and 20.5), fetuses (GD 20.5), and neonates (PND 3.5). Animals were exposed to phytoestrogens in the diet, which is a relevant route of exposure. Diets were analyzed by HPLC to determine concentrations of the phytoestrogens genistein, daidzein and glycitein (although this determination seems to have been made in previous studies). Sex, body weight, and anogenital distance were recorded in the offspring. Analysis of blood samples included internal controls to validate the assay and verify recovery. A coefficient of variation was provided to establish assay precision and, as a comparison, adult male plasma phytoestrogen levels were determined for males on the same diets. A weakness of the study is that it included only one dietary level of soy, which did not allow for evaluation of dose-response relationships. The stability of phytoestrogen-containing diets was not reported. It was not specified whether the day on which the females were sperm-positive was 
GD 0 or GD 1, nor was the day of delivery specified as either PND 0 or PND 1. The authors did not provide litter parameters (e.g., fertility, litter sizes, sex ratio). They also did not provide body weights at the time of randomization. It did not seem that authors controlled for litter effects (i.e., in Table 2 and Fig. 1B of the study, they did not consider the litter as the unit of analysis for animals exposed during gestation and lactation). Blood samples were pooled by treatment (representing three to five litters per collection); therefore, variance in blood levels could not be determined (Table 1 of the study). Maternal body weights and body weight gains during gestation were not given. It is puzzling that the increases in relative anogenital distance (normalized to body weight) were transient (not different at PND 3.5). The differences in protein sources (soybeans and fish) likely contributed other differences aside from phytoestrogen levels. Odum et al. (2001) reported that different rodent diets with different amounts of phytoestrogens had centrally mediated effects on rodent sexual development due to nutritional differences between the diets rather than ER-mediated effects. The authors assessed plasma phytoestrogen concentrations by ANOVA (Table 1 of the study), despite using pooled samples and not presenting variances. The authors did not discuss how these data relate to human hypothalamic development.

Utility (Adequacy) for CERHR Evaluative Process: This study is not useful in the CERHR evaluation process.

Lund and Lephart (2001), supported by the National Science Foundation and Brigham Young University, examined the anxiolytic effects of a phytoestrogen-containing diet on rats. Long Evans male and female rats were fed a diet containing phytoestrogens $600 \mu \mathrm{g} / \mathrm{g}$ diet (phyto-600) or a phytoestrogen-free diet. According to Lund et al. (2001a), the phyto-600 diet contained soy and the phytoestrogen-free diet contained fish meal. Diets were similar in protein, fat, amino acid, mineral, and vitamin content. Rats were fed the diets before mating and throughout pregnancy and lactation. On weaning at 30 days of age, 10-14 $\mathrm{F}_{1}$ offspring/sex/group received the same diet as their dams. [The number of litters from which the offspring were obtained was not stated.] Body weight and feed and water intake were measured in offspring at 50 days of age. At 75 days of age, anxiety was assessed in the offspring using an elevated plus-maze. Data were analyzed with ANOVA and Bonferroni post-hoc comparisons. Body weights were significantly lower in males [4\%] and females [5\%] fed the phyto-600 diet, despite a significant increase in feed intake in the phyto-600 males. Water intake was significantly higher in males and females fed the phyto-600 diet. A total of 10-14 rats/sex/group completed the maze test in all groups except the phyto-600 females. Only seven females of the phyto- 600 group completed the test, because five animals fell or jumped off the open arms. The phyto- 600 diet was observed to have anxiolytic effects, as noted by an increased percentage of time spent in open arms [ 60 compared to $30 \%$ in the phytoestrogen-free group] and increased numbers of entries into open arms [ $\sim 45$ compared to $25 \%$ of entries] by both males and females. The study authors concluded that phytoestrogens in soy caused marked anxiolytic effects in rats.

Strengths/Weakness: A strength of this study was that the authors used the dietary route of exposure, which is a relevant route, and the concentration of phytoestrogens was relevant to laboratory animals (commercially available laboratory diet with high phytoestrogen levels; $600 \mu \mathrm{g} / \mathrm{g} \mathrm{diet}$ ). Rats were exposed to phyto- 600 or phytoestrogen-free diets through critical windows of development (pre-mating, mating, gestation, lactation, and into adulthood) to monitor the long-term effect of these different diets. Circulating levels of phytoestrogens using this dosing paradigm were reported in previous publications by these authors. Statistical analyses were appropriate. A weakness is that this study used a single dose level of phytoestrogens (phyto-600), which does not allow for an evaluation of dose-response relationships. It is not clear whether the authors controlled for litter effects (i.e., consideration of the litter of origin when assigning pups to different endpoints or use of the litter as the unit 
for statistical analysis). Fetuses within the same litter exposed to phytoestrogen in utero are not independent samples. Body weights and feed and water consumption were monitored for only 3 days at 50 days of age. Body weights at PND 75, the time of maze testing, were not reported. Rats were tested only once for $5 \mathrm{~min}$ in the plus-maze.

Utility (Adequacy) for CERHR Evaluative Process: This study is not useful in the CERHR evaluation process.

Lund et al. (2001b), supported by the National Science Foundation and Brigham Young University, examined the effects of lifelong phytoestrogen exposure on visual spatial memory in rats. In the first two parts of the experiment, Long-Evans rats were fed either a soy-based diet containing phytoestrogens $600 \mu \mathrm{g} / \mathrm{g}$ diet (phyto-600) or a phytoestrogen-free diet during pregnancy and the lactation period. [The number of dams treated was not specified.] According to Lund et al. (2001a) the protein source for the phytoestrogen-free diet was fish meal. Diets were similar in protein, fat, amino acid, mineral, and vitamin content. Ingestion of the phyto-600 diet was said to result in blood phytoestrogen levels similar to Asians consuming high amounts of soy. The phytoestrogen-free diet was representative of Western populations that consume very little soy. Offspring were weaned 30 days after birth and given the same diets as their mothers. The diet was given ad lib until 50 days of age when feed was limited to maintain the rats at $\sim 90 \%$ of normal body weight. Beginning at 57 days of age, the rats $(n=$ 13-16/sex/group) were tested for their ability to retrieve a food reward from an 8-arm maze. Once the rats mastered the task they were tested for 10 days ( 1 trial/day). After completion of the test, rats were killed and brains were collected for measurement of calbindin and cyclooxygenase- 2 levels by Western blot. The first part of the experiment examined males, and the second part examined females. The two parts of the experiment were conducted independently of one another but at the same time intervals, using the same procedures. Data were analyzed by one-way ANOVA and multivariate ANOVA.

Males fed the phyto-600 diet required significantly more trials to master the 8-arm maze than males fed the phytoestrogen-free diet (13 compared to 11 trials). In contrast, females fed the phyto-600 diet mastered the maze in significantly fewer trials compared to females fed the phytoestrogen-free diet (11.5 compared to 13.5 trials). No effect of diet on working memory was detected [data not shown]. In the frontal cortex, high levels of phytoestrogens corresponded with decreases in calbindin and increases in cyclooxygenase- 2 in males only. Calbindin-protein expression in the frontal cortex but not hippocampus was significantly lower in males fed the phyto- 600 compared to the phytoestrogen-free diet. Cyclooxygenase- 2 protein expression was significantly lower in the frontal cortex of males fed the phytoestrogen-free compared to the phyto- 600 diet. No effects of diet on calbindin and cyclooxygenase-2 protein expression in brain were detected in female rats. The study authors stated that calbindin protects against neurodegenerative diseases and apoptosis through regulation of intraneuronal calcium. Cyclooxygenase- 2 is an inflammatory protein associated with key steps in the development of Alzheimer's disease.

In the third part of the experiment, male and female rats were exposed to the phyto-600 diet from gestation through adulthood, and the 8-arm maze testing (described above) was conducted in 11-13 rats/sex/group, as described for the first and second parts of the experiment. One trial/ day was conducted over a 10-day period. After completion of the maze testing, half the rats were randomly selected to receive the phytoestrogen-free diet and the other half continued to receive the phyto- 600 diet ad lib for 15 days. For the next 10 days, feed was restricted to maintain the rats at $\sim 90 \%$ of normal body weight. When rats were reduced to $90 \%$ of normal body weight, they were tested for 15 days ( 1 trial/day) using an 8 -arm maze in which only four arms contained a food reward ( $\mathrm{n}=5-7 \mathrm{rats} / \mathrm{sex} /$ group). 
Males fed the phyto-600 diet mastered the maze in significantly fewer trials compared to females fed the same diet ( 8 trials in males compared to 12 trials in females). When trial results were averaged over 2-day periods, males had significantly more correct arm entries than females in three of five periods. Significant dietrelated and sex-related effects were observed in the 4-arm maze test that was conducted after half the animals continued to receive the phyto-600 feed and the other half were switched to the phytoestrogen-free feed. Data were presented as the number of correct arm entries and were averaged over a 3-day period. In general, females maintained on the phyto-600 diet performed better than females switched to the phytoestrogen-free diet. In contrast, performance was better in males switched to the phytoestrogen-free diet compared to males that continued to receive the phyto-600 diet. In rats switched to the phytoestrogen-free diets, males performed better than females. During the last two sets of three trials, males switched to the phytoestrogen-free diet made significantly fewer reference errors (entering an unbaited arm) than males maintained on the phyto-600 diet. There was no effect on working memory (re-entering a baited arm) or working/reference (re-entering an unbaited arm). Both sexes maintained on the phyto- 600 diets completed the task in significantly less time than animals switched to the phytoestrogen-free diet.

Isoflavones were detected in several brain regions of $F_{0}$ males fed the phyto- 600 diet in adulthood. Concentrations in frontal cortex were about two orders of magnitude higher than in hippocampus. According to study authors, both of those brain regions are critical for visual spatial memory. Additional details about brain levels of phytoestrogens are provided in the toxicokinetics discussion in Section 2.

The study authors concluded that dietary phytoestrogens caused a reversal in sexual dimorphic expression of visual spatial memory.

Strengths/Weakness: This study had several strengths and was well designed in that the authors exposed rats to phyto-600 or phytoestrogen-free diets through critical windows of development (pre-mating, mating, gestation, lactation, and into adulthood) to monitor the longterm effect of these different diets. Furthermore, by switching some adults to the alternate diet, the authors could examine the reversibility of diet-related differences in maze performance. Diet is a relevant route of exposure, and the dietary concentration of phytoestrogens was relevant to both laboratory animals (commercially available laboratory diet with high phytoestrogen levels; $600 \mu \mathrm{g} / \mathrm{g}$ diet) and humans (reported plasma levels in rats were similar to Asians consuming large amounts of soy protein per day). Maze testing and analysis were conducted blind to treatment group. Measurements were made to determine the levels of phytoestrogens in different brain regions, calbindin in the frontal cortex and hippocampus, and cyclooxygenase- 2 in the frontal cortex. Circulating levels of phytoestrogens using this dosing paradigm were reported in previous publications by these authors. A weakness of this study was that it used a single dose level of phytoestrogens (phyto-600), which does not allow for an evaluation of dose-response relationships. It was not clear whether the authors controlled for litter effects (i.e., that they considered the litter of origin when assigning pups to different endpoints or used the litter as the unit for statistical analysis). Fetuses within the same litter exposed to phytoestrogen in utero are not independent samples. Body weight data were not presented in the manuscript, although the authors noted that body weight effects were the same for males and females and should not have accounted for sexually dimorphic differences in maze performance. In the Methods section, the authors stated that the phyto-600 diet contained phytoestrogens $600 \mu \mathrm{g} / \mathrm{g}$ diet, whereas in the legend to Table 1, it was stated that pups were exposed to phytoestrogens $300 \mu \mathrm{g} / \mathrm{g}$ diet from conception until 50 days of age. The reason for this difference was unclear. The authors also mentioned that plasma levels of phytoestrogens were determined but did not present these data. Brain levels of phytoestrogens were only measured in males. Phyto-600 diet caused opposing effects on visual spatial memory in males and females, yet in examining the frontal cortex, no dietrelated differences in calbindin or 
cyclooxygenase- 2 were detected in females. It would have been useful to measure phytoestrogen levels in brain regions in females to determine if phytoestrogen levels were similar to levels seen in males. The diet change in adulthood from phyto-600 to phytoestrogenfree diet showed reversal of effects (male performance improved, whereas female performance declined); however, the authors did not state whether a complete reversal was achieved. This point is important because a complete reversal of effects would suggest that in utero/lactational exposure did not cause long-term effects on maze performance. Lastly, the authors did not show the data for working memory, which was not altered by diet in either males or females.

Utility (Adequacy) for CERHR Evaluative Process: This study is not useful in the CERHR evaluation process.

Golub et al. (2005), supported by the Violence Research Foundation, evaluated neurobehavioral effects of soy formula in rhesus monkeys. The authors explored the hypothesis that manganese content of formulas would lead to neurobehavioral differences, noting that soy formula has a greater manganese content than does cow-milk formula. Infant monkeys were transferred on the day of birth to a primate nursery and assigned to one of three feeding groups. [Randomization was not specified, but no feeding group differences in dam characteristics or infant birth weights were detected.] A cow-milk formula group was fed Similac ${ }^{\circledR}$ with Iron, which contains manganese $50 \mu \mathrm{g} / \mathrm{L}$. A soy-formula group was fed Baby Basics (a private-label soy formula available at Albertson's), which contains manganese 300 $\mu \mathrm{g} / \mathrm{L}$. A third group was given the soy formula with manganese added to give a concentration of $1000 \mu \mathrm{g} / \mathrm{L}$. Infants were hand-fed for 2 weeks and then progressed to a sipper bottle. At 4 months of age, the monkeys were transitioned to a primate lab diet with supplemental grains, vegetables, and fruit. Behavioral observations and formal neurobehavioral testing occurred over the first 18 months of life. Cerebrospinal fluid was sampled under anesthesia at 4, 10, and 12 months of age and analyzed for homovanillic acid and 5-hydroxyindoleacetic acid.

Statistical analyses were carried out using ANOVA with post-hoc Fisher test. When there was an apparent effect of soy formula, regression analysis was carried out across the three formula groups to evaluate the manganese dose-relatedness of the effect. Manganese dose was calculated based on formula intake during the first 2 weeks of life.

No feeding group effects on body weight or length were detected during the first 4 months of life, when the formulas were being given. Between 6 and 9 months of age, the groups that received soy formula lagged "slightly" in height and weight [data not shown; individuals not weighed or measured after 9 months]. No significant feeding group effects on cerebrospinal fluid levels of homovanillic acid or 5-hydroxyindoleacetic acid were detected. Soy-fed infants initiated more behaviors than did cow milk-fed infants. Wake periods were shorter and sleep periods longer in soy formula-fed compared to cow milk formula-fed monkeys at 8 months of age but not at 4 months of age. Initiation and participation in play activity was decreased among soy formula-fed monkeys compared to cow milk formula-fed monkeys. This effect showed a significant correlation with manganese intake in the first 2 weeks of life. Behavioral response to apomorphine injection was also correlated with manganese intake. Temperament ratings and frequency of stereotypy were not affected by feeding group status. Infants in the soy formula-fed group were described as participating less readily in the structured testing, and the authors suggested that testing at an older age would be useful.

The authors concluded that integration of the behavioral findings was difficult but that increased behavioral changes, altered diurnal rhythms, and reduced play behavior may indicate altered regulatory control.

Strengths/Weaknesses: A strength of the study is that the dosing scenario is directly relevant to human exposures. Endpoints were evaluated from birth to 18 months of age to determine 
longer-term effects of neonatal exposure. The experimental model was the rhesus monkey, which is similar to humans in brain maturation at the time of birth. Infant monkeys were reared under standardized conditions (not specified). To assess a variety of behaviors, the authors selected a pre-determined number of sessions during which data for different endpoints were collected. Not all infant monkeys could generate useful data during the limited test sessions, so participation criteria were established for each test to identify data for exclusion. Observer reliability for scoring motor and postural behavior was established before the experiments. For social observations, infants were paired with a different infant for each session (one with whom they had not interacted socially) and monitored by a familiar observer. To alleviate interobserver variability within a test, one observer collected all social dyad observations and another recorded all stereotypy observations. For the cognitive testing, performance criteria were established. Once these criteria were exceeded, a switch or reversal of the reward was introduced. A weakness of this study was that it used a single dose level of soy formula, which does not allow for an evaluation of dose-response relationships for soy formula (although the dose-response relationship for manganese could be evaluated). There was no information provided as to how infants were randomized into the various treatment groups (although pregnancy housing conditions were controlled across treatment groups). The authors stated that body weight and lengths were affected in the soy-fed animals between 6 and 9 months of age, but these data were not shown. Apparently, individual weights were not collected after 9 months of age (monkeys were weighed as a group). Cow-milk and soy formula differ in many other factors aside from soy, including nutritional components related to protein, carbohydrate, and fat. Sample sizes were relatively small for some neurobehavioral endpoints ( $\mathrm{n}=8$ /group, although this sample size is good for a primate study). At 4 months of age, the primates were transitioned to commercial nonhuman primate diet with enrichment foods. It is not known whether these dietary components contributed additional phytoestrogen exposures. Because social observations were discontinued at different times, the largest number of sessions completed by all monkeys was used for analysis; thus, some data were discarded. Cerebrospinal fluid metabolites and social interaction endpoints were not highly correlated. Infants carried out the delayed nonmatch to sample task at chance level, indicating that the young monkeys were unable to learn this task.

Utility (Adequacy) for CERHR Evaluation Process: This study is not useful in the CERHR evaluation process.

\subsubsection{Other endpoints}

3.2.4.1 Rat: Chang and Doerge (2000), of the FDA, examined the effects of post-weaning and adult exposure to soy diets on thyroid function in rats. The effects of genistein added to diet were also examined, and that portion of the study is discussed in the CERHR Expert Panel Report on Genistein (Rozman et al., in press). On PND 21-190, two groups of male and female Sprague-Dawley rats were fed either NIH 31, a soy-based diet reported to contain genistein and daidzein at $30 \mathrm{ppm}$ each or a soy-free $5 \mathrm{~K} 96$ feed. [The number of rats treated was listed as six, and it is assumed that it was 6/sex/dose as in the main part of the study, which looked at genistein effects and is discussed in the CERHR Expert Panel Report on Genistein (Rozman et al., in press).] Microsomal thyroid peroxidase activity was determined using a spectrophotometric method to measure guaiacol oxidation. [It is assumed that data were analyzed using 2-way ANOVA or Dunnett test as in the main part of this study.]

Thyroid peroxidase activity was significantly reduced in rats fed the soy-based compared to soy-free diet. Thyroid peroxidase activity in the soy diet group was $<50 \%$ the activity in the soy-free diet group. The study authors noted that the effect was similar to that observed in rats fed diets containing $100 \mathrm{ppm}$ genistein in the aglycone form, as discussed in greater detail in the CERHR Expert Panel Report on Genistein (Rozman et al., in press). Thus, it was noted 
that the form of genistein did not affect total serum isoflavone concentrations or decrease thyroid peroxidase activity inhibition. Serum levels of genistein and daidzein were also measured and are discussed in Section 2. The study authors noted that consumption of isoflavones by humans could result in uptake by thyroid gland and inactivation of thyroid peroxidase.

Strengths/Weakness: A strength of this study was the measurement of total genistein and daidzein levels in serum using HPLC with electrospray MS, a method that generated very low detection levels ( $20 \mathrm{nM}$ genistein in serum). Thyroid peroxidase assay procedures were validated for linearity of response versus protein, and steps to prevent loss of thyroid peroxidase activity during isolation were well described. Assuming that statistical analyses were conducted as in the main part of the study examining addition of genistein to feed, the analyses were appropriate, using two-way ANOVA to examine dose, sex, and dose/sex interactions. A weakness of this study is that stability of genistein in the diet was not verified. Isoflavone dose levels were not reported in $\mathrm{mg} / \mathrm{kg}$ bw/day, and feed consumption data were not provided. There were no details as to how prepubertal pups were assigned to treatment groups and no indication that the authors controlled for litter effects. There is no indication that the authors controlled for necropsy stress when thyroid hormone samples were collected. Stress can impact the levels of thyroid hormones measured and increase variance (Döhler et al., 1979). It is not clear why rats were killed at 190 days in the diet-comparison study compared to 140 days in the genisteinsupplemented diet study. The authors did not include a discussion on the sensitivity of the rat model to thyroid perturbations (e.g., McClain, 1995).

Utility (Adequacy) for CERHR Evaluative Process: This study is not useful in the CERHR evaluation process.

Teichberg et al. (1990, 1992), supported by the Public Health Service, evaluated the effects of early weaning to artificial diets on gastrointestinal maturation in rats. The marker of gastrointestinal maturation was the development of a barrier to the absorption of horseradish peroxidase, a 40-kD macromolecule. In the normal rat neonate, macromolecular absorption occurs until PND 17, after which absorption decreases and becomes blocked (referred to as closure) by PND 21. In these experiments, Wistar rat pups were distributed among dams to a density of 12 pups (males and females) per dam. On PND 17, the pups were divided into three groups of four pups each. One group stayed with the dam and was nursed, and two groups were given formula as their nutrition source using standard water bottles. The formulas included a protein-hydrolysate formula (Nutramigen ${ }^{\circledR}$ ) and a soy formula (Isomil®). Formula group assignments were balanced for sex and weight. In the first study (Teichberg et al., 1990), a separate weight-matched control group was constituted of pups that remained with their dams in litters of 12 until PND 21. On PND 21, "several litters" of pups were anesthetized and 15 $\mathrm{cm}$ loops of jejunum with intact vasculature were isolated. A solution containing horse-radish peroxidase was placed into the isolated loop and left for $30 \mathrm{~min}$. The luminal fluid was then collected and evaluated for volume. Horseradish peroxidase concentration was measured in blood to assess absorption. Intestinal loops were processed for histochemical localization of horseradish peroxidase using phase-contrast and electron microscopy. To examine the role of glucocorticoids, the first study (Teichberg et al., 1990) administered intraperitoneal (i.p.) hydrocortisone on PND 14 to some of the pups, and the second study (Teichberg et al., 1992) included corticosterone at two different concentrations in the formulas of some of the pups. The use of the glucocorticoid was based on the observation that glucocorticoids advanced intestinal closure in rats.

Both artificial diets in both studies were associated with about a $10 \%$ decrease in mean offspring weight on PND 21; however, no difference was detected in weights of the pups in the first study (Teichberg et al., 1990) if control pups were left in litters of 12 through PND 21. Pups 
fed either formula showed an increase in horse-radish peroxidase absorption on PND 21 compared to pups that continued to be nursed by their dams. Histochemical evaluation of jejunal sections showed horseradish peroxidase confined to the brush border of the mucosa in control animals, whereas in animals given formula, horseradish peroxidase was evident within absorptive epithelial cells. The number of horseradish peroxidase-positive cells was greater in the soy formula-fed rats than the protein hydrolysate-fed rats. In the second study (Teichberg et al., 1992), extension of the artificial feeding period to PND 26 showed intestinal closure to horseradish peroxidase absorption on PND 26 equivalent to that of control animals on PND 21 , suggesting that the artificial diets delayed but did not prevent closure. In both studies, glucocorticoid treatment prevented the delayed closure associated with artificial diets. The authors could not identify the mechanism by which glucocorticoids prevented the delayed closure associated with artificial diets. They believed that soy produced inflammatory epithelial damage, associated with eosinophil infiltration of the lamina propria, but found that low-dose corticosterone, equivalent to concentrations normally present in rat milk, prevented the delay in soy-associated intestinal closure without preventing eosinophil infiltration. They also noted that the effect of protein-hydrolysate formula in delaying closure was similar to that of soy and did not seem to be associated with inflammatory damage to the intestine. The authors pointed out that intestinal absorption of macromolecules is quantitatively less important in the human neonate than in the rat, and that intestinal closure is a less dramatic event in humans.

Strengths/Weaknesses: A strength of this study is that rat pups were fed soy formula, a relevant dosing scenario. The authors tried to balance sex and weight among the treatment groups when assigning rat pups. These studies were conducted on the last 4 days before weaning, a critical time for small intestine epithelial closure and a period when pups are sufficiently mature to feed independently and regulate body temperature. A weight-matched control group was included to account for the decreased body weights in the pups fed protein-hydrosylate and soy formula. The authors reported the endogenous peroxidase activity in pups not given horseradish peroxidase. Serum albumin levels were included as an assessment of nutritional status of the pups. The numbers of horseradish-positive crypt cells were assessed blind to treatment group. The low dose of corticosterone was equivalent to corticosterone levels in the maternal milk of unstressed lactating rats, whereas the higher dose was included for doseresponse purposes. RIA was used to verify the absence of detectable levels of corticosterone in protein-hydrosylate and soy formulas in Study 2. Intestinal tissue was examined in pups from greater than or equal to three experiments carried out at different times. These examinations were conducted blind to treatment group. Controls included intestinal loops from animals not exposed to horseradish peroxidase. For these experiments, sample sizes seemed to have been sufficient, although there was no indication that the authors controlled for litter effects. A weakness is that both studies used only one dose level of soy formula, which does not allow for evaluation of dose-response relationships. Data were generated using the formula Isomil@, and it is difficult to determine whether these data apply to all soy infant formulas. There was no evidence that the authors controlled for litter effects or that that the litter was used as the unit of analysis. The number of pregnant dams from which the litters originated was not specified. Aside from maternal milk, removing pups from the lactating dam on PND 17 likely introduced other stress factors, which may have contributed to study outcome. The difference in horseradish absorption for pups given soy formula and soy

formula1 hydrocortisone was not statistically significant. Rat pups and human infants may vary in some aspects of gastrointestinal tract maturation; the authors state that human infants have immunoglobulin absorption across the placenta, which complicates studies on neonatal intestinal absorption. The significance of these data for humans is unclear, as intestinal absorption of macromolecules is less significant in humans. Controls not exposed to horseradish peroxidase showed peroxidase activity in eosinophils, neutrophils, monocytes, and erythrocytes. 
Utility/Adequacy for CERHR Evaluation Process: This study is not useful in the CERHR evaluation process.

Churella et al. (1976), from Ross Laboratories, examined the effect of trypsin-inhibitor levels in soy formula on development of rats. Rat diets containing varying levels of trypsin inhibitors were prepared by using soy formulas that were not treated, were heat processed, or were heat processed and sterilized to reduce trypsin-inhibitor activity. At 21 days of age, 12 SpragueDawley rats/group were given feed containing $235,40,10.4,14.7$, or 11.5 trypsin-inhibitor U/ $100 \mathrm{~g}$ feed for 3 weeks. A control group was fed a casein-based diet containing 1.5 trypsininhibitor units. Diets were fairly similar in protein, fat, carbohydrate, and $d, l$-methionine content. Food intake and body weights were measured over the 3-week period. Animals were then killed for measurement of final body weights and liver and pancreas weights. Pancreases were examined histologically. [Histologic methods were not discussed, and it was not stated how many rats per group were examined.] Data were evaluated by ANCOVA. Compared to controls, liver weights relative to body weights were significantly reduced [by $\sim \mathbf{1 0 \%}$ ] in rats fed diets with 40 or 11.5 trypsin-inhibitor units. [Absolute organ weights were not reported.] No effects were detected on body weight gain, relative pancreas weight, or pancreatic hypertrophy or hyperplasia. In the analysis of five infant soy formulas, four were found to have trypsin-inhibitor activity at $<15 \%$ of the activity of soy-protein isolate used to manufacture the formulas. The study authors concluded that residual trypsin-inhibition activity is low in soy formulas and, at those levels, would be of no nutritional significance to the rat.

Strengths/Weaknesses: A strength of this study is that diets included soy-based infant formulas (at different stages of processing to heat-inactivate soybean trypsin inhibitor, soy isolate (the soy protein used to manufacture formulas), and a casein-based rodent diet. Duration of the treatment period ( 3 weeks) was determined in a probe study, which showed this as the time of maximum pancreatic hypertrophy for rats fed unheated soy protein. Weanling rats were placed into treatment groups such that each group had similar body weight distributions. The authors calibrated the trypsin-inhibition assay, demonstrating that $1 \mathrm{~g}$ purified soybean-trypsin inhibitor inhibited $1.64 \mathrm{~g}$ trypsin. The impact of processing soy isolate on its soybean-trypsin inhibitor content was evaluated. In deriving the soybean-trypsin inhibitor inhibition curve, extract concentrations were used in a range that inhibited 20-80\% of trypsin activity, as values outside this range were not linear. A weakness of this study is that there was no indication that the authors controlled for litter effects. Although these experiments are helpful for examining soybean trypsin-inhibitor activity, it is difficult to judge the effects of the different formulas as the authors supplemented the lyophilized formulas with numerous other components (carbohydrate, fat, minerals, vitamins, and methionine) when preparing the diets. The trypsin used to analyze soybean trypsin-inhibitor activity was 52\% pure. Dose-response could only be evaluated for soybean trypsin-inhibitor activity. Limited endpoints were examined in the study. Histology procedures were not described. Weanling rats were used in this study, so critical developmental stages during the neonatal period were not evaluated.

Utility/Adequacy for CERHR Evaluation Process: This study is not useful in the CERHR evaluation process.

Ronis et al. (1999), supported by the USDA, evaluated the expression of CYP3A and CYP2B enzymes in male Sprague-Dawley rats exposed to soy protein isolate during development. Parental rats were placed on AIN 93G diet in which soybean oil was replaced by corn oil. The protein source in the diet was casein for one group and soy protein isolate for the other group of parental animals. After several weeks on the diets, animals were mated and females were maintained on their assigned diets through pregnancy and lactation. Litters were adjusted at birth to five males and five females. Male pups were weaned to their dam's diet. Genistein consumption [age unspecified] was estimated at $19.3 \mathrm{mg} / \mathrm{kg}$ bw/day. On PND 65, males from 
each diet group received either dexamethasone or corn oil vehicle by gavage for enzyme induction. Livers were removed the next morning and microsomes were prepared using differential ultracentrifugation. Assays of CPY3A activity were carried out for erythromycin $N$-demethylase, ethylmorphine $N$-demethylase, testosterone $6 \beta$-hydroxylase, lithocholic acid $6 \beta$-hydroxylase, and corticosterone $6 \beta$-hydroxylase. CYP2B was assessed by the activity of pentoxyresorufin $O$-depentylase. Western blotting was used to measure microsomal CYP3A and CPY2B1 apoproteins and Northern blotting was used for analysis of several isozymes of CYP3A as well as CYP2B1 mRNAs. Statistical analysis was carried out using two-way ANOVA followed by Student-Newman-Keuls test. Most comparisons were carried out using rats from four to six litters/group.

Body weight was $12 \%$ lower in animals fed soy protein isolate than animals fed casein. There were no dietrelated differences in absolute or relative liver weight. In rats not receiving dexamethasone, corticosterone $6 \beta$-hydroxylase was increased $9 \%$ by soy protein isolate, but other CYP3A activities were unaffected. In rats receiving dexamethasone, all CYP3A activities except lithocholic acid 6 $\beta$-hydroxylase (CYP3A18) were increased by soy protein isolate. Pentoxyresorufin $O$-depentylase was increased by soy protein isolate in rats induced with dexamethasone. Western blotting showed an increase in CYP3A apoprotein in soy protein isolate-fed rats that received dexamethasone, and Northern blotting showed an increase in mRNA for $C Y P 3 A 2$ in these animals. No effects of diet on $C Y P 2 B 1 \mathrm{mRNA}$ and apoprotein were detected. The authors concluded that soy protein isolate increased expression of CYP3A2, particularly in dexamethasone-induced animals. They postulated that some of the variability in human neonatal hepatic CYP3A enzyme activity may be related to dietary intake of soy infant formula.

Strengths/Weaknesses: Strengths are the use of AIN 93G chow with soybean oil replacing corn oil resulting in a feed with expected low phytoestrogen content, the use of several methods to analyze CYP induction (enzyme activities, mRNA levels, and apoprotein levels), determination of cross reactivity of antibodies for Western blots, and use of the litter as the experimental unit in assays. Weaknesses were the lack of determination of phytoestrogen content of chow, the use of different protein sources in chow, the failure to specify the length of time on diet before breeding and the method of selection of male pups from each litter, the examination of only males, the small number of animals per group, the lack of indication of when/if feed consumption was monitored or whether feed consumption was similar between groups (a difference may have affected body weight, which was decreased in rats consuming soy protein isolate), lack of a loading control for Western blots, and lack of indication how pooling of liver microsomes was accomplished.

Utility (Adequacy) for CERHR Evaluation Process: This study is not useful for the CERHR evaluation process.

Ronis et al. (2004), supported by USDA, evaluated hepatic CYP3A in weanling male SpragueDawley rats exposed during pregnancy and lactation to dietary soy protein isolate. Pregnant dams were obtained on GD 4 and fed AIN 93G diets in which soybean oil was replaced with corn oil. In one group of dams, the dietary protein source was casein and in another group, the dietary protein source was soy protein isolate ( $\mathrm{n}=7-9$ /group). The soy protein isolate diet contained isoflavones at $430 \mathrm{mg} / \mathrm{kg}$ feed (genistein $276 \mathrm{mg} / \mathrm{kg}$ feed and daidzein $132 \mathrm{mg} / \mathrm{kg}$ feed). At birth, litters were adjusted to five males and five females. Male pups were weaned on PND 21 to their dam's diet. On PND 25, male pups were given corn oil vehicle or the CYP3A inducers dexamethasone or clotrimazole by gavage $(\mathrm{n}=1 \mathrm{pup} / \mathrm{treatment/litter})$ or were untreated (two pups/litter). Livers were removed the next morning and microsomes were prepared using differential ultracentrifugation. PND 25 pups in the soy protein isolate group were estimated to have consumed dietary isoflavones at $65 \mathrm{mg} / \mathrm{kg}$ bw/day. Hydroxylation of 
testosterone and midazolam were measured in microsomal preparations as estimates of CYP3A mono-oxygenase activities. CYP3A apoproteins were estimated by Western blot, and CYP3A mRNAs were estimated by Northern blot. CYP3A1 heterologous nuclear RNA was measured using RT-PCR. Statistical analysis was carried out using $t$-tests or two-way ANOVA followed by Student-Newman-Keuls test; most groups contained rats from seven to nine litters. [The Expert Panel notes that legends for several figures indicate that some groups contained only four or five rats.]

Dietary soy protein isolate resulted in the presence of CYP3A apoprotein in hepatic microsomes, whereas casein-fed animals had undetectable CYP3A apoprotein. The soy protein-associated increased levels of CYP3A were further enhanced by both of the CYP3A inducers. CYP3A2 mRNA and hydroxylation of midazolam (particularly 1-hydroxylation) were also increased by soy protein isolate in untreated pups. This difference in midazolam hydroxylation was not observed among pups treated with the corn oil vehicle; however, formation of the 1,4-dihydroxylated product was decreased by consumption of soy protein isolate. No diet-related alteration in CYP3A1 heterologous nuclear RNA was detected. The authors concluded that the increase in CYP3A activity associated with feeding soy protein isolate might result in altered metabolism of medications by infants on soy formula.

Strengths/Weaknesses: This study shares several features and, therefore, strengths and weaknesses, with the previous study (Ronis et al., 1999). In addition, there was no indication of how phytoestrogen content of soy protein isolate was determined (presumably by company that supplied it). Feed consumption presumably was monitored but there was no indication of how often, and isoflavone intake was only estimated for one time point (PND 25). The corn oil vehicle seemed to have an effect, at least on midazolam metabolism.

Utility (Adequacy) for CERHR Evaluation Process: This study is not useful for the CERHR evaluation process.

Ronis et al. (2006), supported by USDA and the Solae Company, evaluated the effect of dietary soy protein isolate during development on hepatic CYP3A1 and CYP3A2 in prepubertal Sprague-Dawley rats. Pregnant dams were obtained on GD 4 and fed AIN 93G diets in which soybean oil was replaced with corn oil. In one group of dams, the dietary protein source was casein and in another group, the dietary protein source was soy protein isolate. At birth, litters were adjusted to five males and five females. Dams continued on their assigned diets until PND 15 at which time five of 11 casein-fed litters were switched to the soy protein isolate diet, and four of seven soy protein isolate-fed litters were switched to the casein diet. Food pellets were added to the cage bottoms to facilitate direct consumption by pups. Rats were weaned to their respective diets on PND 21, and livers were collected from the pups on PND 33. A second experiment was carried out in which casein diets were used in 18 dams/litters from GD 4 until PND 15 at which time six litters were maintained on the casein diet and three litters/group were switched to the soy protein isolate diet, a diet supplemented with ethanol-washed (isoflavonepoor) soy protein isolate, a casein diet supplemented with genistein $250 \mathrm{mg} / \mathrm{kg}$ feed, or a casein diet supplemented with daidzein $250 \mathrm{mg} / \mathrm{kg}$ feed. On PND 33, livers and jejunum were collected. The unwashed soy protein isolate diet was estimated to provide pups a daily isoflavone intake of $65 \mathrm{mg} / \mathrm{kg}$ bw between PND 21 and 33. Hepatic microsomes were prepared by differential ultracentrifugation, and testosterone $6 \beta$-hydroxylase activity was assessed in males. Western immunoblotting was used to measure CYP3A1 and CYP3A2 in hepatic microsomes, and RT-PCR was used to quantify CYP3Al and CYP3A2 mRNA in liver and jejunum. Statistical analysis was carried out using two-way ANOVA followed by StudentNewman-Keuls test. Testosterone $6 \beta$-hydroxylase activity was analyzed using non-parametric tests. 
No increase in pup hepatic CYP3A1 and CYP3A2 mRNA was detected when soy protein isolate exposure was restricted to pregnancy and the first 2 weeks of lactation. Exposure on PND 1533 increased CYP3A1 mRNA in males and females and increased CYP3A2 mRNA in males; exposure to soy protein isolate throughout gestation and to PND 33 increased both CYP3A mRNAs in males and females. Jejunum CPY3A1 mRNA was increased in both sexes by exposure to soy protein isolate on PND 15-33; no alterations in CYP3A2 mRNAs were detected in either sex. In males, hepatic CYP3A1 apoprotein was increased by exposure on PND 1533 to soy protein isolate but not ethanol-washed soy protein isolate, genistein, or daidzein. CYP3A2 apoprotein was increased in males by exposure on PND 15-33 to daidzein but not by exposure to soy protein isolate or genistein. In females, CYP3A1 apoprotein was increased by exposure on PND 15-33 to soy protein isolate but not ethanol-washed soy protein isolate, genistein, or daidzein. Both ethanol-washed and unwashed soy protein isolate increased CYP3A2 apoprotein in females exposed on PND 15-33. Hepatic testosterone 6 $\beta$-hydroxylase activity in males was increased by exposure on PND 15-33 to ethanol-washed or unwashed soy protein isolate or daidzein but not genistein. The authors concluded that developmental changes in CYP3A expression was not due to "imprinting" during pregnancy or early lactation and that soy isoflavones may have different effects on CYP3A induction than other components of soy protein isolate.

Strengths/Weaknesses: Strengths are the use of AIN 93G chow with soybean oil replacing corn oil resulting in a feed with expected low phytoestrogen content, the use of several methods to analyze CYP induction (enzyme activities, mRNA levels, and apoprotein levels), and determination of cross reactivity of antibodies for Western blots. GADPH was used as internal control for RT-PCR quantification of CYP3A mRNAs, but there was no indication that any of the treatments affected $G A D P H$ expression. Additional strengths are the examination of males and females, the cross-over experimental design to determine reversibility of effects, and the examination of effects of genistein and daidzein independently, although inclusion of these compounds in the ethanol-washed soy protein isolate chow would have been preferred. Weaknesses include the lack of determination of phytoestrogen content of the chow, the use of different protein sources, the lack of indication of how phytoestrogen content of soy protein isolate was determined (presumably by company that supplied it), the lack of indication of the purity of genistein and daidzein, failure to consider litter as the experimental unit, and the use of only three litters in several treatment groups. Feed consumption presumably was monitored, but there was no indication of how often and isoflavone intake was only estimated for one time point and one diet (PND 21-33 consuming ethanol-washed soy protein isolate).

Utility (Adequacy) for CERHR Evaluation Process: This study is not useful for the CERHR evaluation process.

3.2.4.2 Pig: Li et al. (1990), funding not indicated, examined the effects of soy diet on hypersensitivity in early-weaned pigs. Four sows were fed corn-gluten meal from GD 109 through lactation. Piglets (Hampshire $\times$ Yorkshire $\times$ Duroc) from those four litters were randomly assigned to a soy or milk (control) group $(n=16$ /group). From 7-14 days of age, piglets were sensitized by oral gavage with $5 \mathrm{~g} /$ day of their respective protein treatment, $48 \%$ soybean meal or dried skim milk (control group). Pigs were weaned at 21 days of age and fed a milk-protein or a soybean-meal diet ad lib until 56 days of age. [Lactose was listed as the carbohydrate source for the soybean diet but not specified for the milk-protein diet. Lactose was assumed to be the carbohydrate source in the milk-protein diet, but the percentage in diet was not specified. Both diets contained soybean oil.] One day before termination, blood was drawn to obtain lymphocytes and measure IgG titers to soy protein. In addition, dermal soybean hypersensitivity was tested. Half the pigs (two per litter) were killed at 28 days of age, and the other half were killed at 56 days of age. Duodenum samples were removed to collect lymphocytes and examine villous height and crypt depth by scanning 
electron and light microscopy. Blastogenic response was determined in lymphocytes. Mitogenic responses were measured in lymphocytes and mononuclear cells. Data were analyzed by ANOVA and Student $t$-test.

Body weight gain was reduced at 28 days of age $(P<0.01)$. No effects of diet on serum concentrations of zinc, selenium, iron, and copper were detected at 28 and 56 days of age. No significant differences in skin thickness after intradermal injection of soy or milk proteins were observed between the soybean- and milk-diet groups at 27 and 55 days of age. However, IgG titers to soy were significantly increased in both age groups of piglets fed soybean compared to milk-protein diets. At 28 days of age, intestinal villi of pigs fed soybean diets were shorter and broader than those of pigs fed milk-protein diets, but this difference was not observed at 56 days of age. At 28 and 56 days of age, intestinal crypt depth was slightly higher in pigs fed soybean diets. Neither diet was observed to have a significant effect on blastogenic responses of peripheral or intestinal lymphocytes collected at either age and cultured with purified soy proteins, phytohemagglutinin, or pokeweed.

The study authors concluded that pigs sensitized to soybean protein and fed a soybean-meal diet displayed transient hypersensitivity that was evidenced by decreased villous height and increased IgG titers. The effects coincided with reduced growth from 3-4 weeks.

Strengths/Weaknesses: A strength of this study is that sows received a corn-corn gluten diet to prevent the passive transmission of soybean-protein antibodies to the offspring. Pigs in each litter were assigned randomly to treatment groups, and it seems that the authors assigned piglets within the same litter to different treatment groups (i.e., four litters were used and at PND 28 necropsy, eight animals were randomly selected [two pigs/litter] from each treatment for necropsy). Pigs received soybean orally, which represents a relevant route of exposure. Triplicate intestinal villi samples (10 villi/specimen) were measured for each pig. Villous atrophy has been reported in other species after inducing hypersensitivity to soy proteins. A weakness of the study is that the authors used only one dose level of soy, so dose-response relationships could not be evaluated. Pigs originated from only four litters. There was no indication that the authors controlled for litter effect during their data analyses. Aside from soybean meal, there were other differences between the two weanling diets. Abnormal villous morphology and increased serum IgG titers corresponded with a period of decreased growth in soy-fed weanlings. As the authors point out, "low preweaning feed intake, which commonly occurs when weaning at $3 \mathrm{wk}$ or less, results in a transient hypersensitivity to dietary antigens." It is interesting that skin-fold thickness was doubled in pigs maintained on a milk protein-based diet and given a milk-protein injection on PND 27, particularly given that milk-protein allergies are common in children. There was no increase in serum anti-soy antibody IgG titers in milk proteinfed pigs, but anti-milk-protein $\mathrm{IgG}$ was not tested. The contribution of early weaning stress to these effects is not known.

Utility/Adequacy for CERHR Evaluation Process: This study is of limited utility in the evaluation process.

Zijlstra et al. (1996), funding not indicated, examined the short-term metabolic effects in neonatal piglets fed soy formula. Two separate experiments were conducted 12 months apart. In the first experiment, a catheter was inserted into the umbilical artery of 1-day-old neonatal pigs. Pigs were fed by gavage dosing with casein-whey protein formula or hydrolyzed-soy formula for $36 \mathrm{hr}$ and then received only water for $12 \mathrm{hr}$. A catheter was inserted into the portal vein of neonatal piglets (large White $\times$ Pig Improvement Company), and baseline blood samples were drawn from the catheters $1 \mathrm{hr}$ later. The piglets were gavaged with formula (20 $\mathrm{mL} / \mathrm{kg}$ bw) containing cow-milk protein (40:60 casein:whey ratio), hydrolyzed-soy protein, or intact-soy protein. Arterial and portal blood samples were collected at 15, 30, 60, and 120 min 
after dosing. The second experiment was similar to the first except that only an arterial catheter was inserted, and blood sampling was extended to $3 \mathrm{hr}$. There was also a difference in formula carbohydrate source. In Experiment 1, carbohydrate sources were glucose polymers in soyprotein formulas and lactose in cow-milk protein formula. In Experiment 2, the soy and cowmilk formulas were standardized to contain equal amounts of reduced lactose and glucose polymers. Soy diets were fortified with $d, l$-methionine and $l$-lysine in both experiments. Parameters measured included plasma amino acid levels using anion-exchange chromatography, insulin and glucagon levels by RIA, and glucose concentrations using a glucose-oxidase method. In Experiment 2, piglets were killed after the last sampling time and gastrointestinal contents were weighed to determine gastric emptying. Each experiment was conducted as four replicates of six littermates. Within each replicate, two piglets were assigned randomly to each of the three treatment groups. Therefore, there were a total of eight piglets in each treatment group in Experiments 1 and 2. [Data were analyzed according to a randomized complete block design, but the statistical tests were not specified.]

In the 48-hr period before formula dosing and blood sampling in both experiments, piglets fed casein-whey formula gained weight, whereas piglets fed hydrolyzed-soy formula lost weight. Diarrhea was observed in 15 of 16 piglets fed the hydrolyzed-soy formula in Experiment 1 and in all piglets fed the casein-whey and hydrolyzed-soy formulas in Experiment 2. The study authors noted that concentrations of glucoamylase and maltase are low in the digestive tracts of piglets. Therefore, there may have been impaired digestion and absorption of the carbohydrates used in soy formulas in Experiment 1 and all formulas in Experiment 2. The authors also cited studies noting that soy proteins were shown to cause small intestine mucosa damage resulting in malabsorption and diarrhea in pigs.

In Experiment 1, differences between amino acid concentrations in portal compared to arterial blood after dosing with all formulas indicated absorption from the gut. In piglets fed caseinwhey formula, the difference between amino acids in portal compared to arterial blood peaked within $15 \mathrm{~min}$, indicating rapid digestion and absorption of proteins. The post-feeding rise in amino acid levels in portal blood was slower and peaked at a lower level with soy formulas, suggesting attenuated digestion and absorption of proteins. Higher concentrations of amino acids in portal compared to arterial blood were maintained for $2 \mathrm{hr}$ after feeding only in the piglets given hydrolyzed soy. In Experiment 2, patterns of amino acid concentrations in arterial blood were similar to those in Experiment 1. Weight of material remaining in stomach and small intestine did not differ between groups, leading study authors to conclude that differences in gastric-emptying rates were not the reason for altered plasma concentrations between the different feeding groups. In Experiment 1, insulin and glucose concentrations were significantly increased at 15-30 min after feeding in arterial and portal blood of piglets fed casein-whey compared to either soy formula. No significant effects on glucagon levels were detected. In contrast to Experiment 1, no significant differences were detected in arterial blood concentrations of insulin, glucagon, or glucose at 15-30 min after feeding in Experiment 2.

The authors concluded that "hydrolyzed soy protein did not result in elevated nutrient concentrations or hormone responses compared with intact soy or [cow] milk protein." Standardization of carbohydrate sources among formulas in Experiment 2 but not Experiment 1 was suggested by study authors as a possible reason for differences in results between the first and second experiments.

Strengths/Weaknesses: A strength is that during the experiments, formulas were stirred constantly to ensure homogeneity, and formulas were replaced every $12 \mathrm{hr}$. Soy hydrolysis was examined using HPLC. The authors partially controlled for litter effect by using two piglets/litter for each treatment group. Treatment groups were equally represented in each replicate. Pigs received formulas orally, which represents a relevant route of exposure. To avoid 
potential effects on small intestine structure and function before sample collection, pigs were maintained on the hydrolyzed soy-protein formula before the metabolic study when both hydrolyzed- and intact-soy protein were used. The authors monitored the amount of dry matter in the stomach and three segments of the small intestine to confirm that differences in absorption were not related to differences in transit time through these gastrointestinal segments. Statistical analyses included a randomized complete-block design to account for different study replicates, although it is not clear that the authors considered the litter in their analysis. A weakness is that the authors used only one dose level of hydrolyzed- and intactsoy protein, so dose-response relationships could not be evaluated. In both experiments, hydrolyzed soy protein-treated pigs lost weight, whereas control pigs fed casein-whey protein gained weight over the monitoring period before formula dosing. In the first experiment, hydrolyzed-soy protein-fed pigs had diarrhea, and both hydrolyzed soy protein-and casein whey-fed groups had diarrhea in Experiment 2. Pigs fed casein-whey protein had greater glucose and insulin concentrations in Experiment 1, which the authors corrected in Experiment 2 by normalizing dietary carbohydrate composition. Each of these factors may have contributed to differences in response. The authors study pooled SEM, so it is not possible to determine the variability around individual means. It is unclear why the authors considered $P<0.05$ to be significant, but noted $P<0.1$ in figures.

Utility/Adequacy for CERHR Evaluation Process: This study is not useful in the CERHR evaluation process.

\subsection{Utility of Data}

3.3.1 Human data-There are a large number of studies describing growth or other health parameters in humans exposed to soy infant formula. Thirty-eight articles were judged to have some utility in the evaluation process; however, the utility of these articles was limited. Limitations encountered commonly included the non-random or unspecified method of assignment to feeding groups, the use of self-selected breast- and formula-feeding mothers, failure to control for the reasons for which soy formula was used, and the early and inconsistent introduction of solid foods in some studies. Studies that compared outcomes in children randomized to soy or cow-milk formula were considered the most reliable, particularly when parents and outcome assessors were masked to formula assignment.

3.3.2 Experimental animal data-Experimental animal studies on developmental effects of soy formula or other soy foods have been carried out in rats, mice, marmosets, monkeys, and pigs. Ten experimental animal studies were found to have utility for the evaluation process. Of these 10 studies, one small study in rats (Hughes et al., 2004) and one study in marmosets (Sharpe et al., 2002; Tan et al., 2006) involved administration of soy milk or infant formula, and only the marmoset study involved direct administration of soy formula to infant animals. A pig study (Li et al., 1990) used a 48\% soybean meal-based liquid diet for neonatal animals. This soybean-meal content is higher than the 14.6-16\% content of soy-protein isolate in marketed formulas (see Section 1.1). Most of the developmental studies carried out in rodents examined the effects of dietary soy products or of soy-isoflavone preparations added to soyfree diets, and it is not clear to what extent the effects of soybean meal are similar to those of commercial isolates. The dietary interventions used in the experimental animal studies differ from one another and it is not clear which, if any, are appropriate models for soy infant formula. Dose-response information is available only from the study of McVey et al. (2004a,b), in which an isoflavone mixture was added to a soy-free feed giving rise to five different isoflavoneintake levels. The use of this study in the assessment of possible toxicity of soy formula assumes that the isoflavone content of soy formula is the toxicologically important component. 


\subsection{Summary of Developmental Toxicity Data}

3.4.1 Human studies-Studies on outcomes after feeding soy formula in infancy are summarized in Table 41. Most of the studies on growth have not shown differences between soy and cow-milk formula in term infants, although most of the studies had small sample sizes (Kay et al., 1960;Dean, 1973;Jung and Carr, 1977;Zoppi et al., 1982;Steichen and Tsang, 1987;Venkataraman et al., 1992;Mimouni et al., 1993;Lasekan et al., 1999). Some studies reported that infants given soy formula have transiently lower bone-mineral content than infants fed cow-milk formula (Köhler et al., 1984) or human milk (Chan et al., 1987); however, other studies reported that serum concentrations of bone accretionrelated hormones and minerals in soy formula-fed infants showed the appropriate compensatory response to the vitamin and mineral content of the food source (Hillman, 1988;Hillman et al., 1988;Mimouni et al., 1993). Reports in very low birth-weight and preterm babies suggest that soy formula may not support adequate growth and bone development in these populations (Naude et al., 1979; Kulkarni et al., 1984). Soy infant formula is not marketed for use in preterm infants.

The relationship between exposure to soy formula and hypersensitivity or allergic disorders has been investigated. Intestinal changes similar to those of gluten enteropathy have been described in infants with soy-sensitivity (Poley and Klein, 1983); however, infants with intestinal hypersensitivity do not seem to react to soy formula more often than they react to cow-milk formula (Burks et al., 1994). Studies comparing soy- and cow milk-formula feeding in infants with familial predispositions to allergic disease have not shown an advantage of one formula over another (Gruskay, 1982; Hillman et al., 1988; Miskelly et al., 1988; Chandra et al., 1989a,b; Chandra and Hamed, 1991; Chandra, 1997, 1998; Klemola et al., 2002), but most of these studies have shown that breast feeding was superior to the use of either formula. The Expert Panel is aware that the validity of the articles by Chandra has been challenged.

Case reports suggest that hypothyroid infants on oral thyroxine therapy fed soy formula may not absorb the thyroxine from the intestine. This effect has been attributed to fecal wastage with decreased enterohepatic circulation (Shepard, 1960; Chorazy et al., 1995; Jabbar et al., 1997). Although the addition of iodine and reduction in fiber content of soy formulas is a partial solution, one study noted that the problem may occur if formula intolerance increases stool frequency (Chorazy et al., 1995).

A case-control study of premature thelarche used 130 subjects from 552 potentially eligible girls (Freni-Titulaer et al., 1986). Age-matched controls were recruited, consisting in some but not all instances of friends of the cases. Parents were interviewed with regard to family history and possible exposures, including the use of soy formula. Multivariate analysis did not show a significant relationship between premature thelarche and soy-formula feeding. When the analysis was restricted to girls with onset of premature thelarche before 2 years of age, a significant association with soy-formula feeding was identified $(\mathrm{OR}=2.7,95 \% \mathrm{CI}=1.1-6.8)$. Other significant factors included maternal ovarian cysts and consumption of chicken. Consumption of corn was protective.

Strom et al. (2001) identified 952 adults who had participated as infants in a controlled, nonrandomized formula trial. There were 248 adults who had been given soy formula and 563 who had been given cow-milk formula. The subjects were asked about height, weight, sexual maturation, education level, and reproductive outcomes. No formula-related differences in height, weight, body-mass index, or sexual maturation were identified. Women who had been given soy formula had longer menstrual periods (mean $=0.37$ days, $95 \% \mathrm{CI}=0.06-0.68$ days) and there was a soy formula-associated increase in the risk of experiencing severe menstrual discomfort ( $\mathrm{RR}=1.7,95 \% \mathrm{CI}=1.04-3.00$ compared to no or mild discomfort, unadjusted). With adjustment for multiple comparisons, these findings were no longer statistically significant (>30 different endpoints were evaluated). 
Another study (Giampietro et al., 2004) evaluated 48 children who had been given soy formula and 18 children who had not been given soy formula and found no evidence of precocious puberty or gynecomastia. The sample size was not adequate to detect an increase in premature thelarche of the size suggested by Freni-Titulaer et al. (1986).

There are two case-control studies of women with breast cancer in which a protective effect of adolescent soy foods was suggested (Shu et al., 2001; Wu et al., 2002). Both studies involved Asian women in either Shanghai or Los Angeles county. Neither study evaluated the use of soy infant formula. A mammography study found soy food intake during the first 19 years of life to be associated with the mammographic identification of more dense breast tissue (Maskarinec et al., 2004b). Use of soy infant formula was not separated from use of other soy foods in this report.

3.4.2 Experimental animal studies-Experimental animal studies are summarized in Table 42, and all were found to be of limited utility for the evaluation process. The only study in which exposure to dietary soy was likely limited to dams was that of Masutomi et al. (2004). Sprague-Dawley rat dams were given a soy-based or a soy-free diet until PND 10, before the time when pups will eat maternal feed. Female offspring in the soy-diet group had an increase in body weight on PND 21 and a decrease in weight-adjusted anogenital distance. Male offspring in the soy-diet group had an increase in body weight on PND 2. The study by Hughes et al. (2004) gave soy milk or rice milk instead of water to lactating rats (10-30 mg/ $\mathrm{kg}$ bw/day) and evaluated offspring at weaning. There were only three dams exposed to soy milk and two dams exposed to rice milk. There was an increase in female pup body weight, a decrease in weight-adjusted female anogenital distance, a decrease in uterine progesterone receptor, and a decrease in relative epididymal weight; however, the litter was not used as the experimental unit in the analysis, limiting the utility of the study. Although treatment was given to the dam, the pups would have had access to the soy milk during the latter part of the lactation period. The body weight increases in offspring in the Masutomi et al. and Hughes et al. studies were not confirmed by Lund et al. (2001a) or Atanassova et al. (2000), who reported a decrease in offspring body weight associated with developmental exposures to soy-based diets, albeit at a later age of evaluation.

Several rodent studies compared soy-based and soy-free diets during pregnancy and lactation with pups weaned to their dam's diet (Mäkelä et al., 1995b; Lund et al., 2001a; Odum et al., 2001; McVey et al., 2004a,b). The effects reported in these studies were inconsistent. Odum et al. (2001) reported advancement of pubertal landmarks in males and females, but Lund et al. (2001a) reported delayed vaginal opening. Testis weight was reported to be decreased by dietary soy (Atanassova et al., 2000) and increased by dietary soy (McVey et al., 2004b). These differences may reflect different isoflavone contents of the diets used in different studies or other differences in the composition of the feed.

The study of McVey et al. (2004a,b) permits a dose-response evaluation of dietary isoflavone supplementation and developmental effects. These investigators used a soy-free diet to which was added soy protein and soy isoflavones at five exposure levels (plus the soy-free diet control). The most sensitive endpoint was an increase in testis weight on PND 28 in rats that were exposed during pregnancy, lactation, and weaning to a dietary-isoflavone intake $\geq 2 \mathrm{mg} /$ $\mathrm{kg}$ bw/day. The increase in testis weight did not show a dose-response relationship.

The study of Sharpe et al. (2002), extended by Tan et al. (2006) involved the feeding of marmosets co-twins with either soy infant formula or a cow-milk formula. The authors expected the use of co-twins to permit paired analyses and to decrease variability between animals assigned to different treatments. The study authors reported a decrease in plasma testosterone on PND 35-45 with an increase in testis weight and in numbers of Sertoli and 
Leydig cells at 120-138 weeks of age. The authors expressed concern that because the marmosets were only partially formula-fed, effects in human infants who are exclusively formula-fed may be more severe. Interpretation of this study was limited in part by the lack of information on normal variability in the reported parameters in marmosets and by concern that the marmoset may not be an appropriate model for assessing human risk.

\section{Conclusions of the Expert Panel}

Evidence is insufficient to conclude that soy infant formula would or would not produce developmental toxicity with exposure during pregnancy. There are no data from exposure during pregnancy only in humans or experimental animals that are relevant to this evaluation.

Evidence is insufficient to conclude that soy infant formula would or would not produce developmental toxicity with childhood exposure.

Evidence is insufficient to conclude that soy infant formula would or would not produce developmental toxicity in animals.

Note: The definitions of the term sufficient and the terms assumed relevant, relevant, and not relevant are in the CERHR guidelines at http://cerhr.niehs.nih.gov/news/guidelines.html.

\subsection{REPRODUCTIVE TOXICITY DATA}

\subsection{Human Data}

4.1.1 Female-A number of studies examined the effects of dietary soy intake on hormone levels or menstrual cycles of women. The studies are presented in order of publication.

Cassidy et al. (1994, 1995), in studies funded by NIH and the U.K. Ministry of Agriculture, Fisheries, and Food, studied the hormonal effects of isoflavone intake in premenopausal women. Subjects included 15 healthy, non-vegetarian women aged 20-29 years. The women tracked their menstrual cycles and measured body temperatures to determine if ovulation was occurring for 4 months before the study. The women were then moved into metabolic suites and fed control diets with no soy products for the duration of one menstrual cycle. Beginning on or before the first day of menses and continuing through the next menstrual cycle, the women were fed diets containing isoflavones in various forms. A period of 1-4 months separated the control and soy product-exposed dietary periods. In most cases, diet administration was not randomized. One group of six women received $60 \mathrm{~g} /$ day textured-vegetable protein, containing $45 \mathrm{mg}$ conjugated isoflavones (mean $\pm \mathrm{SD}$ daidzein $25.08 \pm 0.31 \mathrm{mg}$ /day and genistein 19.85 $\pm 0.43 \mathrm{mg}$ /day) in place of beef, chicken, or cod. [Based on the mean body weight of $61.6 \mathrm{~kg}$, intake of conjugated isoflavones was $0.73 \mathrm{mg} / \mathrm{kg}$ bw/day, daidzein was $0.41 \mathrm{mg} / \mathrm{kg} \mathrm{bw/}$ day, and genistein was $0.32 \mathrm{mg} / \mathrm{kg}$ bw/day.] A group of 6 women received miso $50 \mathrm{~g} /$ day, containing $25 \mathrm{mg}$ unconjugated isoflavones $[0.42 \mathrm{mg} / \mathrm{kg}$ bw/day isoflavone based on a mean body weight of $59.0 \mathrm{~kg}$ ] in place of beef, chicken, or cod. Three of the subjects dropped out of the study because they could not tolerate the high salt levels in miso. A group of five subjects received $60 \mathrm{~g}$ Arcon F, an isoflavone-free soy product, administered in biscuits or breads. Diets were randomized in Study 3. One group of six women was given $28 \mathrm{~g}$ textured vegetable protein, with $23 \mathrm{mg}$ isoflavone $[0.40 \mathrm{mg} / \mathrm{kg} \mathrm{bw} / \mathrm{day}$ isoflavone based on mean body weight of $57.0 \mathrm{~kg}$ ] in place of part of their bread. Isoflavone intake levels were based on phytoestrogen intakes in Asian diets, reported at 50-100 mg/day. Blood and 24-hr urine samples were collected every 3 days during each dietary period. Blood was also collected on the day of ovulation, as determined by examining urine with an ovulation-detection kit. Plasma sex hormone-binding globulin, progesterone, 17 $\beta$-estradiol, LH, and FSH levels were measured 
by RIA in the first study and by time-resolved fluorometry in the remaining three studies. Plasma cholesterol levels were measured. Intestinal transit times were determined by measuring radiolabel in feces. Statistical significance of results was analyzed by paired $t$-test. Urinary levels of isoflavones were reported for the earlier study (Cassidy et al., 1994) and those results are discussed in Section 2.1.1.3.

Subjects maintained constant body weights during the study. The only dietary-related effect on mean intestinal transit time was an increase during the follicular phase in subjects fed miso. The majority of menstrual and hormonal effects were observed in the $45 \mathrm{mg} /$ day conjugatedisoflavone group, summarized in Table 43. Length of the follicular phase was increased in subjects fed $45 \mathrm{mg} /$ day conjugated isoflavones, mid-cycle levels of LH and FSH were reduced, and $17 \beta$-estradiol levels during the follicular phase were increased. However, as noted in the earlier study (Cassidy et al., 1994), the reductions in the LH and FSH surge may have been due to sampling time, because no special attempt was made to determine the exact day of ovulation, and peak plasma and urinary concentrations may have been off by 1 day. Time to peak progesterone and peak progesterone concentrations were significantly reduced in the miso group, but the study authors concluded that the group size $(n=3)$ was too small to draw conclusions. In the earlier study (Cassidy et al., 1994), overall menstrual cycle length was increased by 3 and 5 days in two subjects whose urinary excretion of equol was high (see Section 2.5 on potentially susceptible subpopulations). No other effects were observed on menstrual cycles or plasma hormone levels. Cholesterol levels were reduced with intake of 45 but not $23 \mathrm{mg} /$ day conjugated isoflavones. An increase in cholesterol levels occurred in the subjects fed isoflavone-free soy.

Strengths/Weaknesses: The use of a controlled environment for the diet intervention is a strength of this study, as is the use of subjects as their own controls. The age range of the women was narrow, and the same batches of food and soy protein were used throughout the study. The use of three different sources of soy is also a strength. The hormone measures were

appropriately selected, and there were good internal quality controls for the assays. Adequate blood, urine, and fecal samples were collected. The paired statistical methods were appropriate, but it is not clear if the hormone data were normally distributed and, if not, if the appropriate adjustments were made. Good data on menstrual history were not collected, and it was not clear that potential confounders such as ethnic origin, body-mass index, alcohol, and smoking were considered in the hormone evaluations. Past oral contraceptive use was not considered. The small sample size and high drop-out rate on the miso diet are weaknesses and are likely to have limited the ability to control for potential confounders. The lack of randomization of diet order is a problem, and data were not presented in a manner permitting an evaluation of possible effects of diet order. This study at best evaluates acute effects for one cycle, and no information is available on whether these effects would have been sustained.

Utility/Adequacy for CERHR Evaluation Process: This study is useful, with the limitations noted.

Lu et al. (1996), supported by the Public Health Service, evaluated the effect of soy milk supplementation on menstrual cycle hormones in 6 healthy non-vegetarian women with regular menstrual cycles. The subjects were admitted to a hospital clinical research center for 33 days, during which time they were served a hospital diet with three $12 \mathrm{oz}$ portions of soy milk (one portion with each meal). The soy milk was assayed periodically for isoflavone content by GC and contained a mean $\pm \mathrm{SD}$ of $33.49 \pm 14.48 \mathrm{mg} / 12 \mathrm{oz}$ daidzin/daidzein and $38.39 \pm 14.62 \mathrm{mg} /$ $12 \mathrm{oz}$ genistin/genistein. [The aglycones were not reported separately but were estimated as $15-19 \%$ of the total isoflavone content.] Blood was sampled 1 day before and 1 day after the initiation of soy milk, and weekly thereafter for 1 month. Three subjects had blood sampled during the month before initiation of soy milk, and all subjects had blood samples taken during 
at least three of six cycles that followed the cessation of soy-milk supplementation. Four subjects were followed up to 10 months. [Time points reported are baseline, during soymilk ingestion, 1 month after soy-milk ingestion, and 2-3 months after soy-milk ingestion. Hormone levels from the 1 month before soy milk and for the 5-10 months after ingestion were not different and were averaged to produce the "baseline" values.] Serum assays were carried out by RIA for $17 \beta$-estradiol, progesterone, and dehydroepiandrosterone sulfate. Menstrual cycle length was recorded, and serum levels of $17 \beta$-estradiol and progesterone were reported by cycle phase (day 5-7, day 12-14, day 20-22). Statistical analysis was carried out by Friedman 2-way ANOVA. If duration of treatment affected hormone levels, multiple comparisons using paired t-tests were carried out on unranked data. [Based on the way the results were presented, it seems that "duration of treatment" refers to the time block with respect to soy ingestion. The paired $t$-tests seem to have been carried out using the baseline hormone concentration as the control in each pair. There was no control for possible effects of hospitalization on hormone concentrations.] Results are summarized in Table 44. The authors concluded that 1 month of soy supplementation increased menstrual cycle length and decreased $17 \beta$-estradiol serum levels. They postulated that the decrease in dehydroepiandrosterone sulfate may reflect a decrease in $17 \beta$-estradiol synthesis because dehydroepiandrosterone sulfate is a precursor in 17ß-estradiol synthesis. [The Expert Panel notes that the sulfate is considered to be an adrenal product rather than a reflection of ovarian steroid synthesis.] The authors contrasted their results with those of Cassidy et al. (1994), noting that women in the Cassidy et al. study had an increase in follicular-phase levels of $17 \beta$-estradiol and no change in this hormone at mid-cycle or in the luteal phase. They speculated that the larger amount of isoflavone supplementation in this study compared to that of Cassidy et al. may have been responsible for the difference in results.

Strengths/Weaknesses: This study included good information about the components of the soy supplementation and good methods of hormone measurement. The controlled environment for the diet intervention, the use of subjects as their own controls for a portion of the analysis, and the use of at least two cycles of follow-up after the intervention are additional strengths. Weaknesses include the small sample size, varying lengths of soy ingestion, varying numbers of cycles for follow-up, missing values at some time points, and lack of control for potential confounders. Women on oral contraceptives should have been excluded or should have had a complete washout period.

Utility (Adequacy) for CERHR Evaluation Process: This study is useful for the evaluation process.

Nagata et al. (1997) examined the relationship between soy intake and blood levels of $17 \beta$ estradiol and sex hormone-binding globulin. Fifty healthy women (21-42 years old) were questioned about menstrual and reproductive history and dietary intake of 169 foods, including nine soy foods. A blood sample was taken on Days 11 and 22 of the menstrual cycle (first day of menstrual bleeding = Day 1) for measurement of serum $17 \beta$-estradiol and sex hormonebinding globulin. Data were logarithmically transformed and adjusted using the Willett method, and Spearman rank correlation coefficients were calculated. After adjusting for age, body mass index, cycle length, and energy intake, a significant negative association was found between soy intake and 17 $\beta$-estradiol levels on Day $11(r=-0.33)$ and Day $22(r=-0.30)$ of the cycle; the Day-22 association remained significant after additional adjustments for fat and crude-fiber intake. No association was detected between total soy intake and sex hormonebinding globulin. However, a significant negative association $(r=-0.36)$ was noted between miso intake and sex hormone-binding globulin on Day 22, after adjustment for age, body-mass index, cycle length, and energy, fat, and fiber intake. 
Strengths/Weaknesses: This study involved healthy subjects with no oral contraceptive or other hormone use. The broad assessment of dietary intake under natural conditions and adjustment for some potential confounders are strengths of the study. The use of detailed questionnaire information and appropriate transformation of hormone data are additional strengths. The sample size was, however, relatively small with a wide range of ages. The food frequency questionnaire covering previous-year average intake may not have accurately represented intake. Isoflavone levels were not measured, only estimated from reported food intake. The use of an exclusively Japanese sample may limit generalizability.

Utility (Adequacy) for CERHR Evaluation Process: This study is useful for the evaluation process.

Nagata et al. (1998) examined the effects of soy intake on menstrual cycle length and hormone levels in a study conducted at a Japanese university. Study subjects consisted of healthy premenopausal women who were either students or teachers at a nursing school. Subjects were randomly assigned to a soy-milk group $(n=31)$ or control group $(n=29)$. There were no significant differences in age, weight, height, body-mass index, age at menarche, parity, or smoking status between groups. Subjects in the soy-milk group were instructed to drink 400 $\mathrm{mL} /$ day of soy milk that was supplied to them, and only that soy milk. The soy milk contained $109 \mathrm{mg}$ isoflavones. [Based on actual mean weights of subjects, $\sim \mathbf{5 0} \mathbf{~} \mathbf{~ g}$, total isoflavone intake is estimated at $2.2 \mathrm{mg} / \mathbf{k g ~ b w} / \mathbf{d a y}$.] The study was conducted over three menstrual cycles with soy-milk supplementation beginning on Day 11 (first day of menstrual bleeding = Day 1) of the first cycle and ending on Day 11 of the third cycle. Women in the soy-milk and control groups were instructed to continue eating their regular diets. Women were asked to keep dietary records, which were used to estimate intake of soy products, macronutrients, micronutrients, and isoflavones. Blood samples were collected as close as possible to Day 11 (actual collection occurred on Days 9-12) of Cycles 1 and 3 for analysis of estrone, 17 $\beta$ estradiol, and sex hormone-binding globulin. Baseline and final values of all parameters were compared in the soy-milk and control groups. Statistical analyses included ANOVA to assess cycle-length changes and Mann-Whitney and Wilcoxon matched pairs signed rank tests to analyze hormone effects.

Diets in the soy-milk and control groups were stated to be almost identical in terms of macronutrient, micronutrient, soy, and isoflavone intakes. [There was no statistical evaluation of dietary differences between treatment groups.] Significant dietary changes occurring in both groups over the course of the study included decreased intake of calories, cholesterol, and carbohydrate. Intake of fat and protein was also reduced significantly in the control group. Variable changes in micronutrient intake were noted in both groups. Mean soymilk intake in the group given soy milk was estimated at 354.8-365 mL. In the soy-milk group, total isoflavone intake [assumed to be daily intake] from all dietary soy sources was about 4.5 times higher at the end (mean $=116.4 \mathrm{mg}$ ) compared to the beginning of the study (mean $=25.4 \mathrm{mg}$ ). [The final value of total isoflavone intake was made up of isoflavone intake from soy milk: mean $\pm \mathrm{SD}=\mathbf{9 7 . 0} \pm \mathbf{1 9 . 2}$ and other soy products: mean $\pm \mathrm{SD}=19.4 \pm 15.0$.] The authors mentioned that isoflavone intake divided by energy intake was not significantly affected in the control group. [However, statistical significance was obtained for a slight reduction in total isoflavone intake from other soy products at the end (mean $\pm \mathrm{SD}=18.4$ $\pm 13.4 \mathrm{mg}$ ) compared to the beginning (mean $\pm \mathrm{SD}=20.7 \pm 12.9 \mathrm{mg}$ ) of the study in the control group.] The third and fourth menstrual cycles were about 2 days longer in the soymilk group but 1-2 days shorter in the control group compared to the first menstrual cycle; statistical significance was not attained in either group for comparisons of within-group cycle lengths. Differences between groups were also stated to be non-significant. [It is not clear how this determination was made.] The only significant effect on hormone level was a $23 \%$ reduction in final compared to initial serum estrone level in the soy-milk group. 
Statistical analyses were repeated after restrictions to include only data from women providing blood samples no more than 1 day apart in Cycles 1 and 3 (e.g., Day 11 in Cycle 1 and Day 10 in Cycle 3). The restricted dataset was comprised of data from 21 women in the soy-milk group and 23 women in the control group. Isoflavone intakes for the restricted datasets were similar to intakes obtained for the larger dataset. Compared to the first menstrual cycle, the fourth menstrual cycle was about 3.4 days longer in the restricted soy-milk group and 2.6 days shorter in the restricted-control group. The only significant effect on hormone level in the restricted groups was a $\sim 30 \%$ reduction in final compared to initial serum estrone level in the soy-milk group. The study authors concluded that larger studies are needed to confirm the findings of this study.

Strengths/Weaknesses: The random assignment to intervention, the use of subjects as their own controls, and the use of three menstrual cycles are strengths of this study. The control and treatment groups were similar at baseline, and the statistical analyses were appropriate. The sample size was small, although the sample size calculation carried out by the authors suggested adequate power for the study. Compliance was not assessed objectively, for example, with urine measurements. There were substantial changes in reported dietary composition over the course of the study, and the changes differed in the intervention compared to the control group. These changes were not accounted for in the paired analyses or group analyses. The study would have been strengthened with the use of multiple samples for hormone measurements throughout the cycle.

Utility (Adequacy) for CERHR Evaluation Process: This study is useful for the evaluation process.

Xu et al. (1998), supported by NIH, evaluated the effects of dietary soy on estrogen metabolism in 12 regularly cycling women aged $26.0 \pm 4.9$ (SD) years. The subjects were studied on each of three different commercial soy-powder supplements in a randomized cross-over design. The three supplements were designed to give a mean daily total isoflavone intake of $0.16,1.01$, or $2.01 \mathrm{mg} / \mathrm{kg}$ bw. Each supplement period consisted of three menstrual cycles plus 9 days of a fourth menstrual cycle, with about a 3-week unsupplemented washout period between treatment periods. The isoflavone content of each supplement consisted of about 55\% genistein, $37 \%$ daidzein, and $8 \%$ glycitein, mostly as their glycoside conjugates. During Days 7-9 of the fourth menstrual cycle in each treatment period, three consecutive 24-hr urine samples were collected and analyzed for phytoestrogens and for endogenous estrogens and their metabolites. Data were analyzed using ANOVA [post-hoc test not specified] to compare urinary values between treatments, taking the $0.16 \mathrm{mg} / \mathrm{kg}$ bw/day isoflavone treatment as the control. Urinary excretion of total genistein increased from a mean of $997 \mathrm{nmol} / 24 \mathrm{hr}$ in women on the control treatment to 6529 and $14,200 \mathrm{nmol} / 24 \mathrm{hr}$ in women on the higher-isoflavone supplements. Total daidzein excretion increased from a mean of $995 \mathrm{nmol} / 24 \mathrm{hr}$ in women on the control supplement to 4964 and $9528 \mathrm{nmol} / 24 \mathrm{hr}$ in women on the higher-dose supplements. Other isoflavones and lignans also increased in urine with increasing isoflavone content of the supplement. Urinary estrone and $17 \beta$-estradiol and their metabolites decreased with increasing isoflavone supplementation; 17ß-estradiol excretion was reduced 15-23\% and estrone excretion was reduced $8-15 \%$ by the 2 higher-isoflavone soy supplements compared to the control supplement. Metabolites of these estrogens were also reduced by isoflavone supplementation. [The reduction of some of the hydroxylated estradiol and estrone metabolites was the focus of this report, which dealt with possible mechanisms of breast cancer risk reduction.] The authors noted that the main study, of which this substudy was a part, did not show alterations in plasma estrogens or in the menstrual cycle (Duncan et al., 1999a). They proposed that urinary excretion of endogenous estrogens and their metabolites may be a more sensitive indicator of dietary effects on reproductive hormones than are plasma estrogens. 
Strengths/Weaknesses: The randomized cross-over design, clear inclusion and exclusion criteria, range of dose levels, treatment over three menstrual cycles, appropriate statistical analyses, and evaluation of other sources of dietary isoflavones were strengths of this study. The control and treatment groups were similar at baseline, and the controls had minimal exposure to soy products. Phytoestrogens were measured in urine for an objective evaluation of soy ingestion. The small sample size, however, made it impossible to adjust for dietary and other differences between groups, and the study may not have been adequately powered to detect important differences between groups. It is not clear if the randomized block design provided random ordering of treatment groups. There was a lot of variability in the urinary levels of phytoestrogens.

Utility (Adequacy) for CERHR Evaluation Process: This study is useful for the evaluation process.

Martini et al. (1999), supported by the Minnesota Soybean and Research Council and the Minnesota Agricultural Experimental Station, studied menstrual cycle length and serum hormone measurements in 16 regularly cycling women who were not on oral contraceptives and 20 oral contraceptive users. Women were randomized to receive daily treatments consisting of 10 ounces of skim milk with or without a commercial soy supplement for two consecutive menstrual cycles. After the 2-month treatment, subjects were crossed over to the other treatment. The $34 \mathrm{~g}$ daily serving of the soy supplement contained $38 \mathrm{mg}$ total isoflavones, including $23 \mathrm{mg}$ genistein and $13 \mathrm{mg}$ daidzein. Women who were not on oral contraceptives collected 24-hr urine samples and had fasting blood drawn on two consecutive days in the midfollicular phase and the mid-luteal phase (timed using a urinary LH surge-detection kit). Oral contraceptive users had the same evaluations during two consecutive days between cycle Days 7 and 9 and cycle Days 21 and 23. Serum assays included commercial RIA for 17 $\beta$-estradiol, estrone, dehydroepiandrosterone sulfate, and sex hormone-binding globulin, and commercial chemiluminescence immunoassays for progesterone and prolactin. Urine enzyme

immunoassays were carried out for 2- and 16 $\alpha$-hydroxyestrone, estrogen metabolites that have been associated with breast cancer risk modification. Statistical analysis was carried out using paired $t$-tests, with each woman serving as her own control. No effects of soy supplementation were detected on serum or urine measurements or on lengths of the menstrual cycle or its component phases. The authors called attention to their results differing from those of previous investigators (Cassidy et al., 1994, 1995; Lu et al., 1996), and suggested that hormone measurements may not be very precise unless stable isotope-dilution MS is used. The authors concluded that their results suggested a minimal influence of dietary soy on fertility and no interaction with oral contraceptives.

Strengths/Weaknesses: The randomized cross-over design with subjects serving as their own controls is a strength. The statistical analyses were appropriate; however, an intention-to-treat analysis should also have been carried out. The inclusion of an oral contraceptive-using group and the treatment over two menstrual cycles are additional strengths. Weaknesses include the small sample size, broad age range, and lack of control for potential confounders. The wide range of ages may be particularly problematic because cycle characteristics in 40-year-old women may be different from those in 18-year-old women. There was large variability in the oral-contraceptive formulations, which may have influenced the results. There does not seem to have been a washout period between treatments. The authors dropped two women from the analysis without solid reasons.

Utility (Adequacy) for CERHR Evaluation Process: This study is useful for the evaluation process. 
Duncan et al. (1999a), in a study funded by NIH and the National Center for Research Resources, studied the effects of isoflavone intake on hormone action in 14 healthy, nonvegetarian premenopausal women (mean age $\pm \mathrm{SD}=26.5 \pm 4.7$ years). Using a randomized crossover design, subjects received one of three soy-protein powders in addition to consuming their regular diets. They were instructed to avoid foods high in phytoestrogens, alcohol intake, and vitamin and mineral supplementation. Each of the diets provided for total isoflavone intake of $0.15 \pm 0.01$ (control), $1.01 \pm 0.04$ (low), or $2.01 \pm 0.03$ (high) $\mathrm{mg} / \mathrm{kg}$ bw/day. The dietary isoflavones (expressed as the aglycones) included 55\% genistein, 37\% daidzein, and $8 \%$ glycitein. Ninety-seven percent of genistein and daidzein and $91 \%$ of glycitein were present

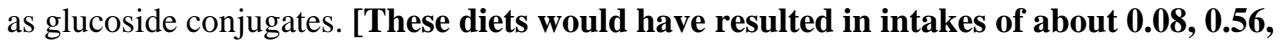
and $1.1 \mathrm{mg} / \mathrm{kg}$ bw/day genistin + genistein; $0.06,0.37$, and $0.74 \mathrm{mg} / \mathrm{kg}$ bw/day daidzin + daidzein, and $0.012,0.08$, and $0.16 \mathrm{mg} / \mathrm{kg}$ bw/day glycitin + glycitein in the control through high dose groups, respectively.] Doses were selected to represent ranges of isoflavone intakes from typical Asian diets. Beginning on Day 2 of the menstrual cycle, subjects consumed each diet through three menstrual cycles and an additional 9 days. A washout period of 3 weeks separated each diet. Subjects determined the occurrence of ovulation using home kits. Blood samples were collected during Cycles 2 and 3 of each dietary period to determine levels of estrogens, androgens, progesterone, cortisol, thyroid hormones, insulin, prolactin, sex hormone-binding globulin, LH, and FSH. Hormones were measured by RIA, with the exception of LH and FSH, which were measured by immunoradiometric assay. Data were analyzed by ANOVA.

A review of dietary records showed that soy-protein supplementation significantly increased calorie and protein intake and reduced fiber intake compared to pre-study levels. There were no detected changes in body weight, body-mass index, or percent body fat. Cycle lengths were recorded for the second and third cycle of each dietary period to allow subjects to adapt to each diet. No significant effects of isoflavone intake were detected on total menstrual cycle length, follicular phase length, or luteal phase length. There was a significant reduction in estrone [by 13\%] for the high- compared to the low-isoflavone diet during the mid-follicular stage.

Significant reductions in LH [by 28\%] and FSH [by 15\%] for the low-isoflavone compared to control diet occurred during the peri-ovulatory period. Consistent with the plasma data for $\mathrm{LH}$, there was a "nonsignificant trend toward decreased excretion with increased isoflavone consumption." ["Nonsignificant trend" was not defined, and data were not shown.] Also observed with intake of high-isoflavone diet were significant reductions in plasma free triiodothyronine [by 5.5\%] compared to the control group and dehydroepiandrosterone sulfate [by 6.7\%] compared to the low-isoflavone group. Due to a lack of effect on thyroxine and thyroid-stimulating hormone, the authors did not expect the change in triiodothyronine levels to be physiologically important. No effects were noted for the other hormones analyzed, including $17 \beta$-estradiol and progesterone. Endometrial biopsies were conducted during the third menstrual cycle of each dietary-exposure period, and a comparison between expected and actual histologic results identified no dietary effects [data were not shown]. According to study authors, the results of this study suggested weak hormonal effects in the absence of doseresponse relationships.

Strengths/Weaknesses: The randomized cross-over design and clear exclusion criteria are strengths. There was a range of dose levels, permitting the evaluation of dose-response relationships, and treatment over three menstrual cycles was preceded by a washout period. The use of free-living women may improve generalizability. The repeated-measures analysis is an additional strength. Weaknesses include the small sample size, potential selection/ recruitment bias, and the discarding of Cycle 1 data without a solid rationale. It is not clear that a 3-week washout period between interventions was adequate. The authors did not indicate how many women were excluded based on not having adequate ovulation data. There was also no mention of the normality of the hormone data and whether transformations were used. 
Utility (Adequacy) for CERHR Evaluation Process: This study is useful for the evaluation process.

Watanabe et al. (2000), supported by the Japanese Ministry of Health and Welfare and the Science and Technology Agency, evaluated effects of soy supplementation on Japanese women. [Ages not given, except that subjects were young premenopausal women and that they were students in a nutrition course.] The subjects were instructed to consume isoflavones $20 \mathrm{mg} / \mathrm{day}$ from food and were observed for at least two menstrual cycles [one cycle according to the Results section] before assignment to an isoflavone supplement for one menstrual cycle. Diet records showed that actual dietary total isoflavone intake averaged $10 \mathrm{mg} /$ day (range $=4.1-28.9 \mathrm{mg} /$ day). The isoflavone supplement was given in tablet form with meals. Dose groups included a control group $(n=20)$, isoflavones $20 \mathrm{mg} / \mathrm{day}$, and isoflavones $40 \mathrm{mg} /$ day ( $\mathrm{n}=19$ or 20 for the supplement groups combined). [It is not specified whether the control group took a placebo tablet. The isoflavone tablets were described as containing $43.5 \mathrm{mg}$ daidzein, $6.0 \mathrm{mg}$ genistein, and $24.0 \mathrm{mg}$ glycitein, making it difficult to understand how the subjects took daily isoflavone doses of 20 and $40 \mathrm{mg}$. The authors described the calorie content of the tablets as "per gram tablet." If they meant also to describe the isoflavone content as per gram tablet, each $200 \mathrm{mg}$ tablet would have contained $8.7 \mathrm{mg}$ daidzein, $1.2 \mathrm{mg}$ genistein, and $4.8 \mathrm{mg}$ glycitein, or $15.7 \mathrm{mg}$ total named isoflavones.] Subjects were observed for an additional one or two menstrual cycles after the supplementation period.

Urinary excretion of total daidzein, genistein, and glycitein was measured before the supplement period and on Day 30 of the supplement period. Blood samples were collected "on Day 1 of the study and on 11th and 22nd day after the menstruation, and at the end of the experimental period." [It cannot be determined if blood samples were collected during each menstrual cycle, which would be a total of two before the supplemented cycle, one during the supplementation period, and one or two after the supplementation period, or only during the supplementation period. The authors indicated that the Day 11 and Day 22 samples did not correlate well with follicular and luteal phases because "half the students had had rather irregular menstruation cycles."] Plasma 17 $\beta$-estradiol and progesterone were measured by RIA or by fluorescence immunoassay. An additional three students with regular menstrual cycles were evaluated for one baseline menstrual cycle and one cycle during which they took supplemental isoflavones $50 \mathrm{mg} /$ day. Blood samples were collected every day during the ovulatory period [not otherwise specified] and 3-5 times each during the follicular and luteal phases, which were identified using basal body-temperature monitoring. Plasma was used for measurement of estrone, 17 $\beta$-estradiol, estriol, sex hormone-binding globulin, LH, FSH, androstenedione, testosterone, triiodothyronine, and thyroxine. [An additional hormone, identified as "epiandeostendione," was measured. The authors may have meant dehydroepiandrosterone.] FSH and LH were determined using time-resolved immuno-fluorometric assays, and the other hormones were determined using RIA. [Statistical methods were not explained except for use of the Pearson correlation to evaluate relationships between isoflavone excretion and plasma hormone levels. It could not be determined whether supplemented cycles were compared to baseline cycles within subjects or supplemented subjects were compared to control subjects.]

Urinary daidzein was reported to have increased with both supplement levels, and urinary genistein was reported to show a "slight increase" in the group given $40 \mathrm{mg} /$ day supplemental isoflavones. [Statistical testing was not mentioned.] Plasma 17 $\beta$-estradiol was reported to have increased by $66 \mathrm{pg} / \mathrm{mL}$ in the luteal phase and $20 \mathrm{pg} / \mathrm{mL}$ in the follicular phase in women given isoflavone supplementation $20 \mathrm{mg} / \mathrm{day}$, and by $97 \mathrm{pg} / \mathrm{mL}$ in the luteal phase and $25 \mathrm{pg} /$ $\mathrm{mL}$ in the follicular phase in women given isoflavone supplementation $40 \mathrm{mg} / \mathrm{day}$. Plasma progesterone results and comparisons were not given, although the authors wrote, "almost half 
the subjects showed a low response to progesterone after ovulation." There was no correlation between isoflavone excretion and follicular or luteal plasma hormone concentrations except for a positive correlation between daidzein excretion and luteal-phase plasma $17 \beta$-estradiol. Among isoflavone-supplemented women, $60 \%$ had menstrual cycle lengthening, and $20 \%$ had menstrual cycle shortening [presumably compared to baseline cycles]. Among the three students studied in detail before and during the use of 50-mg isoflavone supplements, the cycle was 7 days shorter on supplements in one subject and 4 days longer in the other two subjects. In the woman with the shorter cycle, plasma $17 \beta$-estradiol, LH, and FSH were decreased. Sex hormone-binding globulin was lower in all three women during the supplement period compared to before the supplement period.

The authors concluded that the "observed decreases in ovarian steroid levels" agreed with epidemiology studies suggesting that Asian populations with high-soy intake have lower levels of ovarian steroids and lower incidences of breast cancer than populations with low-soy intake.

Strengths/Weaknesses: The use of isoflavone tablets to control exposures, the use of two dose levels, and the use of urinary measurements to estimate exposures are strengths. Some information was provided on genistein half-life. Weaknesses include the small sample size, the lack of age information for subjects, and uncertainty about whether control subjects took a placebo. The method of assignment to groups is unclear but does not seem to have been randomized. There was inadequate attention to other dietary sources of isoflavones. The only measure of ovulation was body temperature, which is not adequate. No information was provided on assay variability. Statistical analysis was poor, and there was no mention of controlling for potential confounders. Cycle data were poor, and hormone values were associated only crudely with a particular phase of the cycle. The use of subjects with irregular cycles makes interpretation difficult. In general, the presentation of the methods and results was confusing.

Utility (Adequacy) for CERHR Evaluation Process: This study is of some utility in the evaluation process.

Lu et al. (2000a), supported by NIH and the Public Health Service, examined the effects of soy consumption on menstrual cycle lengths and hormone levels. Ten healthy women (ages 23-42 years) with regular menstrual cycles completed the study. Seven of the subjects were Caucasian and three were African-American. Nine of the subjects were nulliparous. During a 3-month baseline period, the women consumed their regular diets. Soy was not a regular part of the usual diets, and the women were instructed to avoid soy intake during the baseline period. Menstrual cycle lengths were monitored throughout the study, and the first day of menstrual bleeding was considered Day 1 of the cycle. Subjects were admitted to a metabolic unit for a soy diet. They consumed the soy diet during one menstrual cycle, starting on Day 2 and continuing through Day 2 of the next cycle. Diets consisted of soy and non-soy foods and were designed to maintain body weight. For dinner each evening, the subjects ingested 36 ounces of soy milk under direct supervision. A GC method was used to measure isoflavone levels in soy milk, and it was determined that the soy milk provided daily doses of $68.8 \pm 3.7$ (SEM) $\mathrm{mg}$ daidzein and $85.2 \pm 5.4 \mathrm{mg}$ genistein. During the first month of the baseline period, blood was drawn on cycle Days 5 (follicular phase), 12 (mid-cycle), and 22 (luteal phase). In the second month of the baseline period and during the soy-diet period, blood was collected on cycle Days 5 and 7 , and then daily from Day 9 of the cycle to Day 2 of the next cycle. Serum [also described as plasma later in study] was analyzed for ovarian hormones by RIA and for gonadotropins using an immunoradiometric method. Serum isoflavone levels were measured by ELISA. Day of ovulation was determined by body temperature. Two 12-hr urine samples were collected daily from cycle Day 2 of the soy cycle to Day 2 of the next cycle. Urine was analyzed for daidzein and genistein by GC. Hormone levels were expressed as AUC, mean daily level, peak 
level, and rates. Within-subject changes were analyzed by paired $t$-tests and Wilcoxon ranked sign tests. Secondary multiple regression analyses were conducted to determine associations between variables.

Compared to regular diets, the soy diets provided significantly more carbohydrate but less protein and fiber. No significant changes in body weight or bodymass index were detected. Urinary excretion of isoflavones is summarized in Section 2.1.1.3. Fifteen hours after soy-milk ingestion, the mean serum level of genistein was reported at $0.85 \mu \mathrm{g} / \mathrm{mL}[3.1 \mu \mathrm{M}]$ and daidzein at $2.95 \mu \mathrm{g} / \mathrm{mL}$ [11.6 $\mu \mathrm{M}]$. The soy diet had no significant effect on menstrual cycle length, but there was a $6 \%$ decrease in length of mean luteal-phase length $(P=0.07)$. Decreased lutealphase length during the soy-diet period was positively and significantly associated with intake and excretion of genistein and daidzein and with decreased protein intake. During the soy-diet period, $17 \beta$-estradiol serum AUC was significantly reduced (24\%). Reductions in 17ß-estradiol levels were positively associated with plasma and urinary levels of daidzein and genistein, an association influenced by age. Reductions in $17 \beta$-estradiol levels were inversely related to protein intake. Serum progesterone levels were significantly reduced by about $45 \%$ during the soy-diet period. The reduction in serum progesterone was inversely associated with isoflavone intake and positively associated with energy and fiber intake. [Results of regression analyses were not clearly reported.] No significant effects of the soy diet on serum LH or FSH were detected. The study authors concluded that soy-induced effects on ovarian hormones were not mediated by gonadotropins; decreases in ovarian hormones may have been related to isoflavones and to intake of energy, protein, and fiber.

Strengths/Weaknesses: The longitudinal study design with clear inclusion and exclusion criteria, use of subjects as their own controls, collection of baseline dietary data, and the control for daily energy and nutritional intakes are strengths. There was, however, no mention of a washout period before study entry. Ovulation was monitored using basal body temperature, which is not adequate. There was a high drop-out rate (10 of 26), and different subjects ingested different soy preparations, which could have caused variability. Confounders such as bodymass index, ethnic origin, smoking, and alcohol, which may have affected hormone levels, were not considered, and given the small sample, a multivariate analysis would not have been possible.

Utility (Adequacy) for CERHR Evaluation Process: This study is useful for the evaluation process.

Lu et al. (2001), supported by NIH, evaluated the effects of a reduced-isoflavone soy preparation on menstrual cycle hormones in nine healthy women. Subjects were regularly cycling omnivorous women between the ages of 26 and 42 years with body-mass indices of $25-33$. Women were evaluated on their usual diets, with instructions not to consume soy foods, after which they were given a diet containing a 36-ounce serving of a beverage prepared with soy powder from which $>99 \%$ of isoflavones had been removed by alcohol extraction. The authors indicated that the $37.9 \mathrm{~g}$ soy-protein content of this beverage was at the upper end of the dietary soy-protein content of Asian diets. The soy-containing diet was designed to maintain body weight and had the same energy content as the usual diet. The cycle on the usual diet and the cycle on the soy diet were monitored using blood sampling on cycle Days 5 and 7 and then daily from cycle Day 9 through Day 2 of the next menstrual cycle. Serum was used for measurement of $17 \beta$-estradiol and progesterone by RIA and FSH and LH by a commercial immunoradiometric assay. Sex hormone-binding globulin was evaluated using a commercial time-resolved fluoroimmunoassay. Hormones were evaluated using AUC, mean concentration, and peak concentration. Cycle lengths were also compared between the 2 diet conditions. Analyses were carried out by menstrual cycle phase (follicular and luteal, identified by measurement of the LH surge) and over the whole cycle. Comparisons were made using 
ANCOVA with a mixed model approach to adjust for body-mass index and for the individual macronutrient components of each diet.

No differences in cycle length, body-mass index, or body weight between the two diets were detected. On average, the soy diet contained more carbohydrate, less protein, and less fiber than the usual diets. Serum daidzein and genistein concentrations were at the limits of detection of the assays, but daidzein concentrations were slightly higher during the soy-feeding month than the usual-diet month $(0.15 \pm 0.02$ [SEM] $\mu \mathrm{g} / \mathrm{mL}$ compared to $0.08 \pm 0.05 \mu \mathrm{g} / \mathrm{mL}, P=0.02$, paired $t$-test). Mean serum genistein was not shown to differ between the two feeding conditions. Serum 17ß-estradiol concentrations (AUC, mean, peak) were lower on the soy diet during the follicular phase, the luteal phase, and over the whole cycle. The uncorrected decrease in serum $17 \beta$-estradiol concentrations was about $20 \%$. The statistical significance of the follicular-phase difference was lost when corrected for each macronutrient in the diets. The statistical significance of the luteal-phase difference in $17 \beta$-estradiol was lost when corrected for dietary fiber. Luteal-phase progesterone was lower on the soy diet than the usual diet. The uncorrected decrease in progesterone concentrations was $\sim 30 \%$ on the soy diet. During both diets, individual serum progesterone concentrations were correlated with total non-soy-protein intake, and adjustment for protein intake eliminated the statistical difference in luteal-phase progesterone between the diet conditions. No soy diet-related alterations in serum concentrations of $\mathrm{LH}, \mathrm{FSH}$, and sex hormone-binding globulin were detected.

The study authors indicated that the effect of the low-isoflavone soy diet on menstrual cycle hormones were similar to hormone changes from an isoflavone-containing diet described by them in a previous study (Lu et al., 1996). They concluded that the changes in 17ß-estradiol and progesterone associated with soy diets were likely to be due to soy components other than isoflavones. They also concluded that alterations in gonadotropins and sex hormone-binding globulin did not explain the steroid hormone alterations. They noted the importance of macronutrients on sex steroid concentrations and postulated that differences between studies in hormonal response to dietary soy might be explained by differences in the macronutrient composition of the diets being studied.

Strengths/Weaknesses: The evaluation of non-isoflavone components of soy, the longitudinal study design, the clear inclusion and exclusion criteria, the control for daily energy and nutritional intake, the supervision of meals, and consideration of effect modifiers and confounders are strengths. The sample size was very small, making it difficult to adjust for confounders, and the age range was very wide. There seems to be substantial similarity to the previous study (Lu et al., 2000a), with minimal new information.

Utility (Adequacy) for CERHR Evaluation Process: This study is useful for the evaluation process.

Wu et al. (2000), supported by the California Breast Cancer Research Program, the American Cancer Society, the Susan G. Komen Breast Cancer Foundation, and the Whittier Foundation, evaluated the effects of soy foods on menstrual cycle parameters in 20 employees of the University of Southern California. The subjects ranged in age from 21-44 years. Half of the subjects were Asian. Subjects were evaluated during a 2-cycle baseline period, a 3-cycle intervention period, and a 2- or 3-cycle recovery period. Each cycle went from the first day of a menstrual period until the first day of the next menstrual period. During the intervention period, subjects supplemented their usual diets with tofu, soy milk, and soybeans in any desired combination to provide approximately the same isoflavone content. Dietary intake of soy foods was evaluated with a daily $\log$, and 24-hr diet recall questionnaires were completed approximately weekly. Daily urine collections, which included overnight voids and the first morning voids, were used for measurement of LH, creatinine, and isoflavones. Daily isoflavone 
excretion was estimated based on an assumed daily creatinine excretion rate of $1.2 \mathrm{~g} / \mathrm{day}$. Blood samples were collected on two occasions during each cycle (during cycle Days 10-12 and cycle Days 20-22) and were timed with respect to ovulation based on the urinary LH/creatinine peak. Serum was analyzed for $17 \beta$-estradiol, progesterone, and sex hormone-binding globulin. The effect of the soy diet intervention on serum hormone concentrations were evaluated with $t$-tests and ANOVA.

No significant changes in waist or hip circumference or in body-mass index were detected over the course of the study. There were no changes in the percentage of calories derived from fat, protein, or carbohydrate, cholesterol content, or fiber content over the diet periods. Mean \pm SD isoflavone intake was $32.0 \pm 10.5 \mathrm{mg} /$ day $(36.2 \pm 12.0 \mathrm{mg} /$ day in Asian women and $27.7 \pm 7.0 \mathrm{mg} /$ day in non-Asian women, $P=0.07)$. Urinary isoflavones increased during the soy diet period, more so in Asian women $(32.1 \mu \mathrm{mol} / \mathrm{day})$ than in non-Asian women $(12.8 \mu \mathrm{mol} / \mathrm{day})$. No changes in menstrual cycle length or in lengths of the follicular and luteal phases were detected during the study. Luteal phase serum $17 \beta$-estradiol concentration was decreased $9.3 \%$ during the soy diet period compared to the baseline period. No significant diet-related changes in other hormone or sex hormone-binding globulin determinations were detected. In non-Asian women, no significant changes in $17 \beta$-estradiol concentrations were detected, but sex hormone-binding globulin significantly increased during the follicular and luteal phases by 16-18\%. The authors concluded that soy foods may decrease breast cancer risk by decreasing ovarian production of estrogen.

Strengths/Weaknesses: Strengths are the use of pre-and post-intervention measures and use of multiple measures of compliance with the dietary intervention. Urine was collected daily and good assays were used for hormonal measurements. The use of urinary LH to time ovulation is another strength. Weaknesses are the inclusion of only 20 subjects and the wide age range. The inferences regarding Asian women are hard to support with 10 subjects who had varying baseline histories. The statistical methods seem not to have included repeated measures analysis. The conclusions about breast cancer risk are a stretch inasmuch as no breast cancer-related endpoints were measured.

Utility (Adequacy) for CERHR Evaluation Process: This report is somewhat useful in the evaluation process.

Jakes et al. (2001), supported by the Singapore National Medical Research Council, conducted a study to determine if dietary soy intake was associated with menstrual cycle length in an "uncontrolled community setting." Premenopausal women were recruited from a local hospital $(\mathrm{n}=85)$ and a nursing school $(\mathrm{n}=115)$. Women were menstruating regularly, had no condition that could affect their menstrual cycle or hormone levels, and were not taking medications that could affect hormone levels. The subjects were questioned about soybean intake, menstrual cycle parameters, pregnancies, and use of hormonal drugs. The subjects completed diaries for three consecutive menstrual cycles after the interview. Blood samples were taken at the time of the interviews and analyzed for levels of estrone, 17 $\beta$-estradiol, estriol, LH, and FSH. The subjects' cycle phase at the time of the blood sample was approximated, assuming ovulation 14 days before the start date of the next menstrual cycle. Association between soy intake and cycle length was estimated first by correlation coefficients and then by proportional odds modeling adjusted for age and subject source to account for cycle length being ordered but discrete. Association between soy intake and hormone levels was estimated using correlation coefficients, stratified by cycle phase at the time of sampling.

Exclusions after interviews left 184 subjects in the study, although 12 of these subjects could not be used in analyses using diary information. Estrone was the only hormone found to be significantly correlated with soy-protein intake, with a correlation coefficient of $0.2221(P<$ 
$0.05)$. The researchers found a significant correlation between soy-protein intake and selfreported cycle length, with a correlation coefficient of $0.1728(P<0.05)$, but the correlation with diary-recorded cycle length was not significant. A soy-protein intake higher than the median value of $5.7 \mathrm{~g} /$ day was found to be significantly associated with a longer diary-recorded cycle length $(P=0.034)$ according to unadjusted OR. When adjusted for age and parity, this association was no longer statistically significant $(P=0.06)$.

The study authors concluded that they had shown a likely association between soy-protein intake and menstrual cycle length. They noted that subjects in the highest quartile of soy intake had cycle lengths that were 1-2 days longer that those in the lowest quartile of soy intake. This difference would result in 20 fewer cycles over a lifetime for those women in the highest quartile, and thus, a reduction in lifetime ovarian activity. The authors suggested that reduced breast cancer risk could be explained by a reduction in lifetime ovarian activity and with highsoy intake.

Strengths/Weaknesses: This study was relatively large, although the heterogeneous sources of the sample are a weakness of the study. There were clear inclusion and exclusion criteria, and the menstrual cycle data were of good quality. Sample size and power information were provided, and there was control for age and parity, but no mention was made of modeling to examine multiple other potential confounders. The food frequency questionnaire may not have been an accurate measure of soy intake. Other weaknesses include a lack of information on time since recent oral contraceptive use and collection of only one blood sample per subject.

Utility (Adequacy) for CERHR Evaluation Process: This study is useful for the evaluation process.

Maskarinec et al. (2004a), supported by the National Cancer Institute, evaluated the effect of soy foods on sex hormone levels in premenopausal women during a 2-year intervention. The subjects included 220 regularly cycling women who did not typically have a high intake of soy foods. The women, who ranged from 35-47 years old, were randomized by age and ethnicity to a control group $(\mathrm{n}=111)$ or a group assigned to consume two servings of soy food/day ( $\mathrm{n}$ $=109 ; 1$ serving $\approx 25 \mathrm{mg}$ isoflavones). Subjects were given a choice of tofu, soy milk, roasted soy nuts, soy bars, and soy protein powder. Soy foods were provided without charge. Compliance was monitored with food-frequency questionnaires, soy-food logbooks, random 24-hr dietary recalls, and periodic measurement of urinary isoflavones. At baseline, 3, 6, 12, and 24 months, blood was sampled 5 days after an ovulation predictor kit identified a urinary LH surge. Serum was assayed for $17 \beta$-estradiol, estrone, androstenedione, progesterone, and sex hormone-binding globulin. Statistical analysis used a mixed general linear model that accounted for repeated measures.

During the soy-food intervention, self-reported isoflavone intake was estimated to have increased from a mean $\pm \mathrm{SD}$ of $4.3 \pm 4.9$ to $59.0 \pm 24.5 \mathrm{mg} / \mathrm{day}$ with an increase in urinary isoflavone excretion from $9.2 \pm 20.0$ to $64.1 \pm 67.8 \mathrm{nmol} / \mathrm{mg}$ creatinine. The control group did not alter its consumption of soy foods, as estimated by 24 -hr recall data and urinary isoflavones. No diet-related changes in menstrual cycle length, serum sex hormone-binding globulin, or serum hormone measurements were detected. The authors concluded that if soy foods decrease breast cancer risk, the mechanism is unlikely to involve decreased circulating estrogen levels.

Strengths/Weaknesses: This study had a reasonable sample size. Additional strengths included the randomized intervention over a long term and biologic measures of compliance with the treatment regimen. The report contained good information on recruitment and dropouts. The hormonal assays were appropriate, although there were many missing measurements and the study would have been strengthened by more frequent hormone 
measurement. The unique intervention of adding soy foods to the diets of free-living women is a strength. Weaknesses include the wide range of subject ages and the lack of inclusion of young women in the trial. In addition, despite randomization, substantial differences in soy intake in early life were noted between intervention and control groups.

Utility (Adequacy) for CERHR Evaluation Process: This study is useful in the evaluation process.

4.1.2 Male-Four studies with reproductive endpoints in men consuming soy are presented below in order of publication.

Habito et al. (2000), supported by the Australian Commonwealth Department of Veterans Affairs, evaluated the effects of replacing meat with soy protein on serum sex hormone concentrations. The subjects were 42 omnivorous men between the ages of 35-62 years who were assigned in random order to a 4-week period of eating meat $150 \mathrm{~g} / \mathrm{day}$ and a 4 -week period of eating tofu $290 \mathrm{~g} /$ day ( $35 \mathrm{~g} /$ day soy protein, provided as prepared meals and biscuits). A 2-week period of usual diet served as a washout between the two test diets. The tofucontaining diet was supplemented with butter and lard to make the fat content similar between the two experimental diets. Both diets were equivalent in energy and macronutrients, and differed only by the protein source. Blood was collected before the study and on two occasions 3 days apart at the end of each diet intervention. Serum was assayed using commercial RIA kits for total testosterone, $17 \beta$-estradiol, dihydrotestosterone, and androstanediol glucuronide. Sex hormone-binding globulin was measured using an immunoradiometric assay. Values for the two end-of-diet samples were not different and were averaged. Statistical analyses were carried out using the paired $t$-test or Wilcoxon signed-rank test. Because weight change correlated with sex hormone-binding globulin (and subjects were an average of $400 \mathrm{~g}$ heavier after the tofu diet than after the meat diet), multiple linear regression was used to adjust for weight.

Mean creatinine-adjusted urinary excretion of genistein and daidzein increased 16-20-fold on the tofu diet compared to the meat diet. No significant differences between the diet conditions in mean serum total testosterone, dihydrotestosterone, androstanediol glucuronide, or 17ßestradiol were detected. Weight-adjusted sex hormone-binding globulin was $9 \%$ higher on the tofu diet $(P=0.01)$, and the weight-adjusted testosterone to $17 \beta$-estradiol ratio was $12 \%$ lower on the tofu diet $(P=0.049)$. The authors concluded that overall androgenic activity may be reduced by dietary isoflavones, which increase sex hormone-binding globulin with a consequent reduction in free testosterone. They indicated that testosterone is more avidly bound to sex hormonebinding globulin than is $17 \beta$-estradiol, and a reduction in the binding globulin would produce a greater decrease in testosterone than in $17 \beta$-estradiol activity. The authors cautioned that the biologic significance of the small change identified with short-term dietary soy was uncertain, and that the effects of a soy-containing diet over many years required additional study.

Strengths/Weaknesses: The randomized cross-over design with subjects serving as their own controls is a strength. There was a good washout period before study participation, and total dietary intake was controlled. The authors provided sample size and power calculations. Weaknesses include the wide age range of the subjects, the small sample, the lack of adjustment for body-mass index or other potential confounders, and the inadequate assessment of compliance with diet.

Utility (Adequacy) for CERHR Evaluation Process: This study is useful for the evaluation process. 
Nagata et al. (2000), supported by Gifu University and the Second-Term Comprehensive Strategy for Cancer Control in Japan, evaluated the association between estimated dietary-soy consumption and serum androgen and estrogen levels in Japanese men. Study subjects were recruited from participants in a larger study on the association between diet and cancer. There were 256 men selected randomly to be invited to participate in the hormone-level study; of these men, 97 agreed to participate. Blood was collected from participants, and serum was separated and frozen until analysis by RIA for 17ß-estradiol, estrone, total and free testosterone, dihy-drotestosterone, and sex hormone-binding globulin. Information on diet, smoking, ethanol use, exercise, and medical history was obtained by questionnaire. Isoflavone intake from soy was estimated from a daily food-frequency questionnaire, administered 12 times over the course of a month, about 3 years before the collection of blood samples. [It is not clear if all participants completed a food frequency questionnaire 12 times or this description applies to a smaller group used in a reproducibility sub-study.] The association between soy intake and serum hormone concentrations was evaluated using Spearman rank correlation coefficients after regressing hormone and soy-intake values on confounders including age, body-mass index, smoking status, and ethanol. Estimated dietary intake of fat, cholesterol, carbohydrate, and vitamins $\mathrm{B}_{1}$ and $\mathrm{D}$ were significantly correlated with serum androgens and were included in the regression model for serum androgens. The men in the study were a mean \pm SD of $60.5 \pm 10.7$ years of age with a mean body-mass index of $22.6 \pm 3.0 \mathrm{~kg} / \mathrm{m}^{2}$. Serum $17 \beta$ estradiol was inversely correlated with soy-product intake and with isoflavone intake ( $r=$ $-0.32 ; P<0.01$ in the fully adjusted model). None of the other hormone measurements showed a significant correlation with either soy-product intake or isoflavone intake. The authors proposed that soy isoflavones may inhibit enzymes such as aromatase that are involved in estrogen biosynthesis.

Strengths/Weaknesses: The random selection of subjects invited to participate is a strength of this study. Information on exposure was good, with multiple assessments of dietary intake. The sample was relatively large. The control for some potential confounders is an additional strength. Weaknesses include the wide age range, although adjustment was carried out, and the collection of dietary data long before blood sampling.

Utility (Adequacy) for CERHR Evaluation Process: This study is useful for the evaluation process.

Mitchell et al. (2001), supported by U.K. government agencies, evaluated the effects of a soyextract food supplement on reproductive parameters in men. Healthy 18-35-year-old nonvegetarian men were given a daily 500-mg tablet of a commercial preparation called Regent ${ }^{\mathrm{TM}}$, supplied by its manufacturer, Novogen Ltd, Australia. This supplement contained $40 \mathrm{mg}$ total isoflavones (genistein, daidzein, and glycitein). Men were asked to take one tablet each day for 2 months. Assessments were carried out monthly, including two assessments before the supplement, two assessments while on the supplement, and three assessments after stopping the supplement. Assessments included an evaluation of testis size, blood sampling for measurement of genistein, daidzein, $17 \beta$-estradiol, testosterone, FSH, and LH, and semen analysis including the use of computer-assisted sperm analysis (CASA). Fifteen men were initially enrolled; one subject discontinued himself from the study midway through and was excluded from analysis, and three subjects did not appear for the final assessment. Statistical analysis was by ANOVA with adjustment for the covariates alcohol, smoking, technician, season, and sexual abstinence.

Mean plasma genistein peaked in the first month of supplement use at approximately $1 \mu \mathrm{M}$ [270 ng/mL aglycone equivalent], and mean plasma daidzein peaked at about $0.5 \mu \mathrm{M}$ [127 $\mathbf{n g} / \mathbf{m L}$ aglycone equivalent]. There were no significant treatment effects on testicular volume, plasma hormone determinations, or semen analysis parameters. [Percent of morphologically 
normal motile sperm seemed to decrease over the course of the study; however, the authors indicated that this apparent effect was attributable to changes in evaluation technique that were introduced during the study period.] The authors concluded that their study did not identify adverse male reproductive effects at this dose of phytoestrogens but could not exclude an adverse effect of higher intake levels.

Strengths/Weaknesses: The use of younger men, exposure to a standardized tablet, collection of serial blood samples, use of subjects as their own controls, and adjustment for potential confounders are strengths of this study. The authors provided sample size and power calculations. Weaknesses include the small sample and high drop-out rate, which makes multivariate analysis difficult. It is unclear whether the hormone data were normally distributed and, if not, whether the data were transformed before analysis. There was no measure of compliance with the assigned regimen. There was only one dose level, precluding doseresponse assessment, and the exposure was short-term. It seems that semen was inconsistently evaluated over the course of the study.

Utility (Adequacy) for CERHR Evaluation Process: This study is of minimal utility for the evaluation process.

Nagata et al. (2001), supported by the Japanese Ministry of Health and Welfare, examined the effects of soy intake on serum androgen and estrogen levels in healthy men who were either students or teachers at a nursing school. Subjects were randomly assigned to a soy-milk group $(\mathrm{n}=17)$ or control group $(\mathrm{n}=17)$. During an 8-week period, subjects in the soy-milk group were instructed to drink $400 \mathrm{~mL} /$ day soy milk that was supplied to them and no other soy milk. Men from both groups were instructed to continue eating their regular diets and were asked to keep dietary records, which were used to estimate intake of soy products, macronutrients, micronutrients, and isoflavones. Blood was collected just before the dietary phase and then every 2 weeks for 12 weeks. Serum was separated for measurement of estrogens and androgens by RIA. Statistical analyses included standard and paired $t$-tests for dietary parameters. Longitudinal hormone data were analyzed using a mixed linear regression model.

Compared to baseline levels, increased intake of vitamin B1, iron, and phosphate and decreased intake of retinol, vitamin A, and salt were observed in the soy-milk group over the course of the study. No significant changes in nutrient intake were noted in the control subjects over the course of the study. Mean intake of soy milk throughout the study was estimated at $360 \mathrm{~mL}$. Mean isoflavone intake from soy milk and other sources in the soy-milk group increased from $\sim 16 \mathrm{mg}$ before dietary intervention to $\sim 93 \mathrm{mg}$ at the end of the dietary period. [Values are assumed to represent daily intakes.] In the control group, mean isoflavone intake was $\sim 18$ $\mathrm{mg}$, similar at the start and end of the study. No significant differences in serum levels of $17 \beta$-estradiol, total and free testosterone, or sex hormone-binding globulin were detected between the soy-milk and control groups. A significant difference between the soy-milk and control groups were observed for serum estrone, which tended to decrease in the soy-milk group and increase in the control group. The study authors concluded that soy-milk intake by men may result in modified circulating estrone concentration.

Strengths/Weaknesses: The use of men as their own controls, serial blood collections, randomized assignment, information on doses, sample size calculations, and use of transformed hormone data are strengths of this study. Weaknesses include the small sample size, the wide age range, and the lack of measures of compliance with the assigned regimen. Although the authors adjusted for some potential confounders in multivariate analysis, the small sample size makes it difficult to adjust effectively. The use of only Japanese men may decrease the generalizability of the results. 
Utility (Adequacy) for CERHR Evaluation Process: This study is somewhat useful for the evaluation process.

\subsection{Experimental Animal}

4.2.1 Female-Boettger-Tong et al. (1998), supported by NIH and the John P. McGovern Foundation, described observations of possible effects of animal feed on the reproductive system of the female Sprague-Dawley rat. [This report provided limited information; e.g., there was no information on animal numbers, experimental procedures, quantitative results, or statistical analysis.] A series of experiments in ovariectomized, 30-day-old rats showed little response to injected estradiol [not otherwise specified] as measured by induction of marker genes such as $c$-fos. It seemed that the lack of response was due to unusually high levels of marker gene expression in control rats. To investigate possible estrogenic activity from exposure to diet or other environmental factors, uterine and vaginal sections were prepared and examined from 30-day-old ovariectomized rats kept at the laboratory in 1995 but not treated with hormones. The sections were compared to those from rats kept at the laboratory 2 years earlier (1993), a time when there were low basal levels of gene expression and robust responses to hormonal treatments. Compared to the rats from 1993, the rats from 1995 displayed an estrogenic response, as indicated by thicker vaginal epithelium, increased height of uterine luminal epithelium cells, and a more edematous uterine stroma. Some signs of estrogenicity were observed in 20-day-old rats obtained from the same vendor, which used diet from the same supplier as the study authors used. No signs of estrogenic response were seen in 20-day-old rats obtained from an alternate vendor. Studies were conducted with rats from the alternate vendor, and it was determined that the laboratory diet, consisting primarily of soy and alfalfa, was the most likely source of the unexpected estrogenic response. Analysis of feed by GC-MS showed genistein at $21 \mathrm{mg} / 100 \mathrm{~g}$ feed and daidzein at $14 \mathrm{mg} / 100 \mathrm{~g}$ feed. [The report abstract states that the level of daidzein was $14 \mathrm{mg} / \mathrm{kg}$ feed, which would be equivalent to $1.4 \mathrm{mg} / 100 \mathrm{~g}$ feed. Using USEPA (1988) assumptions for weanling rat body weight and food intake, CERHR estimated intake rates of $31 \mathrm{mg} / \mathrm{kg}$ bw/day genistein and $21 \mathrm{mg} / \mathrm{kg} \mathrm{bw} / \mathrm{day}$ daidzein. Daidzein intake would be $2.1 \mathrm{mg} / \mathrm{kg}$ bw/day if the number in the abstract is correct.] Levels of other isoflavones were not analyzed, but the mycoestrogens zearalenone and zearalenol were not detected by a contract laboratory. In earlier study reports, genistein levels were measured in rat feed at $6.8 \mathrm{mg} / 100 \mathrm{~g}$ feed, and daidzein was measured at $0.6 \mathrm{mg} / 100 \mathrm{~g}$ feed. The diet vendor was notified and sent a sample of the feed from the batch in question. After testing of the feed in an estrogen-sensitive cell culture, the vendor concluded, "It appears that a component of the...rat/mouse diet which was used at your facility in the summer of 1995 had a high level of estrogen." To further examine the effects of diet, 20-dayold rats were obtained from the alternate vendor, ovariectomized, and fed either the diet in question or the alternate diet with the same general composition. At 30 days of age, the uteri from animals fed the diet in question were larger and more vascularized than uteri from animals receiving the alternate diet. The study authors concluded that it is necessary to use diets with controlled ingredients and formulations and to monitor the diets for contaminants.

Strengths/Weaknesses: This observation is important for researchers working on estrogenic effects. Strengths are that genistein and daidzein were measured in the actual feed given to the animals, and that the feed was shown to have baseline effects on the rat uterus; however, it is a weakness that uterine weight was not used as an endpoint. A methods section was not provided, and no information was given on the number of animals used. Use of 17ß-estradiol as a positive control would have strengthened the study.

Utility (Adequacy) for CERHR Evaluation Process: This report is useful in showing that dietary soy can have estrogenic effects in rodents, but it is not useful in the evaluation process. 
Gallo et al. (1999), support not indicated, carried out a reproduction study of dietary soy in female Wistar rats. The control diet was a commercial soy-containing diet. [The soybean meal in the diet was solvent-extracted; isoflavone content was not reported.] Modified diets were made by adding a soy extract that contained $12 \%$ isoflavones. The supplement was added at dietary levels of $0.7,1.2$, and $2.4 \%$. Rats were randomized to one of the diet groups at weaning $(n=20 /$ group $)$. The day of vaginal opening was recorded, and daily vaginal smears were examined for estrous cycle stage on PND 63-77. Beginning on PND 77, females were paired with untreated males, and the day of vaginal sperm or a vaginal plug was considered GD 0. Dams were allowed to litter (PND 0). Pups were sexed and examined for external abnormalities on PND 1. Dams and pups were killed on PND 7, and pups were again examined for external abnormalities. Dams were necropsied with histologic examination of ovaries, uterus, and vagina. Data were analyzed using ANOVA with post-hoc Dunnett multiple comparison test or by the Kruskal-Wallis test followed by the Dunn multiple comparison test.

Results are summarized in Table 45. Unscheduled deaths or litter loss occurred in all treatment groups and were not considered treatment-related. Growth suppression was noted and at least partially attributed to reduced feed consumption [data not shown]. Despite soy-associated alterations in age at vaginal opening and estrous cycle length, adverse effects on reproductive function were not detected. There was no detected effect of treatment on external abnormalities of pups.

The authors concluded that dietary soy had estrogenic effects on the female reproductive system, either as a direct consequence of phytoestrogen exposure or through effects on ovarian follicles. They indicated that toxicity occurred with a daily isoflavone intake that exceeded human dietary isoflavone intake by more than 100 -fold, a fact they recommended considering in interpretation of the results of this study. [Measurements of isoflavone or feed intakes were not provided. Assuming a female rat ate $0.08 \mathrm{~kg}$ feed $/ \mathrm{kg}$ bw/day (USEPA, 1988) and that the middle- and high-dose diets contained isoflavones 0.14 and $0.29 \mathrm{~g} / 100 \mathrm{~g}$ feed, daily isoflavone intake would have been $64 \mathrm{mg} / \mathrm{kg}$ bw at the low dose, $112 \mathrm{mg} / \mathrm{kg} \mathrm{bw/day}$ at the middle dose, and $232 \mathrm{mg} / \mathrm{kg}$ bw at the high dose. The authors cite an estimate of human isoflavone consumption in Japan as $3 \mathrm{mg} / \mathrm{kg}$ bw/day.]

Strengths/Weaknesses: The use of three levels of dietary isoflavones and the examination of female reproductive organ morphology are strengths. Weaknesses include the lack of information on the isoflavone levels in the chow, the failure to determine isoflavone intake, and the lack of detailed information on the offspring. The isoflavone intakes seem to be much higher than adult human exposure levels. The treatment duration from weaning through adulthood makes it difficult to interpret whether alterations would have been developmental, reproductive, or a combination of the two.

Utility (Adequacy) for CERHR Evaluation Process: This study is useful in evaluating the effects of high-dose soy isoflavones on the female reproductive tract and in showing an apparent lack of effect on reproductive function.

Lund et al. (2001a), supported by the National Science Foundation and Brigham Young University, conducted a series of experiments to examine reproductive and developmental effects in rats fed phytoestrogen-containing feed. From 50 days of age through completion of the study, five female Long-Evans rats per group were fed a soy-based diet containing $600 \mu \mathrm{g}$ phytoestrogens/g diet (phyto-600) or a fish meal-based diet that was phytoestrogen free.

[Assuming a female rat ate $0.08 \mathrm{~kg}$ feed/ $/ \mathrm{kg}$ bw/day (USEPA, 1988), isoflavone intake in the phyto-600 group would have been $48 \mathrm{mg} / \mathrm{kg}$ bw/day.] Diets were similar in protein, fat, amino acid, mineral, and vitamin content. From $80-85$ days of age, the rats were mated with males fed the same diet. The rats were allowed to deliver and nurse their offspring, which were 
used in experiments described in Section 3.2. When $\mathrm{F}_{0}$ rats were 150-163 days of age, vaginal cytology was monitored for 14 days. Length and regularity of estrous cycles were normal in rats fed either diet [data were not shown]. The authors' conclusions were focused on the developmental effects of the phytoestrogen-containing diet, which are discussed in Section 3.2.

Strengths/Weaknesses: Use of a well-defined high-phytoestrogen diet, evaluation of multiple endpoints, and use of a two-generation design are strengths. The measurement of phytoestrogens in serum and demonstration of relevance of these levels to human exposures is helpful. The comparison to a fish-meal diet makes interpretation difficult inasmuch as these diets are likely to differ with respect to many components other than phytoestrogens.

Utility (Adequacy) for CERHR Evaluation Process: The observation that the volume of the SDN-POA in males was decreased by removal from soy for 40 days during adulthood (discussed in Section 3.2) is intriguing; however, the main focus of this study was developmental effects of dietary soy. The lack of effect of the soy diet on estrous cycles is of use in the evaluation of reproductive effects.

Patisaul et al. (2001), supported by the National Science Foundation and Emory University, evaluated the effects of a soy supplement on sexual function in ovariectomized female Long Evans rats. All animals used in the study had been shown to mate after administration of estradiol benzoate. Female rats were fed a phytoestrogen-free diet to which was added a ground commercial-soy supplement at a concentration of $0 \%$ or $0.35 \%$. Based on previous studies, the authors estimated that the supplemented diet contained genistein $13 \mathrm{ppm}$ and daidzein $33 \mathrm{ppm}$, producing plasma phytoestrogen concentrations between those of humans on a typical Western diet and on an Asian diet. [Neither dietary phytoestrogens nor plasma concentrations were measured in this study, although the phytoestrogen content of the commercial supplement was determined. Assuming a female Long-Evans rat consumes $0.08 \mathrm{~kg}$ feed/ $\mathrm{kg}$ bw/day (USEPA, 1988), mean genistein intake would have been $1.04 \mathrm{mg} / \mathrm{kg}$ bw/day and mean daidzein intake would have been $2.64 \mathrm{mg} / \mathrm{kg} \mathrm{bw/day.]} \mathrm{Some} \mathrm{females} \mathrm{were} \mathrm{given}$ s.c. estradiol benzoate $48 \mathrm{hr}$ before mating, and all females were treated with s.c. progesterone $4-5 \mathrm{hr}$ before mating. There were four treatment conditions ( $\mathrm{n}=8 /$ group) used to evaluate the effects of soy: soy-free diet only, soy diet only, soy-free diet + estradiol benzoate, and soy diet + estradiol benzoate. A s.c. injection of oil vehicle was given to rats not receiving estradiol benzoate. The experimental procedure consisted of placing each female in a mating chamber with an untreated male retired breeder for $30 \mathrm{~min}$. The breeding apparatus included a smaller chamber into which the female could escape. Males were not able to follow the females into the escape chamber due to the size of the tunnel between the two compartments. During a 10min period beginning $5 \mathrm{sec}$ before the first attempted mount, counts were made of the number of attempted mounts by the male and the number of lordosis responses by the female. A lordosis quotient was calculated as the percent of mount attempts resulting in lordosis. Data were analyzed using ANOVA with post-hoc Fisher least significant difference test.

In a second experiment, ovariectomized female rats were divided into four similar diet \pm estrogen groups ( $\mathrm{n}=10$ or 11 /group) except that estrogen was administered using a s.c. silastic capsule containing $17 \beta$-estradiol. Measured $17 \beta$-estradiol concentrations in blood were about four times those found in ovary-intact animals. An empty capsule was implanted in animals not assigned to estrogen treatment. The implants were inserted 2 days after the dietary intervention was started. Four days after implant insertion, blood was collected for determination of plasma 17 $\beta$-estradiol, uteri were removed and weighed, and brains were removed and serial sections were made from the lateral septum to the caudal end of the ventromedial nucleus of the hypothalamus. In situ hybridization was carried out in brain sections using a probe for ER $\beta$ mRNA, and autoradiography was carried out for oxytocin 
receptor, which is upregulated by ER $\alpha$ agonists. Data were analyzed using ANOVA with posthoc Fisher least significant difference test.

Estradiol benzoate treatment resulted in an increase in the lordosis quotient from 6.7\% to 91.8\% in animals fed a phytoestrogen-free diet. The feeding of the soy diet resulted in a significant decrease of the lordosis quotient to $72.8 \%$ in animals also given estradiol benzoate. In the females that were not given estrogen, the number of attempted mounts was decreased at $P$ $<0.06$ by the soy diet from a mean of $\sim 11$ to a mean of $\sim 3$ [estimated from a graph]. In females given estradiol benzoate, no effect of dietary soy on number of attempted mounts was detected. There was no treatment-related difference detected in the amount of time the female remained in the same compartment as the male during the mating trial. The in situ hybridization for $E R \beta$ mRNA showed message in the paraventricular nucleus but not the ventromedial nucleus of the hypothalamus. Estrogen treatment decreased the expression of $E R \beta$ by $41 \%$; this decrease was not affected by exposure to the soy diet. In animals not receiving estrogen, $E R \beta$ expression was increased $27 \%$ by the soy diet. $17 \beta$-Estradiol increased the expression of oxytocin receptor in the ventromedial nucleus. This increase was somewhat attenuated by the soy diet. No effect of the soy diet alone on oxytocin receptor expression was detected. The soy diet was not shown to result in either increased uterine weight or attenuation of the uterine hypertrophy produced by $17 \beta$-estradiol.

The authors concluded that the soy supplement acted as an anti-estrogen on female sexual behavior. They further proposed that the soy-mediated decrease in hypothalamic oxytocin receptor expression might mediate the suppression of sexual behavior.

Strengths/Weaknesses: This well-designed study reported important observations on the antiestrogenic effects of soy phytoestrogens in the brain. The use of several estrogen related endpoints and the measurement of circulating $17 \beta$-estradiol are strengths. The lack of information on the number of animals is a weakness. There was no uterotropic effect of dietary soy, but the use of adult animals may be an explanation.

Utility (Adequacy) for CERHR Evaluation Process: This study is useful for the evaluation process.

Patisaul et al. (2004), supported by NIH, the National Science Foundation, and Emory University, evaluated the effects of a soy supplement on sexual function in ovariectomized female Long-Evans rats. All animals used in the study had been shown to mate after administration of estradiol benzoate. Female rats were fed a phytoestrogen-free diet to which was added a ground commercial soy supplement at a concentration of $0 \%$ or $0.35 \%$. Based on previous studies, the authors estimated that the supplemented diet contained genistein $13 \mathrm{ppm}$ and daidzein $33 \mathrm{ppm}$, producing plasma phytoestrogen concentrations between those of humans on a typical Western diet and those of humans on an Asian diet. [Neither dietary phytoestrogens nor plasma concentrations were measured. Assuming a female LongEvans rat consumes $0.08 \mathrm{~kg}$ feed/kg bw/day (USEPA, 1988), mean genistein intake would have been $1.04 \mathrm{mg} / \mathrm{kg}$ bw/day and mean daidzein intake would have been $2.64 \mathrm{mg} / \mathrm{kg} \mathrm{bw} /$ day. The duration of dietary treatment before testing sexual function was not given.] Half of the females were given s.c. estradiol benzoate $48 \mathrm{hr}$ before mating, and all females were treated with s.c. progesterone $4-5 \mathrm{hr}$ before mating. There were four treatment conditions (n $=6 /$ group) used to evaluate the effects of soy: soy-free diet only, soy diet only, soy-free diet + estradiol benzoate, and soy diet + estradiol benzoate. [Two additional groups were used to evaluate the effects of tamoxifen with or without estradiol benzoate; all tamoxifentreated animals received the phytoestrogen-free diet.] The experimental procedure consisted of placing each female in a mating chamber with an untreated male retired breeder for 10 min. The breeding apparatus included a smaller chamber into which the female could 
escape. Males were tethered and were not able to follow the females into the escape chamber. During the 10-min cohabitation period, counts were made of the number of attempted mounts by the male and the number of lordosis responses by the female. A lordosis quotient was calculated as the percent of mount attempts resulting in lordosis. Females with a lordosis quotient $>50 \%$ were evaluated for the number of escapes and the amount of time spent in the escape chamber before returning to the male after mounting, intromission, or ejaculation. Data were analyzed using ANOVA with post-hoc Fisher least significant difference test.

Estradiol benzoate treatment resulted in an increase in the lordosis quotient from $1 \%$ to $87.9 \%$ in animals fed a phytoestrogen-free diet. The feeding of the soy diet resulted in a significant decrease of the lordosis quotient to $62.7 \%$ in animals also given estradiol benzoate. This decrease was similar to that produced by tamoxifen. Female hopping and darting behavior was also decreased by dietary soy. The percent of sexual contacts that were followed by escape of the female was decreased by dietary soy, but no effect on the latency to return to the male was detected. The authors noted that only four of six estradiol benzoate-treated soy-exposed females received an ejaculation, and only two of these four went into the escape chamber thereafter, limiting the ability to detect a treatment effect. There was no effect of soy supplementation on sexual behaviors in animals that were not given estradiol benzoate.

The authors concluded that the effects of soy supplementation on female sexual behavior are anti-estrogenic.

Strengths/Weaknesses: The use of tamoxifen as a comparator is a strength of this study, which is generally supportive of the first study (Patisaul et al., 2001). The number of animals used was too low for definitive conclusions, however, and the endpoints were somewhat subjective.

Utility (Adequacy) for CERHR Evaluation Process: This study is useful in support of the first study (Patisaul et al., 2001).

Woclawek-Potocka et al. (2005), supported by the Polish Ministry of Scientific Research and Information Technology, evaluated the effect of feeding soybeans on fertility in cows.

Experiment 1 involved 12 cows in Zalesie that were fed a standard diet containing sunflower grain, wheat bran, and rye (phytoestrogen content $<300 \mathrm{mg} / \mathrm{kg}$ feed), and 12 cows in Watkowice that were fed a diet of soybeans, rape, and grain (phytoestrogen content $1900 \mathrm{mg} /$ $\mathrm{kg}$ feed). The animals began their assigned diets after calving in September or October, 2002 and continued the diets until the following year when all animals were inseminated with semen from the same bull. Blood samples were collected every 3 months until October, 2003 and urine was collected in October, 2003. [The date of insemination, number of inseminations, and order of insemination between members of the two herds were not specified. It is not known whether all animals were inseminated with aliquots of the same seminal preparation or with aliquots from different ejaculates. Because the date of the beginning of the observation period was not given, the length of observation after insemination is not known.] In Experiment 2, cows from the Zalesie herd were divided into a group given the standard diet and a group given the soybean diet ( $n=5 /$ group). After 2 weeks, estrous cycles were synchronized using progestin withdrawal, and animals were each inseminated twice with semen from a single bull. Blood was collected about every 3 days for 21 days. In an in vitro experiment, bovine uteri were collected from an abattoir and slices were incubated with daidzein, the daidzein metabolite equol, genistein, or the genistein metabolite 2-ethylphenol at concentrations of $10 \mathrm{nM}$ (determined previously to be optimal), and media were evaluated for concentration of prostaglandins $\mathrm{E}_{2}$ and $\mathrm{F}_{2 \alpha}$. Data were analyzed using $\chi^{2}$ or repeatedmeasures ANOVA with post-hoc Bonferroni test. 
In Experiment 1, the soybean-fed Watkowice cows had higher mean serum concentrations of cholesterol, total protein, and alanine aminotransferase than did cows from the standard-fed Zalesie herd. The pregnancy rate in the standard-fed Zalesie herd (100\%) was significantly greater than that in the soybean-fed Watkowice herd (60\%) [Only animals achieving behavioral estrus were inseminated; the number of these animals in each group was not given.] In Experiment 2, cows from the Zalesie herd were not shown to differ in pregnancy rates by diet (three pregnancies in five soybean-fed cows and four pregnancies in five standardfed cows). Plasma concentrations of a prostaglandin $\mathrm{F}_{2 \alpha}$ metabolite were higher in soybeanfed than standard-fed cows and correlated with blood concentrations of equol and 2ethylphenol. Genistein, daidzein, and their metabolites elevated prostaglandin $\mathrm{E}_{2}$ and $\mathrm{F}_{2 \alpha}$ production by cultured endometrium. The authors concluded that feeding soybeans interferes with fertility and possibly early embryo survival in cows, and proposed that an increase in endometrial production of luteolytic prostaglandins by the endometrium might mediate the antifertility effect of soybean phytoestrogens.

Strengths/Weaknesses: Strengths are the use of cows to evaluate effects of soybeans on pregnancy rate and possibly luteal function, measurement of circulating phytoestrogen concentrations, and determination of circulating prostaglandin metabolite and progesterone concentrations. Weaknesses are the lack of detail on the studies of pregnancy rates, making it unclear whether feeding soybeans reduced the rate, the lack of diet effect on circulating progesterone, suggesting that luteal function was unaffected, and the lack of clarity on the number of animals inseminated in Experiment 1. The evidence for an impact of dietary soybeans on luteal function is quite indirect, consisting of an increase in prostaglandin $\mathrm{F}_{2 \alpha}$ from the uterus.

Utility (Adequacy) for CERHR Evaluation Process: Although the data suggest that there may be reduced pregnancy rates in cows fed a diet containing soybeans, unclear reporting makes this conclusion unreliable. The lack of clear effects on fertility measurements detracts from the utility of this study.

Piotrowska et al. (2006), supported by the Polish Ministry of Scientific Research and Information Technology, evaluated the effect of feeding soybeans on corpus luteum progesterone production in cows. In Experiment 1, five normally cycling cows/group were fed a standard diet consisting of sunflower grain, wheat bran, rye, beet pulp, maize, and hay or a soybean diet containing soybeans, rape seed, wheat grain, beet pulp, molasses, maize, grass, and hay. The authors estimated that the standard diet contained phytoestrogens at $<300 \mathrm{mg} / \mathrm{kg}$ feed and the soybean diet contained phytoestrogens at $\sim 1900 \mathrm{mg} / \mathrm{kg}$ feed. Jugular venous blood samples were taken on estrous cycle days $0,3,6,9,12,15$, and 21 for measurement of progesterone and the isoflavone metabolites equol and $p$-ethylphenol. In Experiment 2, four cows/group were fed the standard or soybean diet and corpora lutea were obtained on Day 8 of the estrous cycle. The corpora lutea were assayed for progesterone, equol, and $p$-ethylphenol. Corpus luteum tissue was cultured for $18 \mathrm{hr}$ in the presence of $\mathrm{LH}$, prostaglandin $\mathrm{E}_{2}$, or prostaglandin $\mathrm{F}_{2 \alpha}$, and progesterone was measured in media. [A third experiment involving the perfusion of corpus luteum tissue with equol or $p$-ethylphenol will not be discussed here.] Data were analyzed using repeated measures ANOVA followed by Bonferroni multiple comparison test. Data from Experiment 2 were normalized for tissue weight.

Plasma equol and $p$-ethylphenol were almost undetectable in animals fed the standard diet but peaked on estrous Day 3 in animals on the soybean diet. No effect of diet on estrous cycle length was detected, but serum progesterone was significantly reduced by the soybean diet on cycle Days 15 and 18. [The largest decrease was by $\mathbf{5 0 \%}$ on cycle Day 18, estimated from a graph.] Equol and $p$-ethylphenol were higher in corpora lutea of soybean-fed cows than standard-fed cows and progesterone was lower [by $45 \%$, estimated from a graph]. Corpora 
lutea from soybean-fed cows had a decreased response to $\mathrm{LH}$, prostaglandin $\mathrm{E}_{2}$, and prostaglandin $\mathrm{F}_{2 \alpha}$ compared to corpora lutea from standard-fed cows. [Progesterone was decreased 39-47\%, estimated from a graph.] The authors concluded that dietary soybeans may alter corpus luteum function in cows and result in lower rates of insemination or higher rates of early embryo loss.

Strengths/Weaknesses: Use of cows to evaluate the effect of soy phytoestrogens on luteal function is a strength and the evidence for effects on luteal progesterone production is strong. There is, however, limited information on the effect of the soybean diet on actual pregnancy outcomes. The study cited a previous study (Woclawek-Potocka et al., 2005) purporting to show a decrease in pregnancy rates in soybean-fed cows. Data presentation in that previous study was not strong, and fertility results seemed equivocal. There seemed to be confusion about the role of prostaglandin $\mathrm{F}_{2 \alpha}$ on luteal progesterone secretion. Prostaglandin $\mathrm{F}_{2 \alpha}$ is thought to be luteolytic and prostaglandin $\mathrm{E}_{2}$ luteotropic in cows, but both prostaglandins stimulated progesterone secretion from luteal tissue in this study. Attempts to reconcile this contradiction were weak.

Utility (Adequacy) for CERHR Evaluation Process: The in vitro data support the hypothesis that soy phytoestrogens reduce luteal function and, as a result, reduce fertility in cows; however, whether progesterone would be sufficiently reduced to impair fertility is not known, and more compelling data on pregnancy rates are needed before this study can be considered useful in the evaluation process.

Wood et al. (2006), supported by NIH, fed adult female cynomolgus monkeys a soy protein $\operatorname{diet}(\mathrm{n}=27)$ or a casein-lactalbumin diet $(\mathrm{n}=25)$. The diets were similar except for the protein source. The soy protein diet contained isoflavones $1.88 \mathrm{mg} / \mathrm{g}$ protein, which the authors estimated as equivalent to human consumption of genistein $91 \mathrm{mg}$, daidzein $31 \mathrm{mg}$, and glycitein $7 \mathrm{mg}$ for an $1800 \mathrm{kcal}$ diet. After a 6-month baseline period on an isoflavone-free control diet, the test diets were given for 12 months. Animals were fed in social groups and individual intakes were not measured. Daily vaginal swabs were obtained. [One part of the Methods section indicates that daily swabs were obtained "for the 6 months surrounding baseline and treatment evaluations," and another part of the Methods section says that swabs were taken "before and after each treatment phase." It is not clear when these swabs were taken. Inasmuch as the animals were sedated for the taking of vaginal swabs, it is possible that the animals under-went prolonged periods of daily sedation.] Serum progesterone was measured 3 times/week for 2 months during the baseline and the treatment periods. On cycle Days 11 and 22 at the end of the baseline period and cycles Days 5 and 22 at the end of the treatment period, blood was collected for measurement of serum $17 \beta$-estradiol, progesterone, and isoflavones, uterine ultrasound was carried out, and breast biopsies were obtained. [Details of the breast biopsy evaluations addressed possible cancer risk in adults fed soybeans and will not be addressed here.] Data were analyzed using a mixed general linear model.

After 10 months on the soy protein diet, mean \pm SEM serum isoflavone concentrations were said to be comparable to values reported in humans (equol 731.3 $\pm 59.9 \mathrm{nM}[\mathbf{1 7 7} \pm \mathbf{1 5} \boldsymbol{\mu g} / \mathbf{L}]$,

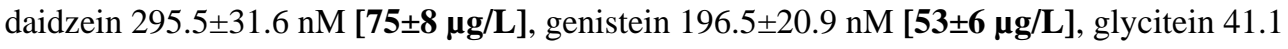

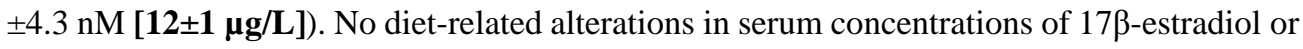
progesterone [pooled across cycle phases], menstrual cycle length, uterine area, or vaginal maturation were detected. The authors' conclusions were restricted to comments about breast cancer risk.

Strengths/Weaknesses: This experiment was well-designed and executed. There were a sufficient number of animals, and serum hormone measurements utilized standard assays. The 
lack of clarity on the timing of vaginal swabs is a weakness but does not detract from the overall utility of the study.

Utility (Adequacy) for CERHR Evaluation Process: These findings are useful in showing that dietary levels of soy equivalent to those consumed by humans had no detectable effect on several estrogen-dependent end-points in cynomolgus monkeys.

4.2.2 Male-Male reproductive studies of dietary soy or soy isoflavones have been carried out in rodents. Studies on rats are presented first followed by a study on mice.

Sharma et al. (1992), supported by NIH, the Finnish Cancer Foundation, and the Medical Research Council of the Academy of Finland, examined the effects of soy diet on the rat prostate. For 11 weeks, five or six adult male Sprague-Dawley rats/group were fed one of three diets: a commercial rat chow with soy as the protein source (control group), a specialty soyfree diet with casein as the protein source, or a specialty diet with soy as the protein source. The two specialty diets contained identical ingredients, with the exception of the protein source. At the end of the treatment period, rats were killed and prostates were removed, weighed, and examined histologically. Phytoestrogens and metabolites were measured in urine by GC-MS. Data were analyzed by ANOVA followed by Duncan multiple range test. Urinary excretion of genistein, daidzein, and their metabolites was approximately equal in the control and soydiet groups, but much lower in the soy-free diet group. No significant effects on ventral, lateral, and dorsal prostate weights between diet groups were detected. Morphology of ventral and dorsal prostate was not observed to be affected by diet. Severity and incidence of prostatitis was increased in the lateral prostate of rats fed the soy-free diet compared to rats fed the soy or control diets. Incidence of prostatitis was 16\% in rats fed the control diet, $80 \%$ in rats fed the soy-free diet, and $0 \%$ in rats fed the soy diet. The study authors concluded that their results suggest that dietary soy protects against development of prostatitis in rats.

Strengths/Weaknesses: It is difficult to reconcile the lack of effect of the different diets on weight of different prostate lobe with the large difference in reported rates of prostatitis. Presumably, the term "prostatitis" refers to a unique inflammatory process in the lateral lobe. There is no evidence that this condition is relevant to human prostatitis, which is due to bacterial infection.

Utility (Adequacy) for CERHR Evaluation Process: This study is not useful for the evaluation process.

Weber et al. (1999), supported by the National Science Foundation, NIH, and Brigham Young University, evaluated the effects of dietary soy on plasma testoster-one and brain concentrations of steroid-metabolizing enzymes in male Sprague-Dawley rats. The animals were given standard laboratory chow until they were 74 days old, after which they were randomized to a phytoestrogen-free diet or to NIH-07, a diet analyzed as containing a mean concentration of daidzin $81.7 \mu \mathrm{g} / \mathrm{g}$ feed, genistein $95.0 \mu \mathrm{g} / \mathrm{g}$ feed, and glycitein $16.5 \mu \mathrm{g} / \mathrm{g}$ feed (aglycones were not detected). [Assuming a male rat ate $0.07 \mathrm{~kg}$ feed/ $/ \mathrm{kg}$ bw/day (USEPA, 1988), intakes would have been daidzin $5.7 \mathrm{mg} / \mathrm{kg}$ bw/day, genistein $6.6 \mathrm{mg} / \mathrm{kg} \mathrm{bw/day,}$ and glycitein $1.2 \mathrm{mg} / \mathrm{kg}$ bw/day.] Pooled samples from 18 animals/group showed plasma phytoestrogen concentrations on the NIH-07 diet to be daidzein $202.8 \mathrm{ng} / \mathrm{mL}$ (compared to 1.6 $\mathrm{ng} / \mathrm{mL}$ on the phytoestrogen-free diet), genistein $121.0 \mathrm{ng} / \mathrm{mL}$ (compared to $10.5 \mathrm{ng} / \mathrm{mL}$ on the phytoestrogen-free diet), and equol $371.7 \mathrm{ng} / \mathrm{mL}$ (compared to $7.7 \mathrm{ng} / \mathrm{mL}$ on the phytoestrogen-free diet). At 103 days of age, medial-basal hypothalamic/preoptic area and amygdala were dissected from 10 animals/group, and aromatase and $5 \alpha$-reductase activities were measured. Trunk blood was collected for plasma testosterone determination by RIA at the same time brains were collected. Statistical comparisons were made by ANOVA with post- 
hoc Tukey test. No diet-related alterations in feed consumption, body weight, ventral prostate weight, or plasma testosterone concentration were detected. Brain aromatase activity was not shown to be affected by diet in either sampled region. In amygdala, $5 \alpha$-reductase was increased, and in medial-basal hypothalamus/preoptic area, $5 \alpha$-reductase was decreased by the phytoestrogen-containing diet. [The magnitude of the changes was 22-28\%, estimated from a graph.] The authors characterized these alterations as surprising but noted that they were consistent with a prior study that had shown phytoestrogen inhibition of $5 \alpha$-reductase in skin and prostate tissue (Evans et al., 1995).

Strengths/Weaknesses: The experimental design seems adequate, and with the exception of some changes in $5 \alpha$-reductase activity, dietary phytoestrogens had little effect on most other endpoints (e.g., aromatase, plasma testosterone). It is not clear which isoform of $5 \alpha$-reductase was being studied. Type $25 \alpha$-reductase is highly expressed in male accessory sex organs, hair follicles, and genital skin. Only a small amount of testosterone is aromatized, with most enzymatic activity observed in the brain, liver, and adipose tissues. The current findings do little to add to the understanding of how phytoestrogens affect the male reproductive system.

Utility (Adequacy) for CERHR Evaluation Process: These findings need to be confirmed because the overall effect of various phytoestrogens on steroid-metabolizing enzymes needs further investigation. These findings are presently inadequate for interpretation.

Weber et al. (2001b), supported by the National Science Foundation and NIH, evaluated the effects of a soy-phytoestrogen diet on adult male Sprague-Dawley rats. Animals were obtained at 50 days of age and maintained on a standard rat chow containing total phytoestrogens 300 $\mu \mathrm{g} / \mathrm{g}$ feed. At 70 days of age, 114 animals were randomly assigned to one of two commercial diets. One diet was designated phytoestrogen-free and was composed of corn, wheat, fish meal, and wheat middling. The second diet was phytoestrogen-rich and contained soybean meal in place of fish meal. The nutritional content of the two diets was similar. The soy-based diet contained phytoestrogens at about $600 \mu \mathrm{g} / \mathrm{g}$ feed. [Assuming a male rat ate $0.07 \mathbf{~ k g ~ f e e d} / \mathbf{k g}$ bw/day (USEPA, 1988), isoflavone intake in the soy-fed group would have been $42 \mathrm{mg}$ / kg bw/day.] Analysis by HPLC of the soy-based diet showed the phytoestrogens to consist (percentage by weight) of genistein (46\%), daidzin (33\%), glycitein (8\%), daidzein acetylglucoside (5\%), glycitein acetylglucoside (4\%), daidzein (1.7\%), genistein (1.6\%), and glycitein $(0.7 \%)$. [These percentages were derived by CERHR by averaging and rounding duplicate determinations that were reported in the study.] Plasma phytoestrogen concentrations were determined by GC-MS in a subset of 31 rats/diet group after 35 days on their respective diets. Mean total circulating isoflavones were $2224 \mathrm{ng} / \mathrm{mL}$ in the rats fed the soy-based diet and $63 \mathrm{ng} / \mathrm{mL}$ in rats on the phytoestrogen-free diet. On a $\mathrm{ng} / \mathrm{mL}$ basis, of the circulating isoflavones in the soy-based diet group, about $45 \%$ was equol, about $35 \%$ daidzein, and about 20\% genistein. The authors stated that the plasma phytoestrogen concentrations in rats fed the soy-based diet were similar to those in humans on a typical Asian diet and that phytoestrogen concentrations in the rats fed the phytoestrogen-free diet were similar to those in humans on a typical Western diet. After 35 days on the diets, animals were killed. Plasma testosterone, androstenedione, and $17 \beta$-estradiol were determined by RIA in 18 rats/diet group. Ventral prostates were isolated in 13 animals/diet group and $5 \alpha$-reductase activity determined. Testicular tissues from 13 animals/dose group were assayed for steroidogenic acute-regulatory protein by Western analysis. Data, analyzed by ANOVA with post-hoc Tukey test, are summarized in Table 46.

The authors attributed the reduction in prostate weight on the soy-based diet to the decreased concentration of androgens. Because there were no detected effects on LH or steroidogenic acute-regulatory protein (that is involved in cholesterol entry into the steroid pathway), the 
authors could not identify where in the androgen biosynthetic scheme phytoestrogens might be acting.

Strengths/Weaknesses: Most of the endocrine endpoints examined substantiated the estrogenlike effects of the phytoestrogens on the male reproductive system. Reductions in plasma androgens (e.g., testosterone and androstenedione) and prostate weights are consistent with the administration of diets containing genistein and daidzein and are confirmed by the measurements of isoflavones in the two different diets. More studies are needed to understand the action(s) on steroidogenic pathways.

Utility (Adequacy) for CERHR Evaluation Process: Overall, these finding contribute little to understanding the action of isoflavones on the male reproductive system.

Yi et al. (2002), supported by the Korean Science and Engineering Foundation, evaluated the effect of dietary isoflavones on plasma androgens in adult male Sprague-Dawley rats. Rats were fed a basal diet with or without the substitution of soy flour $442.7 \mathrm{~g} / \mathrm{kg}$ feed for corn starch, corn oil, cellulose, and casein. The soy-flour diet contained isoflavones at a concentration of $67.2 \mathrm{mg} / \mathrm{kg}$ feed. The study authors estimated that with a mean feed consumption of $22.4 \mathrm{~g} / \mathrm{day}$, the animals consumed $19 \mathrm{mg} /$ day isoflavones. [This calculation seems to be incorrect. Consumption of $22.4 \mathrm{~g}$ of feed containing isoflavones $0.0672 \mathrm{mg} / \mathrm{g}$ $(67.2 \mathrm{mg} / \mathrm{kg})$ would give a daily isoflavone consumption of $1.5 \mathrm{mg}$, or about $6 \mathrm{mg} / \mathrm{kg}$ bw.] In a second experiment, animals were randomized to AIN-76 diet or to a diet in which corn starch was replaced with soy extract $20 \mathrm{~g} / \mathrm{kg}$ feed (isoflavones $3.38 \mathrm{mg} / \mathrm{g}$ ) or with a semipurified isoflavone preparation $2 \mathrm{~g} / \mathrm{kg}$ feed (isoflavones $218 \mathrm{mg} / \mathrm{g}$ feed). Estimated daily dietary isoflavone intake was $0.9 \mathrm{mg}[\sim 3.6 \mathrm{mg} / \mathbf{k g ~ b w}]$ for the soy-extract diet and $3.3 \mathrm{mg}[\sim$ $13.2 \mathrm{mg} / \mathrm{kg} \mathrm{bw}$ ] for the diet with added semi-purified isoflavones. Animals were placed on their respective diets for 1 week ( $n=6$ or 8/diet group), after which blood was collected for plasma testosterone and dihydrotestosterone by RIA. Plasma isoflavones were determined by HPLC after hydrolysis of the conjugates. Statistical analysis was by ANOVA with Duncan multiple range test.

In the experiment comparing the basal diet to the soy-flour diet, no differences between groups were detected in feed consumption or body weight gain. In the experiment comparing the AIN-76 diet to a diet supplemented with soy extract and a diet supplemented with an isoflavone preparation, feed intake and body weight gain were reduced by the isoflavone preparation (attributed by the authors to taste aversion). The soy-flour diet in the first experiment resulted in a significant reduction in dihydrotestosterone without a detected change in total androgens (testosterone + dihydrotestosterone). In the second experiment, no significant effect of the soy extract-supplemented diet on total androgens or dihydrotestosterone was detected, although the study authors identified a "trend" toward increased androgens. Total androgens were significantly increased and plasma dihydrotestosterone was significantly decreased by the isoflavone preparation. The group that had received the isoflavone preparation also had significantly higher plasma concentrations of genistein and daidzein than the control group, whereas the group that had received the soy extract had plasma isoflavone concentrations that were not identified as significantly different from the control.

The authors concluded that an isoflavone-associated reduction in dihydrotestosterone might be a mechanism by which diets high in soy prevent prostate cancer. They speculated that an increase in plasma testosterone might be compensatory.

Strengths/Weaknesses: The question about the actual calculated dietary consumption of isoflavones is a weakness. Nevertheless, dietary isoflavones led to a decrease in 
dihydrotestosterone, yet had varying effects on total androgens. It is not clear how long the animals received dietary isoflavones.

Utility (Adequacy) for CERHR Evaluation Process: This study is not useful in evaluating a possible relationship between isoflavones and prostate cancer, and it does not contain information that can be used to assess reproductive function in the face of dietary soy.

Faqi et al. (2004), supported by the National Cancer Institute and the IIT Research Institute, evaluated the reproductive effects in male Wistar-Unilever rats of dietary soy isoflavones given for 1 year. The basal feed was Teklad 4\% Fat Rat/Mouse Diet to which was added a soyisoflavone mixture at levels of 0,200 , or $2000 \mathrm{mg} / \mathrm{kg}$ feed [ppm]. The isoflavone mixture contained $45 \%$ genistein, $23 \%$ daidzein, and $4 \%$ glycitein. Analysis of feed samples was carried out monthly and showed the high-dose feed to contain $97.5 \%$ of target isoflavone levels and the control feed to contain isoflavones at $<11 \mathrm{mg} / \mathrm{kg}$ feed (and often undetectable). Based on feed intake and measured isoflavone levels in the diet, high-dose animals were estimated to have ingested isoflavones $80 \mathrm{mg} / \mathrm{kg}$ bw/day during weeks $4-5$ and $75 \mathrm{mg} / \mathrm{kg}$ bw/day during weeks 29-30. During the same periods, isoflavone ingestion in the control group was estimated to have been 0.11 and $0.17 \mathrm{mg} / \mathrm{kg}$ bw/day.

Rats were begun on their assigned diets at 9-10 weeks of age. After 12-13 months, testes and epididymides were removed and weighed. One testis/animal was fixed in Bouin fluid and dehydrated in ethanol. The fixed testis was embedded in paraffin, sectioned at $5 \mu \mathrm{m}$, and stained with hematoxylin and eosin for light microscopy. The other testis was used for counting of homogenization-resistant spermatids. The cauda epididymis was cut into pieces and rinsed with saline to produce a sperm suspension. Sperm morphology was evaluated using eosinstained samples. Statistical analysis used ANOVA with post-hoc Dunnett or Mann-Whitney test. Proportions of morphologically normal sperm were compared using $\chi^{2}$ test.

No treatment effects on mortality, clinical signs, or body weight were detected. Absolute and relative testis weights were not shown to be affected by treatment, and testis histology was comparable in all groups. No treatment group differences in testicular spermatid number and morphologically normal epididymal sperm were detected. The authors concluded that soy isoflavones would be unlikely to adversely affect reproductive function at dose levels comparable to those inhibiting prostate cancer.

Strengths/Weaknesses: This well designed study extended over a 1-year period. The endpoints evaluated were appropriate as part of an evaluation of male reproductive effects.

Utility (Adequacy) for CERHR Evaluation Process: This study is useful in showing a lack of adverse effect of dietary soy isoflavones at up to $2000 \mathrm{mg} / \mathrm{kg}$ feed.

Mäkelä et al. (1995a), supported by the Yrjö Jahnsson Foundation, Turku University Foundation, and Emil Aaltonen Foundation, evaluated the effects of a soy diet on the genital tracts of male Han-NMRI mice that had been estrogenized as neonates with s.c. diethylstilbestrol $2 \mu \mathrm{g} / \mathrm{pup} / \mathrm{day}$ for 3 days. Animals were raised on a soy-free diet. At 3-5 months of age, the animals were castrated and either maintained on the soy-free diet or given a diet containing 7\% roasted soy meal. Some animals in both diet groups were implanted with $17 \beta$-estradiol-containing pellets. After 10 or 21 days, urethroprostatic blocks were sectioned for evaluation by light microscopy. In another experiment, animals on soy diets were treated with $17 \beta$-estradiol, coumestrol, or vehicle, and urethroprostatic tissues were isolated at intervals for detection of $c$-fos mRNA by Northern analysis. Soy-feeding did not produce histologic alterations in the urethroprostatic tissues and did not induce $c$-fos mRNA. There was no apparent effect of dietary soy on the response of the male reproductive tract to $17 \beta$-estradiol. 
The authors concluded that the lack of estrogenic effect of a soy diet in male mice stood in contrast to the apparent estrogenic effect of soy diet in developing female mice.

Strengths/Weaknesses: It is difficult to interpret endocrine effects when the experimental design involved using estrogenized neonates; however, soy-feeding failed to produce histologic changes in urethroprostatic tissues or mRNA in male mice.

Utility (Adequacy) for CERHR Evaluation Process: This study is not useful given the confounding effect of neonatal estrogenization.

\subsection{Utility of Data}

4.3.1 Human data-There are 17 studies on possible human reproductive effects of soy products; 13 involved women and five involved men. None of these studies involved the administration of soy infant formula, and the utility of these studies in the current evaluation is limited by the extent to which other soy products represent soy infant formula. Two of the studies in women (Nagata et al., 1998; Jakes et al., 2001) and one study in men (Nagata et al., 2000) involved ascertainment of typical diets and correlation testing with serum hormone levels. These studies do not directly assess reproductive effects of specific dietary components, but could be useful to infer reproductive effects. Most of the studies involved use of soy foods (tofu, soy milk) added to the diet or the use of soy supplements. These studies also used reproductive hormones and, in some cases, menstrual cycle length as endpoints. Only one study in men (Mitchell et al., 2001), which evaluated semen analysis parameters, had an endpoint directly relevant to the assessment of reproductive toxicity. An important limitation of studies of dietary soy products is the difference between soy food-containing diets and other diets in macronutrient content, which has an independent effect on some hormone levels. Any changes in serum or plasma hormone levels on a soy food- or supplement-containing diet could have been due to these macronutrient changes and not to isoflavones, as suggested by the study of Lu et al. (2001).

4.3.2 Experimental animal data-The study by Boettger-Tong et al. (1998) showed that soy-based diets could exert estrogenic effects in rodents. Additional studies have evaluated estrogenic endpoints in intact and castrated rodents; however, these studies did not necessarily address reproductive function. There are three studies in female rats and there is one study in male rats that address aspects of reproductive function in animals fed diets containing soy isoflavones. A study in cynomolgus monkeys was useful in evaluating possible effects of dietary soy protein on serum $17 \beta$-estradiol, progesterone, menstrual cycle length, uterine area, and vaginal maturation

\subsection{Summary of Reproductive Toxicity Data}

4.4.1 Human data-Studies in women and men are summarized in Table 47 and Table 48.

Diet-intervention trials included the studies of: Cassidy et al. $(1994,1995)$, which used textured vegetable protein, miso, or an isoflavone-free soy product; Lu et al. (1996, 2000a) and Nagata et al. (1998), which used soy milk; Wu et al. (2000) and Maskarinec et al. (2004a), which used a variety of soy foods, and Xu et al. (1998), Martini et al. (1999), Duncan et al. (1999a), and Watanabe et al. (2000), which used a soy supplement. The studies of Nagata et al. (1998), Watanabe et al. (2000), and Maskarinec et al. (2004a) used an untreated control group. The other studies used subjects as their own controls, either with a cross-over design or by sampling blood before, during, and after the intervention. Treatment periods were short, on the order of 1-3 months except for the 2-year study of Maskarinec et al. (2004a). 
The results of the intervention studies in women were generally inconsistent. Length of the menstrual cycle or follicular phase of the menstrual cycle was increased in the studies of Cassidy et al. (1994, 1995), Lu et al. (1996), and Nagata et al. (1998). Menstrual cycle length was unaffected in the studies of Martini et al. (1999), Duncan et al. (1999a), Wu et al. (2000), and Maskarinec et al. (2004a). Luteal-phase length was decreased in a study by Lu et al. (2000a). The isoflavone content of the interventions, although differing from one study to the next, did not explain the discrepancy in cycle lengths. Sample size may have been limiting in the ability to detect significant changes: the two studies showing no effect involved only 14 and 16 regularly cycling subjects. The non-intervention study of Jakes et al. (2001) in which dietary intakes were obtained by questioning and menstrual cycle lengths were obtained from diaries, described longer cycle length as associated with soy-protein intake above the group median.

Serum or plasma estrogens were also affected differently in different studies. An increase in $17 \beta$-estradiol or estrone was associated with soy-product intake in the studies of Cassidy et al. $(1994,1995)$ and Watanabe et al. (2000), and serum estrone correlated with soy-protein intake in the non-intervention study of Jakes et al. (2001). A decrease in serum, plasma, or urinary estrogens was identified in the studies of Lu et al. (1996, 2000a, 2001), Nagata et al. (1998), Xu et al. (1998), Duncan et al. (1999a), and Wu et al. (2000). The non-intervention study of Nagata et al. (1997) found a negative association between soy products in the diet and serum $17 \beta$-estradiol in the second half of the cycle. One of the studies of Lu et al. (2001) found the decrease in serum $17 \beta$-estradiol on a reduced-isoflavone soy supplement to lose statistical significance when adjustments were made for the macronutrient intake of the subjects. The 2year study of Maskarinec et al. (2004a) did not detect an effect of dietary soy products on sex hormones or sex hormone-binding globulin in women.

Studies in men used dietary additions of tofu (Habito et al., 2000), soy milk (Nagata et al., 2001), or a soy supplement (Mitchell et al., 2001). Interventions were applied for 1 or 2 months. These studies did not detect an effect of the intervention on serum testosterone or 17 $\beta$-estradiol, although one of them (Nagata et al., 2001) reported a decrease in serum estrone with soy milk added to the diet. The non-intervention study of Nagata et al. (2000) reported an inverse correlation between serum 17 $\beta$-estradiol in men and intake of soy products. Habito et al. (2000) reported a significant decrease in the testosterone:estradiol ratio and an increase in sex hormone-binding globulin after addition of tofu to the diet for 1 month. Both of these hormone changes might be expected to decrease androgen activity; however, no measures of androgen activity were included in the study. Mitchell et al. (2001) did not detect an effect of a daily soy supplement with $40 \mathrm{mg}$ isoflavones on testicular volume or semen analysis parameters after 2 months of treatment. There were only 15 subjects in this study.

4.4.2 Experimental animal data-Gallo et al. (1999) added isoflavones to a solventextracted commercial-soy diet at levels of $0.7,1.2$, and $2.4 \%$ [estimated to produce daily isoflavone consumption of about 82, 140, and $290 \mathrm{mg} / \mathrm{kg}$ bw]. Female Wistar rats began treatment at weaning and were mated on PND 77. Dams were permitted to litter, and pups were observed until PND 7. Estrogenic changes in the reproductive tract of the dams were apparent at all isoflavone levels. An increase in uterine weight and a decrease in small ovarian follicles were the most sensitive endpoints. Despite the clear evidence of estrogenic effect in the genital tract, there was no apparent alteration in fertility or the ability to bear young and raise them until PND 7.

Two studies by Patisaul et al. (2001) found dietary isoflavones to antagonize the action of estradiol benzoate and $17 \beta$-estradiol in the sexual response of ovariectomized Long-Evans rats. The treatment used a soy supplement at a dietary concentration of $0.35 \%$, providing an estimated total genistein concentration of $13 \mathrm{ppm}(1.04 \mathrm{mg} / \mathrm{kg}$ bw/day $)$ and an estimated total 
daidzein concentration of $33 \mathrm{ppm}(2.64 \mathrm{mg} / \mathrm{kg}$ bw/day). Plasma phytoestrogens, which were not measured in these studies, were estimated to be in the range of human dietary exposures. The anti-estrogenic effect of the isoflavone-containing diet on sexual function was similar to that of tamoxifen.

A study by Faqi et al. (2004) used a defined mixture of soy isoflavones administered to male Wistar-Unilever rats in the diet at 200 or $2000 \mathrm{mg} / \mathrm{kg}$ feed [ppm] for 12-13 months. No adverse effects of treatment were observed on body weight, weight of the testis or epididymis, sperm morphology, homogenization-resistant spermatid number, daily sperm production, or testis histopathology. The high-dose group was estimated to have consumed isoflavones $75-80 \mathrm{mg} /$ $\mathrm{kg}$ bw/day.

Wood et al. (2006), fed adult female cynomolgus monkeys a soy protein diet $(\mathrm{n}=27)$ containing isoflavones $1.88 \mathrm{mg} / \mathrm{g}$ protein, which the authors estimated as equivalent to human consumption of total genistein $91 \mathrm{mg}$, total daidzein $31 \mathrm{mg}$, and total glycitein $7 \mathrm{mg}$ for an $1800 \mathrm{kcal}$ diet. A control group was given a casein-lactalbumin diet. After 12 months, no effect of diet was observed on serum 17 $\beta$-estradiol or progesterone, menstrual cycle length, uterine area, or vaginal maturation.

\section{Conclusions of the Expert Panel}

Evidence is insufficient to conclude that soy infant formula would or would not produce reproductive toxicity in men or women.

Evidence is insufficient to conclude that soy infant formula would or would not produce reproductive toxicity in experimental animals.

Note: The definitions of the term sufficient and the terms assumed relevant, relevant, and not relevant are in the CERHR guidelines at

http://cerhr.niehs.nih.gov/news/guidelines.html.

\subsection{SUMMARIES, CONCLUSIONS, AND CRITICAL DATA NEEDS}

\subsection{Summary and Conclusions of Reproductive and Developmental Hazards}

5.1.1 Soy formula developmental toxicity data-There are no data in humans on the effects of prenatal exposure to soy infant formula, but there are several studies that examined the developmental effects of childhood exposures to soy infant formula. These studies suggest no effects on growth (height, weight, and body mass index) or sexual maturation (e.g., precocious puberty or gynecomastia); however, the sample sizes in these studies may have been insufficient to detect small changes or effects occurring at low incidence. Infants with a familial predisposition to develop allergies (and without soy hypersensitivity) apparently did not differ in their incidence of allergies when given soy or cow-milk formula. In these cases, breast milk was superior to either formula. In hypothyroid infants, a few case reports suggest that soy infant formula may affect absorption of administered thyroxine. Soy infant formula may not adequately support growth in premature infants, a subpopulation for which the use of soy infant formula is not recommended. One study (Freni-Titulaer et al., 1986) showed that soy formula feeding was a risk factor for premature breast development in girls under 2 years of age. Overall, the data are limited by small sample sizes, conflicting results, and historical studies that used soy infant formula of different composition than the formula being sold currently.

There are no experimental animal data on the effects of prenatal exposures to soy infant formula. The developmental toxicity of soy formula has been assessed in a single study with marmosets that were partially formulafed, receiving soy infant formula 2-8 hr/day from 4-5 
days of age until 5-6 weeks of age (Sharpe et al., 2002; Tan et al., 2006). In comparison with co-twins fed cow-milk formula, the marmosets receiving soy milk had a transient decrease in plasma testosterone on PND 35-45 and a subsequent increase in testis weight, and Sertoli and Leydig cell numbers at 120-138 weeks of age. Several rodent studies have examined the effects of isoflavone-, phytoestrogen-, or soybean-containing diets after exposure from prenatal stages through sexual maturity. Although some studies suggested effects on the reproductive system development, these results often conflicted with the results of other studies. Interpretation of these data was limited by the use of a single dose level and insufficient control for differences in rodent diets. The Expert Panel notes that the rodents in these studies were not dosed with soy infant formula. Furthermore, animal models used in these experiments metabolize soy differently than human infants, forming the estrogenic daidzein metabolite equol.

The Expert Panel acknowledges the difficulty in establishing an animal model to simulate feeding doses and duration similar to that observed in humans. Therefore, the adverse findings described in animal models, which could be attributed to differences in metabolism, diet, limitation of dose, lack of use of soy infant formula among others, may not lend themselves to interpretation of adverse effects in humans.

\subsubsection{Soy formula reproductive toxicity data}

5.1.2.1 Male effects: Soy product consumption has not been consistently associated with reproductive effects in men. In diet intervention trials, no significant association was observed between soy product exposure and circulating testosterone or 17 $\beta$-estradiol levels (Habito et al., 2000; Mitchell et al., 2001). In contrast, soy milk consumption was associated with a decrease in serum estrone levels (Nagata et al., 2001) and intake of soy products was negatively correlated with serum 17ß-estradiol levels (Nagata et al., 2000). One study observed no significant association between soy consumption and testicular volume or semen parameters.

Only one study has examined the effects of soy product exposure on male reproduction in experimental animals. This study did not detect an effect of soy product exposure on body weight, testicular or epididymal weight, sperm morphology and production, spermatid number, or testis histopathology in rats (Faqi et al., 2004).

5.1.2.2 Female effects: Soy product consumption has not been associated consistently with reproductive effects in women. Some studies observed an association between soy product consumption and increased menstrual cycle length (Cassidy et al., 1994, 1995; Lu et al., 1996; Nagata et al., 1998; Jakes et al., 2001), whereas other studies did not observe an association (Duncan et al., 1999a; Martini et al., 1999; Wu et al., 2000; Maskarinec et al., 2004a), and one study observed an association with decreased luteal phase length (Lu et al., 2000a). In addition, soy product consumption has not been consistently associated with changes in circulating endogenous estrogens. Some studies observed an association between soy product consumption and increased 17 $\beta$-estradiol and estrone levels (Cassidy et al., 1994, 1995; Watanabe et al., 2000; Jakes et al., 2001), whereas others observed an association with decreased estrogen levels (Lu et al., 1996, 2000a, 2001; Nagata et al., 1998; Xu et al., 1998; Duncan et al., 1999a; Wu et al., 2000), and one study did not observe an association between soy consumption and sex hormones or sex hormone-binding globulin (Maskarinec et al., 2004a).

Only a few studies have examined the effects of soy product exposure on female reproduction in experimental animals (Gallo et al., 1999; Patisaul et al., 2001; Wood et al., 2006). One study in rats observed an effect of soy product exposure on uterine weight and small follicle numbers, but not on fertility and ability to bear and raise young (Gallo et al., 1999). Given the effect of soy product exposure on small follicle numbers, there is a possibility that the animals could have a shortened reproductive lifespan, although this possibility was not directly examined in 
the study. Other studies suggest that soy product exposure may antagonize the effects of 17ßestradiol on sexual behavior in rats (Patisaul et al., 2001, 2004). In addition, one study observed no effect of soy exposure on serum $17 \beta$-estradiol or progesterone levels, menstrual cycle length, or uterine or vaginal morphology in monkeys (Wood et al., 2006).

Although no experimental animals in reproductive studies have been exposed to soy formula, the Expert Panel noted that no reproductive effects were observed in female and male rats or female monkeys at total dietary isoflavone concentrations estimated to be similar or substantially higher (approximately 10 times) than those normally consumed in soy formula by infants.

\subsection{Summary of Human Exposure}

Soy formula refers to infant food made using soy protein isolate and other components such as corn syrup, vegetable oils, and sugar (Drugstore.com, 2004). The soy protein isolate is fortified with L-methionine, L-carnitine, and taurine (AAP, 1998). Contaminants of soy protein include phytates $(1.5 \%)$, which bind minerals, and protease inhibitors, which have antitrypsin, antichymotrypsin, and anti elastin properties (AAP, 1998). Heat applied during the processing of soy protein removes $80-90 \%$ of protease inhibitor activity. Phosphorus, calcium, iron, and zinc are added to soy formula to compensate for phytate binding of minerals. Aluminum is present in soy formulas because of the addition of mineral salts.

Soy products contain phytoestrogens of the isoflavone class (MAFF, 1998b; Setchell et al., 1998; UK Committee on Toxicity, 2003). Isoflavone levels in soybeans are reported to vary as a result of geographic location, climate, and growing conditions (Setchell et al., 1998). The primary isoflavones detected in soybeans are derived from genistein, daidzein, and to a smaller extent, glycitein. Genistein, daidzein, glycitein and their conjugates have been detected in soy infant formula (MAFF, 1998b; Setchell et al., 1998). The majority (>65\%) of isoflavones in soy formula are conjugated to sugar molecules to form glycosides (Setchell et al., 1998). Glucose groups in glycosides can be esterified with acetyl or malonyl groups to form acetylor malonyl glycosides (UK Committee on Toxicity, 2003). Small amounts of genistein and daidzein (3.2-5.8\%) are present in soy products in their unconjugated (aglycone) forms (Chen and Rogan, 2004). Because glycosidic compounds can be deconjugated in the gut wall to form the biologically active aglycone, exposure to a particular isoflavone (e.g., genistein) is theoretically the sum of the aglycone and respective glycoside concentrations converted on the basis of molecular weight (MAFF, 1998b; Setchell et al., 1998; UK Committee on Toxicity, 2003). However, the aglycone is reconjugated in the gut wall leaving approximately $1-2 \%$ free aglycone to enter the portal circulation.

An estimated 10-20\% of infants in the U.S. are fed soy infant formula some time during the first year of life (Essex, 1996; Strom et al., 2001). Soy infant formulas are generally used to feed infants who are allergic to dairy products or are intolerant of lactose, galactose, or cowmilk protein (Essex, 1996; Tuohy, 2003). Infants are sometimes given soy infant formula when they have symptoms such as colic, crying, diarrhea, or vomiting (Forsyth et al., 1985) or to maintain a vegetarian or perceived healthy lifestyle (Badger et al., 2002). Soy formula is not recommended currently for preterm infants.

A number of studies measured total isoflavone (aglycone + glycoside) levels in infant formulas, determined percentages of individual isoflavones, or estimated infant exposures. In a USDA survey, levels reported in reconstituted formulas or ready-to-feed formulas were 26.3-41.7 $\mu \mathrm{g}$ total isoflavone equivalents/g formula, 15.8-22.6 $\mu \mathrm{g}$ genistein equivalents/g formula, 7.5-19.1 $\mu \mathrm{g}$ daidzein equivalents/g formula, and 2.8-3.5 $\mu \mathrm{g}$ glycitein equivalents/g formula (USDA, 2002b). On a mg isoflavone equivalents/L ready-to-feed formula basis, total isoflavone levels were $25-47 \mathrm{mg} / \mathrm{L}$ in U.S. soy formulas (Murphy et al., 1997; Setchell et al., 1998) except for 
one study reporting a total isoflavone level of $155-281 \mathrm{mg} / \mathrm{L}$ (Franke et al., 1998), and 18-41 $\mathrm{mg} / \mathrm{L}$ in formulas obtained from the U.K. and Australia (Knight et al., 1998; MAFF, 1998a,b). Percentages of individual isoflavones in U.S. soy formulas ranged from 36.8-70.1\% genistein equivalents, $18.2-45.8 \%$ daidzein equivalents, and 5-13.0\% glycitein equivalents (Murphy et al., 1997; Setchell et al., 1997, 1998; Franke et al., 1998; USDA, 2002b). Percentages of individual isoflavones in formulas from the U.K. were reported at 58-67\% genistein equivalents, $27-36 \%$ daidzein equivalents, and 6-16\% glycitein equivalents (MAFF, 1998a,b; Hoey et al., 2004).

Isoflavone exposure through soy infant formula intake in infants has been estimated based on total isoflavone levels measured in soy infant formulas from several countries, and assumptions of formula intakes and infant body weights (Table 49; data taken from Table 6 in Section 1.0).

Mean \pm SD plasma levels of total isoflavones in infants fed soy formula were reported at 683 $\pm 442.6 \mu \mathrm{g} / \mathrm{L}$ for genistein equivalents, $295.3 \pm 59.9 \mu / \mathrm{L}$ for daidzein equivalents, and $552-1775$ $\mu \mathrm{g} / \mathrm{L}$ (mean $980 \mu \mathrm{g} / \mathrm{L}$ ) for total isoflavones (Setchell et al., 1997, 1998). Mean plasma total isoflavone levels were $\sim 2$ orders of magnitude higher than in infants fed cow-milk formula or human milk. Mean plasma levels in infants fed soy infant formulas were $\sim 5-20$ times higher than in Japanese adults or adults ingesting similar levels of total isoflavones from soy-based foods, $\sim 50$ times higher than in vegetarian adults in Western populations, and $\sim 500$ times higher than in omnivorous adults in Western populations (Setchell et al., 1997, 1998). Dietary exposure estimates comparing total isoflavone intake in infants ingesting soy formula compared to adults are consistent with comparisons based on plasma total isoflavone levels (Rozman et al., in press).

\subsection{Overall Conclusions}

There are insufficient human or experimental animal data available to permit determination of developmental or reproductive toxicity of soy infant formula. Some of the available human studies suffer from poor design, inadequate description, or insufficient sample size to permit confidence in the observed results. One large longitudinal study following a cohort of 811 individuals who were fed either cow-milk or soy infant formula as infants showed no significant effects on a variety of reproductive and developmental endpoints, e.g., body weight, in females, age at menarche, age at breast development, cycle length, duration of menses, and in males, age at first ejaculation, age when hair developed on chest, or age when voice changed (Strom et al., 2001). A number of the studies present findings that deserve further careful examination, including a study showing that soy infant formula feeding was a risk factor for premature breast development in girls under age 2 years (Freni-Titulaer et al., 1986).

None of the rodent studies evaluated were carried out using exposure to soy infant formula, and nearly all were done using soy products added to animal chow. Additionally, most of these studies suffered from poor experimental design with common deficiencies being small numbers of animals per treatment group and examination of single dose levels of soy products. Single studies evaluated reproductive endpoints in either male or female rats or female cynomolgus monkeys; in these studies total dietary isoflavone concentrations were estimated to be similar to or substantially (approximately 10 times) higher than those normally consumed by infants fed soy infant formula. These studies did not identify adverse effects for most of the reproductive endpoints that were examined; however, there was a significant decrease in small ovarian follicle number in one study in rats (Gallo et al., 1999). 


\subsection{Critical Data Needs}

Critical data needs are defined as tests or measurements that could provide information to substantially improve an assessment of human reproductive and developmental risks. The items listed below are considered by the Expert Panel as critical data needs:

Data are needed to describe more carefully human infant exposure to isoflavones in soy infant formula using biomarkers of exposure. These studies should consider use of formula type, concomitant ingestion of other soy-containing compounds, and length of exposure.

Another case-control study to examine premature breast development in females and exposure to soy infant formula is needed. This study should be large enough to ensure sufficient statistical power to detect meaningful differences.

A longitudinal cohort study to examine postnatal growth and neurobehavioral development of healthy, full-term infants fed soy infant formula; these infants should be compared to breastfed or cow milk formula-fed infants, with particular attention paid to exposure conditions. This study should be large enough to ensure sufficient statistical power to detect meaningful differences.

Case-control studies are needed to examine reproductive endpoints, such as age at beginning of puberty, early age at onset of menopause, endometriosis, uterine leiomyomata, and reproductive organ carcinogenesis and neonatal exposure to soy infant formula and other soy products. These studies should be large enough to ensure sufficient statistical power to detect meaningful differences. Longitudinal cohort studies should be identified that have the potential to evaluate exposure to soy infant formula in relation to these outcomes, including age at onset of menopause.

A carefully controlled animal study is needed in which multiple doses of soy infant formula or other soy products are used so that dose-response relationships can be determined. Careful consideration should be given to the choice of animal model, mindful of metabolic differences between species particularly in the formation of equol. Consideration should also be given to the appropriateness of this animal model to the human neonate. This study could be of two parts, with one part consisting of prenatal exposure only and evaluation of developmental endpoints; the second part could be a multigenerational study with exposure continuing into adulthood and evaluation of both reproductive and postnatal developmental endpoints. Nutritional differences in animal diets need to be considered in these experiments.

A carefully controlled animal study is needed in which the effects of soy infant formula or other soy products on ovarian follicle counts and early ovarian failure are evaluated.

\section{Acknowledgements}

Prepared With the Support of CERHR Staff: NTP/NIEHS, Michael Shelby, Ph.D. (Director, CERHR), Paul M.D. Foster, Ph.D. (Deputy Director, CERHR), Allen Dearry, Ph.D. (Interim Associate Director, NTP), Kristina Thayer, Ph.D. (NTP Liaison and Scientific Review Office); Sciences International, Inc., Anthony Scialli, M.D. (Principal Scientist), Annette Iannucci, M.S. (Toxicologist), Gloria Jahnke, D.V.M. (Toxicologist), Vera Jurgenson, M.S. (Research Associate).

This report is prepared according to the Guidelines for CERHR Panel Members established by NTP/NIEHS. The guidelines are available on the CERHR web site (http://cerhr.niehs.nih.gov/). The format for Expert Panel Reports includes synopses of studies reviewed, followed by an evaluation of the Strengths/Weaknesses and Utility (Adequacy) of the study for CERHR evaluation. Statements and conclusions made under Strengths/Weaknesses and Utility evaluations are those of the Expert Panel and are prepared according to the NTP/NIEHS guidelines. In addition, the Panel often makes comments or notes limitations in the synopses of the study. Bold, square brackets are used to enclose such statements. As discussed in the guidelines, square brackets are used to enclose key items of information not provided in a publication, limitations noted in the study, conclusions that differ from those of the authors, and conversions or analyses of data conducted by the Panel. 
The findings and conclusions of this report are those of the expert panel and should not be construed to represent the views of the National Toxicology Program.

\section{REFERENCES}

AAP. Soy-protein formulas: recommendations for use in infant feeding. Pediatrics 1998;101:148-153. [PubMed: 11345979]

Adlercreutz H. Phyto-oestrogens and cancer. Lancet Oncol 2002;3:364-373. [PubMed: 12107024]

Adlercreutz H, Fotsis T, Lampe J, Wahala K, Makela T, Brunow G, Hase T. Quantitative determination of lignans and isoflavonoids in plasma of omnivorous and vegetarian women by isotope dilution gas chromatography-mass spectrometry. Scand J Clin Lab Invest Suppl 1993a;215:5-18. [PubMed: 8392221]

Adlercreutz H, Markkanen H, Watanabe S. Plasma concentrations of phyto-oestrogens in Japanese men. Lancet 1993b;342:1209-1210. [PubMed: 7901532]

Adlercreutz H, Yamada T, Wahala K, Watanabe S, Businco L, Bruno G, Giampietro PG, Cantani A, Ferrara M, Ragno V. Maternal and neonatal phytoestrogens in Japanese women during birth. Am J Obstet Gynecol 1999;180:737-743. [PubMed: 10076156]

Ament ME, Rubin CE. Soy protein-another cause of the flat intestinal lesion. Gastroenterology 1972;62:227-234. [PubMed: 4674035]

Anthony MS, Clarkson TB, Hughes CL Jr, Morgan TM, Burke GL. Soybean isoflavones improve cardiovascular risk factors without affecting the reproductive system of peripubertal rhesus monkeys. J Nutr 1996;126:43-50. [PubMed: 8558324]

Arai Y, Uehara M, Sato Y, Kimira M, Eboshida A, Adlercreutz H, Watanabe S. Comparison of isoflavones among dietary intake, plasma concentration and urinary excretion for accurate estimation of phytoestrogen intake. J Epidemiol 2000;10:127-135. [PubMed: 10778038]

Ashby J, Tinwell H, Odum J. Uterotrophic activity of a "phytoestrogen-free" rat diet. Environ Health Perspect 2000a;108:A12-A13. [PubMed: 10620531]

Ashby J, Tinwell H, Odum J, Kimber I, Brooks AN, Pate I, Boyle CC, Setchell KD, Zimmer-Nechemias L, Cai J, Heubi JE. Diet and the aetiology of temporal advances in human and rodent sexual development Isoflavone content of infant formulas and the metabolic fate of these phytoestrogens in early life. J Appl Toxicol 2000b;20:343-347. [PubMed: 11139164]

Atanassova N, McKinnell C, Turner KJ, Walker M, Fisher JS, Morley M, Millar MR, Groome NP, Sharpe RM. Comparative effects of neonatal exposure of male rats to potent and weak (environmental) estrogens on spermatogenesis at puberty and the relationship to adult testis size and fertility: evidence for stimulatory effects of low estrogen levels. Endocrinology 2000;141:3898-3907. [PubMed: 11014247]

Badger TM, Ronis MJ, Hakkak R, Rowlands JC, Korourian S. The health consequences of early soy consumption. J Nutr 2002;132:559S-565S. [PubMed: 11880593]

Baird DD, Umbach DM, Lansdell L, Hughes CL, Setchell KD, Weinberg CR, Haney AF, Wilcox AJ, McLachlan JA. Dietary intervention study to assess estrogenicity of dietary soy among postmenopausal women. J Clin Endocrinol Metab 1995;80:1685-1690. [PubMed: 7745019]

Berger-Achituv S, Shohat T, Romano-Zelekha O, Ophir E, Rachmani S, Malovizky D, Garty BZ. Widespread use of soy-based formula without clinical indications. J Pediatr Gastroenterol Nutr 2005;41:660-666. [PubMed: 16254527]

Bloedon LT, Jeffcoat AR, Lopaczynski W, Schell MJ, Black TM, Dix KJ, Thomas BF, Albright C, Busby MG, Crowell JA, Zeisel SH. Safety and pharmacokinetics of purified soy isoflavones: single-dose administration to postmenopausal women. Am J Clin Nutr 2002;76:1126-1137. [PubMed: 12399289]

Boettger-Tong H, Murthy L, Chiappetta C, Kirkland JL, Goodwin B, Adlercreutz H, Stancel GM, Makela $\mathrm{S}$. A case of a laboratory animal feed with high estrogenic activity and its impact on in vivo responses to exogenously administered estrogens. Environ Health Perspect 1998;106:369-373. [PubMed: 9637793]

Bouker KB, Hilakivi-Clarke L. Genistein: does it prevent or promote breast cancer? Environ Health Perspect 2000;108:701-708. [PubMed: 10964789] 
Brown NM, Setchell KD. Animal models impacted by phytoestrogens in commercial chow: implications for pathways influenced by hormones. Lab Invest 2001;81:735-747. [PubMed: 11351045]

Burks AW, Casteel HB, Fiedorek SC, Williams LW, Pumphrey CL. Prospective oral food challenge study of two soybean protein isolates in patients with possible milk or soy protein enterocolitis. Pediatr Allergy Immunol 1994;5:40-45. [PubMed: 8173638]

Busby MG, Jeffcoat AR, Bloedon LT, Koch MA, Black T, Dix KJ, Heizer WD, Thomas BF, Hill JM, Crowell JA, Zeisel SH. Clinical characteristics and pharmacokinetics of purified soy isoflavones: single-dose administration to healthy men. Am J Clin Nutr 2002;75:126-136. [PubMed: 11756070]

Callenbach JC, Sheehan MB, Abramson SJ, Hall RT. Etiologic factors in rickets of very low-birth-weight infants. J Pediatr 1981;98:800-805. [PubMed: 7229765]

Cassidy A, Bingham S, Setchell K. Biological effects of isoflavones in young women: importance of the chemical composition of soyabean products. Br J Nutr 1995;74:587-601. [PubMed: 7577895]

Cassidy A, Bingham S, Setchell KD. Biological effects of a diet of soy protein rich in isoflavones on the menstrual cycle of premenopausal women. Am J Clin Nutr 1994;60:333-340. [PubMed: 8074062]

Cassidy A, Brown JE, Hawdon A, Faughnan MS, King LJ, Millward J, Zimmer-Nechemias L, Wolfe B, Setchell KD. Factors affecting the bioavailability of soy isoflavones in humans after ingestion of physiologically relevant levels from different soy foods. J Nutr 2006;136:45-51. [PubMed: 16365057]

Chan GM, Leeper L, Book LS. Effects of soy formulas on mineral metabolism in term infants. Am J Dis Child 1987;141:527-530. [PubMed: 3578165]

Chandra RK. Five-year follow-up of high-risk infants with family history of allergy who were exclusively breast-fed or fed partial whey hydrolysate, soy, and conventional cow's milk formulas. J Pediatr Gastroenterol Nutr 1997;24:380-388. [PubMed: 9144119]

Chandra RK. Five-year follow up of high-risk infants with family history of allergy exclusively breastfed or fed partial whey hydrolysate, soy and conventional cow's milk formulas. Nutr Res 1998;18:1395-1411.

Chandra RK, Hamed A. Cumulative incidence of atopic disorders in high risk infants fed whey hydrolysate, soy, and conventional cow milk formulas. Ann Allergy 1991;67:129-132. [PubMed: 1867449]

Chandra RK, Puri S, Hamed A. Influence of maternal diet during lactation and use of formula feeds on development of atopic eczema in high risk infants. BMJ 1989a;299:228-230. [PubMed: 2504375]

Chandra RK, Singh G, Shridhara B. Effect of feeding whey hydrolysate, soy and conventional cow milk formulas on incidence of atopic disease in high risk infants. Ann Allergy 1989b;63:102-106. [PubMed: 2669565]

Chang HC, Doerge DR. Dietary genistein inactivates rat thyroid peroxidase in vivo without an apparent hypothyroid effect. Toxicol Appl Pharmacol 2000;168:244-252. [PubMed: 11042097]

Chapin RE, Gulati DK, Barnes LH, Teague JL. The effects of feed restriction on reproductive function in Sprague-Dawley rats. Fundam Appl Toxicol 1993;20:23-29. [PubMed: 8432425]

Chen A, Rogan WJ. Isoflavones in soy infant formula: a review of evidence for endocrine and other activity in infants. Annu Rev Nutr 2004;24:33-54. [PubMed: 15189112]

Cherry FF, Cooper MD, Stewart RA, Platou RV. Cow versus soy formulas. Comparative evaluation in normal infants. Am J Dis Child 1968;115:677-692. [PubMed: 5694767]

Chorazy PA, Himelhoch S, Hopwood NJ, Greger NG, Postellon DC. Persistent hypothyroidism in an infant receiving a soy formula case report and review of the literature. Pediatrics 1995;96:148-150. [PubMed: 7596704]

Churella HR, Borschel MW, Thomas MR, Breen M, Jacobs J. Growth and protein status of term infants fed soy protein formulas differing in protein content. J Am Coll Nutr 1994;13:262-267. [PubMed: 8077575]

Churella HR, Yao BC, Thomson WA. Soybean trypsin inhibitor activity of soy infant formulas and its nutritional significance for the rat. J Agric Food Chem 1976;24:393-397. [PubMed: 943432]

Clark, RL. Endpoints of reproductive system development. An evaluation and interpretation of reproductive endpoints for human health assessment. Washington, DC: International Life Sciences Institute; 1999. p. 10-27. 
Cline JM, Paschold JC, Anthony MS, Obasanjo IO, Adams MR. Effects of hormonal therapies and dietary soy phytoestrogens on vaginal cytology in surgically postmenopausal macaques. Fertil Steril 1996;65:1031-1035. [PubMed: 8612830]

Cordle CT, Winship TR, Schaller JP, Thomas DJ, Buck RH, Ostrom KM, Jacobs JR, Blatter MM, Cho $\mathrm{S}$, Gooch WM 3rd, Pickering LK. Immune status of infants fed soy-based formulas with or without added nucleotides for 1 year: part 2: immune cell populations. J Pediatr Gastroenterol Nutr 2002;34:145-153. [PubMed: 11840031]

Coughtrie MW, Burchell B, Leakey JE, Hume R. The inadequacy of perinatal glucuronidation: immunoblot analysis of the developmental expression of individual UDP-glucuronosyltransferase isoenzymes in rat and human liver microsomes. Mol Pharmacol 1988;34:729-735. [PubMed: 3143908]

Cruz MLA, Wong WW, Mimouni F, Hachey DL, Setchell KD, Klein PD, Tsang RC. Effects of infant nutrition on cholesterol synthesis rates. Pediatr Res 1994;35:135-140. [PubMed: 8165045]

Dean ME. Study of normal infants fed a soya protein isolate formula. Med J Aust 1973;1:1289-1293. [PubMed: 4740892]

Doerge DR, Chang HC, Churchwell MI, Holder CL. Analysis of soy isoflavone conjugation in vitro and in human blood using liquid chromatography-mass spectrometry. Drug Metab Dispos 2000;28:298307. [PubMed: 10681374]

Doerge DR, Twaddle NC, Banks EP, Jefferson WN, Newbold RR. Pharmacokinetic analysis in serum of genistein administered subcutaneously to neonatal mice. Cancer Lett 2002;184:21-27. [PubMed: 12104044]

Döhler K-D, Wong CC, von xur Mühlen A. The rat as model for the study of drug effects on thyroid function: consideration of methodological problems. Clin Pharmacol Ther 1979;5:305-318.

Drugstore.com. Formulation information for Isomil, Isomil Advance, Isomil 2, Enfamil ProSobee, and Enfamil NextStep soy formulations. 2004. Available at drugstore.com.

Duncan AM, Merz BE, Xu X, Nagel TC, Phipps WR, Kurzer MS. Soy isoflavones exert modest hormonal effects in premenopausal women. J Clin Endocrinol Metab 1999a;84:192-197. [PubMed: 9920082]

Duncan AM, Underhill KE, Xu X, Lavalleur J, Phipps WR, Kurzer MS. Modest hormonal effects of soy isoflavones in postmenopausal women. J Clin Endocrinol Metab 1999b;84:3479-3484. [PubMed: 10522983]

Engel SM, Levy B, Liu Z, Kaplan D, Wolff MS. Xenobiotic phenols in early pregnancy amniotic fluid. Reprod Toxicol 2006;21:110-112. [PubMed: 16112541]

Essex C. Phytoestrogens and soy based infant formula: risks remain theoretical. Br Med J 1996;313:507508. [PubMed: 8789965]

Evans BA, Griffiths K, Morton MS. Inhibition of 5 alpha-reductase in genital skin fibroblasts and prostate tissue by dietary lignans and isoflavonoids. J Endocrinol 1995;147:295-302. [PubMed: 7490559]

Faqi AS, Johnson WD, Morrissey RL, McCormick DL. Reproductive toxicity assessment of chronic dietary exposure to soy isoflavones in male rats. Reprod Toxicol 2004;18:605-611. [PubMed: 15135855]

FDA. FDA, 21 CFR Part 101. Food labeling: health claims; soy protein and coronary heart disease. Federal Register 1999; 64 FR 57699. 1999. Available at http://www.cfsan.fda.gov/ lrd/fr991026.html.

FDA. Infant formula. 21 CFR 107. 2000.

Fitzpatrick M. Soy formulas and the effects of isoflavones on the thyroid. N Z Med J 2000;113:24-26. [PubMed: 11482324]

Fomon SJ. Comparative study of human milk and a soya bean formula in promoting growth and nitrogen retention by infants. Pediatrics 1959;24:577-584. [PubMed: 13823791]

Forsyth BW, McCarthy PL, Leventhal JM. Problems of early infancy formula changes and mothers beliefs about their infants. J Pediatr 1985;106:1012-1017. [PubMed: 3998939]

Fort P, Lanes R, Dahlem S, Recker B, Weyman-Daum M, Pugliese M, Lifshitz F. Breast feeding and insulin-dependent diabetes mellitus in children. J Am Coll Nutr 1986;5:439-441. [PubMed: 3155358] 
Fort P, Moses N, Fasano M, Goldberg T, Lifshitz F. Breast and soy-formula feedings in early infancy and the prevalence of autoimmune thyroid disease in children. J Am Coll Nutr 1990;9:164-167. [PubMed: 2338464]

Foster WG, Chan S, Platt L, Hughes CL Jr. Detection of phytoestrogens in samples of second trimester human amniotic fluid. Toxicol Lett 2002a;129:199-205. [PubMed: 11888703]

Foster WG, Hughes CL, Chan S, Platt L. Human developmental exposure to endocrine active compounds. Environ Toxicol Pharmacol 2002b;12:75-81.

Foth D, Nawroth F. Effect of phytoestrogens on the endometrium? Fertil Steril 2005;83:256-257. [PubMed: 15652928]

Franke AA, Custer LJ. Daidzein and genistein concentrations in human milk after soy consumption. Clin Chem 1996;42:955-964. [PubMed: 8665689]

Franke AA, Custer LJ, Tanaka Y. Isoflavones in human breast milk and other biological fluids. Am J Clin Nutr 1998;68:1466S-1473S. [PubMed: 9848518]

Freni-Titulaer LW, Cordero JF, Haddock L, Lebron G, Martinez R, Mills JL. Premature thelarche in Puerto Rico. A search for environmental factors. Am J Dis Child 1986;140:1263-1267. [PubMed: 3776944]

Gallo D, Cantelmo F, Distefano M, Ferlini C, Zannoni GF, Riva A, Morazzoni P, Bombardelli E, Mancuso S, Scambia G. Reproductive effects of dietary soy in female Wistar rats. Food Chem Toxicol 1999;37:493-502. [PubMed: 10456677]

Giampietro PG, Bruno G, Furcolo G, Casati A, Brunetti E, Spadoni GL, Galli E. Soy protein formulas in children: no hormonal effects in long-term feeding. J Pediatr Endocrinol Metab 2004;17:191-196. [PubMed: 15055353]

Goldman LR, Newbold R, Swan SH. Exposure to soy-based formula in infancy. JAMA 2001;286:24022403. [PubMed: 11712933]

Golub MS, Hogrefe CE, Germann SL, Tran TT, Beard JL, Crinella FM, Lonnerdal B. Neurobehavioral evaluation of rhesus monkey infants fed cow's milk formula, soy formula, or soy formula with added manganese. Neurotoxicol Teratol 2005;27:615-627. [PubMed: 15955660]

Gruskay FL. Comparison of breast, cow, and soy feedings in the prevention of onset of allergic disease: a 15-year prospective study. Clin Pediatr (Phila) 1982;21:486-491. [PubMed: 7200847]

Habito RC, Montalto J, Leslie E, Ball MJ. Effects of replacing meat with soyabean in the diet on sex hormone concentrations in healthy adult males. Br J Nutr 2000;84:557-563. [PubMed: 11103227]

Hakkak R, Korourian S, Shelnutt SR, Lensing S, Ronis MJ, Badger TM. Diets containing whey proteins or soy protein isolate protect against 7,12-dimethylbenz(a)anthracene-induced mammary tumors in female rats. Cancer Epidemiol Biomarkers Prev 2000;9:113-117. [PubMed: 10667471]

Hall RT, Callenbach JC, Sheehan MB, Hall FK, Thibeault DW, Kurth CG, Bowen SK. Comparison of calcium- and phosphorus-supplemented soy isolate formula with whey-predominant premature formula in very low birth weight infants. J Pediatr Gastroenterol Nutr 1984;3:571-576. [PubMed: 6332895]

Halpern SR, Sellars WA, Johnson RB, Anderson DW, Saperstein S, Reisch JS. Development of childhood allergy in infants fed breast, soy, or cow milk. J Allergy Clin Immunol 1973;51:139-151. [PubMed: 4739434]

Halpin TC, Byrne WJ, Ament ME. Colitis, persistent diarrhea, and soy protein intolerance. J Pediatr 1977;91:404-407. [PubMed: 561179]

Hargreaves DF, Potten CS, Harding C, Shaw LE, Morton MS, Roberts SA, Howell A, Bundred NJ. Twoweek dietary soy supplementation has an estrogenic effect on normal premenopausal breast. J Clin Endocrinol Metab 1999;84:4017-4024. [PubMed: 10566643]

Hillman LS. Bone mineral content in term infants fed human milk, cow milk-based formula, or soy-based formula. J Pediatr 1988;113:208-212. [PubMed: 3392640]

Hillman LS, Chow W, Salmons SS, Weaver E, Erickson M, Hansen J. Vitamin D metabolism, mineral homeostasis, and bone mineralization in term infants fed human milk, cow milk-based formula, or soy-based formula. J Pediatr 1988;112:864-874. [PubMed: 3373390]

Hoey L, Rowland IR, Lloyd AS, Clarke DB, Wiseman H. Influence of soya-based infant formula consumption on isoflavone and gut microflora metabolite concentrations in urine and on faecal 
microflora composition and metabolic activity in infants and children. Br J Nutr 2004;91:607-616. [PubMed: 15035688]

Huggett AC, Pridmore S, Malnoe A, Haschke F, Offord EA. Phytooestrogens in soy-based infant formula. Lancet 1997;350:815-816. [PubMed: 9298031]

Hughes CL, Liu G, Beall S, Foster WG, Davis V. Effects of genistein or soy milk during late gestation and lactation on adult uterine organization in the rat. Exp Biol Med (Maywood) 2004;229:108-117. [PubMed: 14709783]

Hutchins AM, Slavin JL, Lampe JW. Urinary isoflavonoid phytoestrogen and lignan excretion after consumption of fermented and unfermented soy products. J Am Diet Assoc 1995;95:545-551. [PubMed: 7722188]

ILSI. Safety assessment and potential health benefits of food components based on selected scientific criteria. ILSI North America Technical Committee on Food Components for Health Promotion. Crit Rev Food Sci Nutr 1999;39:203-316. [PubMed: 10367185]

Irvine CH, Fitzpatrick MG, Alexander SL. Phytoestrogens in soy-based infant foods: concentrations, daily intake, and possible biological effects. Proc Soc Exp Biol Med 1998a;217:247-253. [PubMed: 9492332]

Irvine $\mathrm{CH}$, Shand N, Fitzpatrick MG, Alexander SL. Daily intake and urinary excretion of genistein and daidzein by infants fed soy- or dairy-based infant formulas. Am J Clin Nutr 1998b;68:1462S-1465S. [PubMed: 9848517]

Iyngkaran N, Yadav M, Looi LM, Boey CG, Lam KL, Balabaskaran S, Puthucheary SD. Effect of soy protein on the small bowel mucosa of young infants recovering from acute gastroenteritis. J Pediatr Gastroenterol Nutr 1988;7:68-75. [PubMed: 3335989]

Jabbar MA, Larrea J, Shaw RA. Abnormal thyroid function tests in infants with congenital hypothyroidism: the influence of soy-based formula. J Am Coll Nutr 1997;16:280-282. [PubMed: 9176836]

Jakes RW, Alexander L, Duffy SW, Leong J, Chen LH, Lee WH. Dietary intake of soybean protein and menstrual cycle length in pre-menopausal Singapore Chinese women. Public Health Nutr 2001;4:191-196. [PubMed: 11299091]

Jenkins HR, Pincott JR, Soothill JF, Milla PJ, Harries JT. Food allergy: the major cause of infantile colitis. Arch Dis Child 1984;59:326-329. [PubMed: 6721558]

Joannou GE, Kelly GE, Reeder AY, Waring M, Nelson C. A urinary profile study of dietary phytoestrogens. The identification and mode of metabolism of new isoflavonoids. J Steroid Biochem Mol Biol 1995;54:167-184. [PubMed: 7662591]

Jung AL, Carr SL. A soy protein formula and a milk-based formula. A comparative evaluation in milktolerant infants showed no significant nutritional differences. Clin Pediatr (Phila) 1977;16:982-985. [PubMed: 578789]

Kay JL, Daeschner CW Jr, Desmond MM. Evaluation of infants fed soybean and evaporated milk formulae from birth to three months. A comparison of weight, length, hemoglobin, hematocrit, and plasma biochemical values. Am J Dis Child 1960;100:264-276. [PubMed: 14404995]

Kelly GE, Joannou GE, Reeder AY, Nelson C, Waring MA. The variable metabolic response to dietary isoflavones in humans. Proc Soc Exp Biol Med 1995;208:40-43. [PubMed: 7892293]

King RA, Mano MM, Head RJ. Assessment of isoflavonoid concentrations in Australian bovine milk samples. J Dairy Res 1998;65:479-489. [PubMed: 9718497]

Kjellman NI, Johansson SG. Soy versus cow's milk in infants with a biparental history of atopic disease: development of atopic disease and immunoglobulins from birth to 4 years of age. Clin Allergy 1979;9:347-358. [PubMed: 573185]

Klemola T, Kalimo K, Poussa T, Juntunen-Backman K, Korpela R, Valovirta E, Vanto T. Feeding a soy formula to children with cow's milk allergy: the development of immunoglobulin E-mediated allergy to soy and peanuts. Pediatr Allergy Immunol 2005;16:641-646. [PubMed: 16343085]

Klemola T, Vanto T, Juntunen-Backman K, Kalimo K, Korpela R, Varjonen E. Allergy to soy formula and to extensively hydrolyzed whey formula in infants with cow's milk allergy: a prospective, randomized study with a follow-up to the age of 2 years. J Pediatr 2002;140:219-224. [PubMed: 11865274] 
Knight DC, Eden JA, Huang JL, Waring MA, Irvine CH, Fitzpatrick MG, Alexander SL. Isoflavone content of infant foods and formulas. J Paediatr Child Health 1998;34:135-138. [PubMed: 9588635]

Köhler L, Meeuwisse G, Mortensson W. Food intake and growth of infants between six and twenty-six weeks of age on breast milk, cow's milk formula, or soy formula. Acta Paediatr Scand 1984;73:40_ 48. [PubMed: 6702448]

Kulkarni PB, Dorand RD, Bridger WM, Payne JH 3rd, Montiel DC, Hill JG. Rickets in premature infants fed different formulas. South Med J 1984;77:13-16. 20. [PubMed: 6364370]

Kulkarni PB, Hall RT, Rhodes PG, Sheehan MB, Callenbach JC, Germann DR, Abramson SJ. Rickets in very low-birth-weight infants. J Pediatr 1980;96:249-252. [PubMed: 7351588]

Kurzer MS, Xu X. Dietary phytoestrogens. Annu Rev Nutr 1997;17:353-381. [PubMed: 9240932]

Lack G, Fox D, Northstone K, Golding J. Factors associated with the development of peanut allergy in childhood. N Engl J Med 2003;348:977-985. [PubMed: 12637607]

Lasekan JB, Ostrom KM, Jacobs JR, Blatter MM, Ndife LI, Gooch WM 3rd, Cho S. Growth of newborn, term infants fed soy formulas for 1 year. Clin Pediatr (Phila) 1999;38:563-571. [PubMed: 10544862]

Lephart ED, Adlercreutz H, Lund TD. Dietary soy phytoestrogen effects on brain structure and aromatase in Long-Evans rats. Neuroreport 2001;12:3451-3455. [PubMed: 11733689]

Lephart ED, West TW, Weber KS, Rhees RW, Setchell KD, Adlercreutz H, Lund TD. Neurobehavioral effects of dietary soy phytoestrogens. Neurotoxicol Teratol 2002;24:5-16. [PubMed: 11836067]

Li DF, Nelssen JL, Reddy PG, Blecha F, Hancock JD, Allee GL, Goodband RD, Klemm RD. Transient hypersensitivity to soybean meal in the early-weaned pig. J Anim Sci 1990;68:1790-1799. [PubMed: 2384373]

Li L-H, Donald JM, Golub MS. Review on testicular development, structure, function, and regulation in common marmoset. Birth Defects Res B Dev Reprod Toxicol 2005;74:450-469. [PubMed: 16193499]

Lothe L, Lindberg T, Jakobsson I. Cow's milk formula as a cause of infantile colic: a double-blind study. Pediatrics 1982;70:7-10. [PubMed: 7088636]

Lu LJ, Anderson KE. Sex and long-term soy diets affect the metabolism and excretion of soy isoflavones in humans. Am J Clin Nutr 1998;68:1500S-1504S. [PubMed: 9848524]

Lu LJ, Anderson KE, Grady JJ, Kohen F, Nagamani M. Decreased ovarian hormones during a soya diet: implications for breast cancer prevention. Cancer Res 2000a;60:4112-4121. [PubMed: 10945618]

Lu LJ, Anderson KE, Grady JJ, Nagamani M. Effects of soya consumption for one month on steroid hormones in premenopausal women: implications for breast cancer risk reduction. Cancer Epidemiol Biomarkers Prev 1996;5:63-70. [PubMed: 8770469]

Lu LJ, Anderson KE, Grady JJ, Nagamani M. Effects of an isoflavone-free soy diet on ovarian hormones in premenopausal women. J Clin Endocrinol Metab 2001;86:3045-3052. [PubMed: 11443166]

Lu LJ, Cree M, Josyula S, Nagamani M, Grady JJ, Anderson KE. Increased urinary excretion of 2hydroxyestrone but not 16alpha-hydroxyestrone in premenopausal women during a soya diet containing isoflavones. Cancer Res 2000b;60:1299-1305. [PubMed: 10728690]

Lu LJ, Grady JJ, Marshall MV, Ramanujam VM, Anderson KE. Altered time course of urinary daidzein and genistein excretion during chronic soya diet in healthy male subjects. Nutr Cancer 1995;24:311323. [PubMed: 8610050]

Lund TD, Lephart ED. Dietary soy phytoestrogens produce anxiolytic effects in the elevated plus-maze. Brain Res 2001;913:180-184. [PubMed: 11549384]

Lund TD, Rhees RW, Setchell KD, Lephart ED. Altered sexually dimorphic nucleus of the preoptic area (SDN-POA) volume in adult Long-Evans rats by dietary soy phytoestrogens. Brain Res 2001a; 914:92-99. [PubMed: 11578601]

Lund TD, West TW, Tian LY, Bu LH, Simmons DL, Setchell KD, Adlercreutz H, Lephart ED. Visual spatial memory is enhanced in female rats (but inhibited in males) by dietary soy phytoestrogens. BMC Neurosci 2001b;2:20. [PubMed: 11801187]

MAFF. Levels of plant oestrogens in the diets of infants and toddlers. Reading, UK: The University of Reading; 1998a. 
MAFF. Plant oestrogens in soya-based infant formulae. London, UK: Ministry of Agriculture, Fisheries, and Food; 1998b. Available at http://archive.food.gov.uk/maff/archive/food/infsheet/1998/no167/167phy.htm.

Makela S, Santti R, Martikainen P, Nienstedt W, Paranko J. The influence of steroidal and nonsteroidal estrogens on the 5 alpha-reduction of testosterone by the ventral prostate of the rat. J Steroid Biochem 1990;35:249-256. [PubMed: 2308339]

Mäkelä S, Santti R, Salo L, McLachlan JA. Phytoestrogens are partial estrogen agonists in the adult male mouse. Environ Health Perspect 1995a;103:123-127.

Mäkelä SI, Pylkkanen LH, Santti RS, Adlercreutz H. Dietary soybean may be antiestrogenic in male mice. J Nutr 1995b;125:437-445.

Malloy MH, Berendes H. Does breast-feeding influence intelligence quotients at 9 and 10 years of age? Early Hum Dev 1998;50:209-217. [PubMed: 9483393]

Martini MC, Dancisak BB, Haggans CJ, Thomas W, Slavin JL. Effects of soy intake on sex hormone metabolism in premenopausal women. Nutr Cancer 1999;34:133-139. [PubMed: 10578479]

Maskarinec G, Franke AA, Williams AE, Hebshi S, Oshiro C, Murphy S, Stanczyk FZ. Effects of a 2year randomized soy intervention on sex hormone levels in premenopausal women. Cancer Epidemiol Biomarkers Prev 2004a;13:1736-1744. [PubMed: 15533901]

Maskarinec G, Takata Y, Franke AA, Williams AE, Murphy SP. A 2-year soy intervention in premenopausal women does not change mammographic densities. J Nutr 2004b;134:3089-3094. [PubMed: 15514280]

Masutomi N, Shibutani M, Takagi H, Uneyama C, Hirose M. Dietary influence on the impact of ethinylestradiol-induced alterations in the endocrine/reproductive system with perinatal maternal exposure. Reprod Toxicol 2004;18:23-33. [PubMed: 15013061]

May CD, Fomon SJ, Remigio L. Immunologic consequences of feeding infants with cow milk and soy products. Acta Paediatr Scand 1982;71:43-51. [PubMed: 6890291]

McClain RM. Mechanistic considerations for the relevance of animal data on thyroid neoplasia to human risk assessment. Mutat Res 1995;333:131-142. [PubMed: 8538620]

McDonald PJ, Goldblum RM, Van Sickle GJ, Powell GK. Food protein-induced enterocolitis: altered antibody response to ingested antigen. Pediatr Res 1984;18:751-755. [PubMed: 6540862]

McMichael-Phillips DF, Harding C, Morton M, Roberts SA, Howell A, Potten CS, Bundred NJ. Effects of soy-protein supplementation on epithelial proliferation in the histologically normal human breast. Am J Clin Nutr 1998;68:1431S-1435S. [PubMed: 9848512]

McVey MJ, Cooke GM, Curran IH. Altered testicular microsomal steroidogenic enzyme activities in rats with lifetime exposure to soy isoflavones. J Steroid Biochem Mol Biol 2004a;92:435-446. [PubMed: 15698548]

McVey MJ, Cooke GM, Curran IH. Increased serum and testicular androgen levels in F1 rats with lifetime exposure to soy isoflavones. Reprod Toxicol 2004b;18:677-685. [PubMed: 15219630]

Mimouni F, Campaigne B, Neylan M, Tsang RC. Bone mineralization in the first year of life in infants fed human milk, cow-milk formula, or soy-based formula. J Pediatr 1993;122:348-354. [PubMed: 8441086]

Miskelly FG, Burr ML, Vaughan-Williams E, Fehily AM, Butland BK, Merrett TG. Infant feeding and allergy. Arch Dis Child 1988;63:388-393. [PubMed: 3365008]

Mitchell JH, Cawood E, Kinniburgh D, Provan A, Collins AR, Irvine DS. Effect of a phytoestrogen food supplement on reproductive health in normal males. Clin Sci (Lond) 2001;100:613-618. [PubMed: 11352776]

Moore WJ, Midwinter RE, Morris AF, Colley JR, Soothill JF. Infant feeding and subsequent risk of atopic eczema. Arch Dis Child 1985;60:722-726. [PubMed: 3899021]

Mortimer EZ. Anaphylaxis following ingestion of soybean. J Pediatr 1961;58:90-92. [PubMed: 13772958]

Mousavi SM, Tavakoli N, Mardan F. Risk factors for goiter in primary school girls in Qom city of Iran. Eur J Clin Nutr 2005;23:23.

Munro IC, Harwood M, Hlywka JJ, Stephen AM, Doull J, Flamm WG, Adlercreutz H. Soy isoflavones: a safety review. Nutr Rev 2003;61:1-33. [PubMed: 12638461] 
Murkies AL, Lombard C, Strauss BJ, Wilcox G, Burger HG, Morton MS. Dietary flour supplementation decreases post-menopausal hot flushes: effect of soy and wheat. Maturitas 1995;21:189-195. [PubMed: 7616867]

Murphy PA, Song T, Buseman G, Barua K. Isoflavones in soy-based infant formulas. J Agric Food Chem 1997;45:4635-4638.

Nagata C, Inaba S, Kawakami N, Kakizoe T, Shimizu H. Inverse association of soy product intake with serum androgen and estrogen concentrations in Japanese men. Nutr Cancer 2000;36:14-18. [PubMed: 10798211]

Nagata C, Kabuto M, Kurisu Y, Shimizu H. Decreased serum estradiol concentration associated with high dietary intake of soy products in premenopausal Japanese women. Nutr Cancer 1997;29:228233. [PubMed: 9457744]

Nagata C, Takatsuka N, Inaba S, Kawakami N, Shimizu H. Effect of soy milk consumption on serum estrogen concentrations in premenopausal Japanese women. J Natl Cancer Inst 1998;90:1830-1835. [PubMed: 9839524]

Nagata C, Takatsuka N, Shimizu H, Hayashi H, Akamatsu T, Murase K. Effect of soy milk consumption on serum estrogen and androgen concentrations in Japanese men. Cancer Epidemiol Biomarkers Prev 2001;10:179-184. [PubMed: 11303585]

Nakai M, Black M, Jeffery EH, Bahr JM. Dietary soy protein and isoflavones: no effect on the reproductive tract and minimal positive effect on bone resorption in the intact female Fischer 344 rat. Food Chem Toxicol 2005;43:945-949. [PubMed: 15811574]

Naude SP, Prinsloo JG, Haupt CE. Comparison between a humanized cow's milk and a soy product for premature infants. S Afr Med J 1979;55:982-986. [PubMed: 572996]

Nguyenle T, Wang E, Cheung AP. An investigation on the extraction and concentration of isoflavones in soy-based products. J Pharm Biomed Anal 1995;14:221-232. [PubMed: 8833985]

North K, Golding J. A maternal vegetarian diet in pregnancy is associated with hypospadias. The ALSPAC Study Team. Avon Longitudinal Study of Pregnancy and Childhood. BJU Int 2000;85:107-113. [PubMed: 10619956]

Odum J, Tinwell H, Jones K, Van Miller JP, Joiner RL, Tobin G, Kawasaki H, Deghenghi R, Ashby J. Effect of rodent diets on the sexual development of the rat. Toxicol Sci 2001;61:115-127. [PubMed: 11294982]

Odze RD, Bines J, Leichtner AM, Goldman H, Antonioli DA. Allergic proctocolitis in infants: a prospective clinicopathologic biopsy study. Hum Pathol 1993;24:668-674. [PubMed: 8505043]

Oliveira, V.; Prell, M.; Smallwood, D.; Frazao, E. Infant formula prices and availability-final report to congress. United States Department of Agriculture Economic Research Service; 2001. Available at http://www.ers.usda.gov/publications/efan02001/efan02001.pdf.

Ostrom KM, Cordle CT, Schaller JP, Winship TR, Thomas DJ, Jacobs JR, Blatter MM, Cho S, Gooch WM 3rd, Granoff DM, Faden H, Pickering LK. Immune status of infants fed soy-based formulas with or without added nucleotides for 1 year: part 1: vaccine responses, and morbidity. J Pediatr Gastroenterol Nutr 2002;34:137-144. [PubMed: 11840030]

Ostrom KM, Jacobs JR, Merritt RJ, Murray RD. Decreased regurgitation with a soy formula containing added soy fiber. Clin Pediatr 2006;45:29-36.

Patisaul HB, Dindo M, Whitten PL, Young LJ. Soy isoflavone supplements antagonize reproductive behavior and estrogen receptor alpha- and beta-dependent gene expression in the brain. Endocrinology 2001;142:2946-2952. [PubMed: 11416015]

Patisaul HB, Luskin JR, Wilson ME. A soy supplement and tamoxifen inhibit sexual behavior in female rats. Horm Behav 2004;45:270-277. [PubMed: 15053943]

Perkkio M, Savilahti E, Kuitunen P. Morphometric and immunohistochemical study of jejunal biopsies from children with intestinal soy allergy. Eur J Pediatr 1981;137:63-69. [PubMed: 7196837]

Piotrowska KK, Woclawek-Potocka I, Bah MM, Piskula MK, Pilawski W, Bober A, Skarzynski DJ. Phytoestrogens and their metabolites inhibit the sensitivity of the bovine corpus luteum to luteotropic factors. J Reprod Dev 2006;52:33-41. [PubMed: 16276041]

Poley JR, Klein AW. Scanning electron microscopy of soy protein-induced damage of small bowel mucosa in infants. J Pediatr Gastroenterol Nutr 1983;2:271-287. [PubMed: 6683755] 
Powell GK. Milk- and soy-induced enterocolitis of infancy: clinical features and standardization of challenge. J Pediatr 1978;93:553-560. [PubMed: 568171]

Price KR, Fenwick GR. Naturally occurring oestrogens in foods-A review. Food Addit Contam 1985;2:73-106. [PubMed: 4018320]

Pumford SL, Morton MM, Turkes A, Griffiths K. Determination of the isoflavonoids genistein and daidzein in biological samples by gas chromatography-mass spectrometry. Ann Clin Biochem 2002;39:281-292. [PubMed: 12038603]

Robertson KM, O'Donnell L, Simpson ER, Jones ME. The phenotype of the aromatase knockout mouse reveals dietary phytoestrogens impact significantly on testis function. Endocrinology 2002;143:2913-2921. [PubMed: 12130556]

Ronis MJ, Chen Y, Badeaux J, Laurenzana E, Badger TM. Soy protein isolate induces CYP3A1 and CYP3A2 in prepubertal rats. Exp Biol Med 2006;231:60-69.

Ronis MJ, Chen Y, Jo CH, Simpson P, Badger TM. Diets containing soy protein isolate increase hepatic CYP3A expression and inducibility in weanling male rats exposed during early development. J Nutr 2004;134:3270-3276. [PubMed: 15570024]

Ronis MJ, Rowlands JC, Hakkak R, Badger TM. Altered expression and glucocorticoid-inducibility of hepatic CYP3A and CYP2B enzymes in male rats fed diets containing soy protein isolate. J Nutr 1999;129:1958-1965. [PubMed: 10539769]

Rowland IR, Wiseman H, Sanders TA, Adlercreutz H, Bowey EA. Interindividual variation in metabolism of soy isoflavones and lignans: influence of habitual diet on equol production by the gut microflora. Nutr Cancer 2000;36:27-32. [PubMed: 10798213]

Rowlands JC, Hakkak R, Ronis MJ, Badger TM. Altered mammary gland differentiation and progesterone receptor expression in rats fed soy and whey proteins. Toxicol Sci 2002;70:40-45. [PubMed: 12388833]

Rowlands JC, He L, Hakkak R, Ronis MJ, Badger TM. Soy and whey proteins downregulate DMBAinduced liver and mammary gland CYP1 expression in female rats. J Nutr 2001;131:3281-3287. [PubMed: 11739881]

Rozman KK, Kesner JS, Calafat AM, Jeffery EH, Culty M, Bhatia J, Etzel RA, Marty S, Hansen DK, Flaws JA, Chambers C, Thomas JA, Hoyer PB, Umbach D. NTP-CERHR Expert Panel Report on the reproductive and developmental toxicity of soy formula. Birth Defects Res (Part B). in press

Sellars WA, Halpern SR, Johnson RB, Anderson DW Jr, Saperstein S, Shannon BS Jr. New growth charts: soy, cow, and breast milk comparison. Ann Allergy 1971;29:126-134. [PubMed: 5172767]

Setchell K, Brown NM, Lydeking-Olsen E. The clinical importance of the metabolite equol-a clue to the effectiveness of soy and its isoflavones. J Nutr 2002;132:3577-3584. [PubMed: 12468591]

Setchell KD. Phytoestrogens: the biochemistry, physiology, and implications for human health of soy isoflavones. Am J Clin Nutr 1998;68:1333S-1346S. [PubMed: 9848496]

Setchell KD, Borriello SP, Hulme P, Kirk DN, Axelson M. Nonsteroidal estrogens of dietary origin: possible roles in hormone-dependent disease. Am J Clin Nutr 1984;40:569-578. [PubMed: 6383008]

Setchell KD, Brown NM, Desai P, Zimmer-Nechemias L, Wolfe BE, Brashear WT, Kirschner AS, Cassidy A, Heubi JE. Bioavailability of pure isoflavones in healthy humans and analysis of commercial soy isoflavone supplements. J Nutr 2001;131:1362S-1375S. [PubMed: 11285356]

Setchell KD, Faughnan MS, Avades T, Zimmer-Nechemias L, Brown NM, Wolfe BE, Brashear WT, Desai P, Oldfield MF, Botting NP, Cassidy A. Comparing the pharmacokinetics of daidzein and genistein with the use of 13C-labeled tracers in premenopausal women. Am J Clin Nutr 2003;77:411-419. [PubMed: 12540402]

Setchell KD, Zimmer-Nechemias L, Cai J, Heubi JE. Exposure of infants to phyto-oestrogens from soybased infant formula. Lancet 1997;350:23-27. [PubMed: 9217716]

Setchell KD, Zimmer-Nechemias L, Cai J, Heubi JE. Isoflavone content of infant formulas and the metabolic fate of these phytoestrogens in early life. Am J Clin Nutr 1998;68:1453S-1461S. [PubMed: 9848516]

Sfakianos J, Coward L, Kirk M, Barnes S. Intestinal uptake and biliary excretion of the isoflavone genistein in rats. J Nutr 1997;127:1260-1268. [PubMed: 9202077] 
Sharma OP, Adlercreutz H, Strandberg JD, Zirkin BR, Coffey DS, Ewing LL. Soy of dietary source plays a preventive role against the pathogenesis of prostatitis in rats. J Steroid Biochem Mol Biol 1992;43:557-564. [PubMed: 1419891]

Sharpe RM, Martin B, Morris K, Greig I, McKinnell C, McNeilly AS, Walker M, Setchell KD, Cassidy A, Ingram D, Sanders K, Kolybaba M, Lopez D, Fitzpatrick M, Fort P, Moses N, Fasano M, Goldberg T, Lifshitz F, Chang HC, Doerge DR, Bingham S, Setchell K, Herman-Giddens ME, Slora EJ, Wasserman RC, Bourdony CJ, Bhapkar MV, Koch GG, Hasemeier CM. Infant feeding with soy formula milk: effects on the testis and on blood testosterone levels in marmoset monkeys during the period of neonatal testicular activity. Hum Reprod 2002;17:1692-1703. [PubMed: 12093826]

Shenai JP, Jhaveri BM, Reynolds JW, Huston RK, Babson SG. Nutritional balance studies in very lowbirth-weight infants: role of soy formula. Pediatrics 1981;67:631-637. [PubMed: 7196014]

Shepard T. Soybean goiter. New Engl J Med 1960;262:1099-1103.

Shu XO, Jin F, Dai Q, Wen W, Potter JD, Kushi LH, Ruan Z, Gao YT, Zheng W. Soyfood intake during adolescence and subsequent risk of breast cancer among Chinese women. Cancer Epidemiol Biomarkers Prev 2001;10:483-488. [PubMed: 11352858]

Simmen RC, Eason RR, Till SR, Chatman L Jr, Velarde MC, Geng Y, Korourian S, Badger TM. Inhibition of NMU-induced mammary tumorigenesis by dietary soy. Cancer Lett 2005;224:45-52. [PubMed: 15911100]

Stauffer BL, Konhilas JP, Luczak ED, Leinwand LA. Soy diet worsens heart disease in mice. J Clin Invest 2006;116:209-216. [PubMed: 16395406]

Steer TE, Johnson IT, Gee JM, Gibson GR. Metabolism of the soybean isoflavone glycoside genistein in vitro by human gut bacteria and the effect of prebiotics. Br J Nutr 2003;90:635-642. [PubMed: 13129470]

Steichen JJ, Tsang RC. Bone mineralization and growth in term infants fed soy-based or cow milk-based formula. J Pediatr 1987;110:687-692. [PubMed: 3572619]

Stettler N, Stallings VA, Troxel AB, Zhao J, Schinnar R, Nelson SE, Ziegler EE, Strom BL. Weight gain in the first week of life and overweight in adulthood: a cohort study of European American subjects fed infant formula. Circulation 2005;111:1897-1903. [PubMed: 15837942]

Strom BL, Schinnar R, Ziegler EE, Barnhart KT, Sammel MD, Macones GA, Stallings VA, Drulis JM, Nelson SE, Hanson SA. Exposure to soy-based formula in infancy and endocrinological and reproductive outcomes in young adulthood. JAMA 2001;286:807-814. [PubMed: 11497534]

Tan KAL, Walker M, Morris K, Greig I, Mason JI, Sharpe RM. Infant feeding with soy formula milk: effects on puberty progression, reproductive function and testicular cell numbers in marmoset monkeys in adulthood. Hum Reprod 2006;21:896-904. [PubMed: 16476680]

Tansey G, Hughes CL Jr, Cline JM, Krummer A, Walmer DK, Schmoltzer S. Effects of dietary soybean estrogens on the reproductive tract in female rats. Proc Soc Exp Biol Med 1998;217:340-344. [PubMed: 9492345]

Taylor H, Quintero EM, Iacopino AM, Lephart ED. Phytoestrogens alter hypothalamic calbindin-D28k levels during prenatal development. Brain Res Dev Brain Res 1999;114:277-281.

Teichberg S, Isolauri E, Wapnir RA, Roberts B, Lifshitz F. Development of the neonatal rat small intestinal barrier to nonspecific macromolecular absorption: effect of early weaning to artificial diets. Pediatr Res 1990;28:31-37. [PubMed: 2377394]

Teichberg S, Wapnir RA, Moyse J, Lifshitz F. Development of the neonatal rat small intestinal barrier to nonspecific macromolecular absorption. II. Role of dietary corticosterone. Pediatr Res 1992;32:50-57. [PubMed: 1635845]

Thigpen JE, Li LA, Richter CB, Lebetkin EH, Jameson CW. The mouse bioassay for the detection of estrogenic activity in rodent diets: II. Comparative estrogenic activity of purified, certified and standard open and closed formula rodent diets. Lab Anim Sci 1987;37:602-605. [PubMed: 3695394]

Thigpen JE, Setchell KD, Goelz MF, Forsythe DB. The phytoestrogen content of rodent diets. Environ Health Perspect 1999;107:A182-A183. [PubMed: 10383244]

Tuohy PG. Soy infant formula and phytoestrogens. J Paediatr Child Health 2003;39:401-405. [PubMed: 12919490] 
UK Committee on Toxicity. Phytoestrogens and health. London, UK: Committee on Toxicity of Chemicals in Food, Consumer Products and the Environment; 2003. http://www.food.gov.uk/multimedia/pdfs/phytoreport0503.

Unfer V, Casini ML, Costabile L, Mignosa M, Gerli S, Di Renzo GC. Endometrial effects of long-term treatment with phytoestrogens: a randomized, double-blind, placebo-controlled study. Fertil Steril 2004;82:145-148. [PubMed: 15237003]

USEPA. Recommendations and documentation of biological values for use in risk assessment. Cincinnati, $\mathrm{OH}$ : Environmental Criteria and Assessment Office, Office of Health and Environmental Assessment. Office of Research and Development. U.S. Environmental Protection Agency; 1988. EPA/600/6-87/008, NTIS PB88-179874/AS. Report nr EPA/600/6-87/008, NTIS PB88-179874/ AS.

USDA. Iron-fortified infant formulas that do not require medical statements in the child nutrition programs. United States Department of Agriculture; 2002a. Available at http://www.cde.ca.gov/nsd/npm/mb021300222802718att.pdf.

USDA. USDA-Iowa State University database on the isoflavone content of food, Release 1.3. United States Department of Agriculture and Iowa State University; 2002b. Available at http://www.nal.usda.gov/fnic/foodcomp/Data/isoflav/isoflav.html.

Venkataraman PS, Luhar H, Neylan MJ. Bone mineral metabolism in full-term infants fed human milk, cow milk-based, and soy-based formulas. Am J Dis Child 1992;146:1302-1305. [PubMed: 1415066]

Wade MG, Lee A, McMahon A, Cooke G, Curran I. The influence of dietary isoflavone on the uterotrophic response in juvenile rats. Food Chem Toxicol 2003;41:1517-1525. [PubMed: 12963004]

Watanabe S, Terashima K, Sato Y, Arai S, Eboshida A. Effects of isoflavone supplement on healthy women. Biofactors 2000;12:233-241. [PubMed: 11216491]

Weber KS, Jacobson NA, Setchell KD, Lephart ED. Brain aromatase and 5alpha-reductase, regulatory behaviors and testosterone levels in adult rats on phytoestrogen diets. Proc Soc Exp Biol Med 1999;221:131-135. [PubMed: 10352124]

Weber KS, Setchell KD, Lephart ED. Maternal and perinatal brain aromatase: effects of dietary soy phytoestrogens. Brain Res Dev Brain Res 2001a;126:217-221.

Weber KS, Setchell KDR, Stocco DM, Lephart ED. Dietary soy-phytoestrogens decrease testosterone levels and prostate weight without altering $\mathrm{LH}$, prostate 5alpha-reductase or testicular steroidogenic acute regulatory peptide levels in adult male Sprague-Dawley rats. J Endocrinol 2001b;170:591599. [PubMed: 11524239]

Whitington PF, Gibson R. Soy protein intolerance: Four patients with concomitant cow's milk intolerance. Pediatrics 1977;59:730-732. [PubMed: 558576]

Whitten PL, Patisaul HB. Cross-species and interassay comparisons of phytoestrogen action. Environ Health Perspect 2001;109:5-20. [PubMed: 11250801]

Wilcox G, Wahlqvist ML, Burger HG, Medley G. Oestrogenic effects of plant foods in postmenopausal women. BMJ 1990;301:905-906. [PubMed: 2124510]

Woclawek-Potocka I, Bah MM, Korzekwa A, Piskula MK, Wiczkowski W, Depta A, Skarzynski DJ. Soybean-derived phytoestrogens regulate prostaglandin secretion in endometrium during cattle estrous cycle and early pregnancy. Exp Biol Med 2005;230:189-199.

Wood CE, Kaplan JR, Stute P, Cline JM. Effects of soy on the mammary gland of premenopausal female monkeys. Fertil Steril 2006;85:1179-1186. [PubMed: 16616090]

Wood CE, Register TC, Anthony MS, Kock ND, Cline JM. Breast and uterine effects of soy isoflavones and conjugated equine estrogens in postmenopausal female monkeys. J Clin Endocrinol Metab 2004;89:3462-3468. [PubMed: 15240632]

Wu AH, Stanczyk FZ, Hendrich S, Murphy PA, Zhang C, Wan P, Pike MC. Effects of soy foods on ovarian function in premenopausal women. Br J Cancer 2000;82:1879-1886. [PubMed: 10839307]

Wu AH, Wan P, Hankin J, Tseng CC, Yu MC, Pike MC. Adolescent and adult soy intake and risk of breast cancer in Asian-Americans. Carcinogenesis 2002;23:1491-1496. [PubMed: 12189192] 
Xu X, Duncan AM, Merz BE, Kurzer MS. Effects of soy isoflavones on estrogen and phytoestrogen metabolism in premenopausal women. Cancer Epidemiol Biomarkers Prev 1998;7:1101-1108. [PubMed: 9865428]

Xu X, Wang HJ, Murphy PA, Cook L, Hendrich S. Daidzein is a more bioavailable soy milk isoflavone than is genistein in adult women. J Nutr 1994;124:825-832. [PubMed: 8207540]

Xu X, Wang HJ, Murphy PA, Hendrich S. Neither background diet nor type of soy food affects shortterm isoflavone bioavailability in women. J Nutr 2000;130:798-801. [PubMed: 10736332]

Yi MA, Son HM, Lee JS, Kwon CS, Lim JK, Yeo YK, Park YS, Kim JS. Regulation of male sex hormone levels by soy isoflavones in rats. Nutr Cancer 2002;42:206-210. [PubMed: 12416261]

Zhang Y, Wang GJ, Song TT, Murphy PA, Hendrich S. Urinary disposition of the soybean isoflavones daidzein, genistein and glycitein differs among humans with moderate fecal isoflavone degradation activity. J Nutr 1999;129:957-962. [PubMed: 10222386]

Zijlstra RT, Mies AM, McCracken BA, Odle J, Gaskins HR, Lien EL, Donovan SM. Short-term metabolic responses do not differ between neonatal piglets fed formulas containing hydrolyzed or intact soy proteins. J Nutr 1996;126:913-923. [PubMed: 8613895]

Zoppi G, Gerosa F, Pezzini A, Bassani N, Rizzotti P, Bellini P, Todeschini G, Zamboni G, Vazzoler G, Tridente G. Immunocompetence and dietary protein intake in early infancy. J Pediatr Gastroenterol Nutr 1982;1:175-182. [PubMed: 6985259]

Zoppi G, Mantovanelli F, Astolfi R, Gasparini R, Gobio Casali L, et al. Diet and antibody response to vaccinations in healthy infants. Lancet 1983;2:11-14. [PubMed: 6134885]

Zoppi G, Zamboni G, Bassani N, Vazzoler G. Gammaglobulin level and soy-protein intake in early infancy. Eur J Pediatr 1979;131:61-69. [PubMed: 86444] 
<smiles>O=c1c(-c2ccc(O)cc2)coc2cc(O)cc(O)c12</smiles>

Genistein<smiles>O=c1c(-c2ccc(O)cc2)coc2cc(O)ccc12</smiles>

Daidzein<smiles>COc1cc2c(=O)c(-c3ccc(O)cc3)coc2cc1O</smiles>

Glycitein

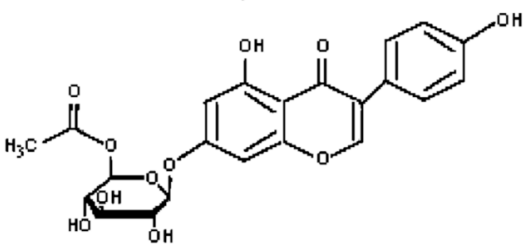

Acetylgenistin<smiles></smiles>

Acetyldaidzin<smiles>O=c1c(-c2ccc(O)cc2)coc2cc(OC3O[C@H](O)[C@@H](O)[C@@H](O)[C@H]3O)cc(O)c12</smiles>

Genistin (genistein glucoside)<smiles>O=c1c(-c2ccc(O)cc2)coc2cc(OC3[C@@H](O)[C@H](O)[C@H](O)[C@H]3O)ccc12</smiles>

Daidzin (daidzein glucoside)<smiles>COc1cc2c(=O)c(-c3ccc(O)cc3)coc2cc1O[C@H](O)[C@H](O)[C@H](O)C(O)O</smiles>

Glycitin (glycitein glucoside)<smiles></smiles>

Malonylgenistin<smiles>O=C(O)CC(=O)OC(O)[C@H](O)Oc1ccc2c(=O)c(-c3ccc(O)cc3)coc2c1</smiles>

Malonyldaidzin<smiles>COc1cc2c(=O)c(-c3ccc(O)cc3)coc2cc1O[C@H](O)[C@H](C)O</smiles>

Fig. 1.

Chemical structures of isoflavones found in soy formula. 

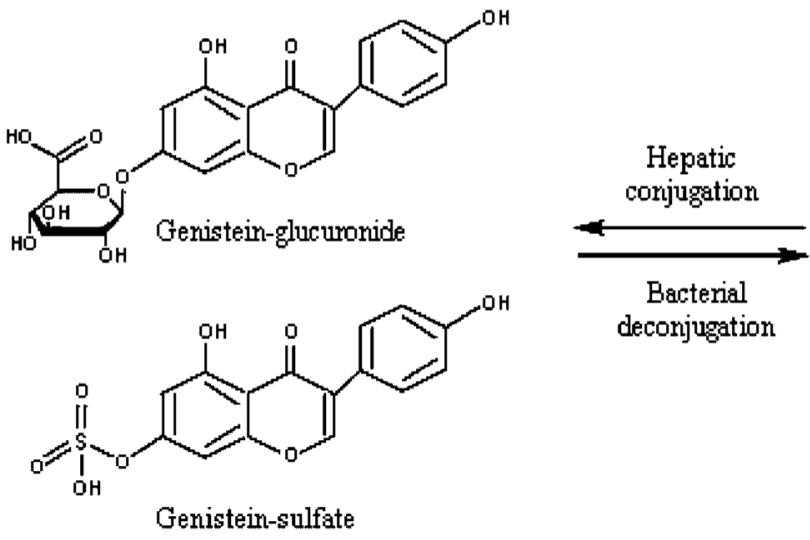
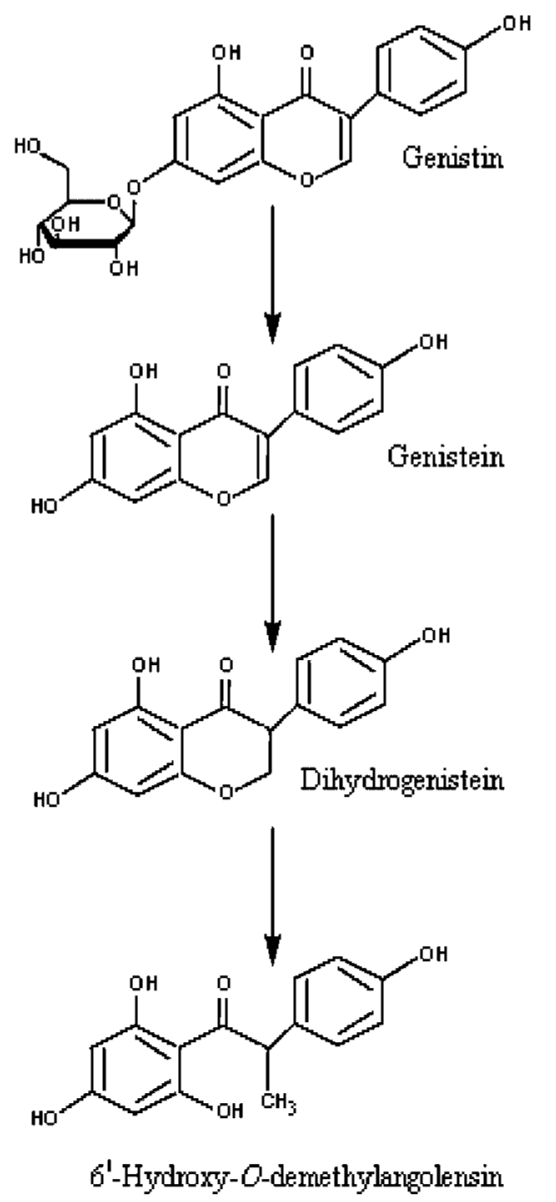

Fig. 2.

Metabolism of Genistein. Adapted from UK Committee on Toxicity (2003). 


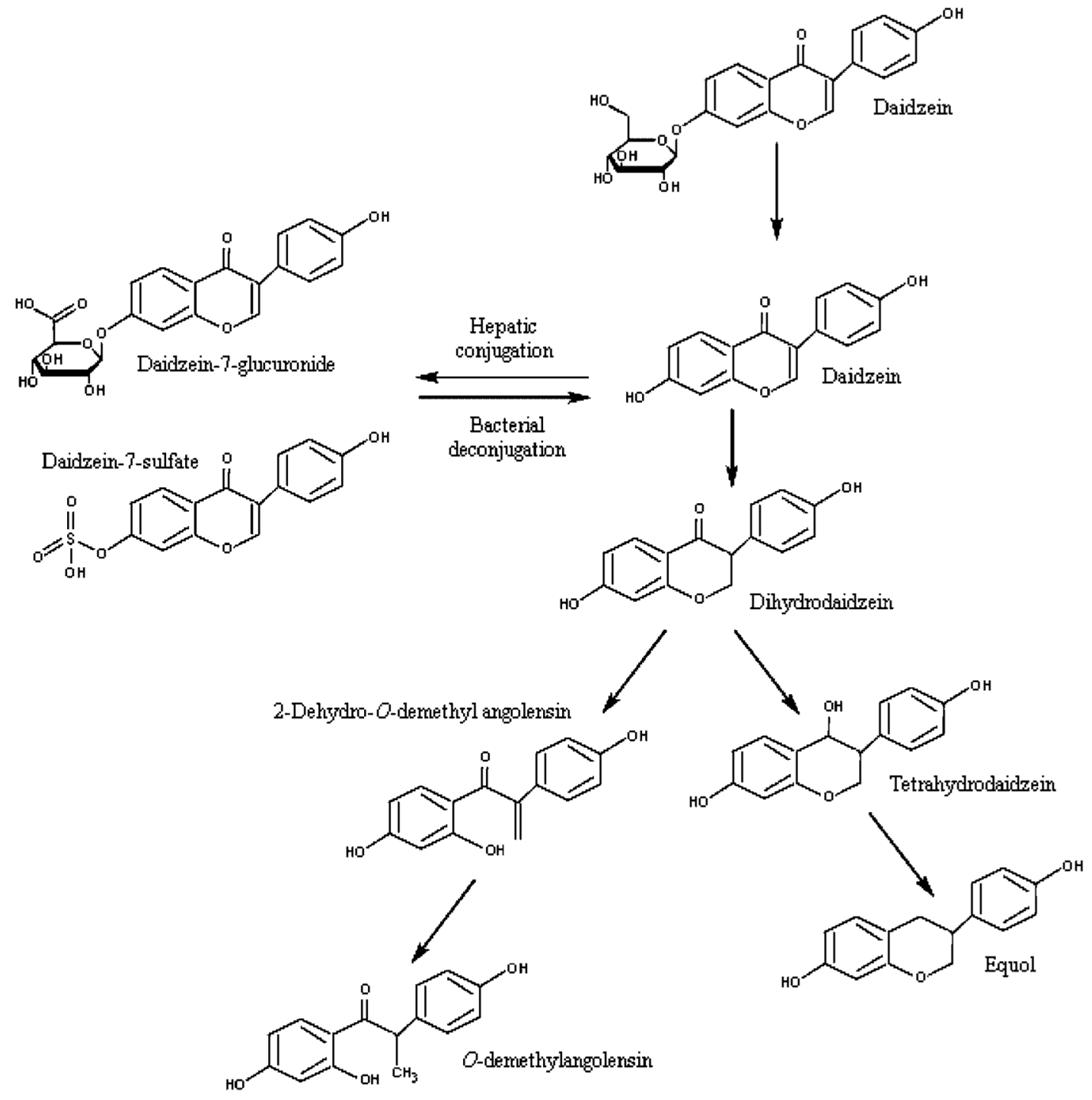

Fig. 3.

Metabolism of Daidzin. Adapted from UK Committee on Toxicity (2003) and Joannou et al. (1995). 

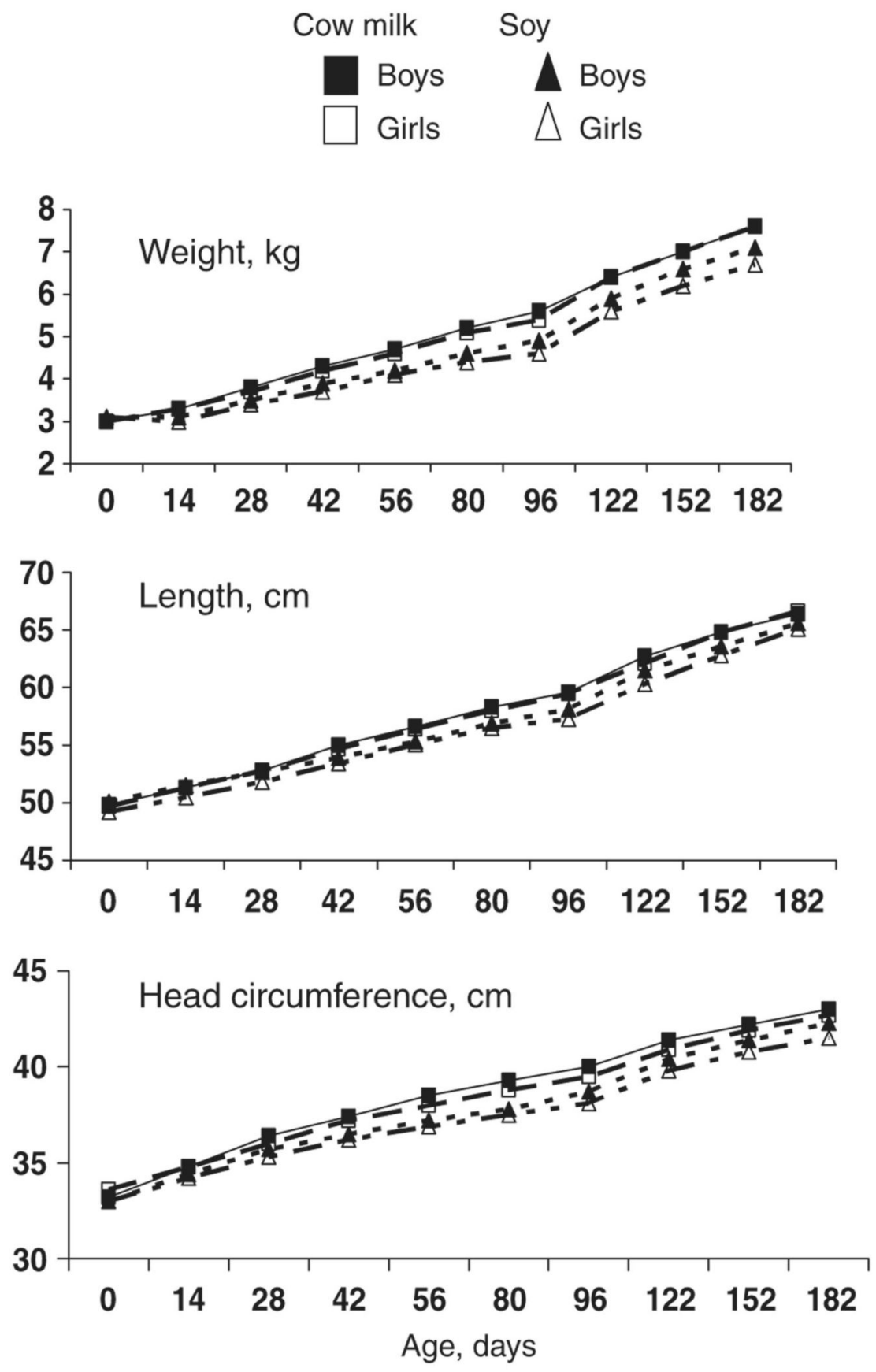

Fig. 4.

Growth parameters in children assigned to soy formula or cow-milk formula for 3 months. $\mathrm{n}$ =14-20 per data point, error bars omitted for clarity. From data presented by Cherry et al. (1968). 


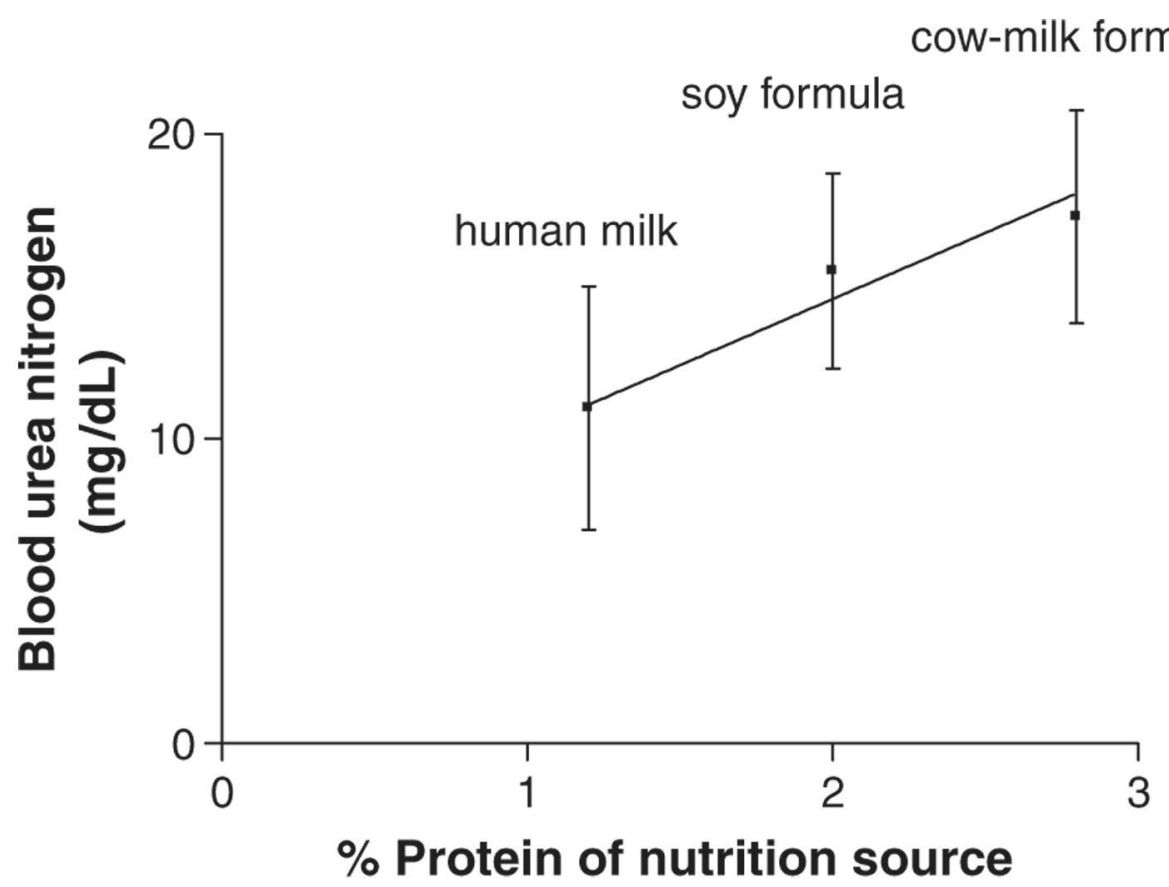

Fig. 5.

Infant blood urea nitrogen as a function of the protein content of food source. Human milk protein $1.2 \%$, soy formula protein $2.0 \%$, and cow-milk formula protein $2.8 \%$. Data expressed as mean \pm SD. From data presented by Dean (1973). 
Table 1

Primary Ingredients in Powdered Enfamil and Isomil Brand Soy Formulas ${ }^{a}$

\begin{tabular}{ll}
\hline Ingredient & Percentage [weight assumed] \\
\hline Corn syrup & $40.8-55$ \\
Soy protein isolate & $14.6-18$ \\
Vegetable oils & $21-28.6$ \\
Sugar & $10.2-11$ \\
\hline
\end{tabular}

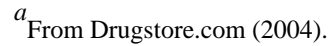

${ }^{b}$ Listed as sucrose in some formulations but not specified in others. 
Table 2

US Soy Formula Manufacturers and Brand Names ${ }^{a}$

\begin{tabular}{ll}
\hline Manufacturer & Brand Names \\
\hline Mead Johnson & Prosobee \\
& Gerber Soy \\
Ross & Isomil \\
Carnation & Alsoy \\
Wyeth-Ayerst & Nursoy \\
Wyeth (sold by PBM) & Variours store brands (e.g. CVS, K-mart, Kroger, Safeway, Target) \\
\hline
\end{tabular}

${ }^{a}$ Brand names are registered to their owners. From Oliveira et al. (2001) and USDA (2002a,b). 
Table 3

USDA-Iowa State University Survey of Soy Infant Formulas ${ }^{a}$

\begin{tabular}{|c|c|c|c|c|}
\hline \multirow[b]{2}{*}{ Formula Description } & \multicolumn{3}{|c|}{ Aglycone equivalents (after hydrolysis) } & \multirow[b]{2}{*}{ Confidence code ${ }^{c}$} \\
\hline & Isoflavone & $\begin{array}{l}\text { Mean (range) } \mathrm{mg} / \\
100 \mathrm{~g} \text { formula }\end{array}$ & Isoflavone $\%^{b}$ & \\
\hline \multirow{4}{*}{$\begin{array}{l}\text { ENFAMIL NEXT STEP, powder, not } \\
\text { reconstituted }\end{array}$} & Daidzein & $7.23(7.15-7.30)$ & 28.9 & $\mathrm{~b}$ \\
\hline & Genistein & $14.75(14.50-15.00)$ & 59.0 & $\mathrm{~b}$ \\
\hline & Glycitein & $3.00(2.95-3.05)$ & 12.0 & $\mathrm{~b}$ \\
\hline & Total isoflavone & $25.00(24.90-25.10)$ & & $\mathrm{b}$ \\
\hline \multirow{4}{*}{$\begin{array}{l}\text { MEAD JOHNSON, GERBER, } \\
\text { powder, not reconstituted }\end{array}$} & Daidzein & $8.08(6.50-9.65)$ & 32.2 & $\mathrm{~b}$ \\
\hline & Genistein & $13.90(12.80-15.00)$ & 55.4 & $\mathrm{~b}$ \\
\hline & Glycitein & $3.12(2.93-3.30)$ & 12.5 & $\mathrm{~b}$ \\
\hline & Total isoflavone & $25.09(22.23-27.95)$ & & $\mathrm{b}$ \\
\hline \multirow{3}{*}{$\begin{array}{l}\text { MEAD JOHNSON, PROSOBEE with } \\
\text { iron, liquid concentrate, not } \\
\text { reconstituted }\end{array}$} & Daidzein & 1.10 & 18.2 & $\mathrm{c}$ \\
\hline & Genistein & 2.22 & 36.8 & $\mathrm{c}$ \\
\hline & Total isoflavone & $6.03(3.32-8.75)$ & & $\mathrm{c}$ \\
\hline \multirow{4}{*}{$\begin{array}{l}\text { MEAD JOHNSON, PROSOBEE with } \\
\text { iron, powder, not reconstituted }\end{array}$} & Daidzein & $7.05(6.90-7.20)$ & 28.3 & $\mathrm{~b}$ \\
\hline & Genistein & $14.94(14.45-15.43)$ & 59.9 & $\mathrm{~b}$ \\
\hline & Glycitein & $2.95(2.83-3.07)$ & 11.8 & $\mathrm{~b}$ \\
\hline & Total isoflavone & $24.94(24.18-25.70)$ & & $\mathrm{b}$ \\
\hline \multirow{3}{*}{$\begin{array}{l}\text { MEAD JOHNSON, PROSOBEE with } \\
\text { iron, ready-to-feed }\end{array}$} & Daidzein & 1.71 & 44.0 & $\mathrm{c}$ \\
\hline & Genistein & 2.18 & 56.0 & $\mathrm{c}$ \\
\hline & Total isoflavone & 3.89 & & $\mathrm{c}$ \\
\hline \multirow{4}{*}{$\begin{array}{l}\text { ROSS, ISOMIL, with iron, powder, } \\
\text { not reconstituted }\end{array}$} & Daidzein & $6.03(6.03-6.03)$ & 28.7 & $\mathrm{~b}$ \\
\hline & Genistein & $12.23(11.43-13.03)$ & 58.3 & $\mathrm{~b}$ \\
\hline & Glycitein & $2.73(2.70-2.77)$ & 13.0 & $\mathrm{~b}$ \\
\hline & Total isoflavone & $20.99(20.16-21.83)$ & & $\mathrm{b}$ \\
\hline \multirow{4}{*}{$\begin{array}{l}\text { ROSS, ISOMIL, with iron, powder, } \\
\text { reconstituted as fed }\end{array}$} & Daidzein & 0.78 & 28.8 & None given \\
\hline & Genistein & 1.58 & 58.3 & None given \\
\hline & Glycitein & 0.35 & 12.9 & None given \\
\hline & Total isoflavone & 2.71 & & None given \\
\hline \multirow{3}{*}{$\begin{array}{l}\text { ROSS, ISOMIL, with iron, ready-to- } \\
\text { feed }\end{array}$} & Daidzein & 1.91 & 45.8 & $c$ \\
\hline & Genistein & 2.26 & 54.2 & $\mathrm{c}$ \\
\hline & Total isoflavone & 4.17 & & $\mathrm{c}$ \\
\hline \multirow{4}{*}{$\begin{array}{l}\text { WYETH-AYERST, NURSOY, with } \\
\text { iron, liquid concentrate, not } \\
\text { reconstituted }\end{array}$} & Daidzein & $1.02(0.79-1.25)$ & 25.4 & $\mathrm{~b}$ \\
\hline & Genistein & $2.82(2.19-3.45)$ & 70.1 & $\mathrm{~b}$ \\
\hline & Glycitein & 0.35 & 8.70 & $\mathrm{c}$ \\
\hline & Total isoflavone & $4.02(2.98-5.05)$ & & $\mathrm{b}$ \\
\hline \multirow{4}{*}{$\begin{array}{l}\text { WYETH-AYERST, NURSOY, with } \\
\text { iron, powder, not reconstituted }\end{array}$} & Daidzein & 5.70 & 21.9 & $\mathrm{c}$ \\
\hline & Genistein & 13.55 & 52.1 & $\mathrm{c}$ \\
\hline & Glycitein & 2.05 & 7.9 & $\mathrm{c}$ \\
\hline & Total isoflavone & $26.00(21.30-30.70)$ & & $\mathrm{b}$ \\
\hline \multirow{4}{*}{$\begin{array}{l}\text { WYETH-AYERST, NURSOY, with } \\
\text { iron, ready-to-feed }\end{array}$} & Daidzein & 0.75 & 28.5 & $\mathrm{c}$ \\
\hline & Genistein & 1.60 & 60.8 & $\mathrm{c}$ \\
\hline & Glycitein & 0.28 & 10.6 & $\mathrm{c}$ \\
\hline & Total isoflavone & 2.63 & & $\mathrm{c}$ \\
\hline \multicolumn{5}{|c|}{${ }^{a}$ Brand names are registered to their owners. From USDA (2002b). } \\
\hline \multicolumn{5}{|l|}{${ }^{b}$ Calculated from mean values by CERHR. } \\
\hline
\end{tabular}


Table 4

Estimated Isoflavone+Glycoside Intake by Infants Consuming U.S. Soy Formulas ${ }^{a}$

\begin{tabular}{lccc}
\hline Infant age & Formula intake, mL/day & $\begin{array}{c}\text { Isoflavone+glycoside } \\
\text { intake, mg aglycone } \\
\text { equivalent/day }\end{array}$ & $\begin{array}{c}\text { Body weight, kg } \\
\text { Dose mg/kg bw/ } \\
\text { day }\end{array}$ \\
\hline 1 week & $500-550$ & $22.5-24.8$ & $2.5-3.8$ \\
1 month & $700-800$ & $31.5-36.0$ & $2.9-3.8$ \\
2 months & $800-830$ & $36.0-37.0$ & $3.6-5.9$ \\
4 months & $800-1000$ & $41.0-45.0$ & $4.8-7.5$ \\
\hline
\end{tabular}

${ }^{a}$ From Setchell et al. (1998).

${ }^{b}$ Based on a soy formula concentration of $45 \mu \mathrm{g} / \mathrm{L}$. [The unit of $\mu \mathrm{g}$ seems to be an error; units are listed as mg/L earlier in the report.] 


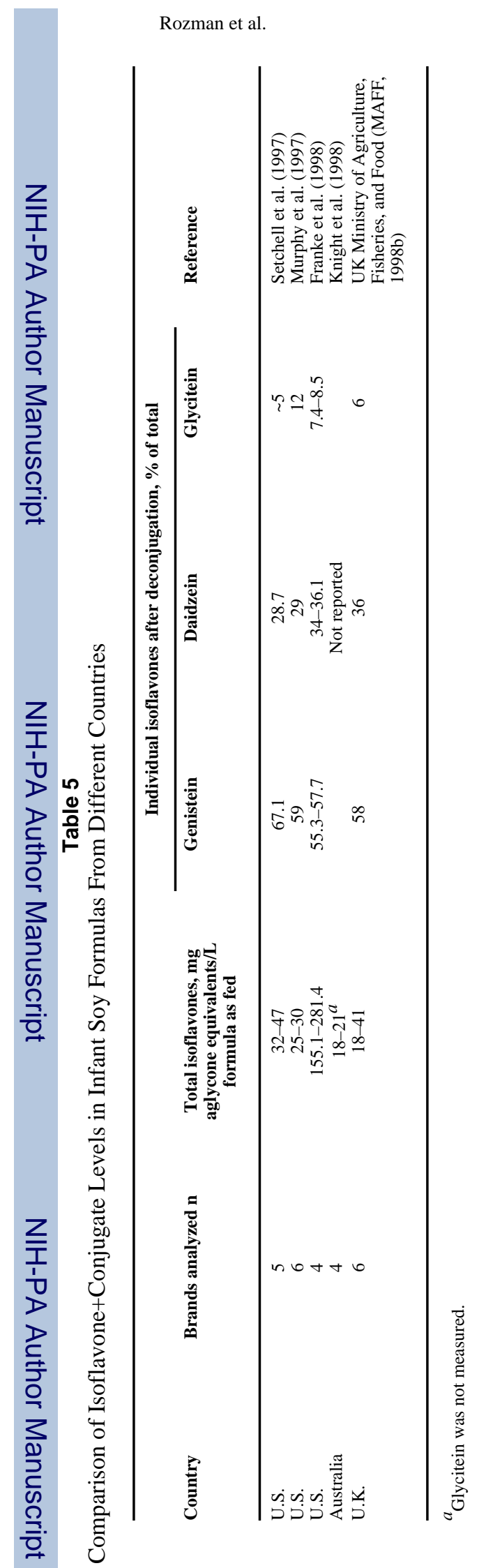

Page 165 
Table 6

Estimated Intake of Isoflavones+Conjugates in Infants Fed Soy Formula

\begin{tabular}{|c|c|c|c|c|}
\hline \multirow[b]{2}{*}{$\begin{array}{l}\text { Country, infant age } \\
\text { (reference) }\end{array}$} & \multicolumn{4}{|c|}{ Intake, mg aglycone equivalents/kg bw/day, based on formula ingestion } \\
\hline & Total Isoflavone & Genistein & Daidzein & Glycitein \\
\hline $\begin{array}{l}\text { U.S., } 4 \text { months (Setchell et al., } \\
\text { 1997) }\end{array}$ & $4.5-8.0 ;\left(6-12^{a}\right)$ & $3.0-5.4(4.0-8.0)$ & $1.3-2.3(1.7-3.4)$ & $0.23-0.4(0.3-0.6)$ \\
\hline $\begin{array}{l}\text { U.S., age not stated (Murphy et } \\
\text { al., 1997) }\end{array}$ & $5-12$ & $3.0-7.1$ & $1.5-3.5$ & $0.60-1.4$ \\
\hline $\begin{array}{l}\text { New Zealand, < } 1 \text { month to } 4 \\
\text { months, (Irvine et al., 1998a,b) }\end{array}$ & $2.9-3.8$ & $1.9-2.4^{b}$ & $1.0-1.4^{b}$ & Not known ${ }^{b}$ \\
\hline $\begin{array}{l}\text { U.K., 1-6 months (MAFF, } \\
\text { 1998b) }\end{array}$ & $4.5-5.0$ & $2.6-2.9$ & $1.6-1.8$ & $0.27-0.30$ \\
\hline $\begin{array}{l}\text { U.S., } 4.5 \text { kg (Franke et al., } \\
\text { 1998) }\end{array}$ & $\sim 1.6$ & $\sim 0.9$ & $\sim 0.5$ & $\sim 0.1$ \\
\hline $\begin{array}{l}\text { U.K., } 4-6 \text { months old (Hoey et } \\
\text { al., 2004) }\end{array}$ & $1.7-4.4$ & $0.99-2.9$ & $0.46-1.3$ & $0.10-0.70$ \\
\hline
\end{tabular}

${ }^{a}$ Values reported in a more recent publication by Setchell et al. (1998).

${ }^{b}$ Percentages of isoflavones are based upon levels of genistein and daidzein reported. It is not known if the formulas also contained glycitein. 
Table 7

Blood Levels of Total Isoflavones by Population

\begin{tabular}{|c|c|c|c|c|}
\hline \multirow[b]{2}{*}{ Population and exposure condition } & \multicolumn{3}{|c|}{ Plasma or serum aglycone equivalents levels, $\mathrm{nM}[\mu \mathrm{g} / \mathrm{L}]($ mean $\pm \mathrm{SD})$} & \multirow[b]{2}{*}{ Reference } \\
\hline & Genistein & Daidzein & Equol & \\
\hline $\begin{array}{l}\text { Seven 4-month old infants fed soy } \\
\text { formula }\end{array}$ & $\begin{array}{l}2530 \pm 1640[684 \\
\pm 443]\end{array}$ & $\begin{array}{l}1160 \pm 230[295 \\
\pm \mathbf{5 8}]\end{array}$ & Not detected & Setchell et al. $(1997,1998)$ \\
\hline Infants fed cow-milk formulas & $11.6 \pm 2.5[3.1 \pm \mathbf{0 . 6 8}]$ & $\begin{array}{l}8.1 \pm 1.1[2.1 \\
\pm \mathbf{0 . 2 8}]\end{array}$ & $\begin{array}{l}16.9 \pm 2.0[4.1 \\
\pm \mathbf{0 . 4 8}]\end{array}$ & Setchell et al. $(1997,1998)$ \\
\hline Infants fed human milk & $10.2 \pm 2.7[\mathbf{2 . 8} \pm \mathbf{0 . 7 6}]$ & $\begin{array}{l}5.86 \pm 0.51[\mathbf{1 . 5} \\
\pm \mathbf{0 . 1 3}]\end{array}$ & Not reported & $\begin{array}{l}\text { Setchell et al. (1997) as cited } \\
\text { in Chen and Rogan (2004) }\end{array}$ \\
\hline $\begin{array}{l}\text { Men consuming traditional Japanese } \\
\text { diet }\end{array}$ & $90-1204$ [24-325] & $\begin{array}{l}\text { 60-924 [15- } \\
\mathbf{2 3 5}]\end{array}$ & $\begin{array}{l}0.54-24.6[\mathbf{0 . 1 3} \\
\pm \mathbf{6 . 0}]\end{array}$ & $\begin{array}{l}\text { Adlercreutz et al. (1994) as } \\
\text { cited in Kurzer and Xu } \\
\text { (1997) }\end{array}$ \\
\hline Omnivorous Japanese men & $276.0[75]$ & $107.0[27]$ & $5.5[1.3]$ & Adlercreutz et al. (1993b) \\
\hline Omnivorous Japanese men & $206.1[56]$ & $72.5[18]$ & Not reported & $\begin{array}{l}\text { Arai et al. (2000) as cited in } \\
\text { Whitten and Patisaul (2001) }\end{array}$ \\
\hline Japanese men & $\begin{array}{l}493.3 \pm 604.4[\mathbf{1 3 3} \\
\pm \mathbf{1 6 3}]\end{array}$ & $\begin{array}{l}280.7 \pm 375.5[71 \\
\pm \mathbf{9 5}]\end{array}$ & Not reported & Pumford et al. (2002) \\
\hline Japanese women & $\begin{array}{l}501.9 \pm 717.6[\mathbf{1 3 6} \\
\pm \mathbf{1 9 4}]\end{array}$ & $\begin{array}{l}246.6 \pm 369.4[63 \\
\pm \mathbf{9 4}]\end{array}$ & Not reported & Pumford et al. (2002) \\
\hline Japanese women & $\begin{array}{l}307.5 \pm 325.4[\mathbf{8 3} \\
\pm \mathbf{8 8}]\end{array}$ & $\begin{array}{l}111.7 \pm 187.8[28 \\
\pm 48]\end{array}$ & Not reported & Arai et al. (2000) \\
\hline Omnivorous Hong Kong men & Not reported & $31.3[\mathbf{8 . 0}]$ & $3.8[\mathbf{0 . 9 2}]$ & $\begin{array}{l}\text { Morton et al. (1997) as cited } \\
\text { in Whitten and Patisaul } \\
\text { (2001) }\end{array}$ \\
\hline Vegetarian Finnish women & $44.8[12]$ & $50[13]$ & $1.5[0.36]$ & Adlercreutz et al. (1993a) \\
\hline Vegetarian Finnish women & $17.1[4.6]$ & $18.5[4.7]$ & $0.7[\mathbf{0 . 1 7}]$ & $\begin{array}{l}\text { Adlercreutz et al. (1994) as } \\
\text { cited in Whitten and Patisaul } \\
\text { (2001) }\end{array}$ \\
\hline Lactovegetarian Finnish women & $29.7[8.0]$ & $41.5[11]$ & $1.0[\mathbf{0 . 0 5 9}]$ & $\begin{array}{l}\text { Adlercreutz et al. (1994) as } \\
\text { cited in Whitten and Patisaul } \\
\text { (2001) }\end{array}$ \\
\hline Omnivorous Finnish women & $7.7[2.0]$ & 6.4 [1.6] & $1.6[0.39]$ & Adlercreutz et al. (1993a) \\
\hline Omnivorous Finnish women & 4.9 [1.3] & 4.2 [1.1] & $0.8[\mathbf{0 . 1 9}]$ & $\begin{array}{l}\text { Adlercreutz et al. (1994) as } \\
\text { cited in Kurzer and Xu } \\
\text { (1997) and Whitten and } \\
\text { Patisaul (2001) }\end{array}$ \\
\hline Finnish men & $6.3[1.7]$ & $6.2[1.6]$ & 0.8 [0.19] & Adlercreutz et al. (1993a) \\
\hline Omnivorous Finnish men & $0.5[\mathbf{0 . 1 4}]$ & $0.6[\mathbf{0 . 1 5}]$ & $0.1[\mathbf{0 . 0 2 4}]$ & $\begin{array}{l}\text { Adlercreutz et al. (1993) as } \\
\text { cited in Whitten and Patisaul } \\
\text { (2001) }\end{array}$ \\
\hline Omnivorous Spanish men & Not reported & $1.3[\mathbf{0 . 3 3}]$ & $0.4[\mathbf{0 . 1 0}]$ & $\begin{array}{l}\text { Morton et al. (1997) as cited } \\
\text { in Whitten and Patisaul } \\
\text { (2001) }\end{array}$ \\
\hline Omnivorous Canadian men & $8.2[2.2]$ & $3.4[\mathbf{0 . 8 6}]$ & Not reported & $\begin{array}{l}\text { Gooderham et al. (2000) as } \\
\text { cited in Whitten and Patisaul } \\
(2001)\end{array}$ \\
\hline Omnivorous British men & Not reported & $8.2[\mathbf{2 . 1}]$ & $0.6[\mathbf{0 . 1 5}]$ & $\begin{array}{l}\text { Morton et al. (1997) as cited } \\
\text { in Whitten and Patisaul } \\
\text { (2001) }\end{array}$ \\
\hline British men & $34.1 \pm 27.2[\mathbf{9 . 2} \pm 7.4]$ & $\begin{array}{l}18.2 \pm 20.4[4.6 \\
\pm 5.2]\end{array}$ & Not reported & Pumford et al. (2002) \\
\hline British women & $30.1 \pm 31.2[\mathbf{8 . 1} \pm \mathbf{8 . 4}]$ & $\begin{array}{l}13.5 \pm 11.6[3.4 \\
\pm \mathbf{2 . 9}]\end{array}$ & Not reported & Pumford et al. (2002) \\
\hline
\end{tabular}


Table 8

Blood Levels of Isoflavones After Ingestion of Soy Foods or Supplements ${ }^{a}$

Plasma or serum levels, nM [ $\mu \mathrm{g} / \mathrm{L}]$, aglycone equivalents, mean \pm SD

Population and exposure condition

\section{Genistein}

Daidzein

Reference

Women ingesting $0.7 \mathrm{mg} / \mathrm{kg}$ bw isoflavones $(44 \%$ genistein and $56 \%$ daidzein) through soy milk

powder

Women ingesting $1.3 \mathrm{mg} / \mathrm{kg}$ bw isoflavones $(44 \%$

genistein and $56 \%$ daidzein) through soy milk

powder

Women ingesting $2.0 \mathrm{mg} / \mathrm{kg}$ bw isoflavones (44\%

genistein and $56 \%$ daidzein) through soy milk

powder

Women consuming $4.5 \mu \mathrm{mol} / \mathrm{kg}$ bw isoflavones

through soy milk (48.9\% genistein, $43.3 \%$ daidzein,

$7.8 \%$ glycitein)

Women consuming $4.5 \mu \mathrm{mol} / \mathrm{kg}$ bw isoflavones

through soy germ (12.6\% genistein, $48.5 \%$

daidzein, $38.9 \%$ glycitein $)^{b}$

Men consuming $4.5 \mu \mathrm{mol} / \mathrm{kg}$ bw isoflavones

through soy milk (48.9\% genistein, $43.3 \%$ daidzein,

$7.8 \%$ glycitein $)^{b}$

Men consuming $4.5 \mu \mathrm{mol} / \mathrm{kg}$ bw isoflavones

through soy germ (12.6\% genistein, $48.5 \%$

daidzein, $38.9 \%$ glycitein $)^{b}$

Males ingesting cereal bar containing $8 \mathrm{~g}$ defatted

soy grit ( 20mg isoflavones)

Males ingesting cake containing $10.95 \mathrm{mg}$ genistein

and $8.54 \mathrm{mg}$ daidzein for 3 days; Day 3 values listed

Males ingesting isoflavones $16 \mathrm{mg} / \mathrm{kg}$ bw

Males consuming soy protein-isolate beverage (60g/day) for 28 days

Male ingesting soy supplement at dose of $35.6 \mathrm{mg} /$ day daidzein and $5.6 \mathrm{mg} /$ day genistein for 7 days

Female ingesting soy supplement at dose of

$35.6 \mathrm{mg} /$ day daidzein and $5.6 \mathrm{mg} /$ day genistein for

7 days

Females ingesting $5 \mathrm{mg}$ genistein or $5 \mathrm{mg}$ daidzein

$740 \pm 440[200 \pm 119]$

$1070 \pm 630[\mathbf{2 8 9} \pm \mathbf{1 7 0}]$

$2150 \pm 1330[\mathbf{5 8 1} \pm \mathbf{3 5 9}]$

$1700 \pm 1010[\mathbf{4 5 9} \pm \mathbf{2 7 3}]$

$510 \pm 190[\mathbf{1 3 8} \pm \mathbf{5 1}]$

$1780 \pm 830[481 \pm 224]$

$470 \pm 290[127 \pm 78]$

$790 \pm 40[\mathbf{2 0 1} \pm \mathbf{1 0}]$

$1220 \pm 670[\mathbf{3 1 0} \pm \mathbf{1 7 0}]$

$2240 \pm 1180[\mathbf{5 7 0} \pm \mathbf{3 0 0}]$

$1040 \pm 610[\mathbf{2 6 4} \pm \mathbf{1 5 5}]$

$1630 \pm 1030[\mathbf{4 1 4} \pm \mathbf{2 6 2}]$

$1290 \pm 500[\mathbf{3 2 8} \pm \mathbf{8 3}]$

$1160 \pm 440[\mathbf{2 9 5} \pm \mathbf{1 1}]$

Xu et al. (1994)

Females ingesting $5 \mathrm{mg}$ genistein or $5 \mathrm{mg}$ daidzein
468 [126]

445 [120]

$7700[\mathbf{2 0 8 1}]$ (total) 70

(free) [19]

$907 \pm 245[\mathbf{2 4 5} \pm \mathbf{6 6}]$

$138 \pm 13[37.3 \pm 3.5]$

$383 \pm 16[\mathbf{1 0 4} \pm \mathbf{4 . 3}]$

$1220 \pm 470^{c}[\mathbf{3 3 0} \pm \mathbf{1 2 7}]$

$1260 \pm 270^{c}[341 \pm 73]$
392 [100]

297 [75.5]

Not reported

498 \pm 102 nM [127 \pm 26$]$

$671 \pm 46[\mathbf{1 7 1} \pm \mathbf{1 2}]$

$558 \pm 14[142 \pm 3.6]$

$1550 \pm 240^{c}[394 \pm 61]$

$760 \pm 120^{c}[\mathbf{1 9 3} \pm 49]$
Xu et al. (1994)

Xu et al. (1994)

Zhang et al. (1999)

Zhang et al. (1999)

Zhang et al. (1999)

Zhang et al. (1999)

Pumford et al. (2002)

Pumford et al. (2002)

Busby et al. (2002) as cited in UK Committee on Toxicity (2003)

Gooderham et al. (1996)

as cited in ILSI (1999)

Doerge et al. (2000)

Doerge et al. (2000)

Setchell et al. (2001) Setchell et al. (2001)

${ }^{a}$ Equol was not reported in these studies.

$b_{\text {Plas }}$

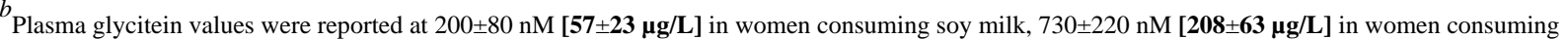

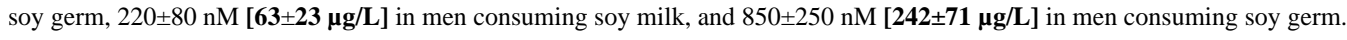

${ }^{c}$ Variance not specified. 
Rozman et al.

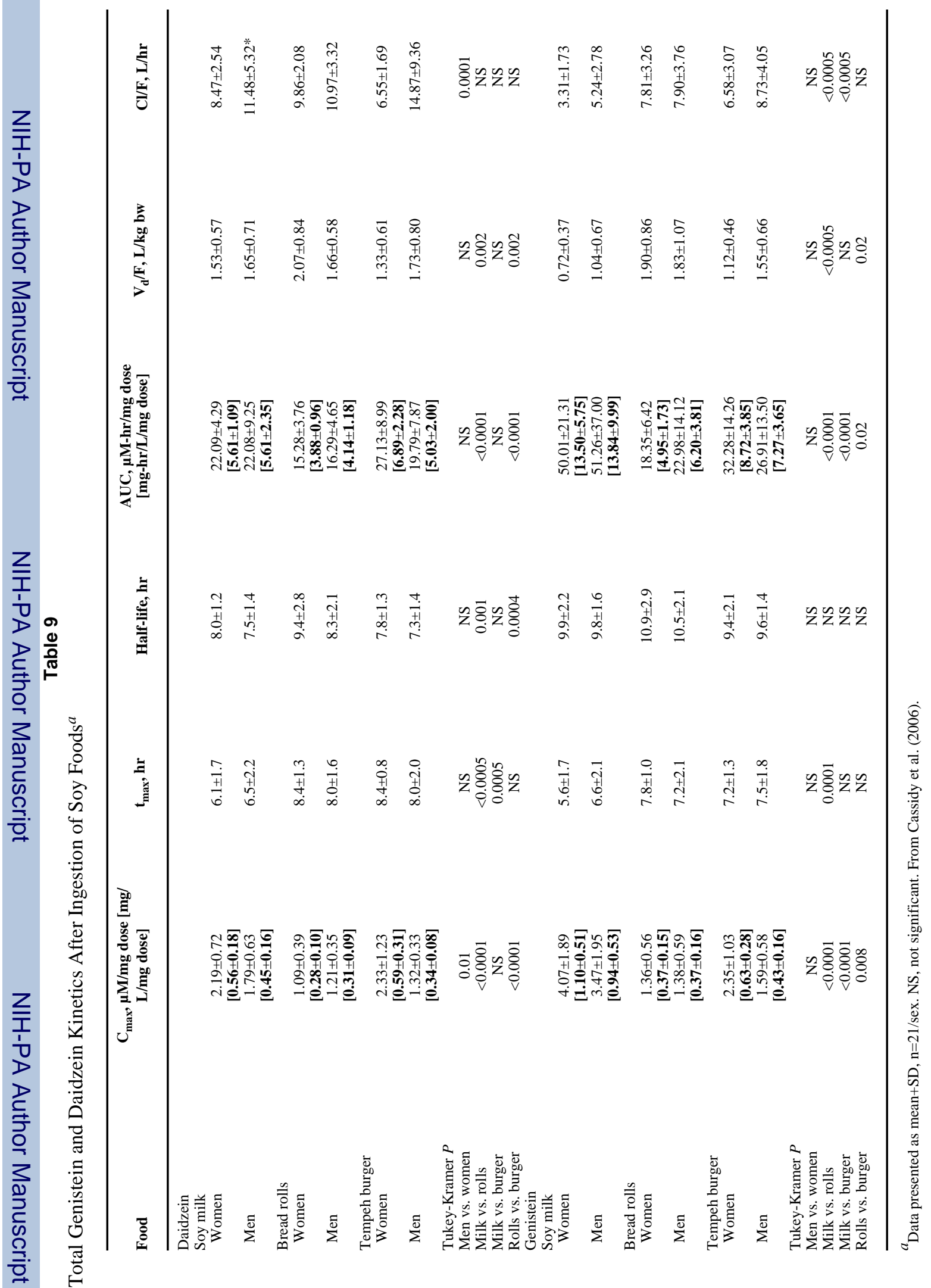

Birth Defects Res B Dev Reprod Toxicol. Author manuscript; available in PMC 2008 March 11. 


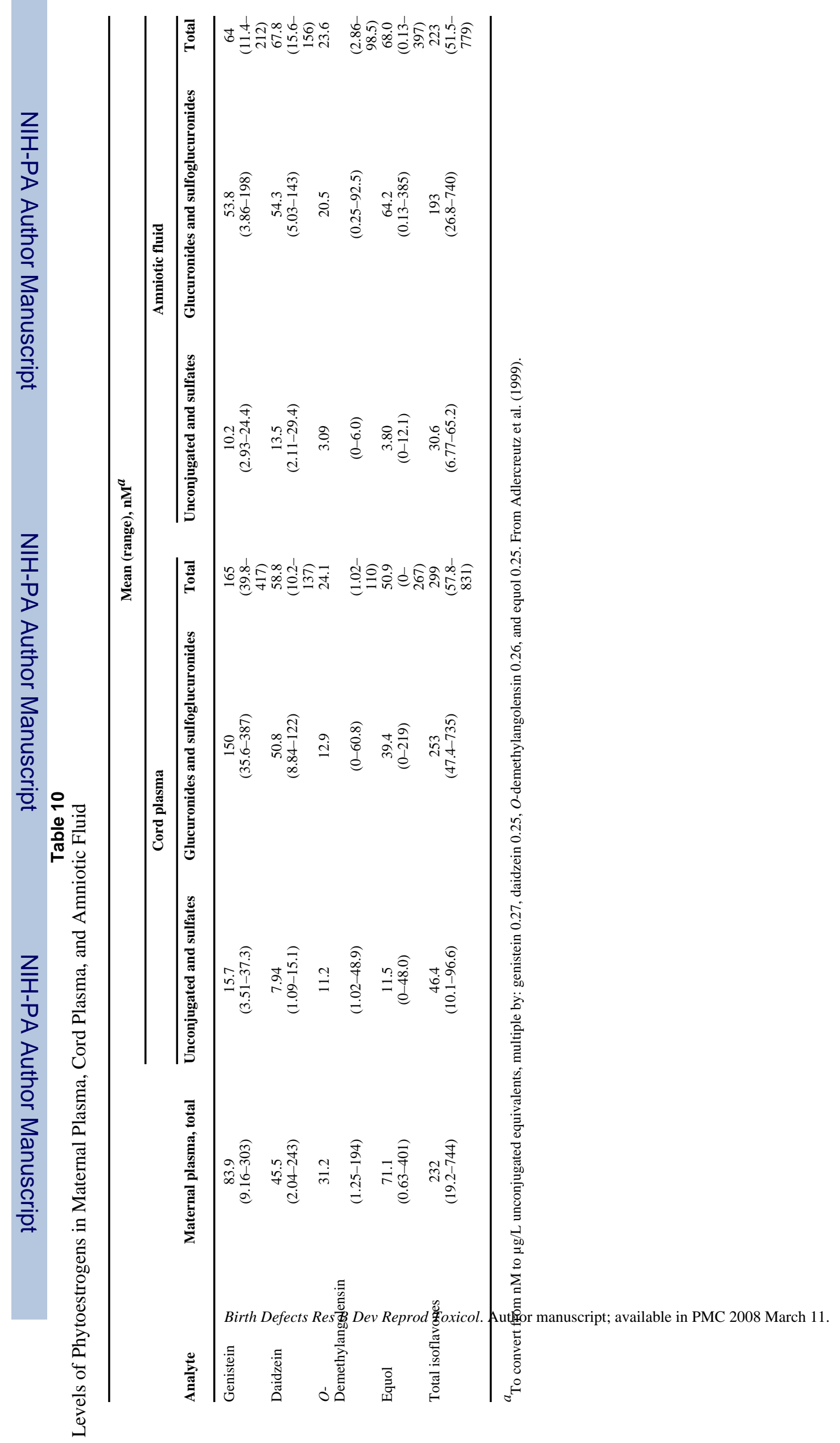


Table 11

Correlations Between Isoflavone Levels in Maternal Plasma, Cord Blood, and Amniotic Fluid

\begin{tabular}{llc}
\hline & \multicolumn{2}{c}{ Pearson Correlation } \\
\cline { 2 - 3 } Isoflavone & Maternal plasma vs. cord plasma & Maternal plasma vs. amniotic fluid \\
\hline Genistein & 0.338 & 0.530 \\
Daidzein & $0.439^{*}$ & $0.418 *$ \\
$O$-Demethylangolensin & $0.967^{*}$ & $0.970^{*}$ \\
Equol & $0.997^{*}$ & $0.997^{*}$ \\
\hline
\end{tabular}

${ }^{*} P<0.001$. From Adlercreutz et al. (1999). 
Table 12

Mean Recoveries of Genistein and Daidzein in Humans

\begin{tabular}{|c|c|c|c|c|}
\hline \multirow[b]{2}{*}{ Population and exposure condition ${ }^{a}$} & \multicolumn{3}{|c|}{ Percent intake excreted within $24-48 \mathrm{hr}^{b}$} & \multirow[b]{2}{*}{ Reference } \\
\hline & Genistein & Daidzein & Total isoflavones & \\
\hline $\begin{array}{l}\text { Four infants fed soy formulas containing } 81- \\
92 \mathrm{mg} / \mathrm{kg} \text { genistein and } 44-55 \mathrm{mg} / \mathrm{kg} \text { daidzein }\end{array}$ & $13 \pm 3(\mathrm{SEM})$ & $38 \pm 4$ & Not reported & Irvine et al. (1998b) \\
\hline $\begin{array}{l}\text { Women consuming soy milk powder }(56.0 \% \\
\text { genistein and } 44.0 \% \text { daidzein) with } 0.9 \mathrm{mg} / \mathrm{kg} \\
\text { bw isoflavone } / \mathrm{meal} 3 \text { times daily with various } \\
\text { background diets }\end{array}$ & $18-20$ & $26-27$ & $25-27$ & $\mathrm{Xu}$ et al. (2000) \\
\hline $\begin{array}{l}\text { Women consuming soybeans, texturized } \\
\text { vegetable protein, tofu, or tempeh }(54-60 \% \\
\text { genistein and } 40-46 \% \text { daidzein) }\end{array}$ & $9-16$ & $38-51$ & $21-34$ & Xu et al. (2000) \\
\hline $\begin{array}{l}\text { Women consuming } 0.7,1.3 \text {, and } 2.0 \mathrm{mg} \\
\text { isoflavones through soy milk ( } 44 \% \text { genistein } \\
\text { and } 56 \% \text { daidzein) }\end{array}$ & $5-11$ & $19-23$ & $15-19$ & Xu et al. (1994) \\
\hline $\begin{array}{l}\text { Women drinking soy milk providing doses of } \\
\sim 85 \mathrm{mg} / \text { day genistein and } 69 \mathrm{mg} / \text { day daidzein }\end{array}$ & 11 & 35 & 22 & Lu et al. (2000a) \\
\hline $\begin{array}{l}\text { Women drinking soy milk containing } 80-200 \\
\text { mg of each isoflavone }\end{array}$ & 24 & 66 & Not reported & $\begin{array}{l}\text { Lu and Anderson } \\
\text { (1998) }\end{array}$ \\
\hline $\begin{array}{l}\text { Men drinking soy milk containing } 80-200 \mathrm{mg} \\
\text { of each isoflavone }\end{array}$ & 15 & 47 & Not reported & $\begin{array}{l}\text { Lu and Anderson } \\
\text { (1998) }\end{array}$ \\
\hline $\begin{array}{l}\text { Men drinking soy milk containing } ~ 100 \mathrm{mg} \\
\text { each of genistein and daidzein }\end{array}$ & 15 & 47 & Not reported & Lu et al. (1995) \\
\hline Men consuming baked soybean powder & 18 & 36 & Not reported & $\begin{array}{l}\text { Watanabe et al. as } \\
\text { cited by UK } \\
\text { Committee on } \\
\text { Toxicity (2003) }\end{array}$ \\
\hline $\begin{array}{l}\text { Women and men consuming } 4.5 \mu \mathrm{mol} \\
\text { isoflavones through soy milk }(48.9 \% \\
\text { genistein, } 43.3 \% \text { daidzein, } 7.8 \% \text { glycitein })^{c}\end{array}$ & 27.6 & 48.6 & Not reported & Zhang et al. (1999) \\
\hline $\begin{array}{l}\text { Women and men consuming } 4.5 \mu \mathrm{mol} \\
\text { isoflavones through soy germ }(12.6 \% \\
\text { genistein, } 48.5 \% \text { daidzein, } 38.9 \% \text { glycitein })\end{array}$ & 29.7 & 43.8 & Not reported & Zhang et al. (1999) \\
\hline $\begin{array}{l}\text { Women consuming soy protein } 60 \mathrm{~g} / \text { day } \\
(25.08 \mathrm{mg} / \text { day daidzein and } 19.85 \mathrm{mg} / \mathrm{day} \\
\text { genistein) during } 1 \text { menstrual cycle }\end{array}$ & {$[0.4-12]$} & {$[0.8-16]$} & $1.8-12.9$ & Cassidy et al. (1994) \\
\hline $\begin{array}{l}\text { Men and women consuming } 1-16 \mathrm{mg} / \mathrm{kg} \text { bw } \\
\text { formulations containing } 70-100 \% \\
\text { unconjugated isoflavones }\end{array}$ & $8-18$ & $26-53$ & $34-74$ & $\begin{array}{l}\text { Bloedon et al. (2002); } \\
\text { Busby et al. (2002) }\end{array}$ \\
\hline
\end{tabular}

Birth Defects Res B Dev Reprod Toxicol. Author manuscript; available in PMC 2008 March 11. 
Table 13

Isoflavone Levels in Plasma of Rat Dams and Offspring ${ }^{a}$

\begin{tabular}{|c|c|c|c|}
\hline \multirow[b]{2}{*}{ Rat } & \multicolumn{3}{|c|}{ Plasma aglycone equivalent levels $(\mu \mathrm{g} / \mathrm{L})[\mathrm{nM}]$} \\
\hline & Genistein & Daidzein & Equol \\
\hline Fetus, GD 20.5 & $106.39[394]$ & $67.53[266]$ & $51.58[213]$ \\
\hline DaM, GD 20.5 & 232.31 [860] & 193.80 [762] & 578.12 [2387] \\
\hline Pup, PND 3.5 & 234.85 [869] & 341.13 [1342] & $161.85[668]$ \\
\hline Dam, PND 3.5 & $441.22[\mathbf{1 6 3 3}]$ & $339.46[\mathbf{1 3 3 5}]$ & $906.22[3742]$ \\
\hline Adult male & $420.95[\mathbf{1 5 5 8}]$ & $390.27[\mathbf{1 5 3 5}]$ & $932.37[\mathbf{3 8 5 0}]$ \\
\hline
\end{tabular}

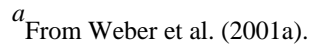

GD, gestational day; PND, postnatal day. 
Table 14

Isoflavone Serum Isoflavone Levels in Rodents of Offspring Fed a Soy-Based Diet ${ }^{a}$

\begin{tabular}{llcc}
\hline & & Aglycone equivalents, $\mathbf{n g} / \mathbf{m L} \mathbf{b}_{[\mathbf{n M}]}$ \\
\cline { 2 - 4 } Pup age & Genistein & Daidzein & Equol \\
\hline Newborn $c$ & $100[\mathbf{3 7 0}]$ & $50[\mathbf{1 9 7}]$ & $550[\mathbf{2 2 7 1 ]}$ \\
6-day-old & $90[\mathbf{3 3 3}]$ & $50[\mathbf{1 9 7 ]}$ & $80[\mathbf{3 3 0}]$ \\
12-day-old & $110[\mathbf{4 0 7}]$ & $25[\mathbf{9 8}]$ & $70[\mathbf{2 8 9}]$ \\
16-day-old & $10[\mathbf{3 7}]$ & $5[\mathbf{2 0}]$ & $60[\mathbf{2 4 8}]$ \\
\hline
\end{tabular}

${ }^{a}$ From Brown and Setchell (2001).

${ }^{b}$ Values estimated from a graph by CERHR.

${ }^{c}$ Measured in pups before nursing. 
Table 15

Phytoestrogen Levels in Brains of Male Rats Fed a Phytoestrogen-Containing Diet $^{a}$

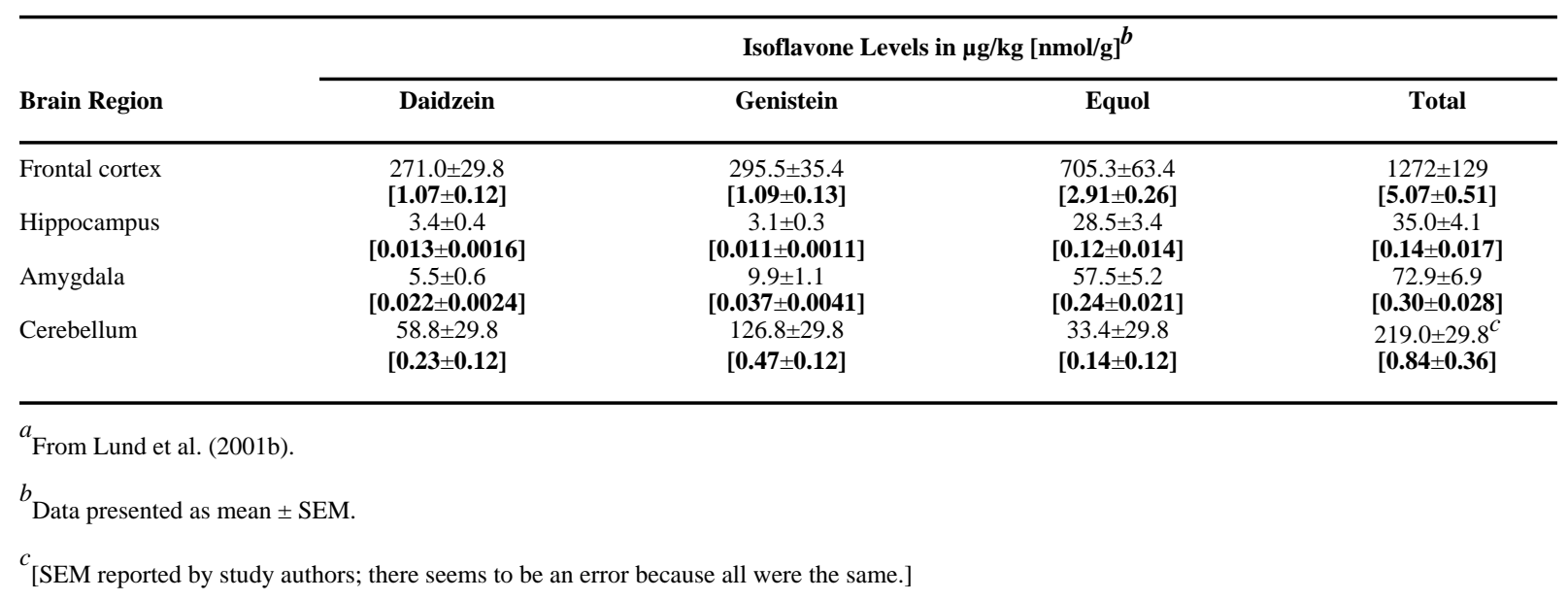




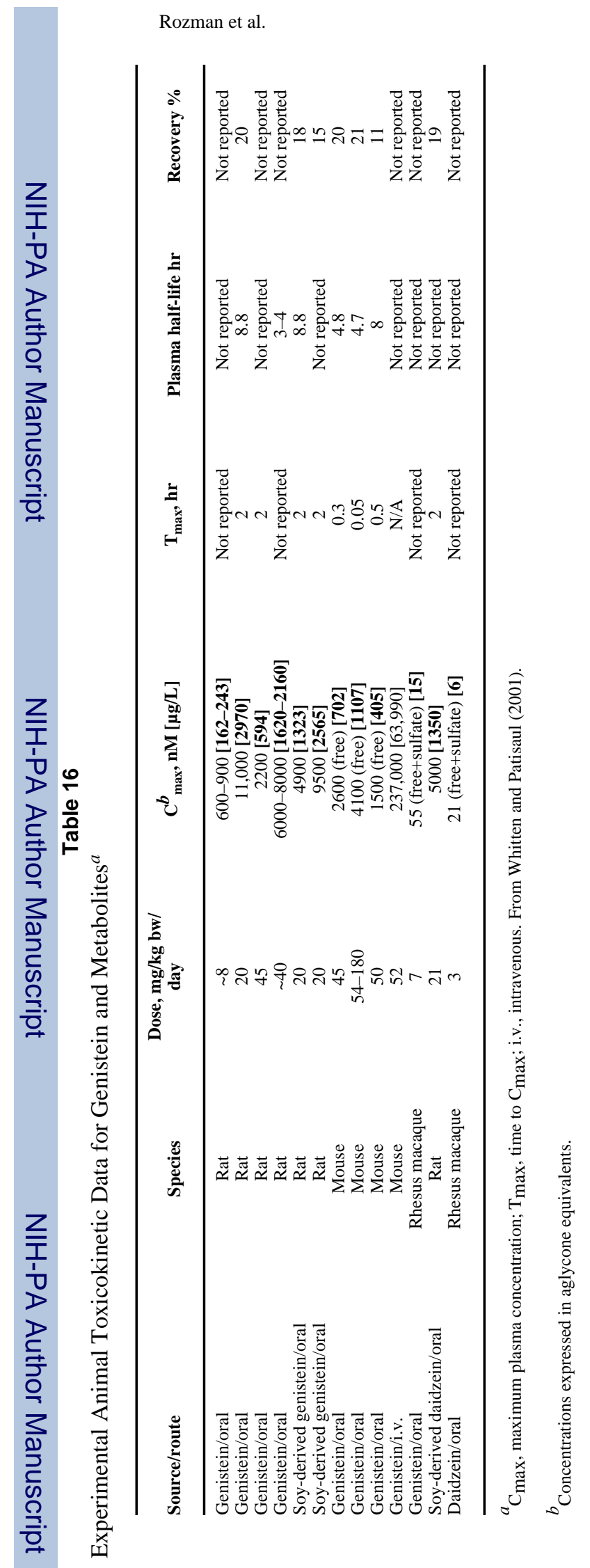

Page 176 
Table 17

Summary of Studies Examining Estrogenicity Endpoints in Humans ${ }^{a}$

\begin{tabular}{|c|c|c|c|}
\hline Study Description $b$ & Endpoint & Effect & Reference \\
\hline 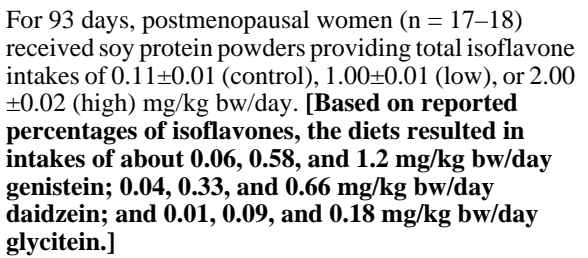 & $\begin{array}{l}\text { Vaginal cytology and } \\
\text { endometrial biopsy } \\
\text { Plasma levels of } 17 \beta- \\
\text { estradiol, estrone, } \\
\text { dehydroepiandrosterone- } \\
\text { sulfate, sex hormone-binding } \\
\text { globulin, insulin, thyroid- } \\
\text { binding globulin }\end{array}$ & $\begin{array}{l}\text { No significant change } \\
\text { Slight decreases compared to } \\
\text { baseline values, but the study } \\
\text { authors stated effects were } \\
\text { modest and unlikely to be of } \\
\text { physiologic significance }\end{array}$ & $\begin{array}{l}\text { Duncan et al. } \\
(1999)\end{array}$ \\
\hline $\begin{array}{l}\text { Postmenopausal women received a soy diet }(\mathrm{n}=66) \\
\text { with soy products representing one-third of caloric } \\
\text { intake or their usual diet (control group, } \mathrm{n}=25) \text { for } 4 \\
\text { weeks. Foods were analyzed by HPLC, and it was } \\
\text { determined that the soy diets provided } 165 \mathrm{mg} / \mathrm{day} \\
\text { isoflavones [40.3 } \mathbf{~ m g / d a y ~ g e n i s t e i n ~ a n d ~} \mathbf{1 2 4 . 8} \mathbf{~ m g / d a y} \\
\text { daidzein. Based on } \mathbf{5 8} \mathbf{~ k g ~ b w : ~} 0.69 \mathrm{mg} / \mathbf{k g ~ b w} / \mathbf{d a y} \\
\text { genistein and } 2.2 \mathrm{mg} / \mathbf{k g ~ b w / d a y ~ d a i d z e i n . ] ~}\end{array}$ & $\begin{array}{l}\text { Vaginal superficial cells } \\
\text { Serum FSH, LH, 17ß- } \\
\text { estradiol, or sex hormone- } \\
\text { binding globulin levels }\end{array}$ & $\begin{array}{l}\text { No significant change } \\
\text { No significant change }\end{array}$ & $\begin{array}{l}\text { Baird et al. } \\
\text { (1995) }\end{array}$ \\
\hline $\begin{array}{l}\text { Postmenopausal women were randomized to receive } 46 \\
\text { g/day of either soy }(n=23) \text { or wheat }(n=24) \text { flour for } \\
12 \text { weeks. }\end{array}$ & Vaginal maturation index & No significant change & $\begin{array}{l}\text { Murkies et al. } \\
\text { (1995) }\end{array}$ \\
\hline $\begin{array}{l}\text { In a study with Latin-square design, postmenopausal } \\
\text { women }(\mathrm{n}=23) \text { were given } 45 \mathrm{~g} \text { soy flour, } 10 \mathrm{~g} \text { red } \\
\text { clover sprouts, or } 25 \mathrm{~g} \text { linseed daily, during separate } 2 \text { - } \\
\text { week periods. Vaginal smears were taken after each } \\
\text { supplementation period, and cumulative effects of the } \\
3 \text { foods were compared at } 6 \text { weeks and at } 2 \text { and } 8 \text { weeks } \\
\text { after supplementation. }\end{array}$ & $\begin{array}{l}\text { Vaginal maturation index } \\
\text { FSH/LH levels }\end{array}$ & $\begin{array}{l}\text { Significant changes in vaginal } \\
\text { cytology after } 6 \text { weeks of } \\
\text { supplementation; changes } \\
\text { persisted for } 2 \text { weeks. } \\
\text { Increased after soy } \\
\text { supplementation } \\
\text { Concentrations remained } \\
\text { higher than premenopausal } \\
\text { values. }\end{array}$ & $\begin{array}{l}\text { Wilcox et al. } \\
(1990)\end{array}$ \\
\hline $\begin{array}{l}\text { Healthy menopausal women were randomized to } \\
\text { receive for } 5 \text { years placebo }(\mathrm{n}=197) \text { or } 3 \text { extract tablets/ } \\
\text { day }(\mathrm{n}=179) \text {, each containing } 150 \mathrm{mg} \text { total isoflavones } \\
\text { consisting of genistein } 40-45 \% \text {, daidzein } 40-45 \% \text {, and } \\
\text { glycitein } 10-20 \% \text {. }\end{array}$ & $\begin{array}{l}\text { Endometrial proliferation or } \\
\text { hyperplasia }\end{array}$ & $\begin{array}{l}\text { Baseline: Proliferative } \\
\text { endometria in } 2 \text { women of the } \\
\text { soy extract group and } 3 \\
\text { women of the placebo group; } \\
\text { hyperplasia was an exclusion } \\
\text { criterion. } \\
\text { At } 30 \text { months: No cases of } \\
\text { proliferation or hyperplasia. } \\
\text { At } 5 \text { years: No proliferation or } \\
\text { hyperplasia in placebo group; } \\
5 \text { biopsies ( } 3.2 \%) \text { were } \\
\text { proliferative and } 6 \text { biopsies } \\
(3.8 \%) \text { were hyperplastic in } \\
\text { the soy-extract group. }\end{array}$ & $\begin{array}{l}\text { Unfer et al. } \\
(2004)\end{array}$ \\
\hline $\begin{array}{l}\text { Before scheduled biopsy, women with benign or } \\
\text { malignant breast conditions but normal menstrual } \\
\text { cycles were assigned to a control group }(n=29) \text { or a } \\
\text { soy-supplement group }(n=19) \text {. Women in the soy- } \\
\text { supplement group ate bread rolls containing } 60 \mathrm{~g} \text { soy } \\
\text { supplement as textured vegetable protein }(45 \mathrm{mg} \\
\text { isoflavones) for } 14 \text { days. Normal breast tissue was } \\
\text { collected near lesions. }\end{array}$ & $\begin{array}{l}\text { Proliferation of breast } \\
\text { lobular epithelium and } \\
\text { progesterone receptor } \\
\text { expression. }\end{array}$ & $\begin{array}{l}\text { Both endpoints increased } \\
\text { when controlled for stage of } \\
\text { menstrual cycle and age in the } \\
\text { preliminary study. The final } \\
\text { report, with } 33 \text { tissue bank } \\
\text { controls and some other } \\
\text { subjects added, found no } \\
\text { differences between the } \\
\text { control group and the group } \\
\text { eating soy rolls. }\end{array}$ & $\begin{array}{l}\text { McMichael- } \\
\text { Phillips et al. } \\
\text { (1998); } \\
\text { Hargreaves et } \\
\text { al. (1999) }\end{array}$ \\
\hline
\end{tabular}

\footnotetext{
$a_{\mathrm{FSH}}$, follicle-stimulating hormone; LH, luteinizing hormone.

b

${ }^{b}$ Isoflavone content assumed to be expressed in aglycone equivalents.
} 
Table 18

Uterotropic Responses in Rats Fed Infant Formulas or Diethylstilbestrol ${ }^{a}$

\begin{tabular}{|c|c|c|}
\hline Treatment & $\begin{array}{r}\text { Strength } \% \text { of recommended dilution for } \\
\text { feeding infants }\end{array}$ & Uterine wet weight $\%$ of negative control $b$ \\
\hline \multicolumn{3}{|l|}{ Cow-milk formula } \\
\hline SMA gold & 100 & $132 \%^{c}$ \\
\hline \multicolumn{3}{|l|}{ Soy formula } \\
\hline Wysoy & 100 & $140 \%^{d}$ \\
\hline Farley's & 100 & $125 \% d$ \\
\hline \multirow{3}{*}{ Infasoy } & 33 & $116 \%$ \\
\hline & 100 & $148-179 \%{ }^{d}$ \\
\hline & 200 & $167 \%{ }^{c}$ \\
\hline Diethylstilbestrol, $10 \mu \mathrm{g} / \mathrm{L}$ & & $156-196 \% d$ \\
\hline \multicolumn{3}{|l|}{${ }^{a}$ From Ashby et al. (2000b). } \\
\hline \multicolumn{3}{|c|}{$b_{\text {Estimated from graphs by CERHR. }}$} \\
\hline \multicolumn{3}{|l|}{${ }^{c}{ }_{P<0.05}$} \\
\hline
\end{tabular}


Table 19

Soy Estrogenicity in Laboratory Animals ${ }^{a}$

\begin{tabular}{l} 
Animal \\
\hline $\begin{array}{l}\text { Alpk rat, } 21 \text { days } \\
\text { old }\end{array}$ \\
Sprague-Dawley \\
rat, $>40$ days old,
\end{tabular}

rat, $>40$ days old ovariectomized

Female SpragueDawley rat, 24 days old

Female F344 rat, 3 months old

Male adult NMRI mouse exposed to diethylstilbestrol as neonate and castrated in adulthood.

Female HanNMRI mouse, 16 days old

Female CD- 1 mouse, 15 days old

$$
\begin{aligned}
& \text { Design } \\
& \text { Rats were fed RM1 (standard) } \\
& \text { diet or AIN-76A } \\
& \text { (phytoestrogen-free diet) for } 3 \\
& \text { days. Some rats fed AIN-76A } \\
& \text { diet were also administered the } \\
& \text { anti-estrogen Faslodex. } \\
& \text { Rats were fed a soy diet } \\
& \text { containing } 117.8 \text { mg } \\
& \text { isoflavone/1800 calories or an } \\
& \text { alcohol-extracted soy diet with } \\
& 11.6 \text { mg isoflavone/1800 } \\
& \text { calories for } 2 \text { months. } \\
& \text { Rats were fed casein diets or } \\
& \text { soy diets containing low or } \\
& \text { high isoflavone levels (as } \\
& \text { described above) }+ \text { conjugated } \\
& \text { equine estrogen at } 0.313 \text { or } \\
& 0.625 \text { mg/1800 calories. }
\end{aligned}
$$

Multigeneration design using casein-based diet or alcoholwashed, isoflavone-poor soy protein diet. A commercial soy extract was added to the isoflavone-poor diet, providing isoflavone levels of 31.7$1046.6 \mathrm{mg} / \mathrm{kg}$ feed. Juvenile $F_{2}$ females were evaluated on PND 4. Some females were given s.c. ethinyl estradiol or bisphenol A from PND 21.

For 14 weeks, rats (10-14/ group) were fed either a caseinbased diet, a diet containing $100 \mathrm{~g} / \mathrm{kg}$ isolated soy protein (2.14 $\mathrm{mg}$ aglycones/g isoflavone), a diet containing $200 \mathrm{mg} / \mathrm{kg}$ isolated soy protein a casein-based diet containing $17.2 \mathrm{~g} / \mathrm{kg}$ isoflavones (11.37 $\mathrm{mg}$ aglycones/g isoflavones), or a casein-based diet containing $34.4 \mathrm{~g} / \mathrm{kg}$ isoflavones.

Mice were fed soy-free diets or diets containing $7 \%$ roasted soy meal, for up to 10-20 days after castration; $17 \beta$-estradiol was given to some mice in each dietary group.

Mice weaned at 16 days old to soy-free diet or diet containing $7 \%$ roasted soy meal.

diethylstilbestrol was added to some diets $(6 \mu \mathrm{g} / \mathrm{kg}$ [ $\mathbf{k g}$ feed assumed]).

Mice were weaned at 15 days of age and fed 1 of the following diets for 3,5 , or 7 days: Rodent Chow \#5002 (no information on dietary components); Rodent Chow 5001 (reported to have high isoflavone levels and assumed to be soy based); Mouse Chow \#5015 (reported to have high isoflavone levels and assumed to be soy based); NIH-07 (12\% soybean meal); NIH-31 (5\% soybean meal); or AIN-76A (casein based).

\begin{tabular}{lll} 
Endpoint(s) & Results & Reference \\
\hline Uterine weight. & $\begin{array}{l}\text { Higher in rats fed AIN-76A } \\
\text { diet compared to RM1 diet, } \\
\text { an effect that was eliminated } \\
\text { with Faslodex treatment. The } \\
\text { authors had no explanation } \\
\text { for the results. }\end{array}$ & $\begin{array}{l}\text { Ashby et al. } \\
(2000 \mathrm{a}, \mathrm{b})\end{array}$ \\
$\begin{array}{l}\text { No significant effects. } \\
\text { Vaginal cytology, uterine }\end{array}$ & $\begin{array}{l}\text { Tansey et } \\
\text { al. (1998) }\end{array}$ &
\end{tabular}

weight, endometrial cell

expression, luminal epithelial cell height, or apoptosis.

Vaginal cytology, uterine weight, endothelial cell proliferation, and apoptosis.

Estrogen induced changes in all parameters examined; soy isoflavones did not further affect these parameters.

Lactoferrin staining.

Luminal epithelial cell height.

High isoflavones attenuated staining induced by estrogen. High isoflavones attenuated increase induced by estrogen All three estrogenic

Uterine weight, peroxidase, and epithelial height.

endpoints were increased by the highest isoflavone diet (1046.6 mg/kg feed). There was no interaction with ethinyl estradiol or bisphenol A except additivity between ethinyl estradiol and isoflavones at the highest dietary level.

Uterine wet weight and histopathology.

No significant effects.

Nakai et al. (2005)

Prostatic metaplastic

Soy diets did not affect either Mäkelä et transformation and expression of c-fos oncogene (endpoints of estrogenic action) endpoint and did not alter estrogenic effects in mice exposed to $17 \beta$-estradiol.

Relative uterine weight after 7

Relative uterine weight increased $10-15 \%$ by soy diet. Soy diet decreased the diethylstilbestrol-associated increase in relative uterine weight.

Uterine:body weight ratios.

Compared to the 5002 diet, uterine weight:body weight ratios were higher with the 5015 diet, NIH 31 diet, and the AIN-76A diet on Days 3 , 5 , and 7; no significant increases in uterine weight were noted for the 5001 or the NIH-07 diets compared to the 5002 diet.
Wade et al. (2003)
Mäkelä et

Thigpen et al. (1987, 1999) al. (1995b)

Birth Defects Res B Dev Reprod Toxicol. Author manuscript; available in PMC 2008 March 11. 


\begin{tabular}{|c|c|c|c|c|}
\hline Animal & Design & Endpoint(s) & Results & Reference \\
\hline $\begin{array}{l}\text { Adult cynomolgus } \\
\text { (Macaca } \\
\text { fascicularis) } \\
\text { monkey, } \\
\text { ovariectomized }\end{array}$ & $\begin{array}{l}\text { Monkeys }(\mathrm{n}=12) \text { were fed a } \\
\text { soy protein isolate diet } \\
\text { providing a dose of } 26.6 \mathrm{mg} \\
\text { free genistein/monkey/day (the } \\
\text { equivalent of a women } \\
\text { receiving } 99.7 \mathrm{mg} \text { genistein/ } \\
\text { day }[\sim 2 \mathrm{mg} / \mathbf{k g ~ b w} / \mathbf{d a y} \\
\left.\text { assuming a } 58 \mathbf{~ k g ~ b w}]^{b}\right) \text {. A } \\
\text { control group }(\mathrm{n}=13) \text { was } \\
\text { given isoflavone-extracted soy } \\
\text { diet and a positive control } \\
\text { group the extracted soy diet } \\
\text { supplemented with estrogen (n } \\
=15) \text {. Animals were fed the } \\
\text { diets for } 6 \text { months. }\end{array}$ & $\begin{array}{l}\text { Vaginal maturation and } \\
\text { karyopyknotic indices }\end{array}$ & $\begin{array}{l}\text { Not significantly affected by } \\
\text { soy diet }\end{array}$ & $\begin{array}{l}\text { Cline et al. } \\
(1996)\end{array}$ \\
\hline $\begin{array}{l}\text { Adult cynomolgus } \\
\text { (Macaca } \\
\text { fascicularis) } \\
\text { monkey, } \\
\text { ovariectomized }\end{array}$ & $\begin{array}{l}\text { For } 36 \text { months, monkeys }(\mathrm{n}= \\
57-62 / \text { group) were fed soy } \\
\text { protein isolate that was alcohol } \\
\text { treated to remove isoflavones } \\
\text { (negative control), untreated } \\
\text { soy protein isolate ( } 91 \mathrm{mg} \\
\text { genistein, } 31 \mathrm{mg} \text { daidzein, and } \\
7 \mathrm{mg} \text { glycitein), or alcohol- } \\
\text { extracted soy protein isolate } \\
\text { containing conjugated equine } \\
\text { estrogens (positive control). }\end{array}$ & $\begin{array}{l}\text { Breast and uterine } \\
\text { proliferation, sex steroid } \\
\text { receptor expression, and } \\
\text { serum estrogen level }\end{array}$ & $\begin{array}{l}\text { In the soy protein isolate } \\
\text { group, there was no increase } \\
\text { in breast or uterine } \\
\text { proliferation or steroid } \\
\text { receptor expression; } \\
\text { mammary gland thickness } \\
\text { and serum estrone and } 17 \beta \text { - } \\
\text { estradiol levels were } \\
\text { significantly reduced. }\end{array}$ & $\begin{array}{l}\text { Wood et al. } \\
(2004)\end{array}$ \\
\hline
\end{tabular}

${ }^{a}$ Because statistical significance was not clearly indicated, only obvious effects are listed.

$b$

${ }^{b}$ Assumptions used in dose estimates obtained from USEPA (1988). 
Table 20

Development of UDPGT Activity in Humans

\begin{tabular}{|c|c|c|c|}
\hline \multirow[b]{2}{*}{ Age } & \multicolumn{3}{|c|}{ UDPGT activity towards substrate $(\mathrm{nmol} / \mathrm{min} / \mathrm{mg} \text { protein })^{a}$} \\
\hline & Bilirubin & Testosterone & 1-Naptho \\
\hline $\begin{array}{l}30 \text { weeks gestation } \\
30 \text { weeks gestation with } 10 \text { weeks survival } \\
\text { Full-term infants surviving } 1-10 \text { days }(\mathrm{n}=7) \\
\text { Full-term infants surviving } 8-15 \text { weeks }(\mathrm{n}=6) \\
\text { Full-term infants surviving } 22-55 \text { weeks }(\mathrm{n}=5) \\
\text { Adult males }(\mathrm{n}=3)\end{array}$ & $\begin{array}{c}0.05 \\
0.4,1 \\
0.07 \pm 0.04 \\
0.64 \pm 0.32 \\
0.99 \pm 1.1 \\
0.76 \pm 0.43\end{array}$ & $\begin{array}{c}0 \\
0.14,0.85 \\
0.10 \pm 0.06 \\
0.12 \pm 0.05 \\
0.09 \pm 0.06 \\
0.46 \pm 0.61\end{array}$ & $\begin{array}{c}0.56 \\
3.0,1.8 \\
0.75 \pm 0.68 \\
2.4 \pm 1.1 \\
3.6 \pm 2.1 \\
7.2 \pm 2.2\end{array}$ \\
\hline
\end{tabular}

${ }^{a}$ Data presented as individual values or mean \pm SD. From Coughtrie et al. (1988). 
Table 21

Plasma Biochemical Values at 3 Months of Age in Children Given Soy Formula or Evaporated Cow-Milk Formula $^{a}$

\begin{tabular}{|c|c|c|c|}
\hline Laboratory test & Group (n) & Mean \pm SD & $P$ value by $t$-test \\
\hline Cholesterol, mg. \% & $\begin{array}{c}\text { Soy (14) } \\
\text { Cow milk (14) }\end{array}$ & $\begin{array}{l}126 \pm 34 \\
163 \pm 32\end{array}$ & 0.01 \\
\hline Total plasma protein, g \% & $\begin{array}{c}\text { Soy (14) } \\
\text { Cow milk (14) }\end{array}$ & $\begin{array}{l}6.7 \pm 0.8 \\
6.0 \pm 0.5\end{array}$ & 0.03 \\
\hline$\alpha 2-$ Globulin & $\begin{array}{c}\text { Soy (7) } \\
\text { Cow milk (8) }\end{array}$ & $\begin{array}{c}8 \pm 4 \\
14 \pm 5\end{array}$ & 0.01 \\
\hline$\gamma$-Globulin, $\%$ & $\begin{array}{c}\text { Soy (7) } \\
\text { Cow milk (8) }\end{array}$ & $\begin{array}{c}9 \pm 3 \\
15 \pm 4\end{array}$ & 0.001 \\
\hline
\end{tabular}

${ }^{a}$ From Kay et al. (1960). 
Table 22

Laboratory Values at 6 Months of Age ${ }^{a}$

\begin{tabular}{|c|c|c|c|}
\hline Analyte & Human milk $(n=8)$ & Enfamil $(n=11)$ & ProSobee $(n=11)$ \\
\hline \multicolumn{4}{|l|}{ Serum measurement } \\
\hline Vitamin D (ng/mL) & $2.2 \pm 0.9$ & $2.0 \pm 0.8$ & $2.3 \pm 1.2$ \\
\hline 25-Hydroxyvitamin D (ng/mL) & $25.6 \pm 3.3$ & $23.8 \pm 3.1$ & $24.0 \pm 5.3$ \\
\hline 1,25-Dihydroxyvitamin $\mathrm{D}(\mathrm{pg} / \mathrm{mL})$ & $29.3 \pm 11.3$ & $45.4 \pm 15.9^{b}$ & $47.7 \pm 10.2^{b}$ \\
\hline Calcium (mg/dL) & $10.0 \pm 0.6$ & $9.9 \pm 0.6^{c}$ & $10.5 \pm 0.5, b c$ \\
\hline Phosphorus (mg/dL) & $5.5 \pm 0.3$ & $6.0 \pm 0.7^{d}$ & $6.6 \pm 0.9^{b}$ \\
\hline Magnesium $(\mathrm{mEq} / \mathrm{L})$ & $1.81 \pm 0.13$ & $1.79 \pm 0.13^{c}$ & $1.95 \pm 0.15, b c$ \\
\hline Alkaline phosphatase (IU/L) & $78 \pm 22$ & $59 \pm 8$ & $70 \pm 23$ \\
\hline Parathyroid hormone $\left(\mu \mathrm{lEg} / \mathrm{mL}^{d}\right)$ & $3.2 \pm 2.5$ & $2.2 \pm 0.4$ & $3.1 \pm 0.8$ \\
\hline Albumin (g/dL) & $4.7 \pm 0.4$ & $4.5 \pm 0.3$ & $4.5 \pm 0.6$ \\
\hline \multicolumn{4}{|l|}{ Urine measurement } \\
\hline Calcium (mg/mg creatinine) & $0.37 \pm 0.31$ & $0.25 \pm 0.14$ & $0.29 \pm 0.28$ \\
\hline Phosphorus (mg/mg creatinine) & $0.71 \pm 0.44$ & $1.4 \pm 0.3^{b}$ & $1.5 \pm 0.66^{b}$ \\
\hline Magnesium (mEq/mg creatinine) & $0.018 \pm 0.008$ & $0.018 \pm 0.005^{c}$ & $0.029 \pm 0.017, b c$ \\
\hline
\end{tabular}

${ }^{a}$ Values are mean \pm SD. From Hillman et al. (1988).

${ }^{b}$ Different from human milk: $P<0.05$.

${ }^{c}$ Difference between ProSobee and Enfamil, $P<0.05$.

$d_{\text {Units as in the original. }}$ 
Table 23

Formula Composition $^{a}$

\begin{tabular}{llll}
\hline Component & Soy & Cow milk & Hypercaloric cow milk \\
\hline Energy, kcal/L & 680 & 680 & 810 \\
Protein, g/L & 20.0 & 15.5 & 22.0 \\
Fat, g/L & 36.0 & 36.1 & 44.9 \\
Calcium, mg/L & 700 & 510 & 730 \\
Phosphorous, mg/L & 500 & 390 & 560 \\
Vitamin D, IU/L & 400 & 400 & 480 \\
\hline
\end{tabular}

${ }^{a}$ From Kulkarni et al. (1984). 
Table 24

Mean IgG Antibody Levels to $\beta$-Lactoglobulin ${ }^{a}$

\begin{tabular}{llcl}
\hline & \multicolumn{2}{c}{ Formula } & \\
\cline { 2 - 3 } Age (months) & Soy & Cow milk & \multicolumn{1}{c}{ Not significant } \\
& & & $<0.01$ \\
Newborn & 26 & 38 & $<0.001$ \\
3 & 28 & 120 & $<0.05$ \\
9 & 24 & 355 & $<0.05$ \\
12 & 85 & 270 & Not significant \\
18 & 74 & 285 & \\
36 & 83 & 237 & \\
\hline
\end{tabular}

${ }^{a}$ Data given as mean $\%$ of a reference serum, estimated from a graph. The reference serum was not defined, variances were not shown, and the statistical methods were not given. From Kjellman and Johansson (1979). 
Table 25

Composition of Reconstituted Formulas ${ }^{a}$

\begin{tabular}{llcc}
\hline Protein (n) & $\begin{array}{l}\text { Protein concentration, } \mathbf{g} / \mathbf{1 0 0 m L} \\
\text { (\% kcal from protein) }\end{array}$ & Calories, kJ/100 $\mathbf{~ L L}$ & $\begin{array}{c}\text { Protein:calorie ratio, g protein/ } \\
\mathbf{1 0 0} \text { calories }\end{array}$ \\
\hline Cow-milk formula & & & \\
$2.0 \mathrm{~g} / \mathrm{kg} /$ day (7) & $1.6(8.6)$ & $74.2(310.4)$ & 2.2 \\
$\begin{array}{l}.0 \mathrm{~g} / \mathrm{kg} / \text { day (7) } \\
\text { Soy formula }\end{array}$ & $3.0(15.9)$ & $75.4(315.5)$ & 2.0 \\
$2.0 \mathrm{~g} / \mathrm{kg} /$ day (13) & $1.6(8.6)$ & $74.2(310.4)$ & 2.2 \\
$5.0 \mathrm{~g} / \mathrm{kg} /$ day (14) & $3.5(21.0)$ & $70.5(295.0)$ & 5.3 \\
\hline
\end{tabular}

${ }^{a}$ Per kg assumed to be per kg bw]. From Zoppi et al. (1982). 
Table 26

Factors Associated With Thelarche Before 2 Years of Age on Multivariate Analysis ${ }^{a}$

\begin{tabular}{llr}
\hline Factor & OR $(\mathbf{9 5 \%} \mathbf{C I})$ & $\boldsymbol{P}$ value (two-sided) \\
\hline Soy formula & $2.7(1.1-6.8)$ & 0.029 \\
Maternal ovarian cysts & $6.8(1.4-33.0)$ & 0.017 \\
Chicken consumption & $4.9(1.1-21.9)$ & 0.035 \\
Corn consumption & $0.2(0.0-0.9)$ & 0.039 \\
\hline
\end{tabular}

${ }^{a}$ From Freni-Titulaer (1986). 


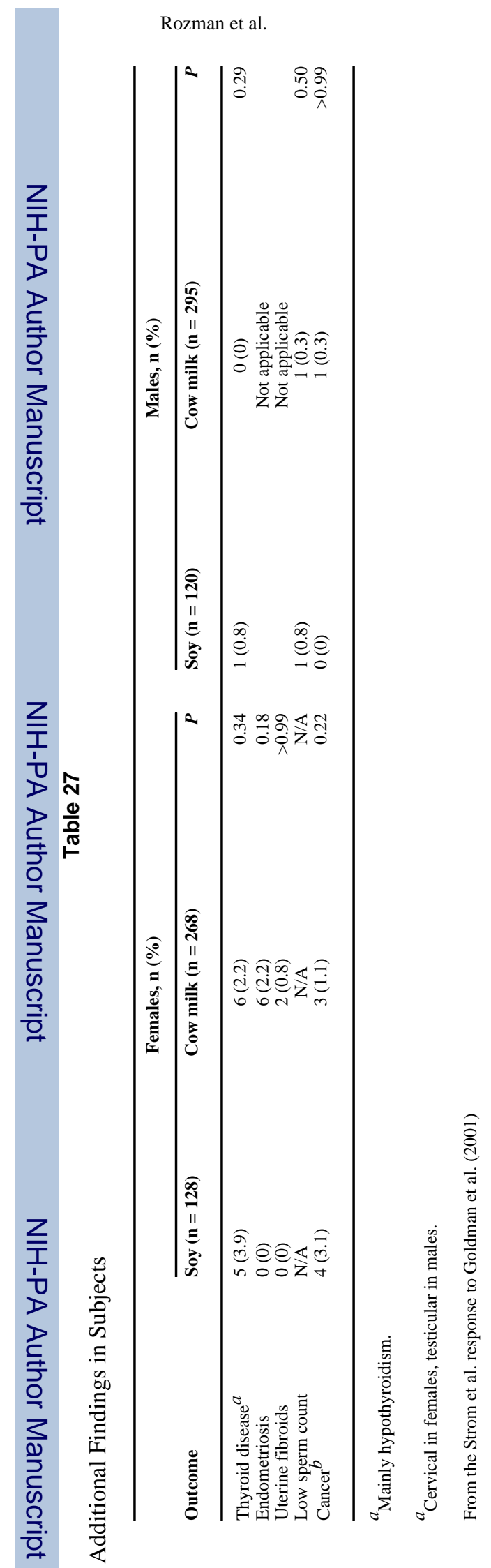

Page 188 


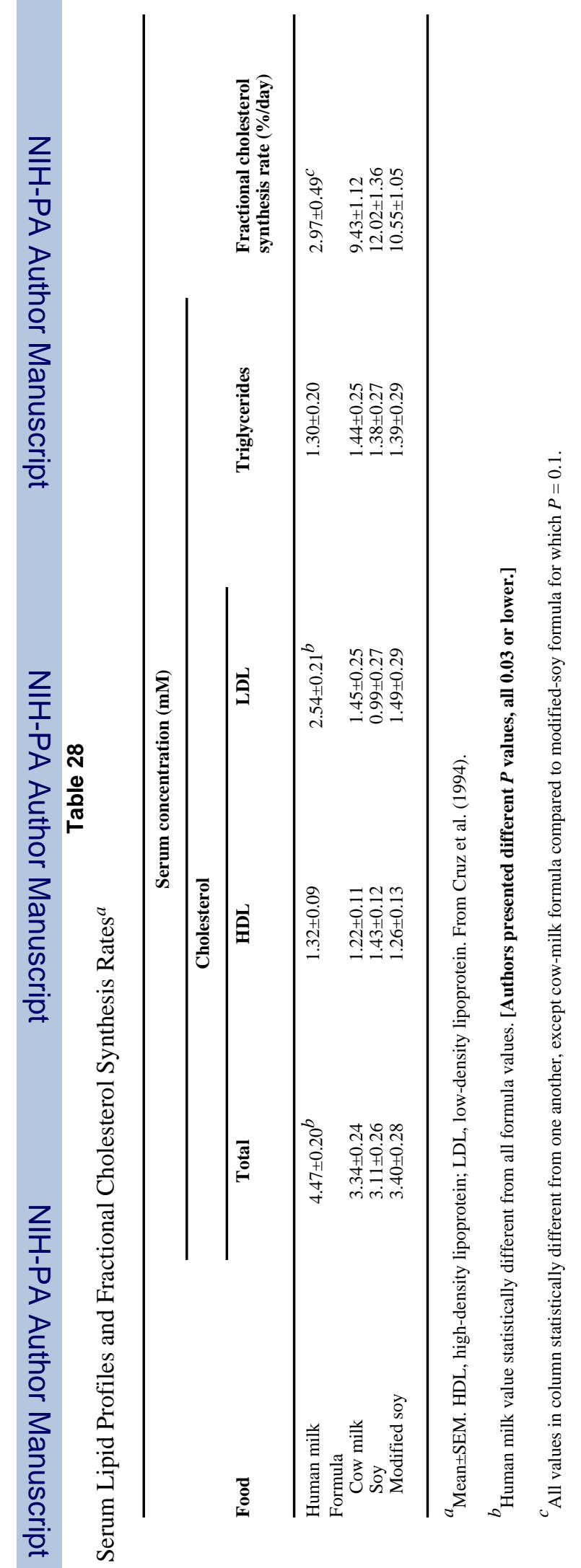


Table 29

Association Between Dietary Soy During Adolescence and Adult Breast Cancer

\begin{tabular}{lrr}
\hline Soy intake quintile (soy protein cut-off, g/day) & Adjusted OR (95\% CI) \\
\cline { 2 - 3 } & Subject recall \\
\hline $1(<2.20)$ & Reference & Meference \\
$2(2.20-4.40)$ & $0.75(0.60-0.93)$ & $0.70(0.45-1.09)$ \\
$3(4.41-6.60)$ & $0.69(0.55-0.87)$ & $0.59(0.32-1.07)$ \\
$4(6.61-11.00)$ & $0.69(0.55-0.86)$ & $0.60(0.37-0.99)$ \\
$5(\geq 11.01)$ & $0.51(0.40-0.65)$ & $0.35(0.21-0.60)$ \\
Trend test $P$ value & $<0.01$ & 0.0002 \\
\hline
\end{tabular}

${ }^{a}$ OR adjusted for intake of rice and wheat products, education, family history of breast cancer, history of fibroadenomas, waist:hip ratio, age at menarche, physical activity, ever giving birth, age at first delivery, menopausal status, and age at menopause.

From Shu et al. (2001). 
Table 30

Statistically Significant Relationships Between Soy Intake and Breast Cancer ${ }^{a}$

\begin{tabular}{|c|c|c|c|c|}
\hline \multirow[b]{2}{*}{ Exposure Group } & \multicolumn{2}{|c|}{ Subjects, n } & \multicolumn{2}{|c|}{ Adjusted OR (95\% CI) } \\
\hline & Cases & Controls & $\bar{b}$ & $c$ \\
\hline Tofu intake of 1-3 times/week in adolescence & 189 & 270 & $0.56(0.38-0.82)$ & $0.62(0.42-0.92)$ \\
\hline Tofu intake of $\geq 4$ times/week in adolescence & 55 & 85 & $0.51(0.31-0.84)$ & $0.65(0.38-1.10)$ \\
\hline $\begin{array}{l}\text { Intake of }>12.68 \mathrm{mg} \text { isoflavone } / 1,000 \mathrm{kcal} \text { in } \\
\text { adulthood }\end{array}$ & 104 & 166 & $0.51(0.33-0.78)$ & $0.61(0.39-0.97)$ \\
\hline $\begin{array}{l}\text { High tofu intake in adolescence }(\geq 1 \text { time/week } \\
\text { intake) and high isoflavone intake }(>6.24 \mathrm{mg} \\
\text { isoflavone/1000 kcal in adulthood })\end{array}$ & 164 & 261 & $0.53(0.36-0.78)$ & $0.65(0.43-0.97)$ \\
\hline
\end{tabular}

$a_{\text {From Wu et al. (2002) }}$

${ }^{b}$ Adjusted for birthplace, education, age at menarche, parity, current body-mass index, menopausal status, hormone use.

${ }^{c}$ Further adjusted for dark leafy green intake in adolescence, smoking, alcohol ingestion, physical activity, and family history of breast cancer. 
Table 31

Effects of Diet and Soy Formula Given to Rats on PND 21-50

\begin{tabular}{|c|c|c|c|c|}
\hline \multirow[b]{2}{*}{ Endpoint } & \multicolumn{2}{|c|}{ Experiment 1} & \multicolumn{2}{|c|}{ Experiment 2} \\
\hline & RM1 diet (control) & AIN-76A diet & RM1 diet (control) & $\begin{array}{c}\text { RM1 diet + } \\
\text { Infasoy }\end{array}$ \\
\hline \multicolumn{5}{|l|}{ Body weight (g) } \\
\hline PND 21 & $40.8 \pm 4.7$ & $40.2 \pm 3.7$ & $37.1 \pm 5.7$ & $37.3 \pm 5.6$ \\
\hline PND 35 & $107.1 \pm 9.1$ & $120.5 \pm 8.3^{b}$ & $106.0 \pm 12.0$ & $107.6 \pm 17.0$ \\
\hline PND 46 & Not determined & Not determined & $156.2 \pm 12.3$ & $165.3 \pm 14.4^{b}$ \\
\hline At vaginal opening & $108.6 \pm 11.6$ & $97.7 \pm 9.0^{b}$ & $102.4 \pm 13.2$ & $91.5 \pm 10.8^{b}$ \\
\hline At first estrus & Not determined & Not determined & $115.2 \pm 19.8$ & $108.3 \pm 18.7$ \\
\hline \multicolumn{5}{|l|}{ Age (days) } \\
\hline At vaginal opening & $35.1 \pm 1.9$ & $31.5 \pm 1.2^{b}$ & $34.5 \pm 2.0$ & $32.4 \pm 1.2^{b}$ \\
\hline At first estrus & $38.1 \pm 2.8$ & $34.6 \pm 3.5^{b}$ & $37.1 \pm 3.9$ & $35.3 \pm 2.7^{c}$ \\
\hline
\end{tabular}

${ }^{a}$ From Ashby et al. (2000b). Data expressed as mean \pm variance [unspecified but listed as SD in other figures of this report].

${ }^{b} P<0.01$.

${ }^{c} P<0.05$ compared to control. 


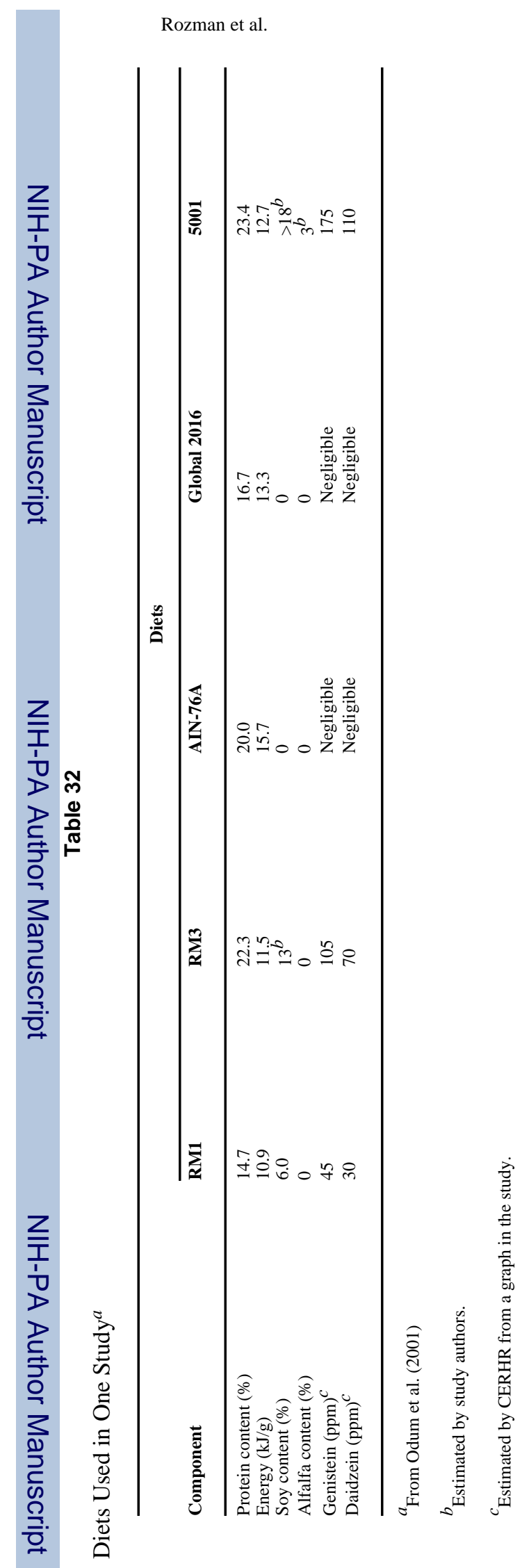

Page 193 
Table 33

Effects of Diet on Pregnancy and Lactation in Rats ${ }^{a}$

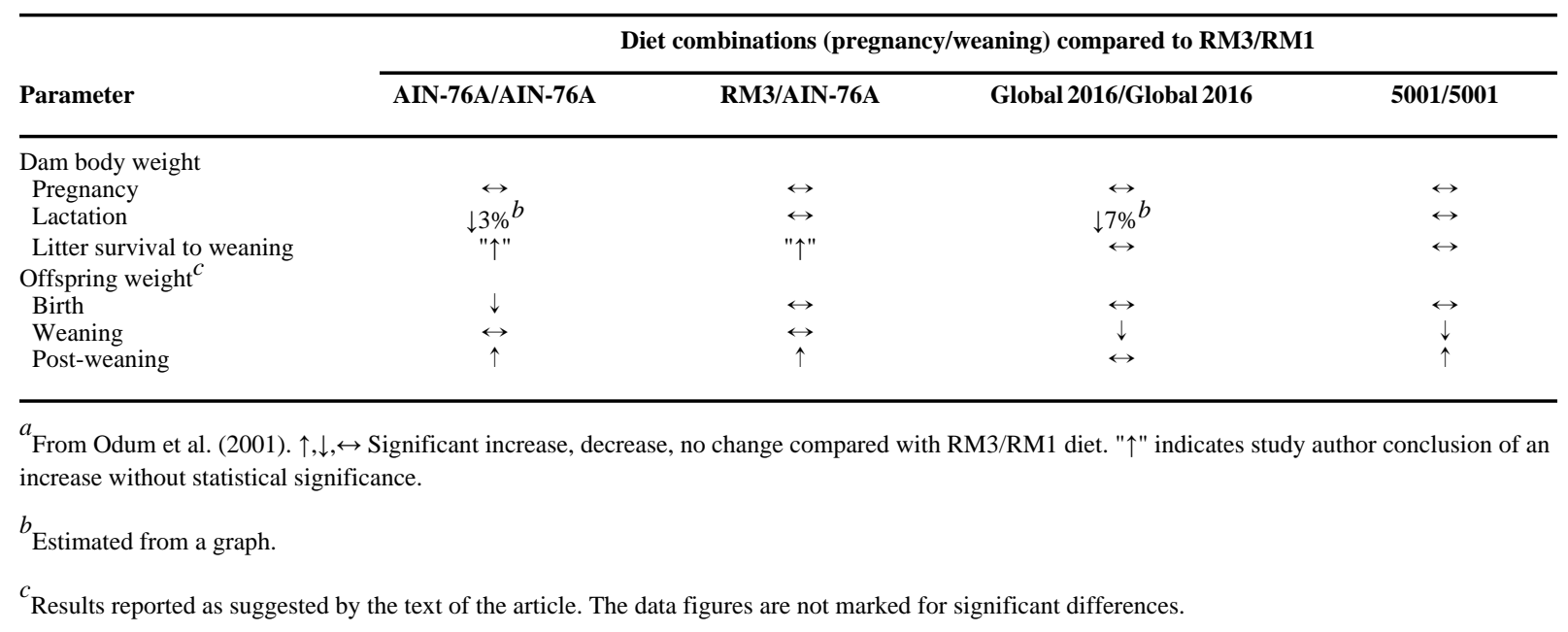


Table 34

Effects of Diet on Rat Developmental Landmarks and Reproductive Organ Weights ${ }^{a}$

\begin{tabular}{|c|c|c|c|c|}
\hline \multirow[b]{2}{*}{ Parameter } & \multicolumn{4}{|c|}{ Diet Combinations (pregnancy/weaning) Compared to RM3/RM1 } \\
\hline & AIN-76A/AIN-76A & RM3/AIN-76A & $\begin{array}{l}\text { Global 2016/ } \\
\text { Global 2016 }\end{array}$ & $5001 / 5001$ \\
\hline \multicolumn{5}{|l|}{ Testis descent } \\
\hline Age & $\uparrow 3 \%$ & $\downarrow 3 \%$ & $\leftrightarrow$ & $\leftrightarrow$ \\
\hline Body weight & $\leftrightarrow$ & $\leftrightarrow$ & $\downarrow 8 \%$ & $\leftrightarrow$ \\
\hline \multicolumn{5}{|l|}{ Preputial separation } \\
\hline Age & $\downarrow 5 \%$ & $\downarrow 7 \%$ & $\leftrightarrow$ & $\downarrow 4 \%$ \\
\hline Body weight & $\leftrightarrow$ & $\leftrightarrow$ & $\leftrightarrow$ & $\leftrightarrow$ \\
\hline \multicolumn{5}{|l|}{ Vaginal opening } \\
\hline Age at onset & $\downarrow 7 \%$ & $\downarrow 10 \%$ & $\leftrightarrow$ & $\leftrightarrow$ \\
\hline Body weight at completion & $\leftrightarrow$ & $\downarrow 12 \%$ & $\downarrow 11 \%$ & $\leftrightarrow$ \\
\hline Age at first estrus & $\leftrightarrow$ & $\downarrow 11 \%$ & $\leftrightarrow$ & $\downarrow 8 \%$ \\
\hline \multirow{2}{*}{\multicolumn{5}{|c|}{ Body weight (adjusted for weaning weight) and relative organ weight) }} \\
\hline \multicolumn{3}{|c|}{ Females on PND 26} & & \\
\hline Body weight & $\uparrow 10 \%$ & $\uparrow 11 \%$ & $\downarrow 5 \%$ & $\uparrow 14 \%$ \\
\hline Uterus (blotted) & $\uparrow 37 \%$ & $\uparrow 83 \%$ & $\uparrow 28 \%$ & $\uparrow 77 \%$ \\
\hline Uterus (dry) & $\uparrow 30 \%$ & $\uparrow 78 \%$ & $\uparrow 24 \%$ & $\uparrow 70 \%$ \\
\hline Vagina & $\uparrow 17 \%$ & $\uparrow 42 \%$ & $\uparrow 16 \%$ & $\uparrow 27 \%$ \\
\hline Cervix & $\leftrightarrow$ & $\uparrow 45 \%$ & $\leftrightarrow$ & $\leftrightarrow$ \\
\hline Ovary & $\leftrightarrow$ & $\uparrow 11 \%$ & $\leftrightarrow$ & $\leftrightarrow$ \\
\hline \multicolumn{5}{|l|}{ Males on PND 68} \\
\hline Body weight & $\uparrow 16 \%$ & $\uparrow 9 \%$ & $\leftrightarrow$ & $\uparrow 13 \%$ \\
\hline Liver & $\uparrow 12 \%$ & $\uparrow 10 \%$ & $\leftrightarrow$ & $\leftrightarrow$ \\
\hline Kidney & $\uparrow 7 \%$ & $\uparrow 26 \%$ & $\leftrightarrow$ & $\uparrow 22 \%$ \\
\hline Testis & $\downarrow 10 \%$ & $\leftrightarrow$ & $\leftrightarrow$ & $\leftrightarrow$ \\
\hline Epididymis & $\downarrow 9 \%$ & $\leftrightarrow$ & $\leftrightarrow$ & $\leftrightarrow$ \\
\hline Seminal vesicle & $\leftrightarrow$ & $\leftrightarrow$ & $\leftrightarrow$ & $\leftrightarrow$ \\
\hline \multirow{2}{*}{\multicolumn{5}{|c|}{ Males on PND 128}} \\
\hline & & & & \\
\hline Body weight & $\uparrow 10 \%$ & $\uparrow 14 \%$ & $\leftrightarrow$ & $\leftrightarrow$ \\
\hline Liver & $\leftrightarrow$ & $\leftrightarrow$ & $\leftrightarrow$ & $\leftrightarrow$ \\
\hline Kidney & $\leftrightarrow$ & $\leftrightarrow$ & $\leftrightarrow$ & $\uparrow 9 \%$ \\
\hline Testis & $\downarrow 9 \%$ & $\leftrightarrow$ & $\leftrightarrow$ & $\leftrightarrow$ \\
\hline Epididymis & $\leftrightarrow$ & $\leftrightarrow$ & $\leftrightarrow$ & $\leftrightarrow$ \\
\hline Seminal vesicle & $\leftrightarrow$ & $\leftrightarrow$ & $\leftrightarrow$ & $\leftrightarrow$ \\
\hline Prostate & $\leftrightarrow$ & $\leftrightarrow$ & $\leftrightarrow$ & $\leftrightarrow$ \\
\hline \multicolumn{5}{|l|}{ Females on PND 140-144 } \\
\hline Body weight & $\uparrow 9 \%$ & $\uparrow 13 \%$ & $\leftrightarrow$ & $\leftrightarrow$ \\
\hline Liver & $\leftrightarrow$ & $\leftrightarrow$ & $\leftrightarrow$ & $\leftrightarrow$ \\
\hline Kidney & $\uparrow 22 \%$ & $\uparrow 22 \%$ & $\leftrightarrow$ & $\leftrightarrow$ \\
\hline Uterus (blotted) & $\leftrightarrow$ & $\leftrightarrow$ & $\leftrightarrow$ & $\leftrightarrow$ \\
\hline Uterus (dry) & $\downarrow 12 \%$ & $\leftrightarrow$ & $\leftrightarrow$ & $\leftrightarrow$ \\
\hline Vagina & $\leftrightarrow$ & $\leftrightarrow$ & $\leftrightarrow$ & $\leftrightarrow$ \\
\hline Cervix & $\leftrightarrow$ & $\leftrightarrow$ & $\leftrightarrow$ & $\leftrightarrow$ \\
\hline Ovary & $\leftrightarrow$ & $\leftrightarrow$ & $\leftrightarrow$ & $\leftrightarrow$ \\
\hline
\end{tabular}

PND, postnatal day.

$a_{\mathrm{a} \uparrow, \downarrow, \leftrightarrow}$ Significantly increased (older), decreased (younger), unchanged compared with RM3/RM1 diet.

From Odum et al. (2001). 


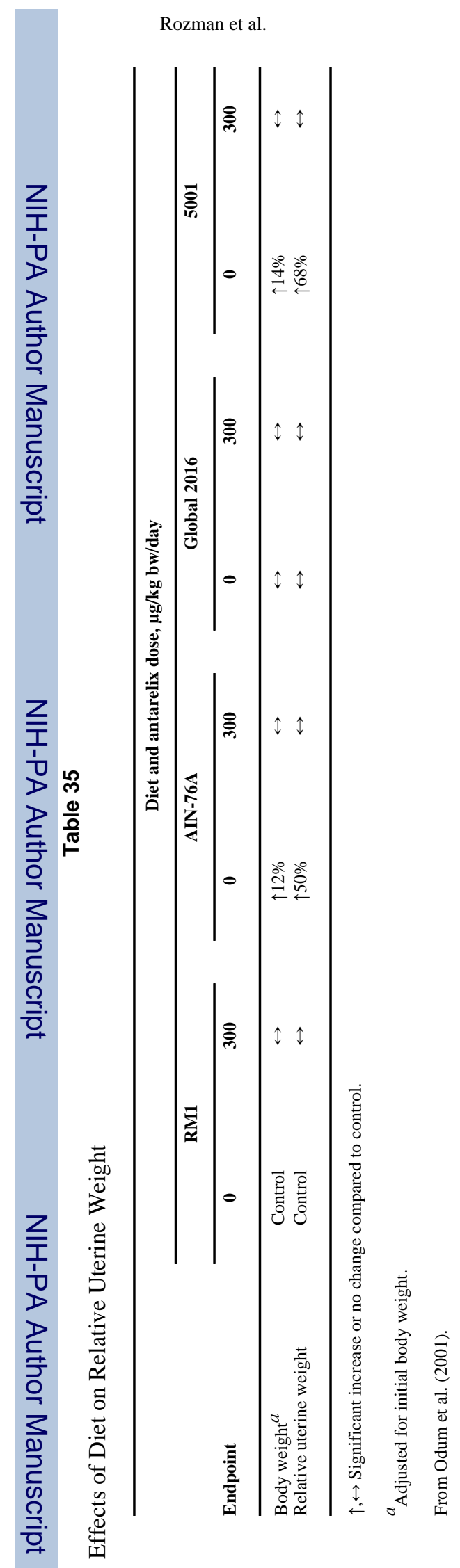

Page 196 


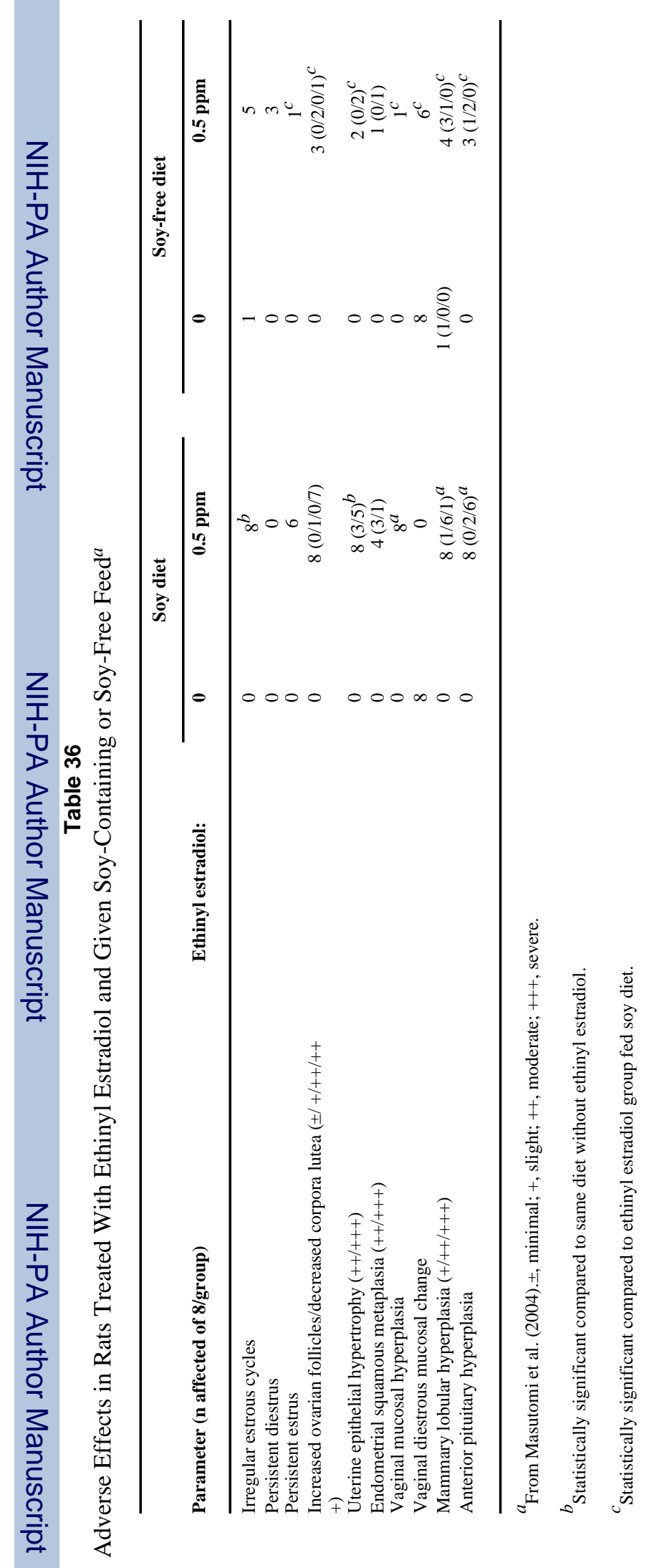

Birth Defects Res B Dev Reprod Toxicol. Author manuscript; available in PMC 2008 March 11. 
Table 37

Effects of Neonatal Exposures to Soy-Free Diet or Genistein on the Reproductive System of Male Rats ${ }^{a}$

\begin{tabular}{lcc}
\hline Effect & $\begin{array}{c}\text { Soy-free control compared to } \\
\text { standard-diet control }\end{array}$ & $\begin{array}{c}\text { Genistein } 4 \text { mg/kg bw/day (soy- } \\
\text { free diet) compared to soy-free } \\
\text { control }\end{array}$
\end{tabular}

Germ cell apoptotic index, PND 18

Germ cell apoptotic index, PND 25

Seminiferous tubule lumen formation, PND 18

Plasma inhibin B, PND 18

Sertoli cell nuclear volume/testis, PND 18

Plasma FSH, PND 18

Plasma FSH, PND 25

Spermatocyte/Sertoli cell nuclear volume, PND 18

Spermatocyte/Sertoli cell nuclear volume, PND 25

$\hat{\imath}$

PND, postnatal day.

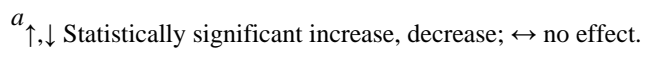

From Atanassova et al. (2000). 


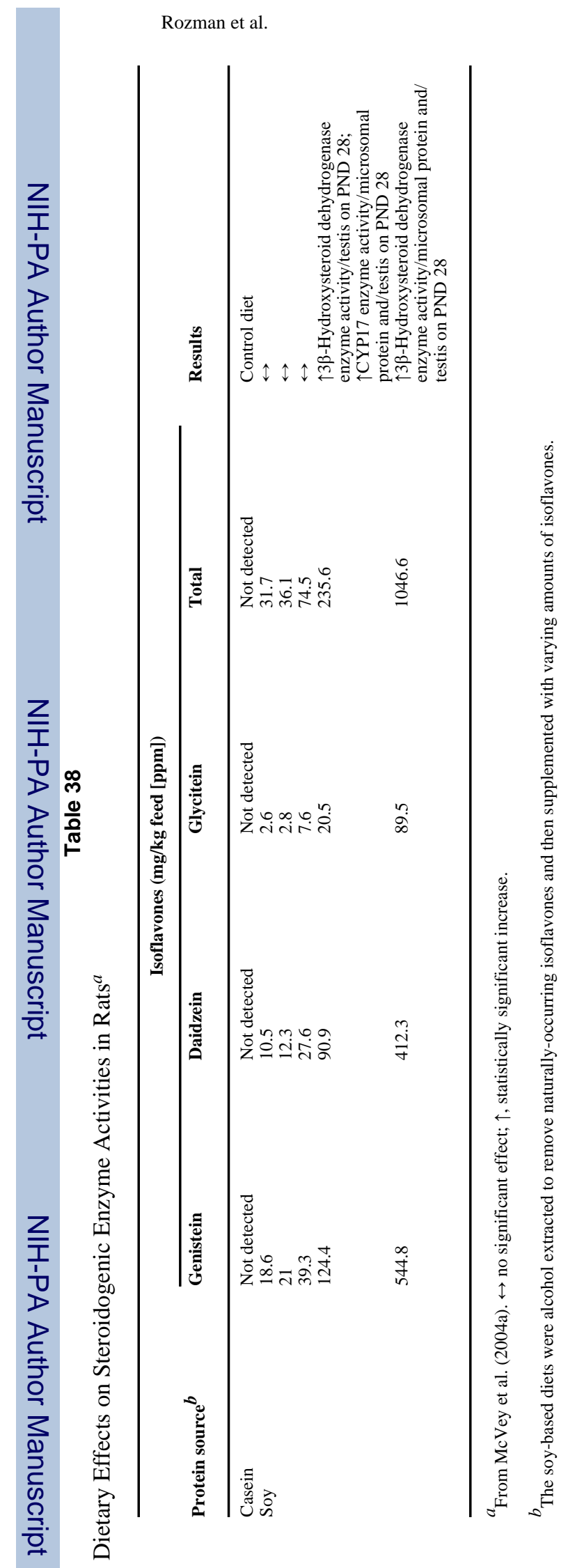

Page 199

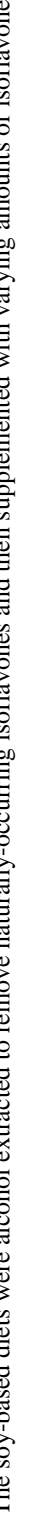


Table 39

Summary of Significant Effects in Wild-Type and Aromatase-Knockout Mice Fed Diets With and Without Soy $^{a}$

\begin{tabular}{|c|c|c|c|c|}
\hline \multirow[b]{2}{*}{ Parameter } & \multicolumn{4}{|c|}{ Genotype and dietary comparisons $b$} \\
\hline & $\begin{array}{c}\text { KO-soy }^{+} \text {vs. } \\
\text { KO-soy }^{-}\end{array}$ & $\begin{array}{c}\text { KO-soy }^{+} \text {vs. } \\
\text { WT-soy }^{+}\end{array}$ & $\begin{array}{c}\text { KO-soy }^{-} \text {vs. } \\
\text { WT-soy }^{-}\end{array}$ & $\begin{array}{c}\text { WT-soy }^{+} \text {vs. } \\
\text { WT-soy }\end{array}$ \\
\hline \multicolumn{5}{|l|}{14 weeks of age } \\
\hline Seminiferous tubule diameter & $\uparrow 13 \%$ & $\leftrightarrow$ & $\leftrightarrow$ & $\uparrow 8.3 \%$ \\
\hline Seminiferous tubule length & $\downarrow 12 \%$ & $\leftrightarrow$ & $\leftrightarrow$ & $\downarrow 13 \%$ \\
\hline Testicular spermatogonia numbers & $\downarrow 29 \%$ & $\downarrow 29 \%$ & $\leftrightarrow$ & $\leftrightarrow$ \\
\hline Testicular round spermatid numbers & $\uparrow 22 \%$ & $\leftrightarrow$ & $\leftrightarrow$ & $\leftrightarrow$ \\
\hline Sertoli cell numbers & $\leftrightarrow$ & $\uparrow 7.7 \%$ & $\leftrightarrow$ & $\downarrow 7.1 \%$ \\
\hline \multicolumn{5}{|l|}{1 year of age } \\
\hline Seminiferous tubule lumen volume & $\uparrow 550 \%$ & $\leftrightarrow$ & $\downarrow 80 \%$ & $\leftrightarrow$ \\
\hline Testicular epithelial volume & $\uparrow 160 \%$ & $\downarrow 32 \%$ & $\downarrow 79 \%$ & $\downarrow 21 \%$ \\
\hline Testicular intersitial volume & $\leftrightarrow$ & $\leftrightarrow$ & $\uparrow 109 \%$ & $\uparrow 45 \%$ \\
\hline Seminiferous tubule diameter & $\uparrow 31 \%$ & $\leftrightarrow$ & $\downarrow 33 \%$ & $\leftrightarrow$ \\
\hline Seminiferous tubule length & $\leftrightarrow$ & $\leftrightarrow$ & $\downarrow 50 \%$ & $\downarrow 14 \%$ \\
\hline Testicular spermatocyte numbers & $\uparrow 317 \%$ & $\leftrightarrow$ & $\downarrow 81 \%$ & $\leftrightarrow$ \\
\hline Testicular round spermatid numbers & $\uparrow 360 \%$ & $\downarrow 54 \%$ & $\downarrow 90 \%$ & $\leftrightarrow$ \\
\hline Testicular elongated spermatid numbers & $\uparrow 400 \%$ & $\downarrow 56 \%$ & $\downarrow 92 \%$ & $\leftrightarrow$ \\
\hline Total germ cell numbers/Sertoli cell numbers & $\uparrow 240 \%$ & $\downarrow 55 \%$ & $\downarrow 86 \%$ & $\leftrightarrow$ \\
\hline
\end{tabular}

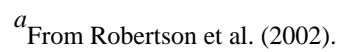

${ }^{b}$ Percent changes were estimated from a graph by CERHR.

$\uparrow, \downarrow, \leftrightarrow$ Significantly increased, decreased; KO aromatase-knockout mice; WT, wild-type mice; soy ${ }^{+}$, soy-containing diet, soy ${ }^{-}$, soy-free diet. 
Table 40

Effect of Diet on Dimethylbenzanthracene-Induced Mammary Tumors in Female Rats ${ }^{a}$

\begin{tabular}{|c|c|c|c|c|}
\hline \multirow[b]{2}{*}{ Parameter } & \multirow[b]{2}{*}{ Generation } & \multicolumn{3}{|c|}{ Diet } \\
\hline & & Casein & Soy & Whey \\
\hline \multicolumn{5}{|l|}{ Days after dimethylbenzanthracene } \\
\hline \multirow[t]{2}{*}{ To first tumor } & $\mathrm{F}_{1}$ & 28 & 42 & 28 \\
\hline & $\mathrm{F}_{2}$ & 36 & 36 & 43 \\
\hline \multirow[t]{2}{*}{ To $50 \%$ of rats with $\geq 1$ tumor } & $\mathrm{F}_{1}$ & $52^{b}$ & $65^{c}$ & $83^{c}$ \\
\hline & $\mathrm{F}_{2}$ & $54^{b}$ & $68^{c}$ & $89^{d}$ \\
\hline \multirow[t]{2}{*}{ Rats with at least 1 mammary tumor at end of study, $\%$} & $\mathrm{~F}_{1}$ & $100^{b}$ & $84,^{b c}$ & $62^{c}$ \\
\hline & $\mathrm{F}_{2}$ & $100^{b}$ & $77^{c}$ & $54^{c}$ \\
\hline \multirow[t]{2}{*}{ Rats with at least 1 adenocarcinoma, $\%$} & $\mathrm{~F}_{1}$ & 95 & 90 & 86 \\
\hline & $\mathrm{F}_{2}$ & 85 & 79 & 63 \\
\hline \multirow{2}{*}{$\begin{array}{l}\text { Rats with at least } 1 \text { mammary tumor with intraductal } \\
\text { proliferation, } \%\end{array}$} & $\mathrm{~F}_{1}$ & 5 & 5 & 5 \\
\hline & $\mathrm{F}_{2}$ & 13 & 21 & 32 \\
\hline \multirow[t]{2}{*}{ Tumors/rat, median (range) } & $\mathrm{F}_{1}$ & $5(2-12)^{b}$ & $3(1-12), b c$ & $2(1-22), c d$ \\
\hline & $\mathrm{F}_{2}$ & $3(1-7)^{b}$ & $3(1-10)^{b}$ & $2(1-7)^{c}$ \\
\hline \multirow[t]{2}{*}{ Median tumor volume, $\mathrm{cm}^{3}$} & $\mathrm{~F}_{1}$ & 1.9 & 1.5 & 0.6 \\
\hline & $\mathrm{F}_{2}$ & 3.0 & 1.8 & 0.8 \\
\hline
\end{tabular}

${ }^{a}$ From Hakkak et al. (2000).

${ }^{b-d}$ Within each row, numbers with different superscripts are statistically different from one another. 
Table 41

Studies in Humans Exposed as Infants to Soy Formula

Comparison groups
Growth and nutrition
Infants fed soy formula $(\mathrm{n}=14)$ and infants fed evaporated
cow-milk formula $(\mathrm{n}=14)$ from 24 hours to 3 months of age.

Infants fed soy infant formula $(\mathrm{n}=28)$ and infants fed cow-
milk formula $(\mathrm{n}=30)$ for 6 months. The soy formula was not
methionine-supplemented as is modern soy formula.

Infants fed soy infant formula $(\mathrm{n}=26)$, infants fed cow-milk formula $(n=29)$, and breast-fed infants $(n=26)$. Over the 6month study, many breast-fed infants dropped out or were given cow-milk formula.

Infants on soy formula $(\mathrm{n}=20)$ and infants on cow-milk formula $(n=20)$ for 16 weeks.

Infants on soy formula $(\mathrm{n}=13)$, infants on cow-milk formula $(n=20)$, and breast-fed infants $(n=26)$. Infants selected at 6 weeks of age and followed for 1 year.

Infants fed soy formula $(\mathrm{n}=18)$ or cow-milk formula $(\mathrm{n}=17)$ for 12 months.

Infants on soy formula $(\mathrm{n}=11)$, cow-milk formula $(\mathrm{n}=11)$, or human milk $(n=9)$, followed for 1 year.

Infants on soy formula with $(n=73)$ or without $(n=73)$ supplemental nucleotides, and infants breast-fed for 2 months and then given cow-milk formula $(n=67)$; infants followed to 12 months of age.

Very low birth-weight infants given soy formula, cow-milk formula, or high-calorie cow-milk formula until 3-4 months of age.

Preterm infants given soy formula or cow-milk formula $(\mathrm{n}=$ 40 overall), with change in soy-formula regimen after 10 infants were enrolled

Infants randomized to 1 of 2 different soy formulas $(n=10 /$ group) and 10 breast-fed infants (for comparisons to 4 months of age).

Infants exclusively fed soy formula $(\mathrm{n}=20)$, cow-milk formula $(n=19)$, or breast-fed $(n=17)$ for 4 months and followed until 6 months of age.

Infants given 1 of 2 different soy formulas ( $n=21$ /group), infants given cow-milk formula $(\mathrm{n}=20)$, and breast-fed infants $(n=10)$, followed for 1 year; solid food introduced at 3 months in breast-fed and 4 months in formula-fed groups.

Infants given a soy or cow-milk formula as subjects in 1 ofseveral feeding studies over a 14-year period; contacted as adults $20-32$ years of age.

Gastrointestinal effects

Infants with soy protein-induced intestinal villous atrophy (n $=2$ ) and normal infant $(n=1)$.

Infants with enterocolitis suspected due to cow milk- or soyprotein sensitivity, challenged with cow-milk and soy formulas. Allergy and immunology

Infants with a family history of major allergy assigned to soyformula $(n=79)$ or cow-milk formula $(n=201)$, followed 17 years; 48 infants with a family history of major allergy were breast-fed.

Infants with family history of allergic disease were permitted to be breast fed; an intervention group $(n=238)$ was told to avoid cow milk and was given soy formula and a non-intervention group $(n=249)$ was given no instruction; most of the latter infants were exposed to cow-milk formula; 1 year follow-up. Infants with cow milk allergy diagnosed at age 2-11 months were randomly assigned to extensively hydrolyzed formula (n $=90)$ orsoy formula $(n=80)$ and followed until age 2 years.
Major findings

Reference

Growth in both groups paralleled Iowa Growth charts. Infants on soy formula had lower cholesterol and higher total plasma protein.

Soy-fed infants, especially girls, lagged in volume consumed and growth from 2 to 4 months of age; cow-milk fed group gained more weight per ounce of formula.

Infants in all groups followed their centile growth curves.

Unable to detect a difference between the 2 groups in growth parameters, hematology measures, or blood chemistry.

Unable to detect growth differences between groups after 6 weeks of age; soy-fed infants showed slower bone mineralization at 3 months but not thereafter. Unable to detect differences in energy intake or growth; bone mineral content less in soy formula-fed infants.

Unable to detect a difference in bone width or bone mineral content; differences in serum and urine minerals and bone-related hormones were consistent with homeostatic adjustments to different mineral content in the food sources.

Groups were not comparable in weight and length at birth. No group differences in growth were detected after 6 months of age.

After 3 weeks of age, soy-fed infants had lower serum phosphorus and higher serum alkaline phosphatase.

Infants on soy formula gained less weight than infants on cow-milk formula.

Bone mineral density and plasma zinc higher in breast-fed than soy formula-fed infants at 4 months of age; unable to detect difference between soy formula-fed infants and historical controls in these parameters at 6 and 12 months.

Unable to detect group differences in growth and serum measurements related to bone accretion. Bone density was greater in soy formula-fed than breast-fed infants.

Unable to detect effect of feeding type on weight and head circumference; length lower in breast- than formula-fed groups; serum 1,25-dihydroxyvitamin D higher in one of the soy-formula groups.

Significant association between adult overweight and having been fed a soy formula as an infant (adjusted OR 1.47 ; 95\% CI 1.01-2.13).

Soy-sensitive infants had histologic intestinal changes similarto those seen with gluten enteropathy. Unable to detect differences in infant reactions to formula type. Infants often reacted to both.

Unable to detect a difference in development of allergic disease by formula type; breast-fed children were less likely to develop allergy after 3 years of age.

Neither formula offered a detectable advantage in preventing allergic disease; infants breast-fed for some period of time experienced protection from allergic disease compared to infants who were never breast-fed.

Parents suspected adverse reaction in $28 \%$ of subjects on soy formula and $11 \%$ of subjects on extensively hydrolyzed formula. Among children younger than 6 months, $40 \%$ were suspected by parents of having adverse reaction to soy formula.
Kay et al. (1960)

Cherry et al. (1968)

Dean (1973)

Jung and Carr (1977)

Köhler et al. (1984)

Steichen and Tsang (1987)

Hillman et al. (1988)

Lasekan et al. (1999)

Kulkarni et al. (1984)

Naude et al. (1979)

Chan et al. (1987)

Venkataraman (1992)

Mimouni et al. (1993)

Stettler et al. (2005)

Poley and Klein (1983)

Burks et al. (1994)

Gruskay (1982)

Miskelly et al. (1988)

Klemola et al. (2002) 
Infants with family history of atopy and whose mothers decided not to breast feed were randomized to soy formula $(n=41)$, cowmilk formula $(n=40)$, or casein-hydrolysate formula $(n=43)$ for 6 months; followed up to5 years with comparison to breastfed infants.

Children with peanut allergy $(n=49)$, children with atopy $(n=$ $70)$, and non-allergic children $(n=140)$ surveyed for infant feeding history.

Infants given 1 of 3 soy formulas, each with a different proteincontent $(\mathrm{n}=39$ overall) for 4 months.

Infants given 1 of 2 soy formulas ( $n=13$ or 14 /formula) or 1 of 2 cow-milk formulas ( $n=7 /$ formula), differing in protein content, from birth to 4.5 months of age.

Infants given soy formula $(\mathrm{n}=9), 1$ of 3 cow-milk formulas ( $\mathrm{n}$ $=7-10 /$ formula), or who were breast-fed $(n=27)$ for 5 months, after which all infants were switched to the same diet for the balance of the first year of life.

Infants fed cow-milk formula, soy formula, or a mixture of cow-milk and soy formulas $(n=67$ overall) and 7 breast-fed infants; follow-up was nearly 4 years.

Infants with clinical gastroenteritis that continued after switching to soy formula $(\mathrm{n}=18)$ were challenged with different foods.

Children with autoimmune thyroid disease $(n=59)$, healthy siblings $(n=76)$, and healthy unrelated controls $(n=54)$; history of infant feeding was evaluated.

Infants given 1 of 2 different soy formulas (n-92, 94), one of which contained added nucleotides, and breast-fed children who were weaned to cow-milk formula at 2 months $(n=81)$; 1 -year follow-up.

Thyroid function

Children with goiter related to soy formula-feeding $(n=3)$.

Infant with congenital hypothyroidism who was fed soy formula $(\mathrm{n}=1)$.

Infants with congenital hypothyroidism who were fed soy formula $(n=3)$.

Reproductive endpoints

Girls with premature thelarche $(n=130)$ and age-matched controlsubjects, retrospective questioning of parents about infant feeding.

Adults who had been fed soy $(\mathrm{n}=248)$ or cow-milk formula $(n=563)$ during infancy as part of a controlled trial; interviewed at 20-34 years of age

Children age 7-96 months who had been fed soy formula $(\mathrm{n}=$ 48 ) and children age 12-96 months who had not been fed soy formula $(\mathrm{n}=18)$.

Other endpoints

Infants on soy formula ( $\mathrm{n}=16$, some with cholesterol added), cow-milk formula $(n=10)$, or breast-fed with supplemental cow-milk formula $(n=12)$.

Diabetic children $(\mathrm{n}=95)$, non-diabetic siblings $(\mathrm{n}=194)$, and non-diabetic friends $(\mathrm{n}=95)$; infant feeding history obtained.
Breast-feeding offered an advantage in protection against atopic disease. Among the formula-fed infants, casein-hydrolysate formula was superior to either soy or cow-milk formula in protection against atopy.

[The Expert Panel is aware that the validity of these studies has been challenged.]

Soy consumption was an independent risk factor for peanut allergy (adjusted $\mathrm{OR}=2.61 ; 95 \% \mathrm{CI}=1.31$ 5.20)

Blood IgG levels were higher with the high-protein formula.

No detectable effect of formula type on head circumference andweight; serum cholesterol and C3 complement were lower in soy- than cow milkformula groups. Immunoglobulins were lower in lowprotein soy group than other groups.

Response to immunization was greatest in breast-fed infants and infants given a high-protein cow-milk formula, with the exception of antibody to polio, which was lower in breast-fed children than in formula-fed children. Soy formula-fed infants had more episodes of infection than breast-fed children. Serum from soy-fed infants bound soy protein less than serum from cow milk-fed infants bound cowmilk protein; feeding a soy product resulted in comparable or greater antibody response to cow milk than feeding a cow-milk formula.

Infants with positive challenges to soy and egg had higher anti-soy IgG than infants with a negative response.

Significantly more children with thyroid disease (31\%) received soy formula compared to healthy siblings (12\%) and healthy unrelated controls (13\%). Unable to detect a difference by feeding group in antibody responseto immunizations (except

Hemophilus influenza $b$ ), parent-reported diarrhea, or otitis media. No consistent differences were detected in immune status, maturation, or level of immunocompetence between soy and cow-milk formula-fed infants.

Improvement with discontinuation of soy formula or addition of iodine.

Oral thyroxine therapy did not decrease thyroidstimulating hormone until cow-milk formula was substituted for soy formula.

Switching from soy formula to cow-milk formula resulted in increased absorption of thyroid replacement doses.

Unable to detect a significant association overall between premature thelarche and soy infant formula intake; restriction of multivariate analysis to subjects with thelarche before age 2 years showed significant association (OR 2.7; 95\% CI 1.1-6.8).

Unable to detect infant feeding-related differences in adult height, weight, body-mass index, or sexual maturation history; duration of menstrual bleeding was 0.37 days longer and severe menstrual discomfort was more commonin women fed with soy formula than with cow-milk formula

There were no detectable group differences in height and weight, no signs of precocious puberty in girls, and no gynecomastia in boys.

Breast-fed infants had the highest serum cholesterol levels and lowest fractional cholesterol synthesis rate. There was an inverse relationship between cholesterol intake and fractional cholesterol synthesis.

Almost twice as many diabetic children had been soy formula-fedas had controls [not statistically significant; data were not shown].
Chandra and Hamed (1991); Chandra (1997, 1998);

Chandra et al.

(1989a,b)

Lack et al. (2003)

Zoppi et al. (1979)

Zoppi et al. (1982)

Zoppi et al. (1983)

May et al. (1982)

McDonald et al. (1984)

Fort et al. (1990)

Cordle et al. (2002); Ostrom et al. (2002)

Shepard et al. (1960)

Chorazy et al. (1995)

Jabbar et al. (1997)

Freni-Titulaer et al. (1986)

Strom et al. (2001)

Giampietro et al. (2004)

Cruz et al. (1994)

Fort et al. (1986) 
Children age 9-10 years who had been given soy formula without breast feeding $(\mathrm{n}=176)$ and who had received soy formula but also had had some breast feeding $(n=342)$.

Women age 25-64 years with newly-diagnosed breast cancer $(\mathrm{n}=1459)$ and age-matched women without breast cancer $(\mathrm{n}=$ 1556).

Adult Asian-American women with breast cancer $(\mathrm{n}=501)$

and age- and ethnicity-matched women without breast cancer (n =594).

Adult women with a mean age of $\sim 43$ who underwent mammography
Unable to detect an effect of breast feeding on IQ when controlled for parental education and family income.

Significant inverse association between recalled amount of dietary soy protein eaten during adolescence and breast cancer diagnosis.

Significant inverse association between recalled soy intake during adolescence and adult and breast cancer diagnosis.

Recall of the use of soy foods during the first 19 years of life was associated with more dense breast tissue and a slower decline in breast tissue density with age than recall of no soy food intake during those years.
Malloy and Berendes (1998)

Shu et al. (2001)

Wu et al. (2002)

Maskarinec et al. (2004b) 
Table 42

Developmental Effects of Soy Products in Experimental Animals ${ }^{a}$

\begin{tabular}{ll}
\hline Model & Experimental groups \\
\hline Long-Evans rat & $\begin{array}{l}\text { Soy milk }(\mathrm{n}=3) \text { or rice milk }(\mathrm{n}=2) \text { provided to dam } \\
\text { instead of water during lactation period }(\mathrm{n}=3) . \\
\end{array}$ \\
& Phytoestrogen-free AIN-93G feed was used.
\end{tabular}

Wistar derived rat

Long-Evans rat

Sprague-Dawley rat

Wistar rat

Sprague-Dawley rat

Han-NMRI mouse

Mouse

Marmoset
Dams ( $\mathrm{n}=12 /$ group) given assigned diet during pregnancy and lactation, and offspring weaned to assigned diet through PND 68. Diet assignments (dam/offspring) were:

RM3/RM1 (standard soy-based diets) AIN-76/AIN-76 (soy- and alfalfa-free diet)

RM3/AIN-76A

Global 2016/Global 2016 (soy- and alfalfa-free diet)

Purina 5001/Purina 5001 (based on soybeans and alfalfa)

Dams $(\mathrm{n}=5 /$ group) given phytoestrogen-containing diet $(600 \mu \mathrm{g} / \mathrm{g}$ diet $)$ or phytoestrogen-free fish mealbased diet throughout pregnancy and lactation. Offspring weaned to dam's diet.

Pregnant rats $(n=15 /$ goup $)$ given CRF-1, a soy-based diet, or NIH-07, a soy-free diet. On GD 15, seven dams/group also given ethinyl estradiol in the diet.

Dams given $15.5 \%$ soy-meal diet or soy-free diet before and during mating, pregnancy, and lactation ( $\mathrm{n}=7-29$, depending on endpoint).

Males ( $n=6-12 /$ group) from multi-generation study continued on 1 of 6 diets that had been given to their parents:

AIN-93G (soy-free, casein-based diet), Control diet + soy protein (isoflavones $31.7 \mathrm{mg} / \mathrm{kg}$ feed), or

Control diet + soy protein + isoflavone mixture at $36.1,74.5,235.8$, or $1046.6 \mathrm{mg} / \mathrm{kg}$ feed.

Pregnant dams fed $7 \%$ soybean meal diet or soy-free diet; half of male offspring were maintained on dam's diet and half were switched to opposite diet $(n=27$ 29/group)

Wild-type male mice fed soy-based or soy-free diet "from birth" ( $n=6-12 /$ group; study also included aromatase-knock out mice)

Twins fed from 4-5 days of age with soy infant formula or cow-milk formula during part of the day $(n=7-14$ twin pairs, depending on age of evaluation)

\section{Effects of soy product}

Reference

Female offspring

$\uparrow$ Body weight

$\downarrow$ Anogenital distance adjusted for body weight

$\downarrow$ Uterine progesterone receptor Male offspring

Male offspring

$\downarrow$ Relative epididymis weight

Dam exposure to RM3 diet

$\uparrow$ Testis and epididymis weights on PND 68

$\uparrow$ Birth and post-weaning weights

Offspring exposure to RM1 diet

Advanced puberty landmarks in male and female offspring

$\uparrow$ Uterine weight on PND 26 and 140-144

$\uparrow$ Male body weight on PND 128

$\uparrow$ Female body weight on PND 140-144

Delayed vaginal opening

$\downarrow$ Body weight on PND 50 in male and

female offspring

$\uparrow$ Male pup body weight on PND 2

$\uparrow$ Female pup body weight on PND 21

$\downarrow$ Female anogenital distance adjusted for body weight

Enhanced ethinyl estradiol effects on

female reproductive organ histopathology

$\uparrow$ Sertoli cell nuclear volume, PND 18

$\uparrow$ Spermatocyte/Sertoli cell nuclear

volume, PND 18 and 25

$\downarrow$ Body weight, PND 90-95

Testis weight, PND 90-95

$\uparrow$ Plasma FSH, PND 90-95

$\uparrow$ Testis weight, PND 28 in 3 highest isoflavone groups (total isoflavone intake $\geq 2 \mathrm{mg} / \mathrm{kg}$ bw/day)

$\uparrow$ Serum testosterone and

dihydrotestosterone, PND 120 in 2 highest

isoflavone groups (total isoflavone intake

$\geq 6.3 \mathrm{mg} / \mathrm{kg}$ bw/day)

$\uparrow$ Testicular $3 \beta$-hydroxysteroid

dehydrogenase activity, PND 28, in 2

highest isoflavone groups

[Based on PND 28 testis weight:

NOAEL $1.2 \mathrm{mg} / \mathrm{kg}$ bw/day and LOAEL

$2.0 \mathrm{mg} / \mathrm{kg} \mathrm{bw} / \mathrm{day}]$

Effects of offspring exposure

$\uparrow$ Prostate weight in 2-month-old

offspring

$\uparrow$ Relative male reproductive organ

weight at 1 year

14 weeks of age

$\uparrow$ Seminiferous tubule diameter

$\downarrow$ Seminiferous tubule length

$\downarrow$ Sertoli cell numbers 1 year of age

$\downarrow$ Testicular epithelial volume

$\uparrow$ Testicular interstitial volume

$\downarrow$ Seminiferous tubule length

$\downarrow$ Plasma testosterone PND 35-45

$\uparrow$ Testis weight, Sertoli cell and Leydig cell numbers at 120-138 weeks of age
Lund et al.

(2001a)

Hughes et al.

(2004)

Odum et al.

(2001)

Masutomi et

al. (2004)

Atanassova et al. (2000)

McVey et al. (2004a,b)

Mäkelä et al. (1995b)

Robertson et al. (2002)

Sharpe et al. (2002); Tan et al. (2006) 


\begin{tabular}{llll}
\hline Model & Experimental groups & Effects of soy product & Reference \\
\hline Pig & $\begin{array}{l}\text { Piglets }(\mathrm{n}=16 \text { from 4 litters) randomly assigned 48\% } \\
\text { soybean meal-based liquid diet or cow-milk based } \\
\text { diet; protein source continued after weaning until 56 } \\
\text { days of age. }\end{array}$ & $\begin{array}{l}\downarrow \text { Body weight gain at 28 days of age } \\
\text { Intestinal villi shortened at 28 but not 56 } \\
\text { days of age }\end{array}$ & $\begin{array}{l}\text { Li et al. } \\
(1990)\end{array}$ \\
\hline
\end{tabular}

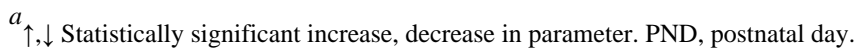


Table 43

Statistically Significant Hormonal Effects in Women Fed Textured Vegetable Protein Containing 45 mg/Day Conjugated Isoflavone ${ }^{a}$

\begin{tabular}{llrl}
\hline Parameter & Control diet & Isoflavone-enriched diet \\
\hline Follicular phase length, days & $15.0 \pm 0.4$ & $17.5 \pm 0.9$ & {$[\uparrow 17 \%]$} \\
Plasma 17ß-estradiol, follicular phase, $\mathrm{nM}$ & $0.25 \pm 0.03$ & $0.35 \pm 0.1$ & $5.8 \pm 1.6$ \\
Plasma LH, mid-cycle, IU/mL & $22.4 \pm 5.3$ & $8.9 \pm 1.8$ & {$[\uparrow 40 \%]$} \\
Plasma FSH, mid-cycle, IU/mL & $15.5 \pm 2.4$ & {$[\downarrow 74 \%]$} & {$[\downarrow 43 \%]$} \\
\hline
\end{tabular}

${ }^{a}$ From Cassidy et al. (1995). Values are presented as mean \pm SEM. Results presented as mean \pm SD are available in Cassidy et al. (1994), which gives slightly different results for some parameters. 
Table 44

Effects of Soy Milk Consumption on Menstrual Cycle Length and Hormones ${ }^{a}$

\begin{tabular}{|c|c|c|c|}
\hline \multirow[b]{2}{*}{ Parameter } & \multicolumn{3}{|c|}{ Months after soy milk consumption phase } \\
\hline & 0 (During) & 1 & $2-3$ \\
\hline Menstrual cycle length & $" \uparrow "(P=0.06)$ & $" \uparrow "(P=0.11)$ & $\leftrightarrow$ \\
\hline \multicolumn{4}{|l|}{ Serum $17 \beta$-estradiol } \\
\hline Cycle average & $\downarrow$ & $\downarrow$ & $" \downarrow " P=0.11$ \\
\hline \multicolumn{4}{|l|}{ Cycle days } \\
\hline $5-7$ & $" \downarrow "(P=0.09)$ & $\leftrightarrow$ & $\leftrightarrow$ \\
\hline $12-14$ & $\downarrow \downarrow \downarrow$ & $" \downarrow "(P=0.056)$ & $\downarrow$ \\
\hline $20-22$ & $\downarrow$ & $\downarrow \backslash \downarrow$ & $" \downarrow "(P=0.24)$ \\
\hline Serum progesterone, luteal phase & $\downarrow$ & $\leftrightarrow$ & $\leftrightarrow$ \\
\hline $\begin{array}{l}\text { Serum dehydroepiandrosterone sulfate, } \\
\text { cycle average }\end{array}$ & $\downarrow$ & $\leftrightarrow$ & $\uparrow^{\prime \prime}(P=0.08)$ \\
\hline
\end{tabular}

$a_{\mathrm{n}=4-6}$ subjects per time period. $\uparrow, \downarrow, \leftrightarrow$ increase, decrease, or no change compared to baseline value at $\mathrm{P} \leq 0.05$ by paired $t$-test. " $\uparrow, " ~ " \downarrow "$ increase or decrease described by study authors with $P>0.05$.

From Lu et al. (1996). 


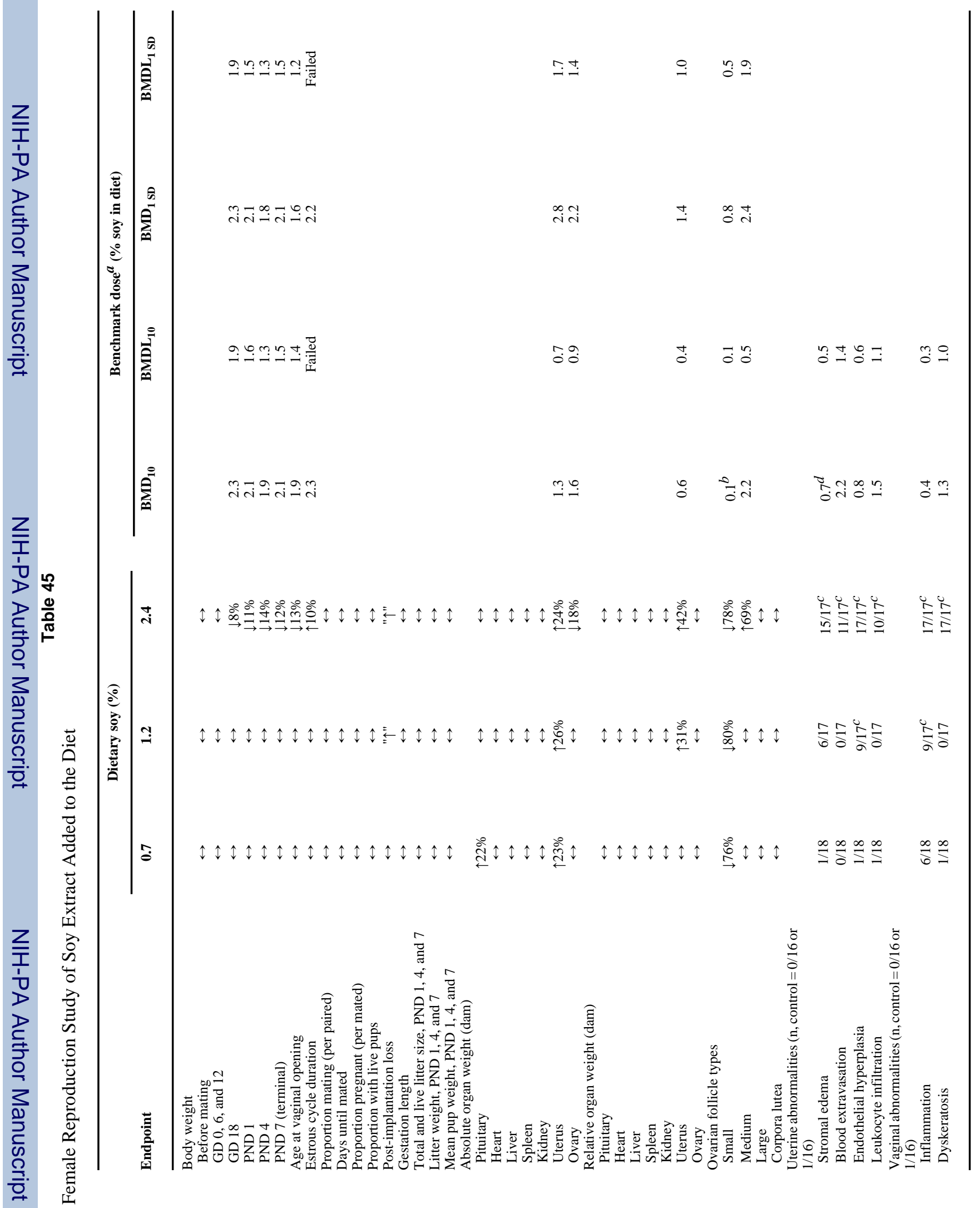

Birth Defects Res B Dev Reprod Toxicol. Author manuscript; available in PMC 2008 March 11. 


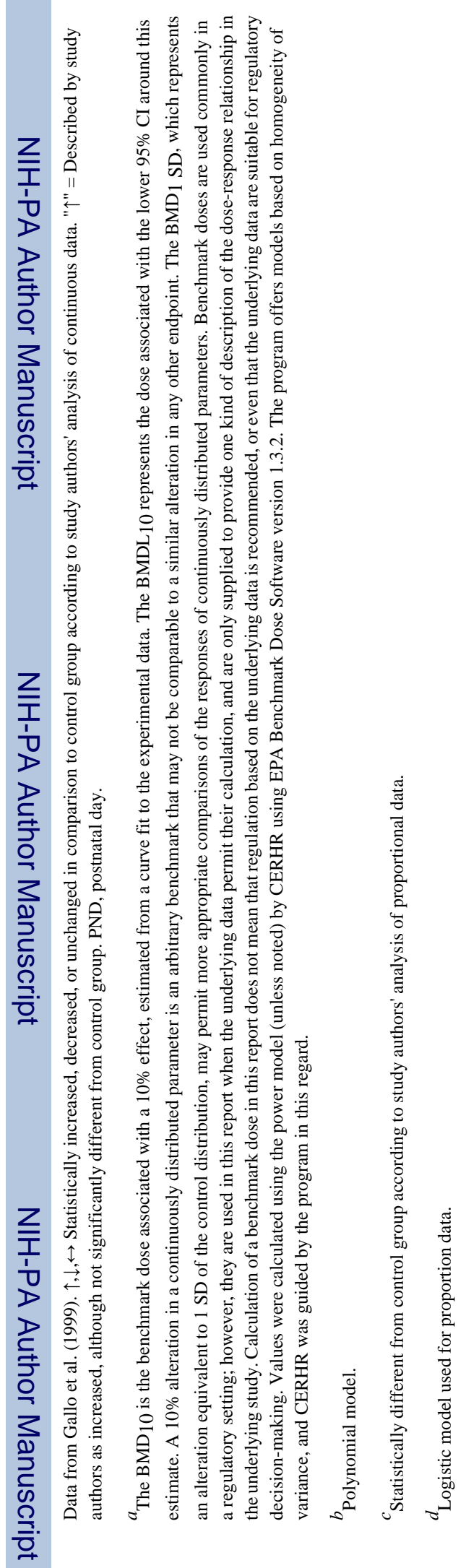

Birth Defects Res B Dev Reprod Toxicol. Author manuscript; available in PMC 2008 March 11. 
Table 46

Effects on Adult Male Rats of Feeding Soy-Based Diet ${ }^{a}$

\begin{tabular}{lc}
\hline Parameter & Comparison to phytoestrogen-free diet \\
\hline Feed intake & $\leftrightarrow$ \\
Water intake & $\uparrow \%$ \\
Body weight & $\downarrow 5 \%$ \\
Prostate weight, absolute & $\downarrow 7 \%$ \\
Relative to body weight & $\downarrow \%$ \\
Plasma hormone concentration & $\downarrow 50 \%$ \\
Testosterone & $\leftrightarrow$ \\
LH & $\downarrow 85 \%$ \\
Androstenedione & $\leftrightarrow$ \\
Estradiol & $\leftrightarrow$ \\
Prostatic $5 \alpha-$ reductase activity & $\leftrightarrow$ \\
Testicular steroidogenic acute regulatory protein & $\leftrightarrow$ \\
\end{tabular}

${ }^{a}$ From Weber et al. (2001b). $\uparrow, \downarrow, \leftrightarrow$ Statistically significant increase, decrease, or no change comparing male rats fed a soy-based diet containing total phytoestrogens $600 \mu \mathrm{g} / \mathrm{g}$ feed with male rats fed a phytoestrogen-free diet. 
Table 47

Summary of Reproductive Studies in Women ${ }^{a}$

\begin{tabular}{l} 
Intervention, $\mathbf{n}=$ starting sample \\
\hline Diet for 1 month containing $1 \mathrm{of}$ : \\
• Textured vegetable protein $60 \mathrm{~g} /$ \\
day \\
- Textured vegetable protein $28 \mathrm{~g} /$ \\
day \\
- Miso $50 \mathrm{~g} /$ day \\
• Isoflavone-free soy product, 60 \\
g/day $\mathrm{n}=3-6 /$ group \\
Soy milk 36 ounces/day for 1 \\
month, $\mathrm{n}=6$
\end{tabular}

No intervention: Women were questioned about dietary intakes, $\mathrm{n}$ $=50$

Soy milk, $400 \mathrm{~mL} /$ day $(109 \mathrm{mg} /$ day isoflavones) for 2 months spanning parts of 3 menstrual cycles, $n=31$

Commercial soy supplement providing total isoflavones 0.16 , 1.01 , or $2.01 \mathrm{mg} / \mathrm{kg}$ bw/day, $\mathrm{n}=12$

Soy supplement containing $38 \mathrm{mg}$ total isoflavones given daily for 2 menstrual cycles, $\mathrm{n}=16$ regularly cycling women and 20 women on oral contraceptives

Soy supplement resulting in daily isoflavone intake of $0.15,1.01$, or $2.01 \mathrm{mg} / \mathrm{kg}$ bw/day, taken for 3 menstrual cycles plus 9 days, followed by 3 -week washout, $n=14$

Isoflavone supplement tablet providing 20 or $40 \mathrm{mg} /$ day for 1 menstrual cycle, $n=19$ or 20 for both groups combined Soy milk 36 ounces/day for 1 month, $\mathrm{n}=10$

Reduced-isoflavone soy supplement, $\mathrm{n}=9$ for 1 cycle

No intervention: Women were questioned about dietary intakes, $\mathrm{n}$ $=200$

\author{
Control \\ Women received control diet for 1 \\ month and served as their own \\ controls.
}

Blood samples were taken before soy milk, during soy-milk consumption, and for a variable number of cycles afterwards, with comparisons made between soy and non-soy time periods.

No control group. Correlation testing was used for analysis of serum $17 \beta$ estradiol and sex hormone-binding globulin levels and soy intake. Adjustments were made for age, body-mass index, cycle length, and energy, fat, and fiber intake.

Women who were instructed not to consume soy milk, $\mathrm{n}=29$.

Cross-over design with women serving as their own controls

Cross-over design using soy-free milk treatment; women served as their own controls.

Cross-over study with each woman serving as her own control; the 0.15 $\mathrm{mg} / \mathrm{kg}$ bw/day isoflavone intake was the control condition.

$\mathrm{n}=20$; unknown whether a placebo tablet was taken.

Women were monitored during a 3month baseline period and served as their own controls.

Women were monitored for a baseline cycle before treatment and served as their own controls.

No control group. Correlation testing was used for analysis of serum $17 \beta$ estradiol, estrone, estriol, LH, and

\begin{tabular}{ll} 
Effects of soy product & Reference \\
\hline $\begin{array}{l}\text { Textured vegetable protein } 60 \mathrm{~g} / \text { day }(45 \\
\text { mg/day conjugated isoflavones): }\end{array}$ & $\begin{array}{l}\text { Cassidy et al. } \\
\uparrow 17 \% \text { in follicular-phase length }\end{array}$ \\
$\uparrow 40 \%$ in follicular-phase plasma $17 \beta-$ & \\
estradiol \\
$\downarrow 74 \%$ in mid-cycle plasma LH \\
$\downarrow 43 \%$ in mid-cycle plasma FSH
\end{tabular}

$\uparrow$ Menstrual cycle length $(P=0.06)$

$\downarrow$ Serum $17 \beta$-estradiol (throughout cycle)

$\downarrow$ Luteal-phase serum progesterone

$\downarrow$ Serum dehydroepiandrosterone sulfate (cycle average)

Negative association between soy intake and serum $17 \beta$-estradiol on cycle Day 22 . Negative association between serum sex hormone-binding globulin and miso consumption on cycle Day 22.

$\downarrow 23-30 \%$ in mid-cycle serum estrone in soy-milk group Menstrual cycle lengthened over soy-milk treatment interval.

Urinary isoflavones and lignans

increased and endogenous estrogens and their metabolites decreased with increasing supplement dose.

Unable to detect effects of soy supplementation on menstrual cycle length or on serum 17 $\beta$-estradiol, estrone, dehydroepiandrosterone sulfate, prolactin, or sex hormone-binding globulin; no detectable effect on urinary levels of estrogen metabolites

Unable to detect an effect of soy supplement on follicular phase, luteal phase, or total cycle length.

$\downarrow$ Mid-cycle plasma estrone (high versus low dose).

$\downarrow$ Periovulatory plasma LH and FSH (low dose versus control)

Unable to detect a change in plasma $17 \beta-$ estradiol or progesterone.

$\uparrow$ Plasma 17ß-estradiol

[Many cycles were irregular and the Methods and Results were confusing.] $\downarrow$ Luteal phase length $(P=0.07)$, positively associated with urinary isoflavone excretion and with decreased protein intake.

$\downarrow$ Serum $17 \beta$-estradiol (AUC), positively associated with plasma and urinary isoflavones and inversely related to protein intake.

Unable to detect an effect on serum LH or FSH.

$\downarrow$ Serum $17 \beta$-estradiol during each phase of the cycle and overall; significance lost when corrections were made for macronutrient intake.

$\downarrow$ Serum progesterone; significance lost when corrected for protein intake.

Unable to detect a change in serum LH, $\mathrm{FSH}$, or sex hormone-binding globulin. Estrone correlated with intake of soy protein

Soy-protein intake higher than the median for the sample associated with
Lu et al. (1996)

Nagata et al. (1997)

Nagata et al. (1998)

Xu et al.

(1998)

Martini et al. (1999)

Duncan et al. (1999a)

Watanabe et al. (2000)

Lu et al.

(2000a)

Lu et al. (2001)

Jakes et al. (2001) 


\begin{tabular}{|c|c|c|c|}
\hline Intervention, $n=$ starting sample & Control & Effects of soy product & Reference \\
\hline $\begin{array}{l}\text { Tofu, soy milk, and soybeans with } \\
\text { a mean } \pm \text { SD isoflavone intake of } \\
32.0 \pm 10.5 \mathrm{mg} / \mathrm{day}, \mathrm{n}=20\end{array}$ & $\begin{array}{l}\text { FSH and for analysis of menstrual } \\
\text { cycle parameters from diaries. } \\
\text { Women were monitored for two } \\
\text { baseline cycles before treatment and } \\
\text { served as their own controls. }\end{array}$ & $\begin{array}{l}\text { longer cycle length }(P=0.06 \text { adjusted for } \\
\text { age and parity). } \\
\downarrow \text { Luteal phase serum } 17 \beta \text {-estradiol. } \\
\uparrow \text { Serum sex hormone-binding globulin in } \\
\text { non-Asian subjects. Unable to detect an } \\
\text { effect on menstrual cycle length or length } \\
\text { of follicular or luteal phases. }\end{array}$ & $\begin{array}{l}\text { Wu et al. } \\
(2000)\end{array}$ \\
\hline $\begin{array}{l}\text { Two servings/day of soy foods that } \\
\text { could include tofu, soy milk, } \\
\text { roasted soy nuts, soy bars, or soy } \\
\text { protein powder ( } 1 \text { serving } \approx 25 \mathrm{mg} \\
\text { isoflavones), } \mathrm{n}=109\end{array}$ & $\begin{array}{l}\text { Age and ethnicity-matched women } \\
\text { maintaining their usual diet, } \mathrm{n}=111 \text {. }\end{array}$ & $\begin{array}{l}\text { Unable to detect an effect on menstrual } \\
\text { cycle length or serum concentration of } \\
17 \beta \text {-estradiol, estrone, androstenedione, } \\
\text { progesterone, or sex hormone-binding } \\
\text { globulin. }\end{array}$ & $\begin{array}{l}\text { Maskarinec et } \\
\text { al. }(2004 a)\end{array}$ \\
\hline
\end{tabular}

$a_{\downarrow, \uparrow \text { Significant decrease, increase. }}$ 
Table 48

Summary of Reproductive Studies in $\mathrm{Men}^{a}$

\begin{tabular}{|c|c|c|c|}
\hline $\begin{array}{l}\text { Intervention, } \mathrm{n}=\text { starting } \\
\text { sample }\end{array}$ & Control & Effects of soy product & Reference \\
\hline $\begin{array}{l}\text { Dietary tofu } 290 \mathrm{~g} / \text { day }(35 \mathrm{~g} / \\
\text { day soy protein) for } 1 \text { month, } \mathrm{n} \\
=42\end{array}$ & $\begin{array}{l}\text { Dietary meat } 150 \mathrm{~g} / \text { day for } 1 \text { month; cross-over } \\
\text { design with each man serving as his own control. }\end{array}$ & $\begin{array}{l}\text { Unable to detect an effect on serum } \\
\text { total testosterone, } \\
\text { dihydrotestosterone, } \\
\text { androstanediol glucuronide, or } \\
17 \beta \text {-estradiol. } \\
\downarrow 12 \% \text { in weight-adjusted } \\
\text { testosterone:estradiol ratio. } \\
\uparrow 9 \% \text { in weight adjusted sex } \\
\text { hormone-binding globulin. }\end{array}$ & $\begin{array}{l}\text { Habito et al. } \\
(2000)\end{array}$ \\
\hline $\begin{array}{l}\text { No intervention: Men were } \\
\text { questioned about dietary } \\
\text { intakes, } \mathrm{n}=97\end{array}$ & $\begin{array}{l}\text { No control group. Correlation analysis was used } \\
\text { for analysis of serum } 17 \beta \text {-estradiol, estrone, total } \\
\text { and free testosterone, dihydrotestosterone, and } \\
\text { sex hormone-binding globulin with adjustment } \\
\text { for age, body-mass index, smoking, and ethanol } \\
\text { use, and (for androgens) dietary fat, cholesterol, } \\
\text { carbohydrate, and vitamins } \mathrm{B}_{1} \text { and D. }\end{array}$ & $\begin{array}{l}\text { Serum } 17 \beta \text {-estradiol inversely } \\
\text { correlated with intake of soy } \\
\text { products. } \\
\text { Unable to detect other significant } \\
\text { correlations. }\end{array}$ & $\begin{array}{l}\text { Nagata et al. } \\
(2000)\end{array}$ \\
\hline $\begin{array}{l}\text { Soy supplement ( } 40 \mathrm{mg} \text { total } \\
\text { isoflavones) daily for } 2 \\
\text { months, } \mathrm{n}=15\end{array}$ & $\begin{array}{l}\text { Men were evaluated before, during, and after the } \\
\text { supplementation period and served as their own } \\
\text { controls. }\end{array}$ & $\begin{array}{l}\text { Unable to detect an effect on } \\
\text { testicular volume, semen analysis } \\
\text { parameters, or plasma levels of } \\
17 \beta \text {-estradiol, testosterone, FSH, or } \\
\text { LH. }\end{array}$ & $\begin{array}{l}\text { Mitchell et al. } \\
(2001)\end{array}$ \\
\hline $\begin{array}{l}\text { Soy milk } 400 \mathrm{~mL} / \text { day for } 8 \\
\text { weeks, } \mathrm{n}=17\end{array}$ & Regular diet without soy milk, $\mathrm{n}=17$. & $\begin{array}{l}\downarrow \text { Serum estrone. } \\
\text { Unable to detect changes in serum } \\
17 \beta \text {-estradiol, total and free } \\
\text { testosterone, or sex hormone- } \\
\text { binding globulin. }\end{array}$ & $\begin{array}{l}\text { Nagata et al. } \\
(2001)\end{array}$ \\
\hline
\end{tabular}

$a_{\downarrow, \uparrow \text { Significant decrease, increase. }}$ 
Table 49

Estimated Total Isoflavone Intake in Infants Fed Soy Formula

\begin{tabular}{ll}
\hline Country & Total Isoflavone intake $\mathbf{~ g / / k g ~ b w / d a y ~}$ \\
\hline U.S. & $\sim 1.6-12$ \\
U.K. & $1.7-5$ \\
New Zealand & $2.9-3.8$ \\
\hline
\end{tabular}

